\title{
Non-Uniform Constellations for Next-Generation Digital Terrestrial Broadcast Systems
}

Departamento de Comunicaciones

Universitat Politècnica de València

A thesis for the degree of

PhD in Telecommunications Engineering

Valencia, June 2017

Author:

Manuel Fuentes Muela

Supervisors:

Dr. David Gómez Barquero Prof. Narcís Cardona Marcet 



\section{Abstract}

Nowadays, the digital terrestrial television (DTT) market is characterized by the high capacity needed for high definition TV services, and the limited spectrum available. There is a need for an efficient use of the broadcast spectrum, which requires new technologies to guarantee increased capacities. NonUniform Constellations (NUC) arise as one of the most innovative techniques to approach those requirements. These constellations have been implemented in next-generation broadcast systems such as DVB-NGH (Digital Video Broadcasting - Next Generation Handheld) or ATSC 3.0 (Advanced Television Systems Committee - Third Generation). NUCs reduce the gap between uniform Gray-labelled Quadrature Amplitude Modulation (QAM) constellations and the theoretical unconstrained Shannon limit. With these constellations, symbols are optimized in both in-phase (I) and quadrature (Q) components by means of signal geometrical shaping, considering a certain signal-to-noise ratio (SNR) and channel model.

There are two types of NUC, depending on the number of real-valued dimensions considered in the optimization process, i.e. one-dimensional and two dimensional NUCs (1D-NUC and 2D-NUC, respectively). 1D-NUCs maintain the squared shape from QAM, but relaxing the distribution between constellation symbols in a single component, with non-uniform distance between them. These constellations provide better SNR performance than QAM, without any demapping complexity increase. 2D-NUCs also relax the square shape constraint, allowing to optimize the symbol positions in both dimensions, thus achieving higher capacity gains and lower SNR requirements. However, the use of 2D-NUCs implies a higher demapping complexity, since a 2D-demapper is needed, i.e. I and Q components cannot be separated.

In this dissertation, NUCs are analyzed from both transmit and receive point of views, using either single-input single-output (SISO) or multiple-input multiple-output (MIMO) antenna configurations. In SISO transmissions, 1DNUCs and 2D-NUCs are optimized for a wide range of SNRs, several channel models and different constellation orders, using the Nelder-Mead optimization 


\section{ABSTRACT}

algorithm. The optimization of rotated 2D-NUCs is also investigated, including the rotation angle as an additional variable in the optimization. Even though the demapping complexity is not increased, the SNR gain of these constellations is not significant. The highest rotation gain is obtained for low-order constellations and high SNRs. However, with multi-RF techniques such as Channel Bonding (CB) or Time-Frequency Slicing (TFS), the SNR gain is drastically increased, since I and Q components are transmitted in different RF channels. In this thesis, multi-RF gains of NUCs with and without rotation are provided for some representative scenarios.

At the receiver, two different implementation bottlenecks are explored. First, the demapping complexity of all considered constellations is analyzed. Afterwards, two complexity reduction algorithms for 2D-NUCs are proposed. Both algorithms drastically reduce the number of distances to compute the output log-likelihood ratios (LLR). Moreover, both are finally combined in a single demapper. Quantization of NUCs is also explored in this dissertation, since LLR values and I/Q components are modified when using these constellations, compared to traditional QAM constellations. A new algorithm that is based on the optimization of the quantizer levels for a particular constellation is proposed. The proposed algorithm reduces the number of quantization bits and can be also extrapolated to QAM.

The use of NUCs in multi-antenna communications is also investigated. In this dissertation, parameters that affect the optimization process are evaluated, when using a $2 \times 2$ dual polarized MIMO system. It includes the optimization in one or two antennas, the use of power imbalance, the cross-polar discrimination (XPD) between receive antennas, the use of different optimum and sub-optimum demappers, equalization methods and different channel models. Assuming different values for the parameters evaluated, new Multi-Antenna Non-Uniform Constellations (MA-NUC) are obtained by means of a particularized re-optimization process, specific for MIMO. At the receiver, an extended demapping complexity analysis is performed, where it is shown that the use of 2D-NUCs in MIMO extremely increases the demapping complexity. In multiantenna systems, the optimum demapping complexity grows exponentially with the number of antennas and the constellation order. As an alternative, an efficient solution for 2D-NUCs and MIMO systems based on Soft-Fixed Sphere Decoding (SFSD) is proposed. The main drawback is that SFSD demappers do not work with 2D-NUCs, since they perform a Successive Interference Cancellation (SIC) step that needs to be performed in separated I and Q components. The proposed method quantifies the closest symbol using Voronoi regions and allows SFSD demappers to work. 


\section{Resumen}

Hoy en día, el mercado de la televisión digital terrestre (TDT) está caracterizado por la alta capacidad requerida para transmitir servicios de televisión de alta definición y el espectro disponible, el cual se encuentra muy limitado. Es necesario por tanto un uso eficiente del espectro radioeléctrico, el cual requiere nuevas tecnologías para garantizar mayores capacidades. Las constelaciones no-uniformes (NUC) emergen como una de las técnicas más innovadoras para abordar tales requerimientos. Estas constelaciones han sido adoptadas en sistemas de televisión de siguiente generación tales como DVB-NGH (Digital Video Broadcasting - Next Generation Handheld) o ATSC 3.0 (Advanced Television Systems Committee - Third Generation). Las NUC reducen el espacio existente entre las constelaciones uniformes QAM y el límite teórico de Shannon. Con estas constelaciones, los símbolos se optimizan en ambas componentes fase (I) y cuadratura (Q) mediante técnicas geométricas de modelado de la señal, considerando un nivel señal a ruido (SNR) concreto y un modelo de canal específico.

Hay dos tipos de NUC, dependiendo del número de dimensiones reales consideradas en el proceso de optimización, es decir, NUCs unidimensionales y bidimensionales (1D-NUC y 2D-NUC, respectivamente). Las 1D-NUC mantienen la forma cuadrada de las QAM, pero permiten cambiar la distribución entre los símbolos en una componente concreta, teniendo una distancia no uniforme entre ellos. Estas constelaciones proporcionan un mejor rendimiento SNR que QAM, sin ningún incremento en la complejidad en el demapper. Las 2D-NUC también permiten cambiar la forma cuadrada de la constelación, permitiendo optimizar los símbolos en ambas dimensiones y por tanto obteniendo mayores ganancias en capacidad y menores requerimientos en SNR. Sin embargo, el uso de 2D-NUCs implica una mayor complejidad en el receptor, puesto que se necesita un demapper 2D, donde las componentes I y Q no pueden ser separadas.

En esta tesis se analizan las NUC desde el punto de vista tanto de transmisión como de recepción, utilizando bien configuraciones con una antena (SISO) o con múltiples antenas (MIMO). En transmisiones SISO, se han op- 


\section{RESUMEN}

timizado 1D-NUCs para un rango amplio de distintas SNR, distintos modelos de canal y varios órdenes de constelación. También se ha investigado la optimización de 2D-NUCs rotadas, donde el ángulo de rotación se incluye en la optimización como una variable adicional. Aunque la complejidad no aumenta, la ganancia SNR de estas constelaciones no es significativa. La mayor ganancia por rotación se obtiene para bajos órdenes de constelación y altas SNR. Sin embargo, utilizando técnicas multi-RF como Channel Bonding (CB) o TimeFrequency Slicing (TFS), la ganancia aumenta drásticamente puesto que las componentes I y Q se transmiten en distintos canales RF. En esta tesis, se han estudiado varias ganancias multi-RF representativas de las NUC, con o sin rotación.

En el receptor, se han identificado dos cuellos de botella diferentes en la implementación. Primero, se ha analizado la complejidad en el receptor para todas las constelaciones consideradas y, posteriormente, se proponen dos algoritmos para reducir la complejidad con 2D-NUCs. Ambos algoritmos reducen drásticamente el número de distancias para computar los LLR en el demapper con 2D-NUCs. Además, los dos pueden combinarse en un único demapper. También se ha explorado la cuantización de estas constelaciones, ya que tanto los valores LLR como las componentes I/Q se ven modificados, comparando con constelaciones QAM tradicionales. Además, se ha propuesto un algoritmo que se basa en la optimización para diferentes niveles de cuantización, para una NUC concreta. El algoritmo propuesto reduce el número de bits a utilizar y puede ser utilizado también con QAM.

Igualmente, se ha investigado en detalle el uso de NUCs en MIMO. En esta tesis se han evaluado los distintos parámetros que afectan al proceso de optimización cuando se utilizan sistemas MIMO $2 \times 2$ dual polarizados. Se ha incluido la optimización en una sola o en dos antenas, el uso de un desbalance de potencia, factores de discriminación entre antenas receptoras (XPD), el uso de distintos demappers óptimos y subóptimos, métodos de ecualización y distintos canales. Asumiendo distintos valores, se han obtenido nuevas constelaciones multi-antena (MA-NUC) gracias a un nuevo proceso de re-optimización específico para MIMO. En el receptor, se ha extendido el análisis de complejidad en el demapper, la cual se incrementa enormemente con el uso de 2D-NUCs y sistemas MIMO. En concreto, la complejidad aumenta exponencialmente con el número de antenas y el orden de constelación. Como alternativa, se propone una solución basada en el algoritmo Soft-Fixed Sphere Decoding (SFSD). El principal problema es que estos demappers no funcionan con 2D-NUCs, puesto que necesitan de un paso adicional en el que las componentes I y Q necesitan separarse. El método propuesto cuantifica el símbolo más cercano utilizando las regiones de Voronoi, permitiendo el uso de este tipo de receptor. 


\section{Resum}

Actualment, el mercat de la televisió digital terrestre (TDT) està caracteritzat per l'alta capacitat requerida per a transmetre servicis de televisió d'alta definició i l'espectre disponible, el qual es troba molt limitat. És necessari per tant un ús eficient de l'espectre radioelèctric, el qual requereix noves tecnologies per a garantir majors capacitats i millors servicis. Les constel-lacions no-uniformes (NUC) emergeixen com una de les tècniques més innovadores en els sistemes de televisió de següent generació per a abordar tals requeriments. Les NUC redueixen l'espai existent entre les constel-lacions uniformes QAM i el límit teòric de Shannon. Amb estes constel-lacions, els símbols s'optimitzen en ambdós components fase (I) i quadratura (Q) per mitjà de tècniques geomètriques de modelatge del senyal, considerant un nivell senyal a soroll (SNR) concret i un model de canal específic.

Hi ha dos tipus de NUC, depenent del nombre de dimensions reals considerades en el procés d'optimització, és a dir, NUCs unidimensionals i bidimensionals (1D-NUC i 2D-NUC, respectivament). 1D-NUCs mantenen la forma quadrada de les QAM, però permet canviar la distribució entre els símbols en una component concreta, tenint una distància no uniforme entre ells. Estes constel-lacions proporcionen un millor rendiment SNR que QAM, sense cap increment en la complexitat al demapper. 2D-NUC també canvien la forma quadrada de la constel-lació, permetent optimitzar els símbols en ambdós dimensions i per tant obtenint majors guanys en capacitat i menors requeriments en SNR. No obstant això, l'ús de 2D-NUCs implica una major complexitat en el receptor, ja que es necessita un demapper $2 \mathrm{D}$, on les components I i $\mathrm{Q}$ no poden ser separades.

En esta tesi s'analitzen les NUC des del punt de vista tant de transmissió com de recepció, utilitzant bé configuracions amb una antena (SISO) o amb múltiples antenes (MIMO). En transmissions SISO, s'han optimitzat 1D-NUCs, per a un rang ampli de distintes SNR, diversos models de canal i diferents ordes de constel-lació. També s'ha investigat l'optimització de 2D-NUCs rotades, on l'angle de rotació s'inclou en l'optimització com una variable addicional. 
Encara que la complexitat no augmenta, el guany SNR d'estes constel-lacions no és significativa. El major guany per rotació s'obté per a baixos ordes de constel-lació i altes SNR. No obstant això, utilitzant tècniques multi-RF com Channel Bonding (CB) o Time-Frequency Slicing (TFS), el guany augmenta dràsticament ja que les components I i Q es transmeten en distints canals RF. En esta tesi, s'ha estudiat el guany multi-RF de les NUC, amb o sense rotació.

En el receptor, s'han identificat dos colls de botella diferents en la implementació. Primer, s'ha analitzat la complexitat en el receptor per a totes les constel-lacions considerades i, posteriorment, es proposen dos algoritmes per a reduir la complexitat amb 2D-NUCs. Ambdós algoritmes redueixen dràsticament el nombre de distàncies per a computar els LLR en el demapper amb 2D-NUCs. A més, els dos poden combinar-se en un únic demapper. També s'ha explorat la quantització d'estes constel-lacions, ja que tant els valors LLR com les components I/Q es veuen modificats, comparant amb constel-lacions QAM tradicionals. A més, s'ha proposat un algoritme que es basa en l'optimització per a diferents nivells de quantització, per a una NUC concreta. L'algoritme proposat redueix el nombre de bits a utilitzar i pot ser utilitzat també amb QAM.

Igualment, s'ha investigat en detall l'ús de NUCs en MIMO. En esta tesi s'han avaluat els distints paràmetres que afecten el procés d'optimització quan s'utilitzen sistemes $2 \times 2$ MIMO dual polaritzats. S'ha inclòs l'optimització en una sola o en dos antenes, l'ús d'un desbalanç de potència, factors de discriminació entre antenes receptores (XPD), l'ús de distints demappers òptims i subòptims, mètodes d'equalització i distints canals. Assumint distints valors, s'han obtingut noves constel-lacions multi-antena (MA-NUC) gràcies a un nou procés de re-optimització específic per a MIMO. En el receptor, s'ha modificat l'anàlisi de complexitat al demapper, la qual s'incrementa enormement amb l'ús de 2D-NUCs i sistemes MIMO. En concret, la complexitat augmenta exponencialment amb el nombre d'antenes i l'orde de constel-lació. Com a alternativa, es proposa una solució basada en l'algoritme Soft-Fixed Sphere Decoding (SFSD) . El principal problema és que estos demappers no funcionen amb 2D-NUCs, ja que necessiten d'un pas addicional en què les components I i Q necessiten separar-se. El mètode proposat quantifica el símbol més pròxim utilitzant les regions de Voronoi, permetent l'ús d'este tipus de receptor. 


\section{Acknowledgements}

First of all, I would like to thank my two supervisors for their assistance and guidance. From the very beginning, Dr. David Gómez Barquero gave me the opportunity to be part of the Institute de Telecommunications and Multimedia Applications (iTEAM) at the Universitat Politècnica de València (UPV). His advice and support during all these years has been fundamental to improve as an engineer and researcher. Prof. Narcís Cardona also received me with open arms and gave me the chance to pursuit my Ph.D. in his group. Thanks to both of them, I could present my work to many people around the world, enjoying the experience and achieving new skills.

Very special thanks go to my colleagues of the Mobile Communications Group (MCG). During these three years, the people that had been part of this group have helped me to grow not only as a professional, but also as a person. Thank you to my friends Gerardo, Edu, Carlos Andreu, Carlos Barjau, Alejandro, Jordi Joan, Shitomi, Conchi, Tere, Josetxo, Carlos Herranz, Alicia, Sonia, Irene, Jorge, Sofía, Martina, José Luis, Sergio, Sandra and many others. I also want to thank my old colleagues David Vargas, Jaime and Jefferson for the good moments we spent together. I'm also indebted to Prof. Gerald Matz from the Technical University of Vienna, for inviting me to his research team. It has been one of the best experiences in my life, and I will be eternally grateful to him. Very special thanks also go to Georg Pichler, who helped me every single day during my stay there.

$Y$ por supuesto, esta tesis va dedicada a toda mi familia. En especial a mis padres, por darme la oportunidad de venir a Valencia a estudiar y convertirme en lo que hoy en día soy. A mi hermano, por los grandes momentos que hemos vividos juntos y los que nos quedan por vivir. Y a mi novia, por su grandísimo apoyo, amor incondicional e incontables consejos que me ha dado durante estos años. Os quiero a todos. 


\section{Table of contents}

1 Introduction $\quad 15$

1.1 Evolution and New Challenges of Digital Terrestrial Broadcasting 15

1.2 Preliminaries . . . . . . . . . . . . . . . . . . . 19

1.3 Research Challenges on Non-Uniform Constellations . . . . . . 22

1.4 Objectives and Scope . . . . . . . . . . . . . . . . . 23

1.5 State-of-the-Art . . . . . . . . . . . . . . . . 25

1.6 Thesis Outline and Contributions . . . . . . . . . . . . 31

1.7 List of Publications . . . . . . . . . . . . . . . . . . 34

1.7.1 Publications and Activities Related to this Thesis ... 34

1.7.2 Other Publications . . . . . . . . . . . . 35

2 Background 37

2.1 System Model Overview . . . . . . . . . . . . . . . . . 37

2.1.1 Multi-Antenna Considerations . . . . . . . . . . . 39

2.1 .2 BICM Components . . . . . . . . . . . . . . . . . 41

2.2 BICM Capacity Limits . . . . . . . . . . . . . . . . . . . 43

2.2.1 The Unconstrained Shannon Limit . . . . . . . . . . . . 43

2.2.2 Capacity Calculation for BICM . . . . . . . . . . . 44

2.2.3 BICM Limits for Uniform QAM Constellations . . . . . 45

2.2.4 Extension to MIMO-BICM Systems . . . . . . . . . 48

2.3 Single-Antenna Receivers . . . . . . . . . . . . . . . . . . 49

2.3.1 Demapping Algorithms . . . . . . . . . . . . . 50

2.3 .2 Signal Quantization . . . . . . . . . . . . . . 53

2.4 Multi-Antenna Receivers . . . . . . . . . . . . . . . . 55

2.4.1 ML and Max-Log Demappers . . . . . . . . . . 56

2.4.2 ZF and MMSE Detectors . . . . . . . . . . . . . 57

2.4.3 Sphere Decoding Techniques . . . . . . . . . 58 
3 Optimization and Performance Evaluation of Non-Uniform Cons$\begin{array}{ll}\text { tellations } & \mathbf{6 1}\end{array}$

3.1 Non-Uniform Constellations Optimization . . . . . . . . . . . 61

3.1.1 One-Dimensional Non-Uniform Constellations . . . . . . 62

3.1.2 Two-Dimensional Non-Uniform Constellations . . . . . 70

3.1.3 BICM Capacity Improvements . . . . . . . . . . . 75

3.2 Non-Uniform Rotated Constellations . . . . . . . . . . . . . . 77

3.2.1 Optimization Before Rotation . . . . . . . . . . 78

3.2.2 Optimization with Additional Rotation . . . . . . . . 80

3.3 Application of NURC to Multi-RF Techniques . . . . . . . . . 82

3.4 Performance Analysis Based on Physical Layer Simulations . . 84

3.4.1 Non-Uniform Constellations Gain . . . . . . . . . . . . . 85

3.4.2 Rotation Gain . . . . . . . . . . . . . . . 87

3.4.3 Non-Uniform Rotated Constellations with Multiple RF Channels ................ 87

3.5 Conclusion ....................... . . 94

4 Low-Complexity Demapping and Quantization Algorithms $\quad 97$

4.1 Demapping Complexity at the Receiver . . . . . . . . . . . . . 98

4.2 Low-Complexity Demapping Algorithm . . . . . . . . . . . . . 100

4.2.1 Quadrant Search Reduction (QSR) . . . . . . . . 100

4.2.2 Condensed Symbols Reduction (CSR) . . . . . . . . . 102

4.2.3 Quadrant Condensed Search Reduction (QCSR) . . . . 104

4.3 Performance Evaluation: Minimum Number of Distances . . . . 105

4.3.1 Calculation of the Minimum Number of Distances . . . 105

4.3.2 Performance Loss with Alternative Channel Models . . 108

4.3.3 QCSR with Non-Uniform Rotated Constellations . . . . 110

4.4 Digital Quantization of LLR and I/Q Components . . . . . . . 111

4.4.1 System Model and Considered Scenario . . . . . . . . . 112

4.4.2 Quantization of Log-Likelihood Ratios . . . . . . . . . . 115

4.4.3 Quantization of I/Q Components . . . . . . . . . . . 118

4.5 Quantization Loss vs. Time De-Interleaving Memory Trade-Off 121

4.5.1 Performance Loss of LLR Quantization . . . . . . . . . 121

4.5.2 Performance Loss of I/Q Components Quantization . . 124

4.5.3 Time De-Interleaving Memory Requirements . . . . . . 125

4.6 Conclusion .................... 127

5 Non-Uniform Constellations for MIMO Communications 129

5.1 Multi-Antenna Non-Uniform Constellations . . . . . . . . . . . 130

5.1.1 Preliminary Design . . . . . . . . . . . . . . 131

5.1.2 Re-optimization without Power Imbalance . . . . . . . . 138 
5.1.3 Re-optimization with Power Imbalance . . . . . . . . . . 140

5.2 Performance Evaluation of Multi-Antenna Non-Uniform Constellations . . . . . . . . . . . . . . . . 142

5.2.1 Transmission without Power Imbalance . . . . . . . . . 142

5.2.2 Transmission with Power Imbalance of $6 \mathrm{~dB}$. . . . . . . 143

5.3 Demapping Complexity Analysis . . . . . . . . . . . . . . 145

5.4 Fixed Sphere Decoder for Two-Dimensional NUCs in MIMO . 146

5.4.1 Successive Interference Cancellation . . . . . . . . . . . 147

5.4.2 Voronoi Regions Selection Algorithm . . . . . . . . . . . 147

5.4.3 Resolution vs. Performance Trade-Off . . . . . . . . . . 151

5.5 Conclusion . . . . . . . . . . . . . . . . . . . 152

6 Conclusions and Future Work 153

6.1 Concluding Remarks . . . . . . . . . . . . . . . . . . . . 154

6.1.1 Non-Uniform Constellations Optimization . . . . . . . . 154

6.1.2 Complexity Implications at the Receiver . . . . . . . . . 155

6.1.3 Multi-Antenna Optimization and Complexity Reduction 157

6.2 Constellation and CR Recommendation . . . . . . . . . . . . 158

6.3 Future work . . . . . . . . . . . . . . . . . . 161

6.3.1 High-Order Two-Dimensional Non-Uniform Constellations 161

6.3.2 Further Optimization for MIMO Systems . . . . . . . . 161

6.3.3 The Future of Broadcasting: Constellations for 5G Communications ................ 161

A Physical Layer Simulator $\quad \mathbf{1 6 3}$

A.1 Transmitter Block Diagram . . . . . . . . . . . . . . . 163

A.2 Receiver Block Diagram . . . . . . . . . . . . . . . . 168

A.3 Channel Models . . . . . . . . . . . . . . . . . . . . . 170

B Optimization Algorithm $\quad \mathbf{1 7 5}$

B.1 Nelder-Mead Simplex Method . . . . . . . . . . . . . . . . 175

B.2 Application to Use Cases Considered . . . . . . . . . . . . . . . 179

$\begin{array}{lr}\text { Acronyms } & 183\end{array}$

$\begin{array}{lr}\text { References } & 187\end{array}$ 


\section{Chapter 1}

\section{Introduction}

\subsection{Evolution and New Challenges of Digital Terrestrial Broadcasting}

Television (TV) is one of the most popular and extended telecommunication systems in the world. Commercial TV as it is known today began in the late 1940s. Its implementation introduced dramatic social changes and facilitated the appearance of new business models. TV has coexisted with society during more than 70 years, experiencing big transformations such as the transition from black and white to color, or from analog to digital. With the arrival of flat-screen displays, Digital Terrestrial Television (DTT) communications and video compression systems, TV has experienced a high-speed and large evolution in the 21st century.

The switch from analog to digital entailed several advantages such as the transmission of noise-free high-quality video and audio, a larger exploitation of the Radio Frequency (RF) spectrum, the delivery of multilingual audio tracks, subtitles and interactivity, or the use of a flexible network with configurable parameters such as transmission power, capacity or quality of service. Currently, DTT is the main TV system adopted in many European countries including the United Kingdom, France, Spain, Portugal and Italy, being ahead other services such as cable or satellite TV. DTT systems are capable of providing a specific set of services without any restriction in the number of users [1]. DTT allows for an efficient delivery of free-to-air content to large audiences with a guaranteed quality of service, and provides a near universal coverage of over $98 \%$ population [2]. With DTT, the Ultra-High Frequency (UHF) spectrum needed to transmit a single analog channel is used to carry several multiplexed digital 
services. In other words, the same set of services can be transmitted using just a reduced part of the spectrum available. As a consequence, the spectral efficiency increase offered by DTT systems attracted emerging technologies such as Long Term Evolution (LTE) to request part of the UHF spectrum.

\section{First and Second Digital Dividends}

In the World Radiocommunication Conference (WRC)-07, the International Telecommunications Union (ITU) decided to allocate the upper part of the TV broadcasting band to International Mobile Telecommunications (IMT) technologies, giving room to which is known as Digital Dividend (DD) [3]. Regions 1 (Europe and Africa) and 3 (Asia) allocated the $800 \mathrm{MHz}$ band (790-862 $\mathrm{MHz}$, channels 61-69) for fourth generation (4G) LTE services, and Region 2 (America) allocated the $700 \mathrm{MHz}$ band (698-806 MHz, channels 52-69). In the WRC-12, the ITU concluded with a decision to allocate additional UHF spectrum to mobile services. This situation will remain for more than 10 years, since in the WRC-15 it was decided that there will not be any change to the allocation in the 470-694 MHz band for the time being.

The new mobile allocation, also known as Second Digital Dividend (DD2), is to be made in Region 1 in the $700 \mathrm{MHz}$ band. The main difference compared to the $800 \mathrm{MHz}$ band lies in the fact that the Uplink (UL) is located in the lower part, instead of the Downlink (DL). For most countries, releasing the 700 $\mathrm{MHz}$ band will require a new re-tune of existing DTT networks. Implementing the DD2 within ITU Region 1 affects up to eleven more DTT channels (49-60), creating a number of challenges. Since cellular terminals are closer to the DTT receivers than base stations, interference issues may be relevant in the $700 \mathrm{MHz}$ band [4]. The DD2 is particularly problematic in countries where terrestrial $\mathrm{TV}$ is the main distribution platform.

The DD2 arises as a turning point for introducing new DTT systems and video compression standards, in order to increase the network spectral efficiency and provide new services such as Ultra High-Definition TV (UHDTV). In fact, reference [5] presents an overview of the upcoming television broadcast spectrum incentive auction in the United States, reviews the potential plans for the $600 \mathrm{MHz}$ band, and discusses the opportunities that could bring the use of new digital terrestrial television specifications.

\section{Initial DTT Technologies}

Nowadays, several first generation DTT technologies are in place over the world, such as Advanced Television Systems Committee (ATSC) in North America and South Korea [6], Integrated Services Digital Broadcasting - Terrestrial (ISDB-T) in Japan and South America [7], or Digital Terrestrial Multime- 
dia Broadcast (DTMB) in China [8]. Although these technologies are utilized in many countries, Digital Video Broadcasting - Terrestrial (DVB-T) is the most widely implemented DTT standard in the world. DVB-T permits to configure a number of parameters in order to adapt the system to a particular network and transmission requirements. The DVB-T specification provides bit rates ranging from 4 to 30 Mbps [9]. DVB-T, together with ISDB-T and DTMB specifications, is based on the multi-carrier Orthogonal Frequency-Division Multiplexing (OFDM) modulation [10]. All data carriers are modulated using different uniform Quadrature Amplitude Modulation (QAM) constellations, that is, QPSK, 16QAM or 64QAM. DVB-T permits to use several Coding Rates (CR), Guard Intervals (GI) or Fast Fourier Transform (FFT) sizes to adapt the signal. However, first generation standards are still far from the theoretical Shannon capacity limit [11]. Motivated by technological progress and new advanced techniques, different standardization forums decided to develop next-generation DTT specifications.

\section{Next-Generation Digital Terrestrial Broadcasting}

The DVB forum developed a second generation standard, known as DVB Terrestrial Second Generation (DVB-T2) [12], which provides a 50\% increase of spectral efficiency compared to DVB-T. It permits to use a more advanced configuration of parameters to transmit, including a wider set of coding rates. DVB-T2 employs a serial concatenation of inner Low Density Parity Check (LDPC) codes and outer Bose Chadhuri Hocquenghem (BCH) codes. It is also based on the multi-carrier OFDM modulation, and permits the use of a single or multiple Physical Layer Pipes (PLP) that allow to transmit different services with specific capacity and robustness. The DVB-T2 specification provides an extended interleaving that increases robustness in both time and frequency domains. It also supports the concept of Rotated Constellations (RC) and includes an additional 256QAM constellation.

Standardization activities were also addressed on the development of mobile broadcasting systems, despite the lack of market and financing needed [13]. The handheld evolution of DVB-T2, Digital Video Broadcasting - Next Generation Handheld (DVB-NGH), is the state-of-the-art standard for DTT mobile communications, and includes some of the most advanced transmission techniques to cope with adversities and characteristics of mobile channels [14]. It was the first broadcasting system including the concept of onedimensional Non-Uniform Constellation (NUC), for 64 and 256 orders. Another relevant technique included in DVB-NGH was the use of Multiple-Input Multiple-Output (MIMO). The concept of MIMO is based on the use of several transmit and receive antennas to transmit different signals at the same 


\section{CHAPTER 1. INTRODUCTION}

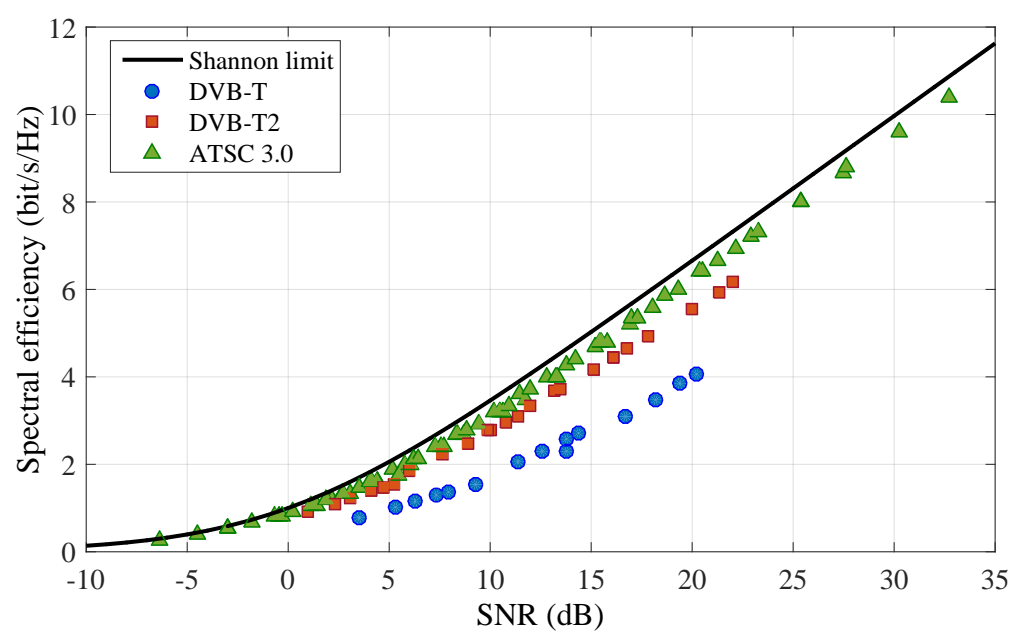

Figure 1.1: Spectral efficiency of DVB-T, DVB-T2 and ATSC 3.0 specifications compared to the Shannon capacity limit, for AWGN channel.

time. The transmission of two or more streams in parallel permits to increase transmission capacity, but also robustness.

The use of new digital standards along with more efficient video coding arises as an opportunity to introduce two new features simultaneously, guaranteeing an efficient use of the remaining spectrum. The state-of-the-art terrestrial broadcasting standard, ATSC - Third Generation (ATSC 3.0), tries to solve this problem. It focuses on shortening the gap to Shannon limit through more efficient constellations and very-low coding rates, the aggregation of multiple RF channels in which is known as Channel Bonding (CB), or the combined provision of fixed and mobile services, among others [15]. Fig. 1.1 shows the performance achieved with ATSC 3.0 in terms of spectral efficiency (bit $/ \mathrm{s} / \mathrm{Hz}$ ) vs. SNR (dB), for AWGN channel. ATSC 3.0 is also compared with some of its antecessors, i.e. DVB-T and DVB-T2. ATSC 3.0 includes some of the newest techniques developed in broadcasting such as MIMO or Layered Division Multiplexing (LDM) [16]. LDM enables the efficient provision of mobile and fixed services by superposing two independent signals with different power levels in a single RF channel. With ATSC 3.0, it is also possible to split service data across two RF channels, so that peak data rate can be doubled. ATSC 3.0 also includes two-dimensional (2D) NUCs from 16 to 256 constellation symbols, and 1D-NUC for new high-orders such as 1024NUC (or 1kNUC) and 4096NUC (or $4 \mathrm{kNUC)}$ [17]. 


\subsection{Preliminaries}

The problem of designing a system that operates close to the unconstrained Shannon theoretical limit has been one of the most important and challenging problems in information/communication theory [11]. As reference [18] states, one straightforward answer to the question of how to efficiently transmit more than one bit per symbol is a Coded Modulation (CM) scheme, where the channel encoder is combined with a modulator and several bits are mapped into a symbol. What is not straightforward is how to configure a system that operates close to the Shannon capacity limit, but with low complexity. In [19], the idea of jointly design the channel encoder and modulator was firstly proposed, which inspired several CM schemes such as trellis-coded modulations [20] or multilevel codes [21]. Since both schemes aimed to maximize the Euclidean distances to symbols, they performed considerably well for the AWGN channel. However, their performance in fading channels is insufficient.

Bit-Interleaved Coded Modulation (BICM) is the pragmatic approach for combining channel coding and digital modulations in fading transmission channels. The core of the BICM encoder consists of the serial concatenation of a Forward Error Correction (FEC) code, a Bit Interleaver (BIL), and a memoryless mapper that assigns blocks of bits to constellation symbols. BICM aims to increase the coding diversity (the key performance measure in fading channels) and therefore, outperforms the CM schemes mentioned above, for this scenario [22], [23]. BICM is very robust to variations of the channel characteristics, and very attractive from an implementation point view due to its low complexity and flexibility, since the channel encoder and the modulator can be selected independently. This dissertation focuses on modulation, one of the most important parts to consider when designing BICM systems.

With uniform QAM constellations, the symbols are regularly spaced in the constellation diagram, i.e. with the same distance between symbols in both in-phase (I) and quadrature-phase (Q) components. This uniform distance allows for an easy design of the modulation module that also needs a very simple demapping process. As a main drawback, it entails a performance loss due to the two constraints imposed by assuming a rectangular shape and equally spaced levels [17]. This difference to the capacity limit also increases with the modulation order. For that reason, NUCs can be used to reduce the gap between the BICM capacity of uniform Gray-labelled constellations and the unconstrained Shannon limit. With NUCs, the constellation symbols are optimized by means of signal shaping techniques to provide an improved performance compared to uniform QAM constellations. These constellations are designed for a particular SNR and channel model. As stated before, NUCs are categorized into two different groups: 1D-NUCs and 2D-NUCs. 1D-NUCs 

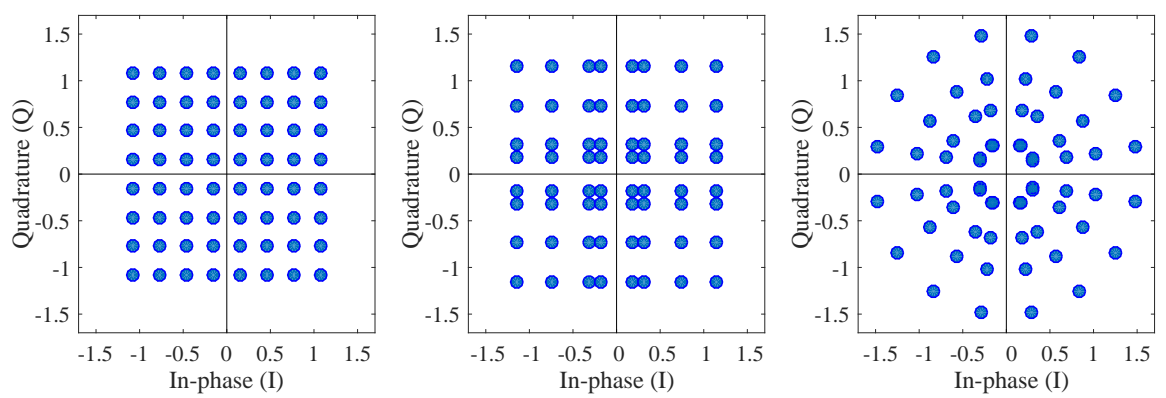

Figure 1.2: Different constellations considered. Uniform 64QAM (left), 1D-64NUC (center) and 2D-64NUC (right), optimized for a SNR of $10 \mathrm{~dB}$ and AWGN channel.

have a squared shape with non-uniform distance between constellation symbols, while 2D-NUCs are designed by relaxing the square shape constraint, with a better SNR performance than 1D-NUCs but with higher demapping complexity. Fig. 1.2 depicts two examples of 1D- and 2D-64NUCs optimized for a SNR of $10 \mathrm{~dB}$ in a Additive White Gaussian Noise (AWGN) channel. QAM is also shown for comparison.

At low SNRs, NUCs collapse into lower orders of constellation, where almost identical symbols are grouped in clusters. These constellations are known as condensed NUCs. With the condensation, the Most Significant Bits (MSB) provide similar robustness as the positions of low-order constellations. On the other hand, the Least Significant Bits (LSB) cannot be resolved from the overlapping points, since they offer a very weak information, close to zero [17].

Note that the gain provided by NUCs becomes almost non-existent at high SNRs, especially when optimizing for fading channels [24]. In this particular case, Signal Space Diversity (SSD) techniques can be used to improve the overall system performance. The most widespread SSD technique is the use of RC. With RC, a certain rotation angle is applied to the constellation, so that the binary information is transmitted simultaneously in different I and Q components. In order to ensure that each component undergoes independent fading, a component interleaver is needed after the rotation [25]. Moreover, RCs become especially effective when using multi-RF techniques such as CB or Time-Frequency Slicing (TFS), providing very high performance gains. TFS was proposed in DVB-T2 and adopted in DVB-NGH. With TFS the data is transmitted in a slot-by-slot manner by frequency hopping across an RF-Mux of two or more RF channels (in practice, up to 6). On the other hand, CB consists of splitting service data across two RF channels, so that peak data rate can be 
doubled. In such case, it is desirable that each component is transmitted in a different RF channel.

One of the main drawbacks when using 2D-NUCs is the demapping complexity. The complexity with QAM constellations and 1D-NUCs can be drastically reduced by using a one-dimensional demapper [26], thanks to the squared shape of these constellations. This demapper, nevertheless, cannot be used with 2D-NUCs. In this case, demappers need to evaluate the distances in both dimensions (real and imaginary parts) for all possible symbols. The same occurs with RCs, where the binary information is transmitted simultaneously in different I and Q components, and the demapper has to consider all symbols in two dimensions, regardless of the constellations shape.

Another important bottleneck in receivers is the quantization of digital signals. As reference [27] mentions, quantization is a basic operation that is applied in digital receivers, and permits transformation of continuous to discrete digital signals to be processed, transmitted and stored in conventional digital processors. In DTT receivers, memory requirements at the Time DeInterleaver (TDIL) are much higher than mobile communications [28]. The TDIL interlaces multiple constellation symbols to increase the signal time diversity, and hence to increase the protection against time fading. The memory required to store all the received symbols of the block depends on the number of quantization levels and their distribution, and consequently on the use of these new constellations.

NUCs can be also efficiently used for multi-antenna configurations. By using more than one single antenna in both the transmitter and the receiver, MIMO improves the transmission robustness via additional spatial diversity, or increases capacity by sending multiple data streams in the same bandwidth via spatial multiplexing [29]. Fig. 1.3 shows a generic block diagram of a MIMO system with $N_{T}$ transmit and $N_{R}$ receive antennas. While Single-Input Multiple-Output (SIMO) exploits diversity and array gains, and Multiple-Input Single-Output (MISO) only retains the spatial diversity gain, the Spatial Multiplexing (SM) gain is achieved only when using two or more transmit and receive antennas. The SM allows MIMO to overcome the capacity limits of SISO communications without any increase in the total transmission power or channel bandwidth [30]. An impending problem of MIMO is the extremely high demapping complexity when using optimum Maximum Likelihood (ML) or max-log demappers, since it grows exponentially with the number of antennas and the constellation order. To reduce this complexity, different suboptimum demappers have been proposed in the current literature. With the use of suboptimum demappers, the complexity can be drastically reduced, while maintaining a good performance, compared to the optimum ML demapper. 


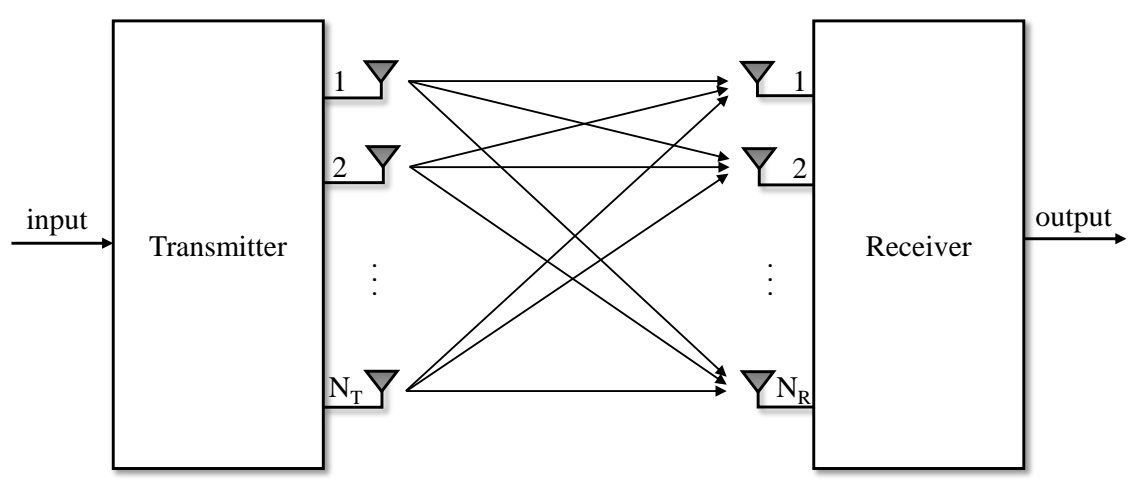

Figure 1.3: Block diagram of a generic MIMO system, with $N_{T}$ transmit and $N_{R}$ receive antennas.

\subsection{Research Challenges on Non-Uniform Cons- tellations}

This thesis focuses on Non-Uniform Constellations (NUC), one of the most innovative techniques included in last broadcasting specifications. Two main key performance indicators define these constellations, depending on the number of dimensions in which they are optimized:

- Improved SNR performance.

- Demapping complexity.

In this dissertation, the implementation aspects of these constellations are investigated from both transmitter and receiver point of views. The challenge is to design constellations that increase the system performance, and to use simplified sub-optimum demappers that reduce the demapping complexity at the receiver without any significant loss. Different orders of 1D- and 2D-NUCs are designed. The optimization process is performed so the BICM capacity is maximized for each considered configuration. This is equivalent to an optimization process in which SNR values are reduced, but with reduced computation complexity. The use of rotated NUCs combined with multi-RF techniques is another aspect to take into account. At the receiver, optimum demappers calculate the distances from the received signal to all constellation symbols. In that sense, a suboptimum algorithm with very low complexity is proposed to reduce the number of distances to compute, while keeping a good performance. Another problem when using NUCs is signal quantization. Quantization has 
been always related in the literature with traditional communications systems, and therefore, uniform QAM constellations. Hence, it is necessary to explore how quantization values are modified when using NUCs, and to provide reduced algorithms that simplify the number of operations required.

As a matter of fact, the use of NUCs opens a wide range of possibilities to explore. In this thesis, NUCs are also effectively combined with advanced signal processing techniques such as RC or multi-RF averaging. Rotated NUCs may become especially effective when using techniques that combine the information transmitted over several RF channels. NUCs can be also effectively combined with MIMO. The combination of NUCs and MIMO is a relatively new research topic in the literature, and further investigation is needed, both at the transmitter and receiver sides.

\subsection{Objectives and Scope}

The main objective of this dissertation is to investigate and assess the performance of the combination of non-uniform constellations with advanced signal processing techniques, with the goal of maximizing the spectral efficiency and signal robustness, while reducing the overall demapping complexity in next-generation DTT systems. In particular, the following are the partial objectives of the dissertation:

\section{Single-Antenna Transmission}

- To design and optimize NUCs, assessing their performance gain compared to uniform QAM constellations. The aim is to obtain different constellations that provide additional shaping gain, which allows reception at lower SNRs. This study also aims to identify the capacity and performance gains over QAM, using ideal receivers. NUCs are designed for different constellation orders and SNR values, directly related to specific coding rates. Different channel models, types of reception, and the use of an optimization algorithm that better approaches the final solution are also investigated.

- To analyze the use of rotated 2D-NUCs combined with multiRF technologies. Rotation gains for a single RF transmission (default mode in broadcasting) are first investigated. Afterwards, rotated NUCs are analyzed in connection with multi-RF techniques, where they may become a good solution to increase robustness. This dissertation provides an optimization method, in which the rotation angle is considered as 
an additional variable. The potential NUC rotation gains in multi-RF transmissions are also investigated.

\section{Single-Antenna Reception}

- To provide a generic low-complexity demapping algorithm for 2D-NUCs. One of the bottlenecks in real receiver implementations is the use of demappers that calculate the distances to all constellation symbols. It is necessary to reduce the demapping complexity in order to use highorders of constellation, while obtaining a reduced performance loss. Two different strategies can be considered. The first one takes advantage of the symmetry that constellations provide, and the second exploits the condensation of some constellations, whose constellation symbols almost repeat the same position.

- To evaluate the influence of NUCs on soft-quantized LLR and I/Q components. The use of NUCs instead of QAM implies the use of non-uniform complex values in the I/Q space, and therefore modified output LLR values. The aim is to compute memory savings of this new type of constellation, considering both uniform and non-uniform quantization. The objective is to reduce memory while keeping a good performance. An additional objective is to study the performance and in-chip memory trade-off of soft-quantized receivers designed.

\section{Multi-Antenna Communications}

- To assess the use of multi-antenna MIMO systems for NUC optimization. In the ATSC 3.0 standardization, constellation designed for SISO were also adopted for MIMO. However, since constellations are optimized for a particular SNR and channel model, a new re-optimization process may be needed. Moreover, NUCs can be optimized for MIMO also taking into account the new parameters introduced by these systems, such as the power imbalance or the cross-polar discrimination between antennas.

- To propose a new suboptimum algorithm for an efficient demapping of 2D-NUCs in MIMO. At the receiver, the number of possible received symbols grows exponentially with the number of antennas and the constellation order. The objective is to propose an efficient approach that reduces the demapping complexity at the receiver. The proposed demapping technique needs also to be compared with optimum demappers, in terms of distances to be computed and SNR performance. 


\subsection{State-of-the-Art}

\section{Bit-Interleaved Coded Modulation}

BICM was first introduced by reference [31] in 1992, where it was shown that the performance of coded modulation over fading channels is improved with a $\log (M)$ bit interleaver at the encoder output, and by using soft-decision metrics as inputs to the decoder. However, the discussions provided in it were restricted to a rate $2 / 3$ coded system with a 8 -PSK modulation. In [22], the informationtheoretical view of BICM and the necessary tools for evaluating its performance were provided. More recent references [18] and [32] paid special attention to different bit-to-symbol labeling strategies. Both references analyzed the BICM capacity vs. Signal-to-Noise Ratio (SNR), showing that for a specific labeling this relation is not a function, where the same SNR maps to more than one capacity value.

Nowadays, state-of-the-art BICM broadcasting systems are currently very close to the Shannon limit. In [33], the structure of the BICM module in DVBT2 was widely described, which is formed by the serial concatenation of a FEC code, a bit interleaver, and a QAM mapper. It also presented the modifications and the new elements that were introduced in DVB-NGH. A more recent study [34] summarizes and expounds the choices made for the BICM part of the ATSC 3.0 standard. The result is a BICM chain that provides the largest operating range (more than $30 \mathrm{~dB}$, with the most robust mode operating below $-5 \mathrm{~dB}$ of SNR), and the highest spectral efficiency compared to any digital terrestrial system so far. FEC coding techniques employed in this standard are further explained in [35]. This reference presents two different structures of LDPC codes: irregular repeat accumulate structure and multi-edge type structure, which are used for a wide range of coding rates.

\section{Non-Uniform Constellations}

Previous works in the literature outline the design of NUCs and their potential gains. In 1974, reference [36] noted the capacity shortfall for uniform QAM constellations, and introduced the non-uniform concept, obtaining several constellations which offer a capacity improvement. It was the first approach to the constellations nowadays known as NUCs, and constituted the basis for all the studies performed up to now. More recent studies [37] and [38] tackled the optimization of one-dimensional NUCs with 16 to 1024 symbols, in an AWGN channel. Here, the concept of a condensed constellation is presented for the first time. In [24], high-order 1D- and 2D-NUCs are optimized up to 1024 symbols as well, also for AWGN channel. The presented constellations approach the Shannon limit up to $0.036 \mathrm{bit} / \mathrm{s} / \mathrm{Hz}$ at $29 \mathrm{~dB}$ of SNR, corresponding to a short- 
coming of approximately $0.1 \mathrm{~dB}$. In [39], high-order NUCs with constellation sizes of up to 4096 symbols are also investigated.

Different NUC optimizations and analysis were also made for specific systems and techniques. Reference [40] presented a complete analysis of the DVBT2 system performance when using the proposed 2D-NUCs, and compared with QAM constellations. Reference [17] studies the use of NUCs within ATSC 3.0, which is the first major broadcasting standard that completely uses this type of constellations. Both 1D- and 2D-NUCs are considered in this work. Results in this reference show that shaping gains of more than $1.5 \mathrm{~dB}$ are possible, which can be seen as a major step towards the ultimate limits of broadcast communications. In [41], the authors investigate the effect of NUCs on the performance of Iteratively Decoded Bit-Interleaved Coded Modulation (BICM-ID) systems with different FEC coding schemes, in which NUCs also provide additional gains up to $0.2 \mathrm{~dB}$. In [42], constellations are designed for the DVB-T2 L1Post signal, providing a potential improvement of $0.55 \mathrm{~dB}$ for the overall signaling performance using $64 \mathrm{NUCs}$, for AWGN channel. Slightly lower gains are also obtained for Rayleigh channel.

Different demappers can be also found in the current literature to reduce complexity of 2D-NUCs. A first approach was provided in [43], which analyzes the quadrant symmetry of the constellations, in order to pre-compute the LLR values in a Look-Up Table (LUT) and completely avoid the demapping process. A demapper exploiting the condensation of NUCs was first proposed in [37]. It works especially well at low SNRs, where some of the constellation symbols almost repeat the same position in the I/Q plane. Such condensed constellations lead to reduce the number of effective bits. Since Log-Likelihood Ratios (LLR) of the least significant bits are negligible, these non-effective bits are not necessary to be decoded. This demapper was further analyzed in [44]. Following the same strategy, authors in [45] propose high order low-complexity 2D-NUCs with cardinality points 1024 and 4096. Low complexity is achieved via condensation of the closest symbols, and the improvement in the performance compared to 1D comes from the two-dimensional optimization.

\section{Combination with Advanced Signal Processing Techniques}

NUC optimization can be particularized for different techniques, as long as the SNR or channel conditions under study are accordingly modified. For instance, authors in [46] propose a new strategy to design NUCs for future television broadcast systems supporting LDM. The proposed method optimizes the BICM capacity simultaneously for two configurations performing at different SNR values [47]. LDM has been adopted for the first time in ATSC 3.0, and defined in [16]. In [48], authors illustrate a performance comparison between 
LDM and Frequency Division Multiplexing (FDM)/Time Division Multiplexing (TDM), in terms of capacity-coverage tradeoff of the mobile service. For a given reduction of fixed service capacity, authors keep the coverage constant.

Regarding constellation rotation, it was first presented in [49], and further studied in [50]. In both studies, very high diversity orders were achieved, obtaining an almost Gaussian performance over the fading channel under study. It was shown that the average mutual information between the signal after rotation and the signal before or after the demapper varies with the rotation angle. Reference [51] provided substantial gains with respect to classical BICM broadcasting systems, under several channel conditions. The resulting improvement in performance varies from $0.2 \mathrm{~dB}$ to several $\mathrm{dBs}$, depending on the order of the constellation, the $\mathrm{CR}$ and the channel model. Thanks to the advantages studied, this technique was adopted in the DVB-T2 standard. In [25] and [52], authors also tackled the use of RCs in broadcasting systems. In particular, the manuscript investigates the potential gains that can be obtained with rotated constellations in DVB-NGH.

Several low-complexity demappers have been proposed for the use of RCs as well. The demapper proposed in [53] and [54] takes advantage of the symmetry that constellations provide, selecting a reduced cluster of symbols to compute the LLRs. It reduces up to $78 \%$ the number of required operations with almost no performance degradation compared to optimum demappers. Another technique is also provided in [55]. In the proposed approach, only a subset of the constellation symbols close to the received samples is considered for demapping. The concept is similar to a sphere demapper [56], which also selects a cluster of symbols to compute the LLRs. An additional complexity-reduced max-log demapper for RCs was proposed in [57]. The proposed demapper allows to find the ML symbols with a search spanning, calculating only $\sqrt{M}$ signal constellation symbols and guaranteeing the same LLR metrics as the original max-log demapper.

Constellation rotation can be efficiently combined with multi-RF techniques such as TFS or CB [58]. Reference [59] provides a brief introduction to TFS and its realization within the standard DVB-T2. Simulation results and theoretical analysis also show the potential gains and limits of TFS. The use of this multi-RF technique is also studied in [60]. The papers investigate the potential advantages of TFS by means of field measurements as well as simulations and discusses practical implementation aspects and requirements regarding transmission and reception. A complete study on this field can be also found in [61], which shows that, in addition to a potential capacity gain, TFS can provide very important coverage gains of around $4.5 \mathrm{~dB}$ for a $4 \mathrm{RF}$ channels multiplex, according to field measurements. On the other hand, the use of $\mathrm{CB}$ in ATSC 3.0 is explained in [62]. In [63], CB is combined with LDM. A more 


\section{CHAPTER 1. INTRODUCTION}

recent approach for multi-RF combining has been proposed in [64], where all frequencies within the UHF band are used on all transmitter sites. This approach allows for a dramatic reduction in fundamental power/cost and about $37-60 \%$ capacity increase. Note that the use of rotated NUCs has not yet been studied in connection with multi-RF techniques, where they may become a good solution to increase robustness. In this dissertation, rotated 2D-NUCs are designed using a new method, in which the rotation angle is considered as an additional variable. The potential gains obtained, both in single-RF and multi-RF transmissions, are also provided.

\section{Signal Quantization}

Quantization has been widely studied in the literature. In [65], the authors discuss the problem of the minimization of the distortion of a signal by a quantizer when the number of output levels of the quantizer is fixed. Equations are derived for the parameters of a quantizer with minimum distortion, and an algorithm is developed to simplify their numerical solution. Optimization under the restriction that both input and output levels are equally spaced is also treated. In [66], and in a more extended work [67], the whole concept of quantization is explained, surveying the fundamentals of the theory and many of the popular techniques for quantization. Reference [68] considers mutual-information-optimal quantization of LLRs. An efficient algorithm, reminiscent of the famous Lloyd-Max algorithm, is presented for the design of LLR quantizers based either on the unconditional LLR distribution or on LLR samples, which can be used to design LLR quantizers during data transmission. In [69], authors reduce the memory size with a negligible increase in computational complexity by quantizing LLRs with bit-specific quantizers and compressing the quantized output. Numerical results show that the proposed solution enables a memory saving up to $30 \%$. Reference [27] presents two different quantization methods for multi-antenna broadcasting receivers, using QAM constellations. In this reference, the influence of quantization on LLR values and I/Q symbols is evaluated. The numerical evaluations show that non-uniform quantizers adapted to the signal statistics provide significant improvements in terms of system performance or alternatively in-chip memory savings.

\section{MIMO Signal Processing}

As reference [70] states, the use of MIMO for terrestrial broadcasting in the UHF band requires co-located antennas with cross-polar polarization (horizontal and vertical) in order to maintain the SM capability in Line-of-Sight (LoS) condition. The biggest difference when comparing MIMO broadcasting and 
unicast transmissions, is that no Channel State Information (CSI) is shared between transmitter and receiver. This condition logically reduces the potential gain. Nevertheless, even under this condition the MIMO broadcasting gain is still very large, especially for high SNRs [29]. An efficient option to improve the SNR in this case is the use of a channel precoder as presented in [71]. This precoder is optimized according to broadcast channel statistics by linearly combining the data streams across the transmit antennas. The proposed precoder can provide significant capacity improvements for users with a strong LoS component and correlated MIMO paths, and is potentially transparent to consumer receivers. Another precoder was proposed in [72]. It is designed using amplitude and phase parameters, and is suitable for practical environments. The proposed scheme is especially beneficial in MIMO channels with random phase correlation. Different field tests have been also performed in [73] to demonstrate the use of MIMO for DTT. These $2 \times 2$ MIMO field tests involved terrestrial $8 \mathrm{~K}$ transmissions over a single RF channel. The SNR degradation compared to laboratory measurements was under $3 \mathrm{~dB}$ even in Non-Line-of-Sight (NLoS) conditions. Reference [74] provides a performance comparison of soft-output and hard-output demodulators in the context of non-iterative MIMO systems. Results obtained give new insights for a MIMO-BICM system design, including antenna configuration, constellation symbols, and demodulation for a specific CR.

MIMO has been adopted in new generation DTT specifications such as DVB-NGH and ATSC 3.0, as an optional technology. The DVB-NGH technical specification [14] was the first terrestrial broadcasting standard supporting MIMO [75]. In DVB-NGH, field measurements were also used to develop representative channel models for mobile and fixed receptions, which can be found defined in [27]. These channel models are used in this dissertation, and are detailed in Appendix A. MIMO may become a key technology for ATSC 3.0, since the DVB-T2 standard [12] does not include this feature. Reference [76] provides an overview of the optional MIMO antenna system adopted in ATSC 3.0, intended for $2 \times 2$ cross-polarized MIMO. This means that at least two antennas with horizontal and vertical polarization are present at both transmitter and receiver sides. The MIMO scheme adopted in ATSC 3.0 re-uses the SISO antenna baseline constellations, and hence it introduces the use of MIMO with NUCs. In [77], simulations demonstrated that NUCs are advantageous for MIMO, compared with conventional uniform QAM constellations. NUCs were implemented on both MIMO-OFDM modulator and demodulator prototypes, and investigated under laboratory measurements and field testing. Results showed that high-order NUCs improved the required SNR by $1 \mathrm{~dB}$, compared to QAM. However, the use of this constellations is not optimum for MIMO, since they were optimized for SISO, with a different SNR and chan- 


\section{CHAPTER 1. INTRODUCTION}

nel model. This dissertation provides additional constellations optimized for MIMO, and compares their performance with original constellations optimized for SISO.

As main disadvantage, the use of MIMO drastically impacts the demodulation at the receiver, being necessary the use of suboptimum demappers to reduce complexity. In [78], the presented demapper applies a simplification when calculating the Euclidean distances needed to compute the LLRs. This step avoids almost all multiplications and presents a performance loss under $0.1 \mathrm{~dB}$. Reference [79] presents the Decision Threshold (DT) algorithm, which only requires a single Euclidean distance in the I/Q components to calculate each LLR, regardless of the number of constellation symbols. The computational complexity is drastically decreased with DT, with a maximum SNR degradation of approximately $0.4 \mathrm{~dB}$. Results show that the LLR approximation is applicable to transmission systems with high-orders of 1D-NUC. In [80], additional commonly used solution techniques are reviewed, and their computational complexity is discussed. Among heuristic algorithms, the authors focus on cancelling techniques, and their fast implementations based on linear estimation theory. Some examples are Zero Forcing (ZF), nulling and cancelling, or nulling and cancelling with optimal ordering.

Another possibility to reduce complexity is the use of an Sphere Decoder (SD). The original idea behind SD is to reach the optimum demapping solution with lower complexity than the exhaustive search. This is done by looking for the optimum solution just within a subset of the total possible symbols. As explained in [81], this subset is a hyper-sphere centered at the received signal vector with a certain radius. All SD insights are detailed graphically and mathematically in [82]. SD involves a high number of variations. For instance, Soft Fixed-Complexity Sphere Decoders (SFSD) represent a good approach, since they achieve a sub-optimum max-log performance, while keeping a very reduced fixed complexity that is reasonably close to that of hard-output sphere decoding [83]. Single tree search, ordered QR decomposition, channel matrix regularization, and LLR clipping are the key ingredients for realizing this type of MIMO detectors, as reference [84] mentions. With SFSD, it is necessary to perform a previous step called Successive Interference Cancellation (SIC) that needs to be performed in separated I and Q components. With 1D-NUCs, thanks to the squared shape, both components can be separated. However, this is not possible with 2D-NUCs. In this thesis, we propose an efficient preprocessing approach for 2D-NUCs based on the Voronoi regions, which has never been done in the current literature. 


\subsection{Thesis Outline and Contributions}

This thesis is divided in six chapters and two appendices. The methodology approach of the investigations is partially covered in each of the chapters of the dissertation. The reader should refer to Section 1.7 for a complete reference of the publications originated from the work carried out in the thesis. Chapter 2 explains the fundamentals of the technologies employed, which do not represent an original contribution of this dissertation. Chapters 3, 4 and 5 are the core chapters of this thesis, where key contributions are explained in detail. Chapter 6 presents the main conclusions of this dissertation, and provides additional recommendations about the use of these constellations.

Appendix A describes the physical layer simulator implemented to evaluate and assess the objectives considered in this thesis. It is based on the ATSC 3.0 specification, and has been validated during the standardization process. Both the transmitter and the receiver are explained in detail. Specific differences and commonalities between SISO and MIMO, for each of the trasnmit/receive blocks are described. Channel models used are also explained. This appendix provides the definition of some basic channels widely used in broadcast systems, and then proceeds with channel models extracted from measurement campaigns. Appendix B presents the NUC optimization algorithm employed and illustrates its application with some examples.

The key contributions of the core chapters of this thesis are the following:

\section{Chapter 3: Optimization and Performance Evaluation of Non-Uniform Constellations}

This chapter first investigates the optimization of non-uniform constellations. This thesis designs 1D-NUC constellations with modulation orders until 4096 symbols. In order to perform 1D-demapping in each component, the symmetry of the constellations is kept. Quadrant symmetric 2D-NUCs are also optimized in this chapter. They are designed with modulation orders until 256 symbols, as done in the ATSC 3.0 specification, improving the SNR performance of 1DNUCs. Two different channel models are considered: AWGN and independent and identically distributed (i.i.d.) Rayleigh. Another main contribution of this chapter is the design and implementation of a new algorithm to optimize a socalled Non-Uniform Rotated Constellation (NURC). The algorithm introduces the rotation angle as a new variable and further optimizes the capacity gain, since constellation symbols are optimized without capacity loss because of the rotation. In ATSC 3.0, NURCs were barely studied, since the use of multiRF techniques was not considered. In this thesis, the designed NURCs are also combined with these technologies, using either 2 or $4 \mathrm{RF}$ channels, and 
observing the performance gain compared to non-rotated NUCs and single-RF transmissions. These results represent the first studies of NURCs with multi$\mathrm{RF}$ techniques published in the literature. Publication [J3], listed in Section 1.7 , is derived from this chapter.

\section{Chapter 4: Low-Complexity Demapping and Quantization Algo- rithms}

Two main contributions are collected in this chapter. The first part introduces a generic demapping algorithm that reduces the number of operations to compute. The proposed demapper can be extrapolated to any constellation order. The chapter focuses on $256 \mathrm{NUC}$ constellations, which represent the highest modulation order of 2D-NUC, i.e. the most complex case, considered in this dissertation and in the ATSC 3.0 specification. The proposed algorithm is based on two different strategies. The first one takes advantage of the symmetry that constellations provide, selecting a cluster of points to compute the LLR. The second strategy exploits the condensation of NUCs, especially at low SNRs where some of the constellation symbols almost repeat the same position in the I/Q plane. Both strategies can be combined in order to reduce the number of required distances with almost no performance loss compared to the optimum ML demapper. Moreover, the proposed demapper can be combined with RC without any additional complexity. Publication [J2], listed in Section 1.7, is derived from this section. It is the first manuscript analyzing the complexity of NUCs, and some publications have been derived from this work.

The second contribution of this chapter presents an optimization method for uniform and non-uniform quantization of I/Q symbols and LLR values when using NUCs. Quantization has never been studied when using NUCs, to the best of the authors' knowledge. Although the method is applied to NUCs, can be employed using any type of constellation. The number of bits to quantize is reduced to the extent possible, while maintaining a good SNR performance. This chapter evaluates the optimized reproduction values for I/Q symbols and LLR, and investigates their influence on NUC, compared to uniform QAM constellations, in a mobile reception scenario. Publication [J5], listed in Section 1.7, is derived from this section.

\section{Chapter 5: Non-Uniform Constellations for MIMO Communications}

Chapter 5 comprehends two different contributions. First, low-order 1D-NUCs and $2 \mathrm{D}$-NUCs are optimized for $2 \times 2 \mathrm{MIMO}$ systems. The concrete parameters that affect the optimization process by the use of two transmit and receive antennas are investigated. In particular, this chapter analyzes the optimization of NUCs in one or two antennas, the influence of different demappers, and the 
use of several channel models. It also analyzes whether NUCs optimized for SISO are also optimum for MIMO, which was not considered in the ATSC 3.0 standardization process. It also provides new re-optimized constellations for multi-antenna systems and high power imbalances, which is shown to be the MIMO parameter that affects to a greater extent the NUC optimization.

The second contribution is a complete analysis from the demapping complexity point of view. The objective is to observe the difference when using NUCs instead conventional QAM constellations, and provide some guidelines for an efficient use of these constellations in MIMO. An additional solution for 2D-NUCs and fixed sphere decoders is also proposed, in order to reduce the complexity burden while keeping a good performance compared to the optimum ML demapper. Publications [J1] and [J4], listed in Section 1.7, are derived from this chapter. 


\subsection{List of Publications}

\subsubsection{Publications and Activities Related to this Thesis}

\section{International Journals}

[J1] D. Gómez-Barquero, D. Vargas, M. Fuentes, P. Klenner, S. Moon, J. Y. Choi, D. Schneider, and K. Murayama, "MIMO for ATSC 3.0," IEEE Trans. Broadcast., vol. 62, no. 1, pp. 298-305, March 2016.

[J2] M. Fuentes, D. Vargas, and D. Gómez-Barquero, "Low-Complexity Demapping Algorithm for Two-Dimensional Non-Uniform Constellations," IEEE Trans. Broadcast., vol. 62, no. 2, pp. 375-383, June 2016.

[J3] M. Fuentes, J. J. Giménez, and D. Gómez-Barquero, "Optimization and Performance of Non-Uniform Rotated Constellations with Multi-RF Transmission Techniques," IEEE Trans. Broadcast., vol. 62, no. 4, pp. 855-863, Dec. 2016.

[J4] C. Barjau, M. Fuentes, T. Shitomi, and D. Gómez-Barquero, "MIMO Solution for Successive Interference Cancellation and Two-Dimensional Non-Uniform Constellations," IEEE Communications Letters, Jan. 2017.

[J5] M. Fuentes, M. Meidlinger, D. Gómez-Barquero, and G. Matz, "Receiver Quantization in Wireless Broadcasting Systems with Non-Uniform Constellations," submitted to the IEEE Trans. Broadcast., 2017.

\section{International Research Visits}

[V1] Technische Universität Wien - Institute of Telecommunications, Vienna (Austria). February - April 2016 (3 months).

\section{Standardization Activities}

[S1] 9 contributions to the TG3/S32-2 Ad-hoc group on Modulation and Coding in the standardization process of ATSC 3.0:

- Performance evaluation and validation of BICM components: LDPC codes, bit-interleavers and NUCs.

- Evaluation of rotated NUCs for the baseline profile.

- Modulation and coding rate reduction.

- Transmit diversity techniques for MISO systems.

- Performance evaluation and optimization of MIMO precoders. 


\section{Research Projects}

[P1] Non-Uniform Constellations for Next-Generation Digital Terrestrial Broadcast System ATSC 3.0 (SP20140540).

- Funding institution: Universitat Politecnica de Valencia, Spain.

- Start date: 01/01/2015

- Duration: 12 months

[P2] Tecnologías de Radiodifusión Digital Extra-Terrestres (ET-Broadcast) (TEC2014-56483-R-AR).

- Funding institution: Ministerio de Economiía, Industria y Competividad, Spain. Co-funded by the European FEDER found.

- Start date: 01/01/2015

- Duration: 36 months

[P3] The Information Bottleneck Principle in Multiterminal Communications and Inference (TINCOIN) (ICT12-54).

- Funding institution: Wiener Wissenschafts-, Forschungs- und Technologiefonds (WWTF), Austria.

- Start date: 01/06/2014

- Duration: 24 months

\subsubsection{Other Publications}

\section{International Journals}

[J6] M. Fuentes, C. García-Pardo, E. Garro, D. Gómez-Barquero, and N. Cardona, "Coexistence of Digital Terrestrial Television and Next Generation Cellular Networks in the $700 \mathrm{MHz}$ Band," IEEE Wireless Communications, vol. 21, no. 6, pp. 63-69, Dec. 2014.

[J7] J. Ribadeneira, C. García-Pardo, M. Fuentes, D. Gómez-Barquero, and N. Cardona, "Interference Analysis for DVB-T2 Network Planning in Colombia with other Television Broadcasting Technologies," IEEE Latin America Transactions, vol. 14, no. 3, pp. 1162-1168, March 2016. 


\section{International Conferences}

[C1] C. García-Pardo, M. Fuentes, D. Gómez-Barquero, E. Garro, and N. Cardona, "Coexistence of Broadcast and Mobile Technologies in UHF bands," 23rd Session European Conference on Networks and Communications, Bologna, Italy, May 2014.

\section{COST Technical Documents}

[TD1] M. Fuentes, E. Garro, C. García-Pardo, D. Gómez-Barquero, and N. Cardona, "Coexistence of LTE and DVB Technologies in the Digital Dividend Band. A contribution to Transmission Parameters Optimization," 9th MC and Scientific Meeting, European COST IC1004 action, Ferrara, Italy, Feb. 2014.

[TD2] M. Fuentes, E. Garro, C. García-Pardo, D. Gómez-Barquero, and N. Cardona, "Coexistence of LTE and DTT Services in the First and Second Digital Dividends. Planning Studies and Potential Approaches," 10th $M C$ and Scientific Meeting, European COST IC1004 action, Aalborg, Denmark, May 2014.

[TD3] G. Martínez, M. Fuentes, C. García-Pardo, and N. Cardona, "Spectrum Sharing for LTE and DTT: Indoor LTE-A Femtocell in DVB-T2 service area," 12th MC and Scientific Meeting, European COST IC1004 action, Dublin, Ireland, Jan. 2015.

\section{National Journals}

[NJ1] D. Gómez-Barquero J. López-Sánchez, G. Martínez, J. Ribadeneira, E. Garro, C. García-Pardo, M. Fuentes, and N. Cardona, "Frequency and Network Planning and Optimization of the Digital Terrestrial Television DVB-T2 Networks in Colombia," Waves, year 6, pp. 5-19, Sept. 2014. 


\section{Chapter 2}

\section{Background}

This chapter presents the fundamental ideas and concepts on broadcasting communications utilized in this dissertation. The theoretical definitions presented in following sections constitute the basis of Chapters 3, 4 and 5, and permit to introduce minutely the different contributions of this work. Section 2.1 provides a general system overview and describes the different transmit to receive components used. The basics of BICM systems are also explained in detail, providing a general description of the three parts in which these systems are divided: FEC, BIL and modulation. This section also presents the fundamentals of MIMO systems. This includes some implementation aspects, the different gains obtained and the channel matrix description. Section 2.2 introduces the BICM capacity limits, for both SISO and MIMO systems. Implications on wireless communication systems using uniform QAM constellations are here identified. The last part of this chapter is devoted to the receiver side. In Section 2.3, the optimum ML demapper is first presented. This section also explains the suboptimum demappers that reduce complexity at the expense of some performance loss. This chapter approaches an additional feature on practical receiver implementations, i.e. quantization of the received signal. Finally, Section 2.4 extends the work to MIMO receivers.

\subsection{System Model Overview}

In DTT systems, the source of information (typically video or audio) is digitally transformed into bits and fed to the transmitter. Digital signals are adapted to the channel so the transmission mode selected maximizes to the extent possible the capacity-coverage trade-off of the system. From reference [27], one can assume that a continuous time signal $s(t)$ is transmitted over a channel $h(t)$. 


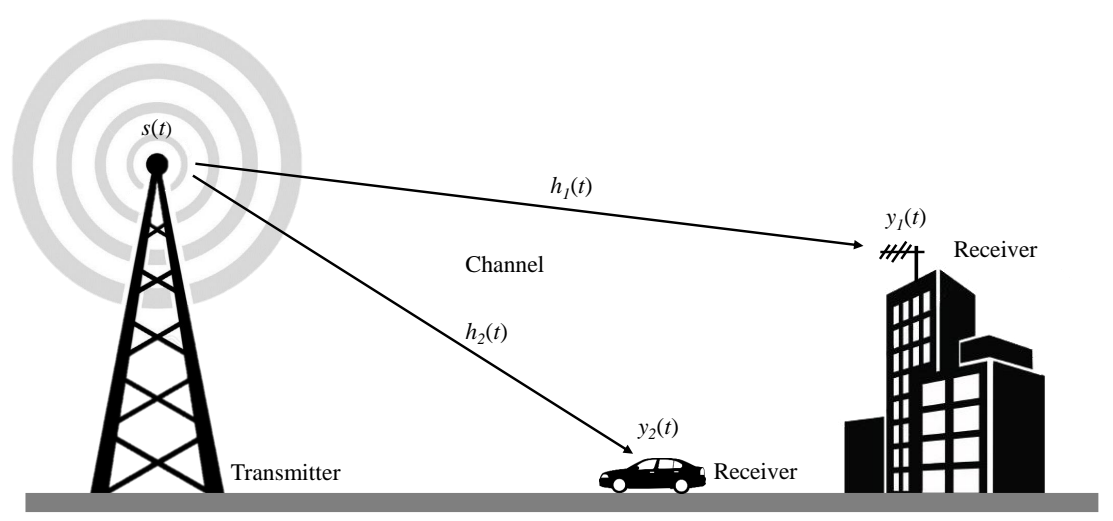

Figure 2.1: Simplified broadcasting communication system model.

As Fig. 2.1 shows, the channel distorts the signal characteristics producing a signal $y(t)$, which is different for each possible receiver. The received signal $y(t)$ is calculated as follows:

$$
y(t)=h(t) * s(t)+w(t)
$$

where $w(t)$ represents a white Gaussian noise and the operator $*$ denotes the convolution. Note that in broadcasting transmissions, there is a lack of feedback from users to the transmitter. Systems do no permit an adaptative configuration that maximizes the capacity of each individual user, and different channel realizations are obtained at the different network locations. Therefore, systems have to be configured considering the worst channel conditions and signal received. As main advantage, there is no limit in the number of users that can receive the service provided.

In digital communications, the information is transmitted every $T$ seconds, which implies an information rate of $1 / T \mathrm{~Hz}$ proportional to the signal bandwidth. To retrieve the transmitted information, receivers need to sample the signal $y(t)$ at every $0, T, 2 T, \ldots$. Assuming that the Nyquist rate criterion is fulfilled [85], the digital received signal can be represented by the discrete time baseband model, shown in Eq. 2.2.

$$
y[n]=h[n] s[n]+w[n]
$$

where $n$ denotes the $n$th sample. The described system can be easily modeled using a basic communication system block diagram, as presented in Fig. 2.2. From the sampled digital signal $y[n]$, the receiver may have to correct part of the information, corrupted due to the $h[n]$ and $w[n]$ terms. 


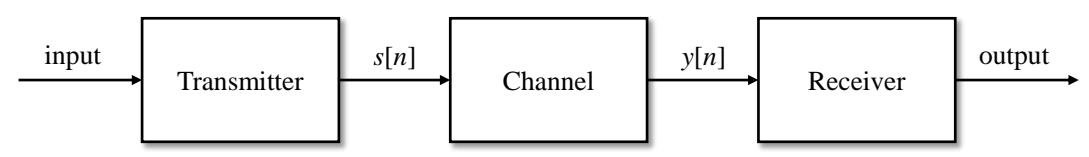

Figure 2.2: Simplified communication system block diagram.

\subsubsection{Multi-Antenna Considerations}

When $N_{T}$ transmit and $N_{R}$ receive antennas are used in a multi-antenna system, the discrete received signal is modeled as follows:

$$
\mathbf{y}[n]=\mathbf{H}[n] \mathbf{s}[n]+\mathbf{w}[n]
$$

where $\mathbf{s}$ is the $N_{T} \times 1$ transmitted vector, $\mathbf{y}$ is the $N_{R} \times 1$ received vector, $\mathbf{H}$ is the channel matrix, and $\mathbf{w}$ is the $N_{R} \times 1$ additive circularly symmetric complex Gaussian noise. In particular, $\mathbf{H}$ is a $N_{T} \times N_{R}$ matrix, with the expression:

$$
\mathbf{H}=\left[\begin{array}{cccc}
h_{11} & h_{12} & \ldots & h_{1 N_{T}} \\
h_{21} & h_{22} & \ldots & h_{2 N_{T}} \\
\vdots & \vdots & \ddots & \vdots \\
h_{N_{R} 1} & h_{N_{R} 2} & \ldots & h_{N_{R} N_{T}}
\end{array}\right]
$$

where, for instance, the index $h_{12}$ refers to the channel between first receive antenna and second transmit antenna. This is also shown in Fig. 2.3. Note that all $h$ indexes are distributed as $\mathcal{C N}(\mathbf{0}, 1)$. From now, the variable $n$ is omitted for simplicity.

In this dissertation, only $2 \times 2$ MIMO systems are evaluated, i.e. two receive and transmit antennas are considered. This implies a transmitted vector $\mathbf{s}$ with $2 \times 1$ elements, a received $\mathbf{y}$ with $2 \times 1$ elements, and $\mathbf{H}$ adopting the following form:

$$
\mathbf{H}=\left[\begin{array}{ll}
h_{11} & h_{12} \\
h_{21} & h_{22}
\end{array}\right]
$$

Note that the channel power is normalized using the squared Frobenius matrix norm, as shown in Eq. 2.6.

$$
\|\mathbf{H}\|_{F}^{2}=\sum_{i, j}^{2,2}\left|h_{i, j}\right|^{2}=\left|h_{1,1}\right|^{2}+\left|h_{1,2}\right|^{2}+\left|h_{2,1}\right|^{2}+\left|h_{2,2}\right|^{2}
$$

Moreover, indexes in each receive antenna are normalized to one, as shown in Eq. 2.7. 


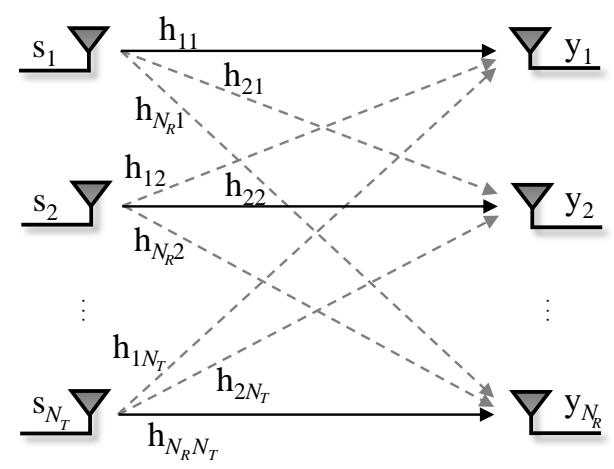

Figure 2.3: $N_{T} \times N_{R}$ MIMO channel diagram.

$$
\sum_{i}^{2}\left|h_{i, j}\right|^{2}=1
$$

With this expression, the total channel power $\|\mathbf{H}\|_{F}^{2}$ is equal to 2 , which is valid for the $2 \times 2$ MIMO scheme considered in this thesis. The matrix $\mathbf{H}$ considers that one transmit/receive antenna has vertical polarization and the other transmit/receive antenna has horizontal polarization, or vice-versa. Power Imbalance (PI) between the two transmit antennas can be also introduced. The matrix $\mathbf{H}$ can be multiplied by two additional factors, obtaining a modified channel model $\tilde{\mathbf{H}}$ :

$$
\tilde{\mathbf{H}}=\mathbf{X} \odot \mathbf{P} \odot \mathbf{H}
$$

where $\mathbf{X}$ is the Cross-Polar Discrimination (XPD) factor, $\mathbf{P}$ is the PI between antennas, $\mathbf{H}$ is the channel model matrix, and $\odot$ represents the Hadamard element-wise multiplication. The cross-polarization matrix, represents the average power ratio of the direct co-polar term that is introduced in the crosspolar term. For the case under study with two transmit and receive antennas, the matrix $\mathbf{X}$ is calculated as:

$$
\mathbf{X}=\left[\begin{array}{cc}
1 & \frac{\left|h_{12}\right|^{2}}{\left|h_{11}\right|^{2}} \\
\frac{\left|h_{21}\right|^{2}}{\left|h_{22}\right|^{2}} & 1
\end{array}\right]
$$

In this case, $\mathrm{XPD}=1 / X=\left|h_{11}\right|^{2} /\left|h_{21}\right|^{2}=\left|h_{22}\right|^{2} /\left|h_{12}\right|^{2}$. High or low XPD values represent low or high coupling between polarizations, respectively. For the extreme case with full coupling, the XPD value is 1 , while for no coupling 
$\mathrm{XPD}=\infty$. On the other hand, the power imbalance introduced between the two transmitting antennas can be modeled with the following diagonal matrix:

$$
\mathbf{P}=\left[\begin{array}{cc}
\sqrt{\beta} & 0 \\
0 & \sqrt{1-\beta}
\end{array}\right]
$$

The matrix $\mathbf{P}$ varies the power allocated at each transmit antenna according to the parameter $\beta \in[0,1]$. PI is included as part of the channel to provide a generic approach that takes into account either intentional imbalances introduced at the transmitter, or imbalances produced as part of the channel. The last element of $\mathbf{H}$ is the matrix $\tilde{\mathbf{H}}$, which depends on the channel model used, and is explained in Appendix A. Logically, the use of a single antenna in both transmitter and receiver drastically simplifies the channel model under study, since no cross-polarization discrimination and power imbalance terms are considered and therefore $h=\tilde{h}$.

\subsubsection{BICM Components}

BICM is the state-of-the-art pragmatic approach for combining channel coding with digital modulations in fading transmissions [23]. In BICM systems, the modulation constellation can be chosen independently of the CR. The structure of the BICM block consists of the serial concatenation of a FEC code, a bitinterleaver and a modulation mapper. It is one of the most important modules in a broadcast physical layer, since it provides error correction capabilities to the system, allowing the transmitter multiple choices to trade-off robustness for capacity. As explained in [34], the improvements achieved in this module represent one of the major reasons for improvements in efficiency, which is a key performance indicator to make an efficient use of the scarce radio spectrum. Fig. 2.4 presents a system block diagram based on the BICM architecture. In the following, details and a description of the functionalities and structure of each block in the chain is provided. For a complete transmit to receive block diagram, readers can refer to Appendix A.

\section{FEC Coding}

The input to the channel encoder is a bit stream $b$ with data size $N_{b}$. The channel encoder adds redundant bits, in order to increase the signal against errors generated by the channel. The encoder generates $N_{c}$ coded bits, from the $N_{b}$ original ones $\left(N_{c}>N_{b}\right)$, so the information part is contained within the codeword. The ratio $N_{b} / N_{c}$ represents the coding rate. For instance, $1 / 3$ means that just a third part of the codeword contains source information, while the rest contains parity data. In this dissertation, coding techniques based on 


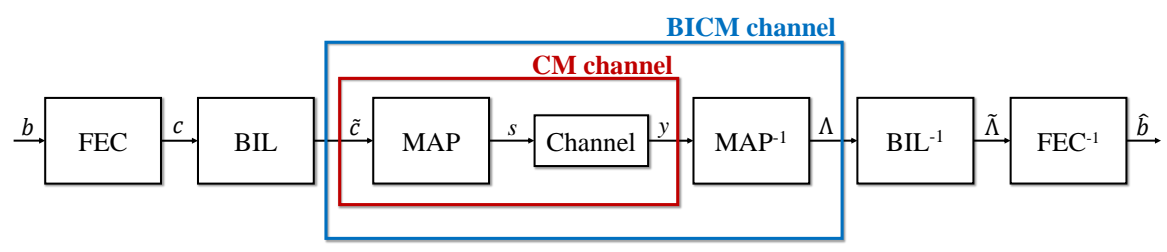

Figure 2.4: Transmit to receive BICM system block diagram.

ATSC 3.0 are used. Concretely, we use the serial concatenation of an outer $\mathrm{BCH}$ code and a inner LDPC code. Two different LDPC code lengths are defined, i.e. $L=16200$ bits (short codes) and $L=64800$ bits (long codes), with 12 possible coding rates to use, from $2 / 15$ to $13 / 15$, with step $1 / 15$.

\section{Bit-Interleaving}

The encoded bits $c$ are then bit-interleaved by using a permutation sequence that has been previously loaded, obtaining $\tilde{c}$ with size $N_{c}$. It is used to break the dependencies introduced by the channel encoder, increasing the robustness of the current transmission, i.e. improving the performance. As a drawback, BIL affects the hardware implementation. In this dissertation, a 3-stage BIL is used, which has been defined in ATSC 3.0 [15] and DVB-NGH [14] specifications. This BIL consists of a parity interleaver, a group-wise interleaver and a block interleaver. The role of the parity interleaver is to convert the staircase structure of the parity-part of the LDPC parity-check matrix into a quasi-cyclic structure similar to the information-part of the matrix enabling parallel decoding. The group-wise interleaving allows optimizing the combination between the FEC code and the constellation, and hence it is different for each constellation and CR. The block interleaver finally provides the allocation from bits to constellation symbols.

\section{Mapping}

Finally, the bit-interleaved bits $\tilde{c}$ are mapped in groups to constellation symbols s. As Fig. 2.4 shows, groups of $m$ bits are mapped to complex symbols via a one-to-one mapping function $\mu \triangleq 0,1 \rightarrow \chi$, where $\chi$ is the set of possible constellation symbols with cardinality $|\chi|=2^{m}$. The optimum shape of the constellation, i.e. how the symbols are located along the I/Q plane, depends directly on the channel, the modulation order and the coding rate, and represents the core research area of this dissertation. 


\section{Retrieving Information at the Receiver}

The received signal vector $y$ is sent to the demapper, which provides soft information about the transmitted information bits $\tilde{c}$ in the form of log-likelihood ratios $\Lambda$. The stream $\Lambda$ is de-interleaved, obtaining $\tilde{\Lambda}$. Then, it is passed to the channel decoder, which outputs an estimate vector $\hat{b}$ of the transmitted bits $b$. In BICM, demodulation and decoding are separated into independent processing blocks, thanks to the use of a bit-wise interleaving with feasible computational complexity. Solving both tasks separately introduces an information loss because bit interdependencies introduced by the channel code are no longer exploited.

\subsection{BICM Capacity Limits}

This section first introduces the Shannon theory, and then explains the capacity limits applicable when sending data modulated in the form of QAM constellations. Expressions obtained in the corresponding subsections are afterwards extrapolated to MIMO.

\subsubsection{The Unconstrained Shannon Limit}

From Eq. 2.2, a channel only perturbed by AWGN noise at the $n$th sample can be simplified to $h[n]=1$. In this case, the received signal $y$ can be expressed as follows:

$$
y[n]=s[n]+w[n]
$$

where $w[n] \sim \mathcal{C N}\left(0, \sigma^{2}\right)$ is additive circularly symmetric complex Gaussian noise, and $\sigma^{2}$ is the noise power. this expression represents a simple and straightforward model that can be used in a wide group of communication channels. The capacity (bit/s) of a AWGN channel of band $W(\mathrm{~Hz})$, whose average power is $N$, where a transmitted signal with an average power $P$ is transmitted, is given by Eq. 2.12.

$$
C(\mathrm{bit} / \mathrm{s})=W \cdot \log _{2} \frac{P+\sigma^{2}}{\sigma^{2}}
$$

This expression can be further simplified to relate normalized capacity, calculated as $C / W$, to SNR.

$$
C(\mathrm{bit} / \mathrm{s} / \mathrm{Hz})=\log _{2}(1+S N R)
$$




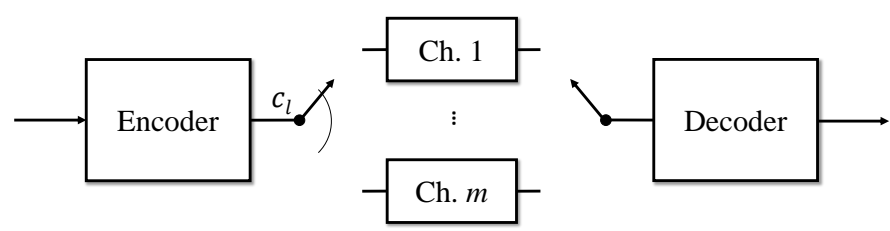

Figure 2.5: Parallel channel model for a BICM transmission.

where $S N R$ is the signal-to-noise ratio in linear units, also expressed as $P / \sigma^{2}$, with $P=|s|^{2}$ being the variance of the transmitted signal. This model is directly not applicable to practical environments. It is just an upper limit, applicable to an idealized scenario [37].

\subsubsection{Capacity Calculation for BICM}

This subsection presents the mathematical expressions for the CM and BICM capacities that denote the maximum rates achievable for a particular channel model. Let $x$ denote the transmitted signal and $y$ the received signal. Given a communication system with channel $h$, the received signal is calculated as $y=h x$. From reference [22], BICM systems can be modeled as a set of $m$ parallel binary-input channels, which are connected to the encoder output by a switch modeling an ideal interleaver, as Fig. 2.5 depicts. Each channel $m$ corresponds to a position in the signal label of the constellation $\chi$. The channel capacity is calculated as the maximum achievable rate under the constraint of uniform input distribution, and is given by Eq. 2.14 [20].

$$
C_{\mathrm{CM}}=I(x ; y \mid h)=m-E_{x, y, h}\left[\log _{2} \frac{\sum_{x^{\prime} \in \chi} p\left(y \mid x^{\prime}, h\right)}{p(y \mid x, h)}\right]
$$

where $E_{x, y, h}$ denotes expectation with respect to $x, y$ and $h, \chi$ is the set of possible constellation symbols of the transmitted signal $x$, and $m=\log _{2}(M)$, with $M$ being the number of symbols per constellation. The BICM capacity formula can be derived from Eq. 2.14. After some modifications, and considering $m$ parallel independent channels, the BICM achievable rate can be calculated as:

$$
C_{\mathrm{BICM}}=\sum_{l=1}^{m} I\left(c_{l} ; y \mid h\right)=m-\sum_{l=1}^{m} E_{x, y, h}\left[\log _{2} \frac{\sum_{x^{\prime} \in \chi} p\left(y \mid x^{\prime}, h\right)}{\sum_{x^{\prime} \in \chi_{l}^{b}} p\left(y \mid x^{\prime}, h\right)}\right]
$$


where $b \in\{0,1\}$ is equiprobable, and $\chi_{l}^{b}$ denotes the set of symbols in the constellation $\chi$ for which the code bit $c_{l}$ equals $b$. The conditional probability density function (pdf) $p(y \mid x, h)$ depends on the demapper used. Demappers are described in Section 2.3.1. This expression holds in general for all signal sets $\chi$, and for all memoryless vector channels such as AWGN, Rice or Rayleigh. It should be noted that the CM capacity is the upper-bound to the BICM capacity, as shown in the following inequality [74]:

$$
C_{\mathrm{CM}} \geq C_{\mathrm{BICM}}
$$

Despite the performance loss introduced by BICM systems, significant improvements can be obtained when using iterative decoding. As shown in [27], the BICM with iterative demapping can even reach the CM bound. However, the use of the iterative decoding is out of the scope of this dissertation.

\subsubsection{BICM Limits for Uniform QAM Constellations}

In previous calculations, no specification was provided on how to transmit constellation symbols. In this section, we follow the approach given by [38], where coded bits are mapped to the symbols using one of the many familiar ways possible: QAM. Using this type of constellations, each conventional form of MQAM has asymptotic BICM capacity at high SNRs of $\log _{2}(M)$ bit/s/Hz. Due to the limited number of different constellation symbols, the information that can be transmitted is bounded. Each successively larger QAM constellation offers a capacity increase, although it might be very small at low SNRs. However, as the author in [38] explains, there is a general trend of increasing divergence from the classic Shannon capacity at higher SNRs, even though higher orders of QAM are taken.

As an example, a Gray-coded 16QAM constellation is assumed. The Gray code is a binary system where two successive values differ in just one bit. Gray codes are used to facilitate error correction and improve the system capacity. With the considered 16QAM, 4 different coded bits are mapped to symbols, 2 to each of the independent I and Q axis. Prior to power normalization, the constellation positions on a single I/Q component are $\{-3,-1,+1,+3\}$, as Fig. 2.6 shows. Thus, symbols are mapped as $\{0,0,1,1\}$ for the MSB and $\{1,0,0,1\}$ for the LSB, in each component. Mapping the I component, bits 1 and 3 will take these values. When mapping Q, bits 2 and 4 are selected. Note that the capacity for each bit-level depends on the $\chi_{l}^{0}$ and $\chi_{l}^{1}$ alphabets, i.e. depends on the mapping chosen.

From now, it is assumed that the bits mapped to constellation symbols are independent, and that possible values are 0 or 1 . For instance, if MSB takes 0 as a value, then the transmitted symbol can be either +1 or +3 . Therefore, 


In-Phase
\begin{tabular}{|c|c|c|c|c|}
\hline Symbol position & -3 & -1 & +1 & +3 \\
\hline MSB (bit 1) & 1 & 1 & 0 & 0 \\
\hline LSB (bit 3) & 0 & 1 & 1 & 0 \\
\hline
\end{tabular}

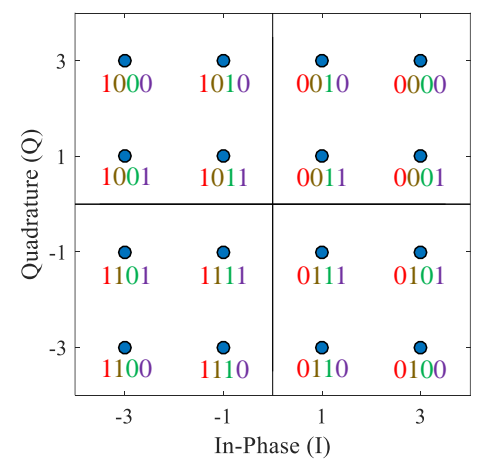

Figure 2.6: Gray mapping for 16QAM constellations.
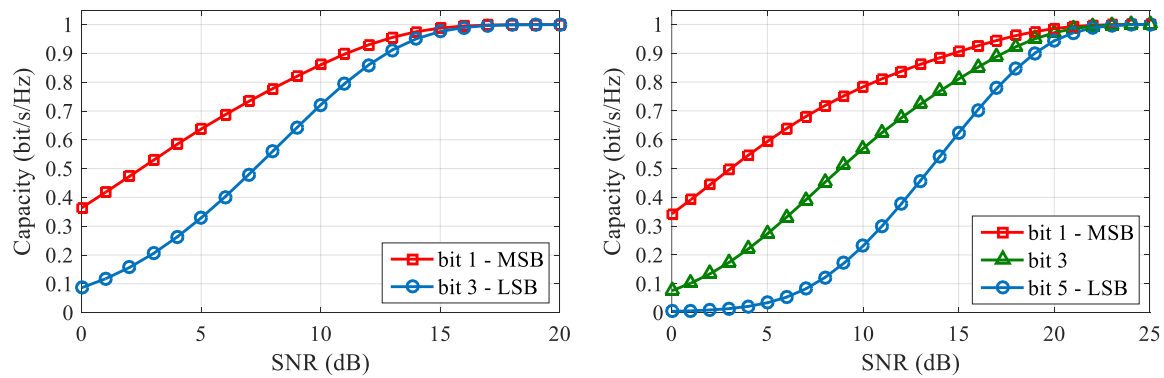

Figure 2.7: Bit-level BICM capacities in uniform 16QAM (left) and 64QAM (right) constellations, AWGN channel.

the pdf for the MSB of the received signal $p(y \mid M S B=0)$ has identical peaks at $y=+1$ and $y=+3$. The BICM capacity can be obtained for each bit level separately by applying the mutual information formula (given by Eq. 2.15) to each one. The total BICM capacity can be calculated as the sum of all bit-level capacities.

Fig. 2.7 depicts the bit-level capacities calculated for two different Graycoded 16QAM and 64QAM constellations. For the sake of simplicity, only odd bits are shown (I component). The capacity curves for even bits are identical, since QAM constellations have squared shape. As Fig. 2.7 shows, in every case the bit-level capacity reaches an asymptotic value of $1 \mathrm{bit} / \mathrm{s} / \mathrm{Hz}$, when the SNR is sufficiently high. On the other hand, MSB provides the highest capacity, and LSB tends to zero at low SNRs. This feature will be exploited by non-uniform constellations, as described in Chapter 3. 


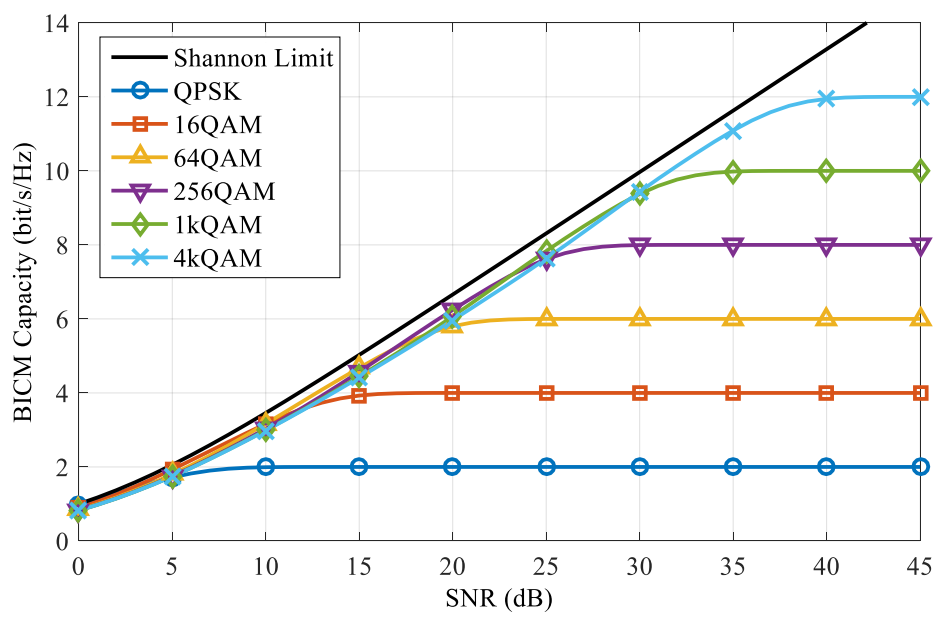

Figure 2.8: BICM capacity of uniform QAM constellations in AWGN channel.

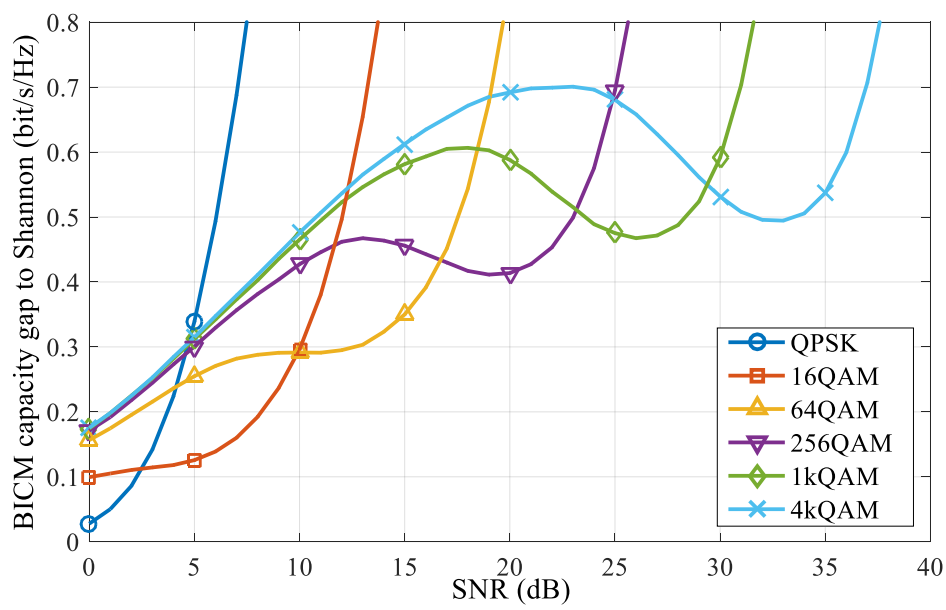

Figure 2.9: BICM capacity shrotfall of uniform QAM constellations from the Shannon theoretical limit, AWGN channel.

Fig. 2.8 depicts the total BICM capacity obtained for different orders of constellations under AWGN channel. In particular, QAM constellations with 4, $16,64,256,1024$ and 4096 symbols are shown. It is interesting to observe that the BICM limit for each constellation is not always higher than the previous one. In other words, successive QAM constellations take turns to provide the 
best BICM capacity, so depending on the SNR range in which the system works, it will be better to use a particular order of constellation. For instance, $64 \mathrm{QAM}$ is the best option around $12 \mathrm{~dB}$, while $256 \mathrm{QAM}$ works better at 18 $\mathrm{dB}$. This trend continues towards higher orders of constellation.

This is better shown plotting the shortfall of the BICM capacity from the unconstrained Shannon limit. Fig. 2.9 reveals clearly the way in which the successive QAM constellation orders take turns to provide the best capacity [38]. In this figure, it is easy to see that the capacity shortfall increases with the SNR, and also with the order of constellation. This gap to Shannon reveals that uniform distributions of QAM symbols are not optimum. Non-uniform distributions are a good solution to reduce this gap, and will be studied in next chapter.

\subsubsection{Extension to MIMO-BICM Systems}

When using multiple antennas, the channel capacity perturbed by AWGN noise can be derived from Eq. 2.13 using the Telatar expression proposed in [86]. For a channel matrix $\mathbf{H}$, the MIMO capacity when the channel coefficients are only available at the receiver, i.e. with no feedback to the transmitter, is shown in the following expression:

$$
C_{\mathrm{MIMO}}(\mathrm{bit} / \mathrm{s} / \mathrm{Hz})=\log _{2}\left(\mathbf{I}_{N_{R}}+\frac{S N R}{N_{T}} \mathbf{H H}^{\dagger}\right)
$$

The channel capacity for MIMO can be also expressed using the singular value decomposition of $\mathbf{H}$ as follows [87]:

$$
\mathbf{H}=\mathbf{U} \Sigma \mathbf{V}^{\dagger}
$$

where $\mathbf{U}$ and $\mathbf{V}$ are $N_{R} \times N_{R}$ and $N_{T} \times N_{T}$ unitary matrixes respectively, and $\Sigma$ is a rectangular matrix with size $N_{R} \times N_{T}$ and positive real numbers on the diagonal. Formula 2.18 can be used in 2.17 , which can be reformulated as:

$$
\tilde{\mathbf{y}}=\Sigma \tilde{\mathbf{x}}+\tilde{\mathbf{w}}
$$

where $\tilde{\mathbf{y}}=\mathbf{U}^{\dagger} \mathbf{y}, \tilde{\mathbf{x}}=\mathbf{V}^{\dagger} \mathbf{x}$, and $\tilde{\mathbf{w}}=\mathbf{U}^{\dagger} \mathbf{w}$. Taking into account that $\tilde{\mathbf{w}}$ is equally distributed as $\mathbf{w}$, the channel capacity can be also expressed as a function of the singular values of $\mathbf{H}$, as shown in Eq. 2.20.

$$
C_{\mathrm{MIMO}}(\mathrm{bit} / \mathrm{s} / \mathrm{Hz})=\sum_{i=1}^{N_{\min }} \log _{2}\left(1+\frac{S N R}{N_{T}} \lambda_{i}^{2}\right)
$$

where $\lambda_{i}$ is the $i$ th diagonal entry of $\Sigma$. Note that in Eq. 2.20 the channel can be divided into parallel channels. In this case, both individual channels are 
given by the singular values of the channel matrix $\mathbf{H}$. In the above expression, $n_{\min }$ is the number of non-zero values that depend on the rank of the channel matrix. The higher the value of $n_{\text {min }}$, the larger the number of parallel channels in which information can be transmitted through. Therefore, the maximum MIMO capacity can be achieved only when $\mathbf{H}$ is full rank. In other words, when columns or rows are linearly independent, i.e. when $N_{R}>N_{T}$ or $N_{T}>N_{R}$, respectively.

The coded modulation maximum achievable rate can be also extended to MIMO. In this case, the channel capacity under the constraint of uniform input distribution is given by the following expression:

$$
C_{\mathrm{CM}}^{\mathrm{MIMO}}=I(\mathbf{x} ; \mathbf{y} \mid \mathbf{H})=m-E_{\mathbf{x}, \mathbf{y}, \mathbf{H}}\left[\log _{2} \frac{\sum_{\mathbf{x}^{\prime} \in \chi} p\left(\mathbf{y} \mid \mathbf{x}^{\prime}, \mathbf{H}\right)}{p(\mathbf{y} \mid \mathbf{x}, \mathbf{H})}\right]
$$

where $E_{\mathbf{x}, \mathbf{y}, \mathbf{H}}$ denotes expectation with respect to $\mathbf{x}, \mathbf{y}$ and $\mathbf{H}$, and $\chi$ is the set of possible constellation symbols. Taking into account this expression, the BICM achievable rate can be also extrapolated to MIMO as follows:

$$
C_{\mathrm{BICM}}^{\mathrm{MIMO}}=\sum_{l=1}^{m} I\left(c_{l} ; \mathbf{y} \mid \mathbf{H}\right)=m-\sum_{l=1}^{m} E_{\mathbf{x}, \mathbf{y}, \mathbf{H}}\left[\log _{2} \frac{\sum_{\mathbf{x}^{\prime} \in \chi} p\left(\mathbf{y} \mid \mathbf{x}^{\prime}, \mathbf{H}\right)}{\sum_{\mathbf{x}^{\prime} \in \chi_{l}^{b}} p\left(\mathbf{y} \mid \mathbf{x}^{\prime}, \mathbf{H}\right)}\right]
$$

As occurred for SISO, this expression holds in general for all signal sets $\chi$, and for all memoryless vector channels such as AWGN, Rice or Rayleigh, depending on the choice of $p\left(\mathbf{y} \mid \mathbf{x}^{\prime}, \mathbf{H}\right)$. Both equations 2.21 and 2.22 are the maximum achievable rates for a $\mathrm{CM}$ and BICM communication system with $N_{T}$ and $N_{R}$ antennas, and vanishing error probability.

\subsection{Single-Antenna Receivers}

This section describes first two different demappers for demodulation. The presented demappers evaluate the distance from the received symbol $y$ to all possible constellation symbols $x$. The 1D-demapping strategy, which can be combined with both demappers, is presented afterwards. This section also introduces the basics of quantization. 

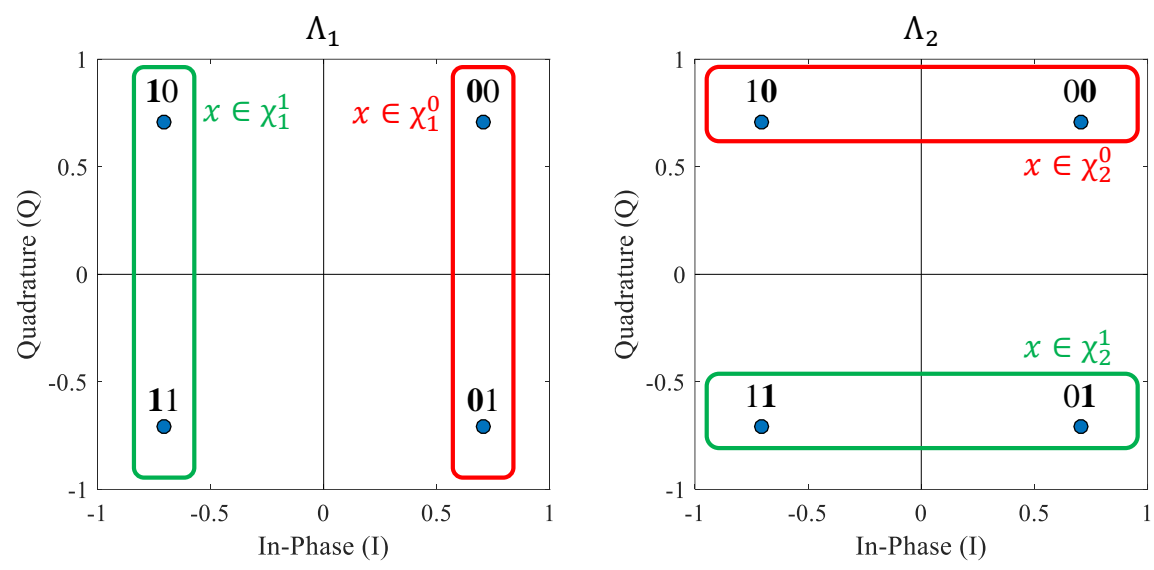

Figure 2.10: Complementary sets of transmitted symbols to compute $\Lambda_{1}$ and $\Lambda_{2}$, using a QPSK constellation.

\subsubsection{Demapping Algorithms}

\section{Optimum Maximum-Likelihood Demapper}

The optimum ML demapper minimizes the probability of error of the transmitted code bits, and it is expressed in the form of LLRs. From the received symbol vector $y$, and the channel vector $h$, each $\log$-likelihood ratio (LLR) $\Lambda_{l}$ is computed for all code bits $c_{l}, l=1, \ldots, B$, with $B$ as the number of bits that affect each dimension of a constellation. $B$ does not refer to the number of bits per symbol $(m)$. For the LLR computation, a total number of $M$ Euclidean distances between the received symbol $y$ and all constellation symbols $x$ is calculated. A single output $\Lambda_{l}$ can be computed using Eq. 2.23.

$$
\Lambda_{l} \triangleq \log \frac{p\left(c_{l}=1 \mid y, h\right)}{p\left(c_{l}=0 \mid y, h\right)}=\log \frac{\sum_{x \in \chi_{l}^{1}} p(y \mid x, h)}{\sum_{x \in \chi_{l}^{0}} p(y \mid x, h)}
$$

where $p\left(c_{l}=1 \mid y, h\right)$ is the probability function of the transmitted coded bits conditioned to the received vector $y$ and the channel matrix $h, x$ is a possible transmitted symbol, and log refers to the natural logarithm. $\chi_{l}^{1}$ and $\chi_{l}^{0}$ denote the complementary sets of transmit vector $x$ for which $c_{l}=1$ and $c_{l}=0$ respectively. The amount of complementary sets $\chi_{l}^{1}$ and $\chi_{l}^{0}$ has to be $B$. Fig. 2.10 shows an example of the complementary sets for a QPSK constellation, where two different $\Lambda_{1}$ and $\Lambda_{2}$ are computed. The optimum ML demapper 
has therefore to consider $M=2^{B}$ symbols to previously calculate all possible distances, and then calculate the LLRs. The conditional $p(y \mid x, h)$ is given by the following expression:

$$
p(y \mid x, h)=\frac{1}{\left(\pi \sigma^{2}\right)} \exp \left(\frac{-|y-h x|^{2}}{\sigma^{2}}\right)
$$

where $\sigma^{2}$ represents the noise variance. From Eq. 2.24, and after some expression manipulations [28], each LLR $\Lambda_{l}$ can be computed as:

$$
\Lambda_{l}=\log \frac{\sum_{x \in \chi_{l}^{1}} \exp \left(-\frac{|y-h x|^{2}}{\sigma^{2}}\right)}{\sum_{x \in \chi_{l}^{0}} \exp \left(-\frac{|y-h x|^{2}}{\sigma^{2}}\right)}
$$

With ML, the complexity increases exponentially with the constellation order. For each demapping execution the complexity order is $\mathcal{O}(M)$, where $M$ is the cardinality of the symbol constellation. This complexity is usually considered too high when using real receivers, which have to compute a huge number of distances in real-time transmissions. In that sense, suboptimum demappers may are useful to reduce complexity in the sense of distances to compute, while keeping a good performance. In Chapter 4, different suboptimum demappers are proposed.

\section{Max-log Demapper}

The max-log approximation is one of the most common algorithms used to reduce the ML demapping complexity. Max-log demappers substitute the logarithm and exponential functions using the following approximation:

$$
\log \left(\sum_{i} \exp \left(x_{i}\right)\right) \approx \max _{i} x_{i}
$$

From this expression, the demapping process is reduced to a minimum distance problem. Applying the max-log approximation to Eq. 2.25, each LLR $\Lambda_{l}$ can be computed with max-log as:

$$
\Lambda_{l}=\min _{x \in \chi_{l}^{0}}\left(\frac{|y-h x|^{2}}{\sigma^{2}}\right)-\min _{x \in \chi_{l}^{1}}\left(\frac{|y-h x|^{2}}{\sigma^{2}}\right)
$$

Although the amount of calculations is reduced, the complexity burden for each execution of max-log demappers has still order $\mathcal{O}(M)$, which scales linearly with the number of demodulation executions, as ML does. As demonstrated in 
[88], the use of max-log entails a performance loss compared to ML that depends on the SNR. Note that both ML and max-log demappers can be combined with 1D-demapping, reducing the complexity order down to $\mathcal{O}(\sqrt{M})$. The use of this demapper is explained in the following section.

\section{D-demapping}

Prior to expressions 2.25 (ML) and 2.27 (max-log), the demapper has to consider $2^{B}$ symbols in a two-dimensional space, calculating all $M$ distances from the received symbol $y$ to all possible constellation symbols $x$. However, this calculation can be simplified by just calculating the distances in both components individually.

As reference [26] proposes, it is possible to use the mapping feature that the original constellation points in the same row or column belong to the same subset for a specific even or odd bit (i.e., the subset with the bit value $c_{l}$ being 0 or 1). The idea is to select the best candidate in each row and column, finding the minimum Euclidean distance just in one dimension. In a constellation with $M$ symbols, only $\sqrt{M}$-best candidates or distances are needed for the LLR calculation of all even bits. The same condition applies to all odd bits. This provides significant complexity reduction, while the final result is exactly the same as searching from the full set. With 1D-demapping, the complexity can be reduced to $\mathcal{O}(\sqrt{M})$. The difficulty arises in how to quickly find the closest symbols in the faded constellation after the I and Q components are distorted by a different fading.

Taking even bits as an example of calculation, the straightforward method to find the best candidate in each column is to project the received signal to the column, and to select the closest one. Parallel columns to the received symbol can be calculated as follows:

$$
Y=-\frac{h_{Q} \cos \alpha}{h_{I} \sin \alpha} X+\left(x_{Q}+\frac{h_{Q} \cos \alpha}{h_{I} \sin \alpha} x_{I}\right)
$$

where $\left(a_{I}, a_{Q}\right)$ is the coordinate of a faded constellation symbol $a=h x$ located in the column, $\alpha$ is the angle of $h$, and $X$ and $Y$ are the straight line variables (different from the constellation symbol $x$ and the received symbol $y$ ). The perpendicular line which passes through the received signal $y$ can be expressed as:

$$
Y=-\frac{h_{I} \sin \alpha}{h_{Q} \cos \alpha} X+\left(y_{Q}+\frac{h_{I} \sin \alpha}{h_{Q} \cos \alpha} y_{I}\right)
$$




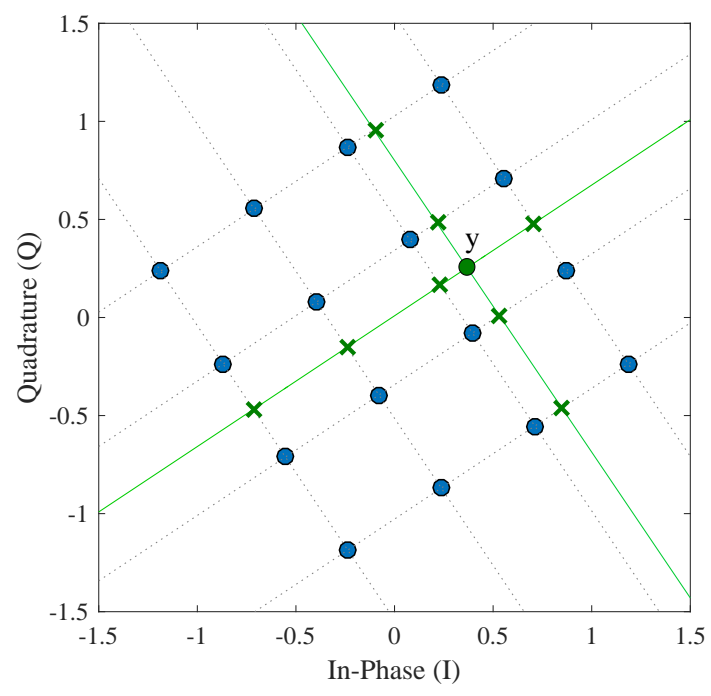

Figure 2.11: 16QAM constellation with transmitted symbols $x$, received symbol $y$ and cross points $c$ calculated.

For the sake of simplicity, $\beta_{I S}=h_{I} \sin \alpha$ and $\beta_{Q C}=h_{Q} \cos \alpha$. Then, the cross points $\left(c_{I}, c_{Q}\right)$ of the lines calculated in 2.28 and 2.29 can be calculated as:

$$
\begin{aligned}
& c_{I}=\frac{y_{I} \beta_{I S}^{2}+a_{I} \beta_{Q C}^{2}-\left(y_{Q}-a_{Q}\right)\left(\beta_{I S} \beta_{Q C}\right)}{\beta_{I S}^{2}+\beta_{Q C}^{2}} \\
& c_{Q}=\frac{y_{Q} \beta_{Q C}^{2}+a_{Q} \beta_{I S}^{2}-\left(y_{I}-a_{I}\right)\left(\beta_{I S} \beta_{Q C}\right)}{\beta_{I S}^{2}+\beta_{Q C}^{2}}
\end{aligned}
$$

Eqs. 2.30 and 2.31 are repeated for $\sqrt{M}$ symbols in each direction, obtaining the different $\sqrt{M}$ cross points as Fig. 2.11 shows. In a final step, distances $|y-h x|$ are computed, and LLRs are calculated from those distances using expressions 2.25 and 2.27, when using ML and max-log demappers respectively For the odd bits, $\beta_{I S}$ is substituted by $\beta_{I C}=h_{I} \cos \alpha$, and $\beta_{Q C}$ by $\beta_{Q S}=$ $h_{Q} \sin \alpha$, and the rest of the process is repeated.

\subsubsection{Signal Quantization}

Quantization is a basic operation that transforms continuous to discrete signals. Naturally, quantization follows a performance-complexity trade-off, that 


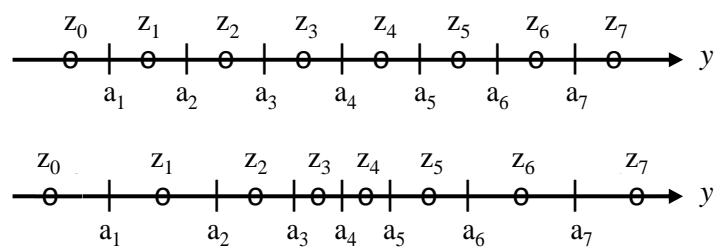

Figure 2.12: Example of uniform (top) and non-uniform (bottom) quantizers, with $a_{0}=a_{8}$ $=\infty$.

is, signal accuracy increases with the number of quantization bits. However, high resolution signals are more costly to store and process. A low quantization resolution is highly beneficial for hardware implementations since it entails a reduction of chip area, which in turn yields a lower manufacture cost and power consumption. Several processing blocks at the receiver need a certain amount of samples in order to compute the specific algorithm implemented.

In terrestrial broadcasting receivers, the highest memory requirements are always needed in the TDIL [28]. As reference [27] denotes, systems have to cope with signal strength fluctuations over time occurring during the motion of the receiver terminals. The TDIL interlaces multiple constellation symbols to increase the signal time diversity, and hence to increase the resilience against fading. The memory required to store all the received symbols of the TDIL block will depend on the number of quantization levels and their distribution. More levels imply greater fidelity but also need more memory to store. The total amount of memory needed at the TDIL, denoted in this dissertation as $\Delta$, can be calculated as expressed in Eq. 2.32.

$$
\Delta=\Gamma \times Q=\Gamma \times \sum_{l=1}^{L} q_{l}
$$

where $\Gamma$ represents the interleaving depth, $Q$ denotes the total number of bits, $q_{l}$ is the number of bits per reproducer, and $L$ is the number of elements to quantize.

A scalar quantizer can be defined as a set of intervals, $S=\left\{s_{i} ; i \in I\right\}$, where the index set $I$ is a collection of consecutive integers beginning with 0 or 1 , together with a set of reproduction values $R=\left\{z_{i} ; i \in I\right\}$ [67]. We also define $a_{i}$ as the thresholds or boundaries to define each region $i$. The set of reproduction values presented in Fig. 2.12 can be equally or non-equally spaced, considering uniform or non-uniform quantization. Note that this concept is totally different from uniform and non-uniform constellations, where the distribution refers to constellation symbols. 

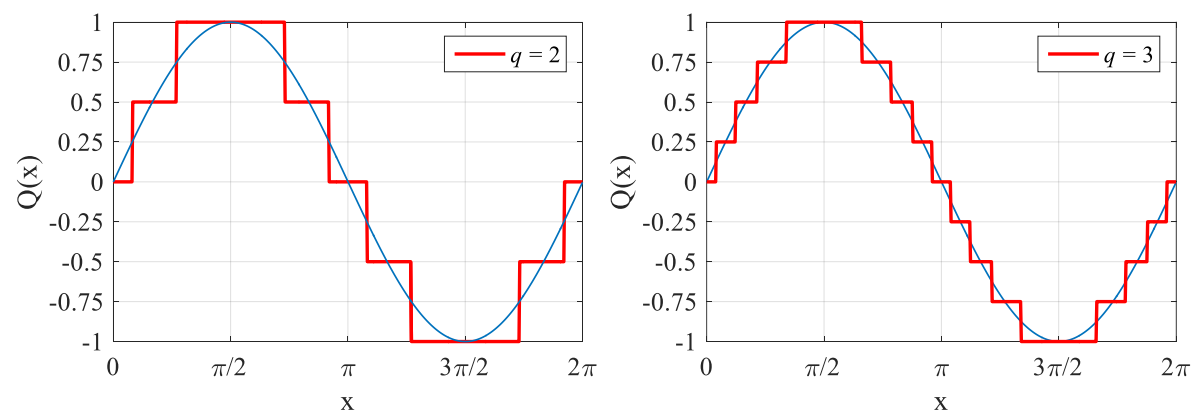

Figure 2.13: Example of uniform-quantized signals, using 2, or 3 bits, i.e. 4 and 8 possible levels.

With uniform quantization, real and imaginary parts are quantized separately and uniformly. A quantizer is said uniform when the levels $z_{i}$ are equispaced. Fig. 2.12 shows an example of uniform quantization (top). With non-uniform quantization, reproducers are no longer equispaced. The thresholds $a_{i}$ are just calculated as the middle point between two symbols. The use of non-uniform quantizers further improves the system performance, since they can adapt reliably to the signal statistics, providing more faithful results. Nonuniform quantizers may need less quantization levels, i.e. lower implementation complexity at the receiver, while keeping the same performance.

The reproducers $z_{n}$ and boundaries $a_{n}$ are designed to represent efficiently the input random variable $y$, as Fig. 2.13 depicts. In Chapter 4, optimum quantizers in the sense of maximum BICM capacity are investigated. Specific quantization values are obtained using the Nelder-Mead algorithm [89], as done for constellation optimization. This algorithm is widely described in Appendix $\mathrm{B}$, and obtains the optimum reproducers and boundaries that maximize the BICM capacity of the system when considering quantization at the TDIL. These quantizers are applied to LLR values or single I and Q components, and evaluated depending on system parameters such as number of bits, code rate and constellation order.

\subsection{Multi-Antenna Receivers}

In this section, extensions of the two different demapping algorithms presented for SISO are first provided. Although these demappers obtain optimum performance, they entail a high demapping complexity, which increases with the order of constellation and number of antennas. In this dissertation, three addi- 
tional algorithms are used to reduce complexity: ZF, Minimum Mean Square Error (MMSE) and SD demodulators, which are also described in following subsections. Please note that quantization for MIMO systems is out of the scope of this thesis.

\subsubsection{ML and Max-Log Demappers}

When using multiple antennas, the optimum ML demapper computes LLRs $\Lambda_{l}$ for all code bits $c_{l}, l=1, \ldots, B \times N_{T}$. $B$ refers to the number of bits per symbol used in a single antenna, and $N_{T}$ is the number of transmit antennas. For the LLR computation, a total number of $M^{N_{T}}$ Euclidean distances between the received vector $\mathbf{y}$ and constellation symbols $\mathbf{x}$ is calculated. A single output $\Lambda_{l}$ can be computed as:

$$
\Lambda_{l}=\log \frac{\sum_{\mathbf{x} \in \chi_{l}^{1}} \exp \left(-\frac{\|\mathbf{y}-\mathbf{H} \mathbf{x}\|^{2}}{\sigma^{2}}\right)}{\sum_{\mathbf{x} \in \chi_{l}^{0}} \exp \left(-\frac{\|\mathbf{y}-\mathbf{H} \mathbf{x}\|^{2}}{\sigma^{2}}\right)}
$$

With MIMO, the complexity increases exponentially with the constellation order and the number of antennas. Please note that in this dissertation, the case of iterative detection with a priori information is not considered. For each demapping execution the complexity order is $\mathcal{O}\left(M^{N_{T}}\right)$, where $M$ is the cardinality of the symbol constellation. The complexity is further increased when comparing with SISO, since the demapping process has to be extended to all possible $N_{R}$ antennas. In other words, every antenna has to be considered in the demapper, since every $y_{n}$ with $n=1, . ., N_{R}$ contains information about all transmitted signals $s_{t}$, with $t=1, \ldots, N_{T}$. Hence, it is not possible to perform separated demapping processes in each antenna without cancelling part of the information and loosing performance.

The same reasoning can be extended to max-log demappers, just reducing the process to a minimum distance problem. With MIMO, the LLRs can be computed with max-log as:

$$
\Lambda_{l}=\min _{\mathbf{x} \in \chi_{l}^{0}}\left(\frac{\|\mathbf{y}-\mathbf{H} \mathbf{x}\|^{2}}{\sigma^{2}}\right)-\min _{\mathbf{x} \in \chi_{l}^{1}}\left(\frac{\|\mathbf{y}-\mathbf{H} \mathbf{x}\|^{2}}{\sigma^{2}}\right)
$$

The complexity burden for each execution of max-log demappers has still order $\mathcal{O}\left(M^{N_{T}}\right)$. 


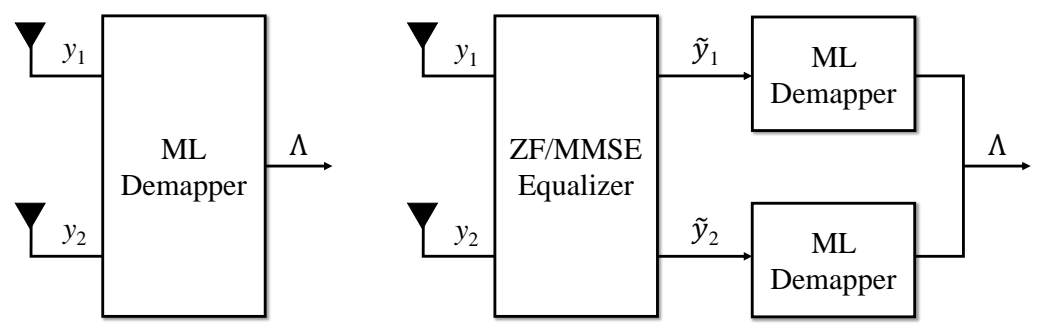

Figure 2.14: Two demapping configurations considered. Optimum ML (left) vs. ZF/MMSE (right).

\subsubsection{ZF and MMSE Detectors}

Linear demodulators represent a good alternative to the optimum MIMO demapper, since they reduce drastically the complexity while maintaining a good performance. This type of demodulators transform the joint MIMO demapping process into independent single-antenna demapping blocks. For instance, in a $2 \times 2$ MIMO system, a demapper with complexity $M^{2}$ is transformed into two independent demappers with complexity $M$. To do this, linear demodulators such as ZF or MMSE use an equalizer that suppresses the cross components of the channel $\left(h_{12}, h_{21}\right.$, etc. $)$ that act as an interference, and provides an estimate of the transmitted symbols [90]. Fig. 2.14 shows two different demappers, with and without ZF/MMSE.

The ZF detector sets the interferers amplitude to zero, which is simply done by inverting the channel response and rounding the result to the closest symbol in the constellation alphabet that is transmitted [82]. There are two ways of performing $\mathrm{ZF}$. In case $N_{R}>N_{T}$, the pseudo-inverse of the channel matrix is used, as shown in Eq. 2.35.

$$
\tilde{\mathbf{y}}=\mathcal{Q}\left\{\left(\mathbf{H}^{\dagger} \mathbf{H}\right)^{-1} \mathbf{H}^{\dagger} \mathbf{y}\right\}
$$

where $\mathcal{Q}(\cdot)$ stands for component-wise quantization, y is the received vector, and $\mathbf{H}$ is the MIMO channel matrix. The estimated transmit vector is denoted by $\tilde{\mathbf{y}}$. The matrix that pre-multiplies the received vector is often called as nulling matrix. As a second and simpler option, when the MIMO channel matrix is square, i.e $N_{R}=N_{T}$, and invertible, ZF just uses the inverse of the channel matrix, as shown in Eq. 2.36.

$$
\tilde{\mathbf{y}}=\mathcal{Q}\left\{\mathbf{H}^{-1} \mathbf{y}\right\}
$$

In this dissertation, since only $2 \times 2$ MIMO systems are considered, formula 2.36 is used. The main drawback of ZF detectors is the fact that they only 
focus on cancelling completely the channel interference, which enhances the noise. As a solution, MMSE detectors can be used since they counteract the noise enhancement problem of the ZF detectors. MMSE detectors estimate $\mathbf{y}$, minimizing the error due to the noise and the interference combined. MMSE detectors use the following estimation step [91].

$$
\tilde{\mathbf{y}}=\mathcal{Q}\left\{\left(\mathbf{H}^{\dagger} \mathbf{H}+\sigma^{2} \mathbf{I}\right)^{-1} \mathbf{H}^{\dagger} \mathbf{y}\right\}
$$

The use of MMSE equalization was firstly proposed in [92] and [93] for reducing the Inter-Symbol Interference (ISI) in communications, and afterwards applied in [94], [33] to wireless systems.

\subsubsection{Sphere Decoding Techniques}

The main idea under Sphere Decoders (SD) comes from the expression of the $N_{T}$-dimensional squared Euclidean distance in Eq. 2.33 as an addition of onedimensional squared Euclidean distances [95]. SD methods look for the ML solution, but using just a subset of the total $M^{N_{T}}$ constellation symbols. This subset is a $N_{T}$-dimensional sphere centered at the received signal vector $y$, with a certain radius $(\rho)[56],[81]$. The sphere radius constraint $\rho$ is included in the ML detection formula as in Eq. 2.38

$$
\Lambda_{l}=\log \frac{\sum_{\mathbf{x} \in \chi_{l}^{1}} \exp \left(-\frac{1}{\sigma^{2}}\left(\|\mathbf{y}-\mathbf{H x}\|^{2} \leq \rho\right)\right)}{\sum_{\mathbf{x} \in \chi_{l}^{0}} \exp \left(-\frac{1}{\sigma^{2}}\left(\|\mathbf{y}-\mathbf{H x}\|^{2} \leq \rho\right)\right)}
$$

In SD, only distances that accomplish $\|\mathbf{y}-\mathbf{H x}\| \|^{2} \leq \rho$ are considered. Fig. 2.15 depicts an example of SD with two particular radius $\rho_{1}$ and $\rho_{2}$, using a QPSK constellation with $M=4$. AWGN channel, $(\mathbf{H}=\mathbf{I})$ and a $2 \times 2$ MIMO system are assumed. In this example, depending on the sphere radius chosen, there are four or just one symbol that lie inside the sphere to calculate the ML solution. These symbols represent the candidate solutions that would fulfill Eq. 2.38. The ML solution would then be the closest lattice point of the list of candidate points to the received vector $y$. The use of $\rho_{1}$ and $\rho_{2}$ implies a complexity reduction of $93.75 \%$ and $75 \%$ respectively. Note that the complexity reduction increases with the constellation order. However, the selection of a suitable radius $\rho$ is not an easy task. Several methods have been provided to estimate the sphere radius, which can be found in [56].

In SD, a SIC detection with QR factorization of the channel matrix is employed $(\mathbf{H}=\mathbf{Q R})$. This factorization transforms the ML demapping process into an analog method that is solved through a tree structure. Once the tree 


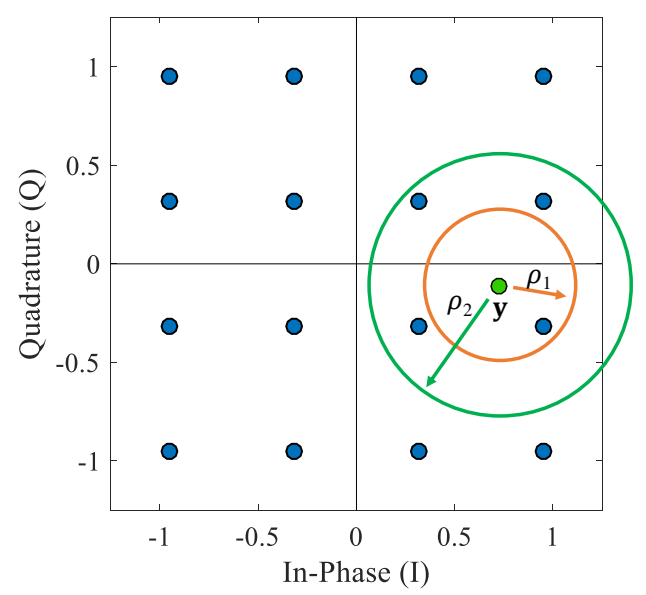

Figure 2.15: Sphere Decoding of a QPSK in a 2x2 MIMO system, with two different radius $\rho_{1}$ and $\rho_{2}$, AWGN channel. The selection of a particular radius will define the performance loss introduces by SD.

is defined, different algorithms can be used to reach the SD solution. Many tree-search strategies are proposed in the current literature. Some of them are described in references [96] and [97].

The main problem of SD methods from an implementation point of view is the variable complexity. To overcome that problem, Fixed-Complexity Sphere Decoders (FSD) can be used. As reference [82] explains, FSD combines a preprocessing stage followed by a predetermined tree-search stage. The symbols are detected following a specific ordering as proposed in [98]. The predetermined tree-search stage is divided in turn in two different phases: full expansion in the first $T$ tree levels, and single-path expansion in the remaining $N_{T}-T$ levels. It is really important to select a suitable $T$ value. In [99], the chosen value is always:

$$
T \geq \sqrt{N_{T}}-1
$$

Although FSD does not guarantee to find the ML estimate in all cases, it achieves the maximum detection diversity when choosing this value. At the full expansion phase, all possible values of the constellation are assigned to the symbol at the current level. The single-path expansion phase starts from each retained path, and continues calculating the solution of the remaining SIC problem in the tree. Note that FSD achieves quasi-ML hard detection performance. 


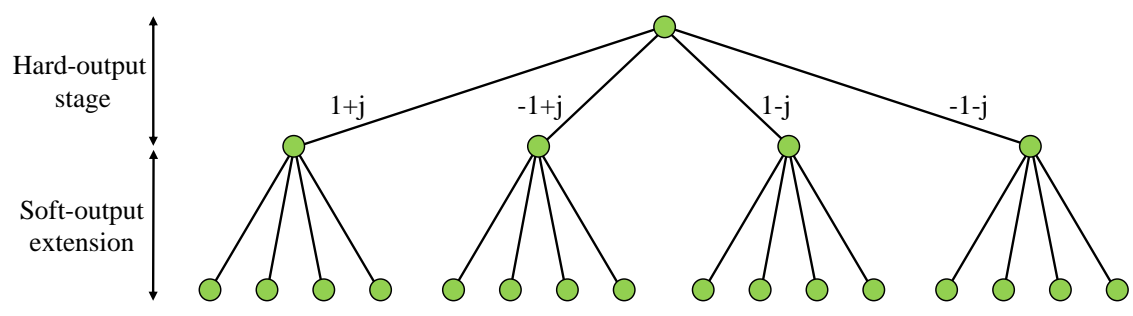

Figure 2.16: Decoding tree of the SFSD algorithm for a $2 \times 2$ MIMO system with a QPSK constellation, $N_{\text {iter }}=2, T=1$.

An interesting approach to provide soft information after the FSD search is the SFSD algorithm, proposed in [83]. As reference [82] denotes, the SFSD demapper first carries out a conventional hard-output FSD tree-search. This search gives as a result $M$ SIC branches of $M$ symbols each, from where the path with the minimum accumulated partial Euclidean distance is selected as the ML solution. In a second step, the SFSD extends the FSD tree search in order to obtain the minimum distances. The SFSD starts from the candidate list obtained in the first stage, and adds new candidates for the counter-hypotheses. Since the first level is already expanded, LLR values of symbol bits in this level are all available. To start with the list expansion, the best $N_{i t e r}$ paths are selected from the initial list. The symbols belonging to these paths are picked up from the root, up to a specific level $l$. At level $l-1$, additional $\log _{2}(M)$ branches are explored, each of them having one of the bits of the initial path symbol negated. The new partial paths are then completed using the SIC path, and the same operation is repeated until the lowest level is reached. An example of the SFSD tree search is shown in Fig. 2.16, for a $2 \times 2$ MIMO system with $N_{\text {iter }}=2, T=1$ and QPSK is the constellation used.

As main drawback, SFSD cannot be used when transmitting 2D-NUCs. The hard-output stage that provides the SIC solutions needs to be performed in separated I and Q components. With 2D-NUCs, both components cannot be separated, and a different approach is needed in this case. In Chapter 5, we propose a solution based on the Voronoi regions that solves the problem and allows SFSD with these constellations. 


\section{Chapter 3}

\section{Optimization and Performance Evaluation of Non-Uniform Constellations}

This chapter presents the main procedures and results examined in this thesis for NUC optimization. These constellations are evaluated from the transmitter point of view, for single-antenna communications. The chapter is divided into four sections. Section 3.1 investigates two optimization shaping techniques, depending on the number of real-valued dimensions considered in the optimization process: one-dimensional and two-dimensional NUCs. It also presents the improvements achieved in terms of BICM capacity. Section 3.2 provides two different methods for the optimization of rotated NUCs, and Section 3.3 explains in detail the multi-RF technique utilized in this thesis, i.e. channel bonding. Performance results and SNR gains achieved are also provided in Section 3.4. Finally, the main findings of this work are summarized in 3.5.

\subsection{Non-Uniform Constellations Optimization}

The theoretical approach given by Shannon in [11] indicates that the best capacity for an AWGN channel is only obtained if the received signal $y$ has Gaussian distribution. However, broadcast communications traditionally have used QAM constellations, which imply received signals with nearly rectangular 
distributions. As reference [38] indicates, this distribution becomes more and more rectangular as the constellation order increases. Furthermore, the use of discrete distributions in which is known as constellations clearly affects the transmitted signal itself. The main idea under non-uniform constellations is to adapt the discrete distribution, i.e. constellation symbols to the channel, adopting a Gaussian-like shape that maximizes the capacity. The study of these constellations for broadcast transmissions has been explored in several studies. Reference [38] was the first research approaching the concept of 1DNUCs, and the most important contributions that explain the use of 2D-NUCs are [17], [24] and [43].

With NUCs, the constellation symbols are optimized by means of signal shaping techniques to provide an improved performance compared to uniform QAM constellations. These constellations are designed for a particular SNR and specific channel model. NUCs make the transmitted constellation distribution more Gaussian, and the gain obtained from this change is called shaping gain. In [100], it was shown that the shaping gain introduced by discrete constellations in AWGN channels cannot exceed $10 \log (\pi e / 6) \approx 1.53 \mathrm{~dB}$, where $e$ represents the Euler's number. However, this asymptotic value only can be reached if high-order constellations are used, at high SNR values. These constellations will be the basis for the rest of the thesis. Note that NUCs are obtained for ideal systems in which estimation and hardware implementation aspects are not considered. For real transmissions, an additional performance loss is introduced, and therefore the SNR changes [28]. However, differences in constellation symbols are almost negligible within SNR ranges of 1-2 dB, as following sections show. The optimization process of both types of constellation as well as the performance gains obtained are explained in following sections.

\subsubsection{One-Dimensional Non-Uniform Constellations}

The total Degrees of Freedom (DoF) in the 1D-NUC optimization are $\frac{\sqrt{M}}{2}-1$, where $M$ represents the number of symbols of the constellation under evaluation. Optimizing higher order constellations requires to increase the number of variables, which also increments the optimization burden. The number of optimization parameters for constellations with cardinality of 16, 64, 256, 1024, and 4096 points, require: 1, 3, 7, 15 and 31 optimization variables, respectively. In general, the optimization starts from Gray mapping and calculates the constellation symbols on one axis from the parameters indicated in Table 3.1 .

Following the approach given by [38], the aim is to identify the optimum parameters $\mathbf{a}=\left[a_{0}, a_{1}, \ldots, a_{N}\right]$ that indicate the positive symbol values in a specific component I or Q (real or imaginary part, respectively), where $N$ 


\begin{tabular}{|r|c|l|}
\hline Constellation & DoF & Parameters to optimize \\
\hline 16NUC & 1 & $a_{1}$ \\
\hline 64NUC & 3 & $a_{1}, a_{2}, a_{3}$ \\
\hline $256 \mathrm{NUC}$ & 7 & $a_{1}, a_{2}, \ldots, a_{7}$ \\
\hline $1024 \mathrm{NUC}$ & 15 & $a_{1}, a_{2}, \ldots, a_{14}, a_{15}$ \\
\hline $4096 \mathrm{NUC}$ & 31 & $a_{1}, a_{2}, \ldots, a_{30}, a_{31}$ \\
\hline
\end{tabular}

Table 3.1: Degrees of freedom and constellation symbols to optimize with 1D-NUCs.
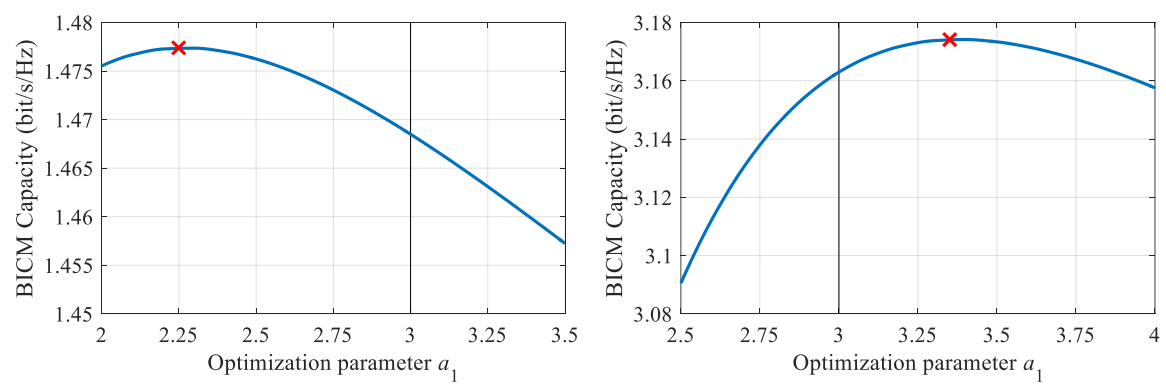

Figure 3.1: BICM capacity values obtained for different parameters $a_{1}$, for 1D-16NUC in AWGN at 3 and $10 \mathrm{~dB}$ of SNR. The value $a_{1}=3$ represents the uniform QAM value.

represents the total DoF. Due to the constraint of power normalization, the smallest value $a_{0}$ is set to 1 . For an SNR target, the BICM capacity is calculated as a function of a. From this parameter, it is necessary to generate the sequence $\left\{-a_{N},-a_{N-1}, \ldots,-a_{1},-1,1, a_{1}, \ldots, a_{N-1}, a_{N}\right\}$ that gives the particular positions of constellations symbols in each component. Note that with QPSK the DoF is $N=\frac{\sqrt{4}}{2}-1=0$, and there is no possible optimization with this constellation. Constellations with 16 cardinality points are the simplest case to evaluate.

\section{The Simplest Use Case: 1D-16NUC}

Assuming a uniform 16QAM with positions $\{-3,-1,+1,+3\}$ on each axis, prior to power normalization, then it is possible to optimize a $1 \mathrm{D}-16 \mathrm{NUC}$ with positions $\left\{-a_{1},-1,+1,+a_{1}\right\}$, using a single parameter $a_{1}$. For example, Figure 3.1 shows the BICM capacity of a $16 \mathrm{NUC}$ at SNRs of 3 and $10 \mathrm{~dB}$, for AWGN channel. A parameter $a_{1}$ equal to 3 corresponds to the uniform case, while the maximum capacity is obtained for values of $a_{1}$ of 2.25 and 3.35 respectively. After obtaining $a_{1}$, the set of constellations can be pertinently normalized.

It is very important to highlight that the optimization process is burdensome, especially when increasing the number of parameters. Rather than con- 

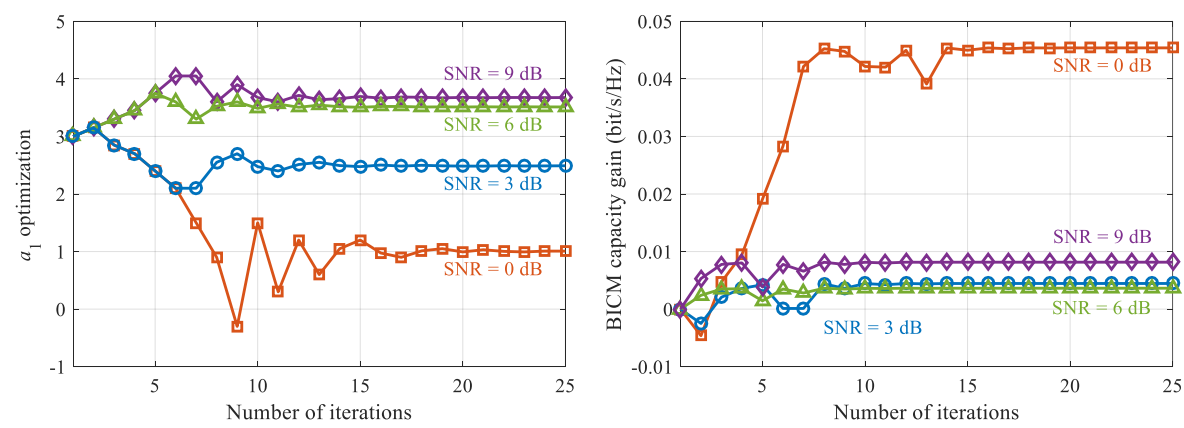

Figure 3.2: Optimization of $a_{1}$ with the Nelder-Mead algorithm, for 1D-16NUC in AWGN at different SNRs (left). BICM capacity improvements are also shown (right).

sidering all possible values, it is better to use an algorithm that automatically finds the optimum parameters, maximizing the BICM capacity for each SNR by numerical optimization. In this dissertation, we use the Nelder-Mead algorithm, which is detailed in [89] and proposed in [38] for designing NUCs. As reference [101] mentions, the method minimizes a function of $n$ variables, based on the comparison of function values at the $(n+1)$ vertices of a general simplex, followed by the replacement of the vertex with the highest value by another point. The simplex adapts itself to the local landscape, searching for the final minimum. The mathematical approach of this method is detailed in Appendix B, where it is shown to be efficient and computationally compact.

Fig. 3.2 provides four examples of optimization using this algorithm. From QAM values $\left(a_{1}=3\right)$ and after 25 iterations, the method reaches the optimum solution. The BICM capacity gain achieved along the whole search is also shown for comparison. It can be observed that $16 \mathrm{NUCs}$ optimized for very low SNRs provide higher gains than the rest. Selecting the values of $a_{1}$ yielding the maximum capacity for a large range of SNRs can provide the basis for the construction of a 16NUC adaptive to a particular channel model [33]. As a consequence of the dependence of the NUC symbols on the SNR, each constellation cannot provide the maximum gain for all operation points, and therefore CRs. Hence, a specific NUC is defined for each CR from the evaluated SNR. In particular, when considering strong error correcting codes such as an LDPC, the target SNR of the NUC is selected for each CR according to the SNR of the waterfall region.

One can ideally think of designing a combined NUC that provides the best possible capacity for fixed and mobile receptions at the same time. The left part of Fig. 3.3 depicts the optimum parameter for channel models typical from 

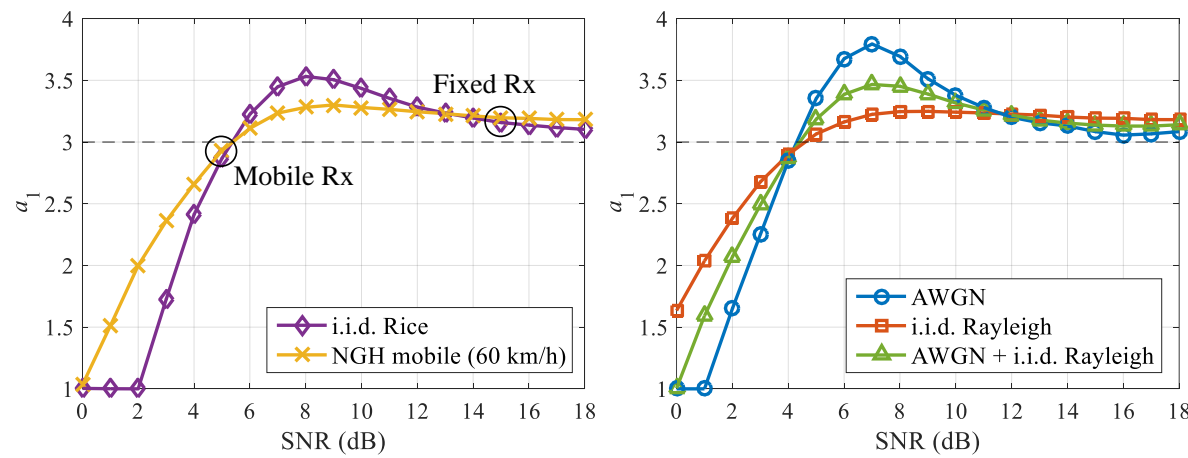

Figure 3.3: Optimization parameter $a_{1}$, as a function of the SNR, for i.i.d. Rice and NGH mobile channels (left). Results are also provided for AWGN, i.i.d. Rayleigh, and their combination (left).

broadcasting communications, i.e. NGH mobile with speed $60 \mathrm{~km} / \mathrm{h}$ and i.i.d. Rice, which are defined in Appendix A. Assuming values of 5 and $15 \mathrm{~dB}$ for mobile and fixed channels respectively, we seek the optimum parameter that provides the best combination of both capacities, i.e. $a_{1}=3.12$. However, since both modes require totally different SNR ranges, this combination is not optimum at all. It is better to simply focus on a particular SNR.

The right part of the figure depicts the results for AWGN and i.i.d. Rayleigh channels. In this case, two different approaches are considered. The first option is to optimize constellations for each channel separately. The resulting constellations provide the maximum capacity for the case under study, but they do not give an optimum result when considering another channel model. As a compromise, both optimizations can be combined in a single calculation, for each SNR, where the average capacity of both channels is maximized. As can be observed, the parameter obtained when considering both channels is approximately the average of the parameters obtained in separated optimizations. In this section, we always calculate the combined values for these two channel models, which were already considered in the ATSC 3.0 standardization process [17].

Note that in any case, the parameter $a_{1}$ converges to 1 at low SNRs. Constellations collapse to QSPK, and identical symbols are grouped in clusters. The idea is to transmit a QPSK-like constellation but with more bits per symbol, since the MSB provide similar robustness but the LSB are used to give additional information. On the contrary, the use of a traditional QPSK allows to double the coding rate, so in practice both constellations provide similar performance [34]. Another feature to take into account is power consumption. 

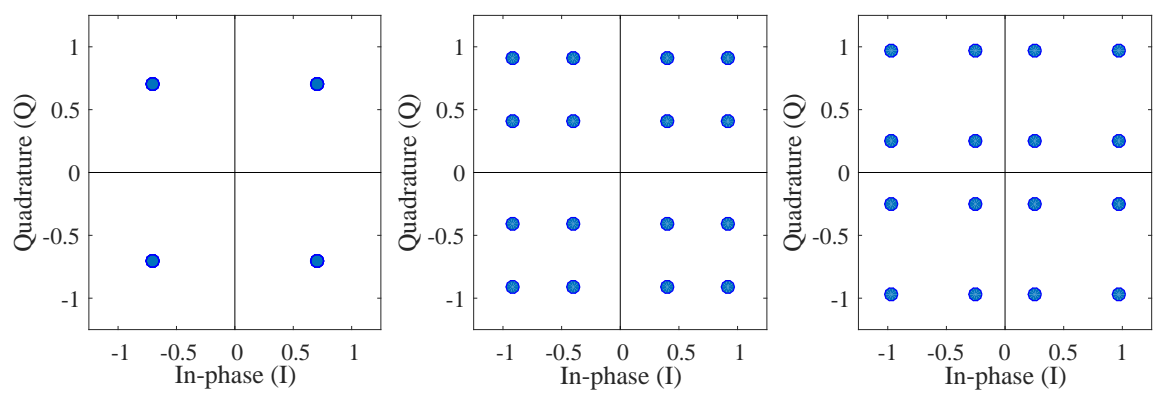

Figure 3.4: Different one-dimensional 16NUCs, optimized for SNRs of 0, 3 and $6 \mathrm{~dB}$, for AWGN and i.i.d. Rayleigh channels.

Although condensed, high-order constellations increase the power necessary to transmit all possible symbols. From this point of view, it is better to transmit low constellation orders that allow to use similar spectral efficiencies by using higher CRs. At high SNRs, the positions converge toward a uniform shape. Without a robust $\mathrm{CR}$, the best option is to pack the constellation symbols as uniformly spaced as possible. The overall tendency can be found in Fig. 3.4, where we show different 1D-16NUCs designed for SNR values of 0,3 and $6 \mathrm{~dB}$ and both AWGN and i.i.d Rayleigh channels, after power normalization.

\section{Extrapolation to High-Order Constellations}

Larger number of DoF gives much greater improvement than 16NUC, compared with their uniform versions. Consequently, larger NUCs get progressively closer to the theoretical Shannon limit, but the algorithm needs for additional iterations to find the optimum solution.

When 64 NUCs are considered, 3 different parameters $\left(a_{1}, a_{2}, a_{3}\right)$ need to be optimized, as Table 3.1 shows. As done with 16NUCs, we consider the QAM positions as initial variables, since the complexity is not really high and therefore no local minimums are reached by the algorithm. Fig. 3.5 depicts the variation in the optimum symbol positions when the BICM capacity of the particular NUC is optimized at different SNRs. The dashed lines with values 3, 5 and 7 indicate the conventional uniform QAM position. Overall, variations with SNR are similar to $16 \mathrm{NUC}$, but obtaining a higher level of condensation. At $5 \mathrm{~dB}$, the constellation fully converges to a $16 \mathrm{NUC}$. At $0 \mathrm{~dB}$, 64NUCs collapse into QPSK constellations if the SNR is low enough, so the there are two levels of condensation. On the other hand, at high SNR values the positions converge towards the uniform QAM values. 


\subsection{Non-Uniform Constellations Optimization}

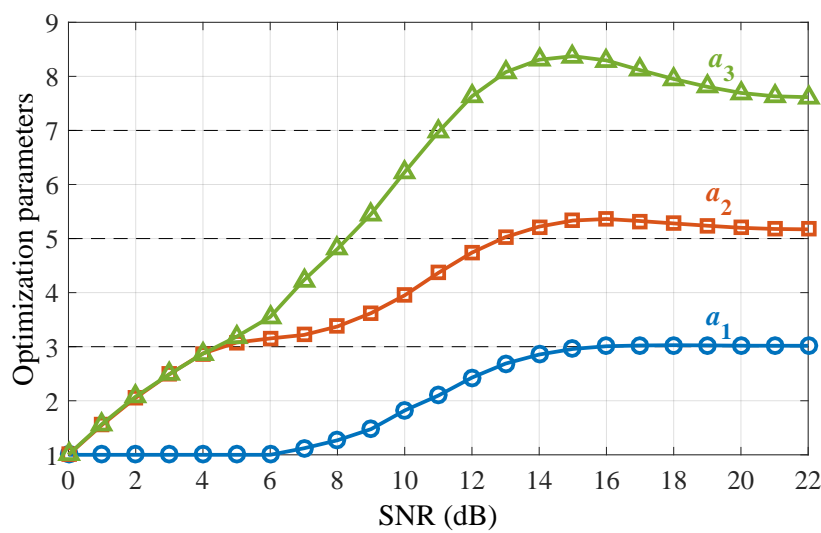

Figure 3.5: Optimization parameters for 64NUC, as a function of the SNR, for the combination of AWGN and i.i.d. Rayleigh channels.

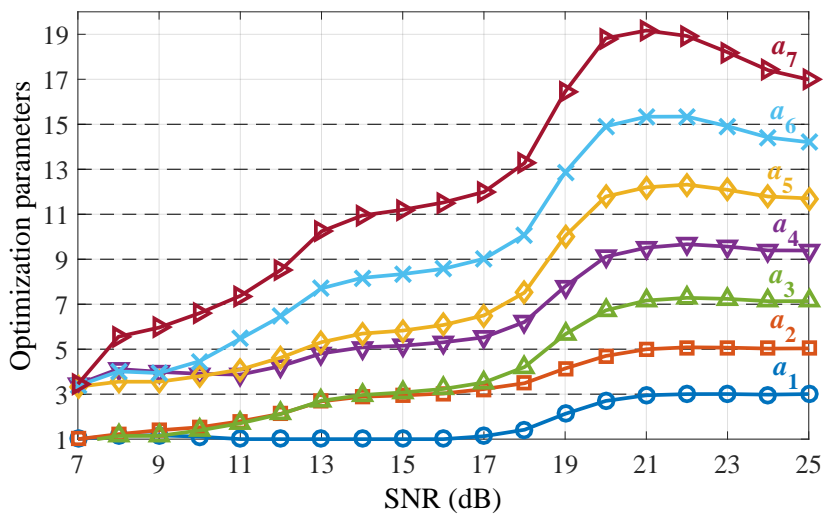

Figure 3.6: Optimization parameters for 256NUC, as a function of the SNR, AWGN channel model.

256NUCs follow a similar tendency, as Fig. 3.6 shows. Several regions can be differentiated. As occurred in previous results, 256NUC constellations tend to approach the uniform QAM values at high SNRs, and at the same time, while the SNR decreases the symbol positions become condensed and symbols with lower values begin to merge. The constellation does in effect reduce its number of symbols, grouping them in clusters, going from 256QAM positions down to non-uniform values of $16 \mathrm{NUC}$, at about $7 \mathrm{~dB}$ SNR. 


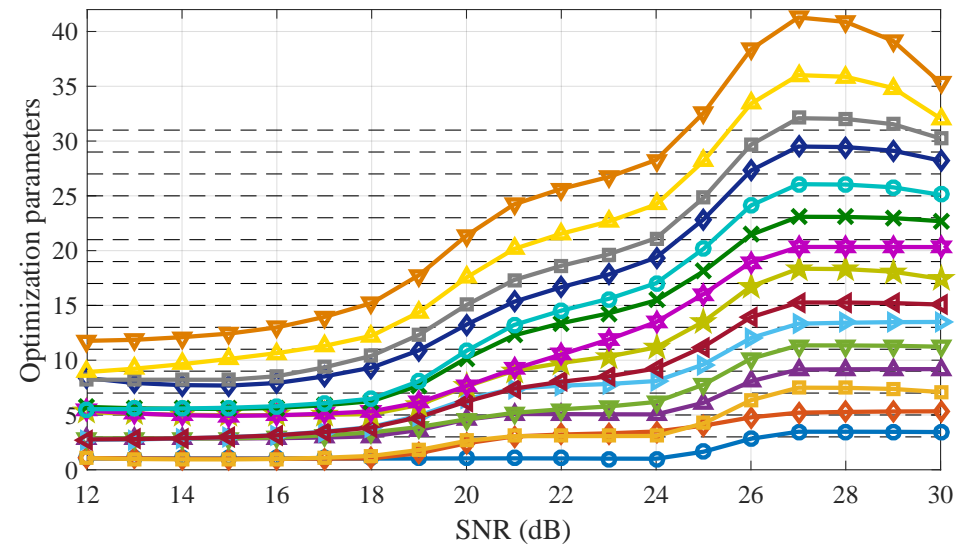

Figure 3.7: Optimization parameters for 1024NUC, as a function of the SNR, AWGN channel model.
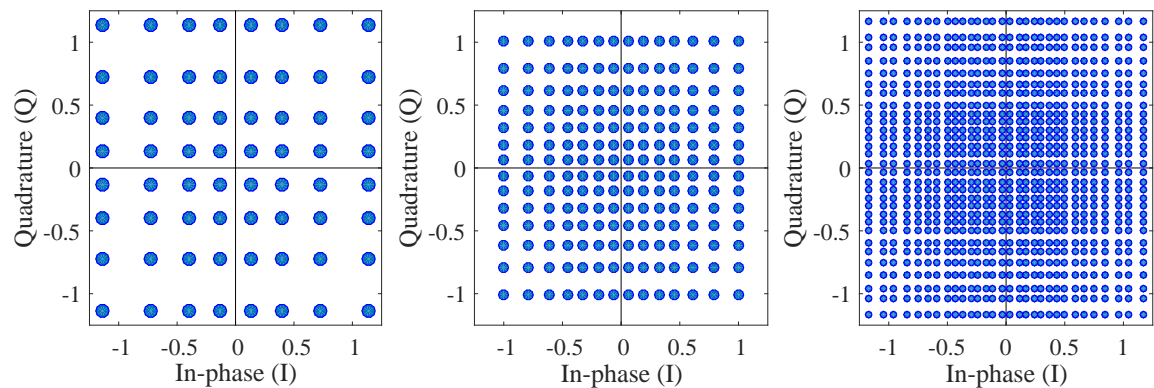

Figure 3.8: Examples of 64NUC, 256NUC and 1kNUC optimized for SNRs of 15, 20 and 25 $\mathrm{dB}$ respectively, for the combination of AWGN and i.i.d. Rayleigh channels.

With 1024NUCs, the number of parameters becomes higher, with $N=15$. Fig. 3.7 shows the optimum parameters for these constellations, optimized for SNRs from 12 to $30 \mathrm{~dB}$, and AWGN channel. Three different regions can be differentiated here. First, at low SNRs (range from 12 to $20 \mathrm{~dB}$ ) half or more of the symbols collapse and merge. Concretely, $a_{1}, a_{2}$ and $a_{3}$ have a similar value $1 ; a_{4}, a_{5}, a_{6}$ and $a_{7}$ merge to $3 ; a_{8}, a_{9}, a_{10}$ and $a_{11}$ converge to 5 , and the rest keep different values, although much lower than QAM. The second region between 21 and $25 \mathrm{~dB}$ expands some of the symbols, with a lower compression but still under the uniform conventional QAM. Finally, above $25 \mathrm{~dB}$, all the constellation symbols are different, so there is no condensation in this case. 


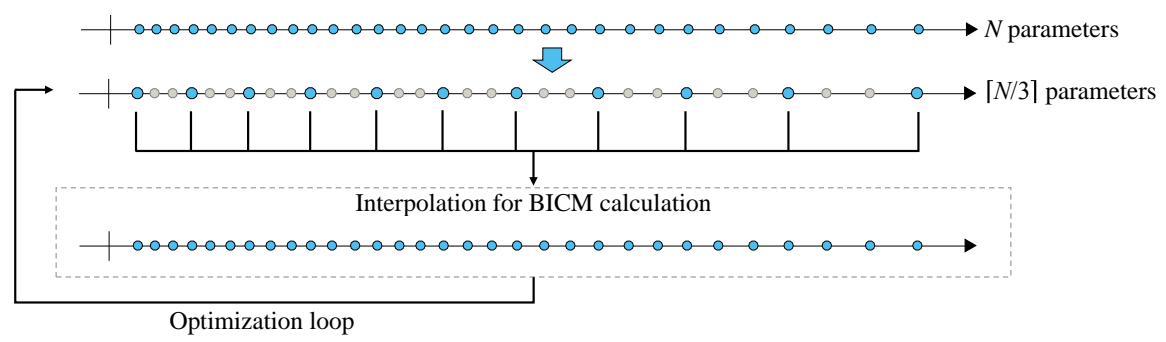

Figure 3.9: Optimization $N / D_{R}$ method, with $D_{R}=3$. Reduction from 31 to 11 parameters to be optimized.

Fig. 3.8 shows three different examples for the different orders considered, i.e. $64 \mathrm{NUC}, 256 \mathrm{NUC}$ and $1 \mathrm{kNUC}$ optimized for SNRs of 15, 20 and $25 \mathrm{~dB}$ respectively.

\section{The Particular Case of 4096NUC: A Different Approach}

As mentioned in previous section, the use of very high-order constellations requires a large number of variables to optimize. As a consequence, the optimization burden is incremented, and also to find a global minimum in the optimization algorithm becomes harder. Up to $1024 \mathrm{NUC}$, the number of optimization parameters is lower enough. However, with 4096NUC things get complicated. To optimize 31 parameters guides sometimes the algorithm to local minimums that do not reach the optimum solution. In order to avoid it, and to reduce the number of iterations, a new low-complexity method can be used. We call this method the Optimization $N / D_{R}$, where $N$ is the initial number of degrees of freedom and $D_{R}$ is the decimate ratio. Fig. 3.9 helps to understand the proposed method, when $D_{R}=3$. Initial $N$ parameters are reduced to $\left\lceil N / D_{R}\right\rceil$, where $\lceil\cdot\rceil$ represents the first integer rounded up. In other words, instead of taking all parameters as inputs to the optimization algorithm, it is only necessary to take 1 out of $D_{R}$. Afterwards, the optimization loop starts, and the rest of parameters are just derived with a shape preserving piecewise cubic interpolation [102] inside the algorithm, when constellations are configured. The BICM capacity is then calculated and used as output, and the process is repeated iteratively as usual.

A more elaborated optimization method reduces the possibility of obtaining a local minimum. It also halves, on average, the number of iterations to reach the optimum solution. In this dissertation, we use this method to reach a suboptimum solution. Afterwards, the positions obtained for all 31 parameters 

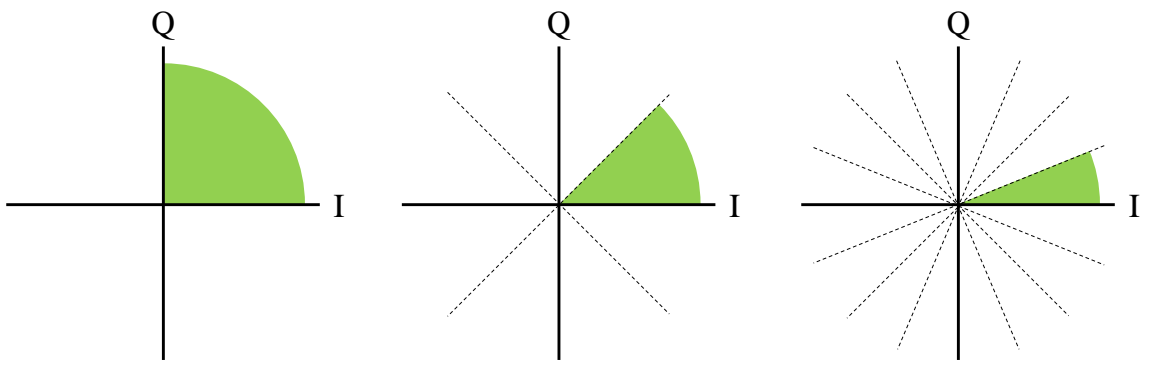

Figure 3.10: 2D-NUC optimization when considering different symmetries: 4 sectors (left), 8 sectors (center) and 16 sectors (right).

are used in a new re-optimization, which obtains the optimum constellation and slightly increases the BICM capacity.

\subsubsection{Two-Dimensional Non-Uniform Constellations}

The use of one-dimensional NUCs has different advantages when optimizing. First, imposing a squared shape where both I and Q components can be separated drastically reduces the number of parameters to optimize. The simple symmetry imposed about the center further simplifies the process. Both simplifications facilitated the optimization of constellations up to 4096 symbols. However, these advantages cause some limitations at lower SNRs, where larger gains could be obtained.

With 2D-NUCs, constrains imposed change and therefore the process has to be redefined. In this thesis, we follow the approach given by [43], where 2D-NUCs are designed by relaxing the square shape constraint of 1 D-NUCs and QAM constellations. Thanks to that, it is possible to obtain a better performance but with a higher receiver complexity, since 2D-NUCs cannot be separated into two independent I/Q components. It is assumed that constellations retain left-right and up-down symmetry. The design process can be reduced by defining just the first quarter of the complex symbols, with the rest being derived by just using both $\mathrm{I} / \mathrm{Q}$ axes as mirrors. This entails a large reduction in both the DoF, as shown in Fig. 3.10 (left), and the number of iterations.

Additional symmetries can be added at the expense of some SNR loss, but still obtaining better capacities that those obtained for 1D-NUCs. It is possible to add greater levels to the optimization algorithm, so that the computation complexity can be highly reduced, being able to optimize high-orders of constellations [43]. In principle, the highest BICM capacity is obtained when opti- 


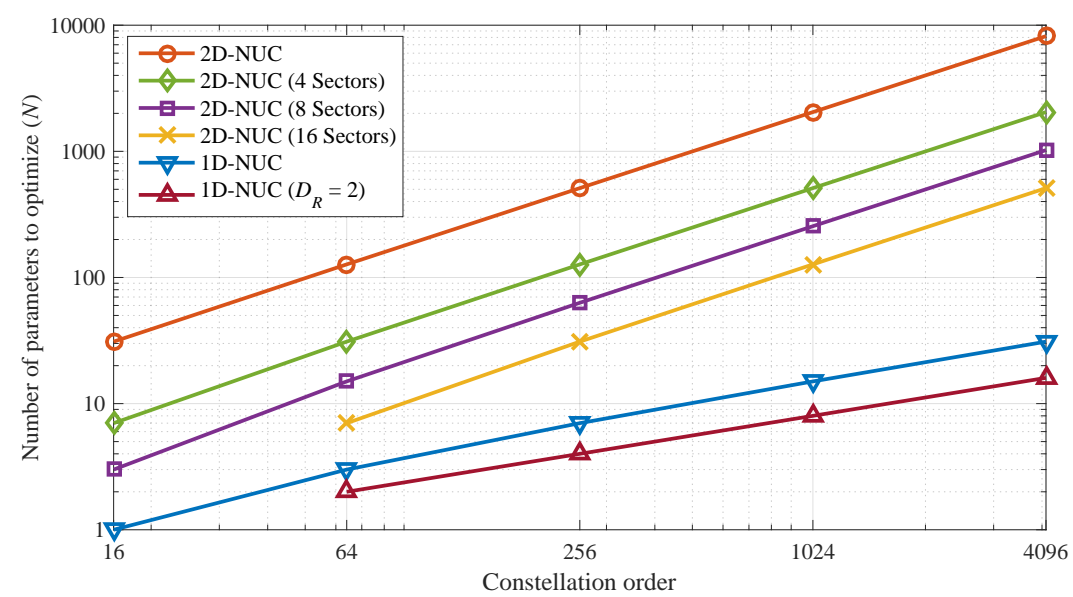

Figure 3.11: Number of parameters to optimize, for different orders of constellation.

mizing an unconstrained 2D-NUC, but computing it extremely complex, while the capacity improvement is negligible. In this dissertation, some constellations are first optimized using 4, 8 and 16 sectors, depending on the order and SNR, and afterwards re-optimized for a lower number of sectors (in order to avoid local minimums). Fig. 3.10 shows the constellation diagrams and the different symmetries considered. Green areas represent the symbols that are optimized.

The total degrees of freedom increase, compared to 1D-NUCs. Optimizing in one dimension maximizes the BICM capacity by changing the positions of $N=\left(\frac{\sqrt{M}}{2}-1\right)$ symbols. This number can be further simplified if the proposed optimization $N / D_{R}$ is applied, for different values of $D_{R}$. With $2 \mathrm{D}-\mathrm{NUCs}$, the symbols need to be specified in two dimensions, so two free real variables are considered. Real and imaginary parts constitute the new variables to optimize, i.e. $2 M-1$ (due to the constraint of power normalization). The number of variables when using the different symmetries presented above is reduced to $\left(\frac{2 M}{S}-1\right)$, where $S$ represents the number of sectors. The use of 4,8 or 16 sectors reduces the number of variables to $\left(\frac{M}{2}-1\right),\left(\frac{M}{4}-1\right)$ and $\left(\frac{M}{8}-1\right)$ respectively. A good comparison between the different types of optimization already proposed can be found in Fig. 3.11. In this dissertation, we first optimize constellations so the total number of parameters is 31 or less. For those cases in which the optimization may reach a suboptimum solution, a new re-optimization with more variables to look for the optimum solution is done. 

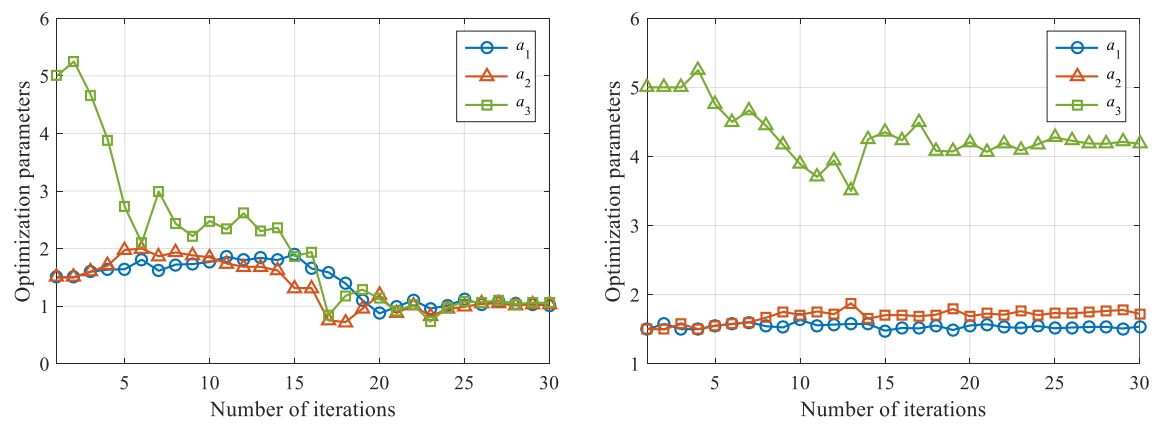

Figure 3.12: 8 sectors optimization with the Nelder-Mead algorithm, for 2D-16NUC in AWGN with two different SNR values: $0 \mathrm{~dB}$ (left) and $5 \mathrm{~dB}$ (right).

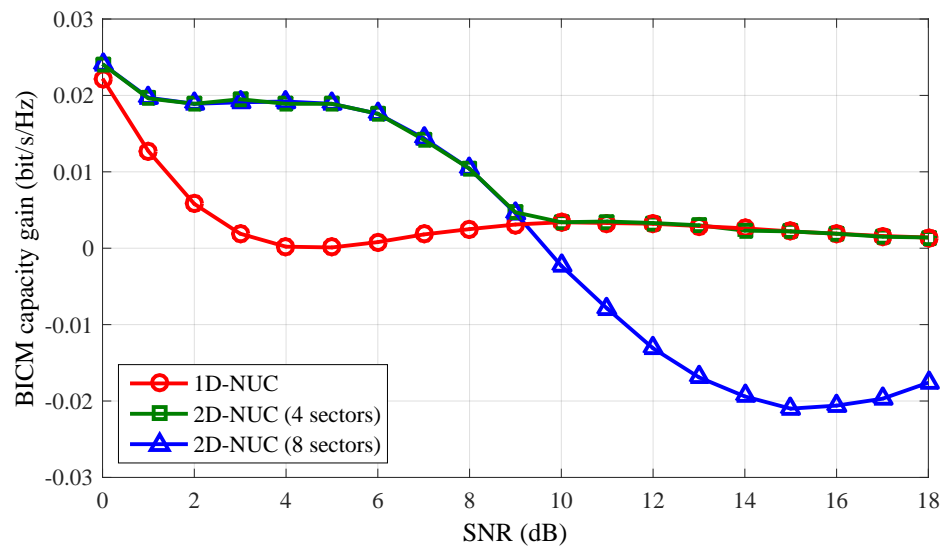

Figure 3.13: BICM capacity gain achieved with 1D-NUC and 2D-NUC (considering 4 and 8 sectors).

\section{D-16NUC Optimization}

At low SNRs, constellations collapse into a QPSK, as occurred with 1D-NUC. The BICM capacity improvement is therefore the same for both cases. At medium SNR values, constellations change towards a Gaussian shape that satisfies the 8 sectors symmetry, so the optimization burden can be further reduced. In this region, the highest BICM capacity improvements are achieved.

Fig. 3.12 provides two examples of optimization when considering this symmetry, for SNRs of 0 and $5 \mathrm{~dB}$. From the same random initial values and after 30 iterations, the method reaches the optimum solution. In the examples 

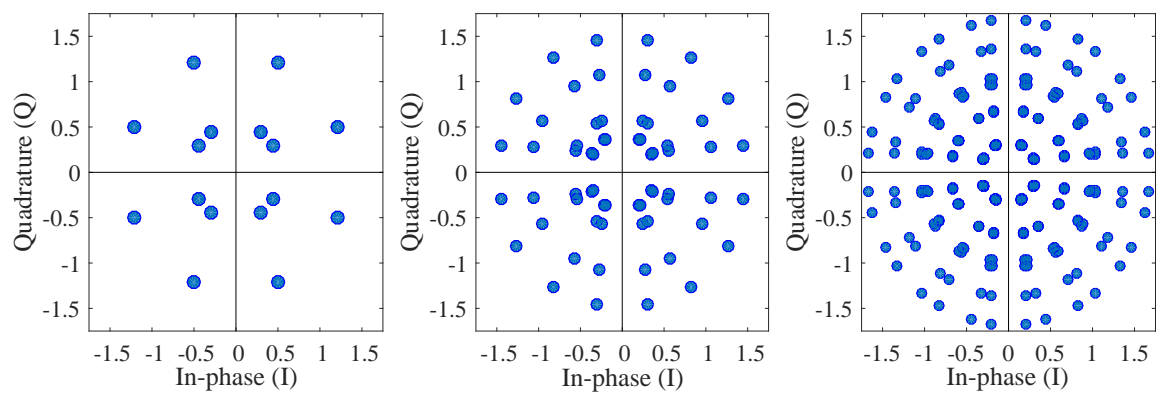

Figure 3.14: Two-dimensional 16NUC, $64 \mathrm{NUC}$ and 256NUC, optimized for SNRs of 5, 10 and $15 \mathrm{~dB}$, for the combination of AWGN and i.i.d. Rayleigh channels.

provided, three different parameters are required. Note that all parameters tend to 1 for a SNR of $0 \mathrm{~dB}$, collapsing towards a QPSK and thus having the same shape than 1D-NUC. The optimized 2D-NUC for the SNR value of $5 \mathrm{~dB}$ is shown in 3.14 (left). As the SNR increases, 16NUCs undergo a clear modification in shape between 9 and $10 \mathrm{~dB}$, changing from a Gaussian shape to a square 1D constellation. This concrete evolution can be seen in Fig. 3.13.

The optimum solution at high SNR ranges is therefore a $16 \mathrm{NUC}$ with squared shape, similar to $1 \mathrm{D}$, where almost in all cases constellation symbols can be separated into two different I and Q components. The behavior comes from the relationship between high SNRs and high CRs. At very high SNRs, coding tends to unity where no redundancy bits are employed. Since there is no benefit from that coding, a uniform separation between symbols ensures the minimum distances between them, minimizing the errors as much as possible.

\section{D-64NUC Optimization}

Two-dimensional optimizations of $64 \mathrm{NUC}$ constellations need a total of $N=31$ parameters, if 4 -sectors symmetry is considered. In case of further simplification, where 8 sectors are taken into account, the number of parameters is reduced to 16 . In this thesis, we first design 2D-64NUCs in a more-constrained 8-sectors optimization. Afterwards, a 4-sectors re-optimization is performed, in which some symbols change their position in order to achieve higher capacities.

Fig. 3.14 (center) shows a 2D-64NUC optimized for a SNR of $10 \mathrm{~dB}$. As reference [43] affirms, these constellations have a circular shape composed of rings and radials, but in fact radials are not radials, since they do not pass through the origin. They do not have rings either, due to the re-optimization performed in 4 sectors, which gives a higher freedom to constellation symbols to find other positions. As occurred with 1D, at low SNRs constellations collapse 


\section{CHAPTER 3. OPTIMIZATION AND PERFORMANCE EVALUATION OF NON-UNIFORM CONSTELLATIONS}

into low-order NUCs. In this case, symbols are grouped so that the constellation has a 16NUC-like shape. If the SNR is low enough, these constellations tend to QPSK. Results derived from both methods are identical for all SNRs below 12 $\mathrm{dB}$. The re-optimization in 4 sectors starts to take effect from $13 \mathrm{~dB}$. Note that, unlike 16NUCs, 64 NUCs do not tend to the one-dimensional version of these constellations, even for very high SNRs. Despite the circular shape, all symbols keep a good almost-uniform spacing, which is also a good way to achieve the highest capacity at these SNR values. What happens with $16 \mathrm{NUCs}$ is that they have less margin, so the best option is just to merge to 1D-NUC.

\section{D-256NUC Optimization}

The optimization process becomes really hard from $256 \mathrm{NUC}$ on. In fact, only up to 2D-256NUCs were considered in the ATSC 3.0 standardization process. Observing Fig. 3.11, the number of parameters to optimize in a $256 \mathrm{NUC}$ when only 4 sectors are considered goes up to 127 , which is not feasible from an optimization point of view. The very large number of input variables yields into finding local minimums, after a very long number of iterations (orders of 1000). Moreover, the use of a two-dimensional demapper in which 256 distances need to be computed, increases the complexity burden even more. For this reason, some assumptions must be taken, in order to reduce the optimization complexity and obtain realistic results. It is therefore clear that these constellations need higher degrees of symmetry, and even sometimes prior-condensation assumptions.

In this dissertation, we first assume 16 sectors, in order to reduce the number of variables to 31. For low SNRs, and observing the tendencies in low-order constellations, some symbols were grouped in clusters, reducing the number of variables down to 15-20 (depending on the particular SNR). Imposing 16sectors symmetry results in optimization complexity comparable to 2D-64NUC, or 1D-4096NUC, although with different demapping complexities. Once those preliminary constellations are obtained, two additional steps are performed. First, a new re-optimization with 8 sectors and therefore 63 parameters is performed. This re-optimization sometimes does not give an additional capacity, due to the very high number of parameters. The second step is to look for the optimum positions of the inner symbols (from the two first rings of the constellation), since they usually change their position and break the symmetries imposed, especially for high SNRs. Fig. 3.14 (right) shows an example of a 2D-256NUC optimized for a SNR of $15 \mathrm{~dB}$. As occurred with $64 \mathrm{NUCs}$, they seem to have a circular shape, with several rings and radials, but in fact they do not keep those constrains. As usual, the 2D-NUC optimized for low SNR values tends to lower orders of constellations. As denoted in [43], the 256NUC 


\subsection{Non-Uniform Constellations Optimization}

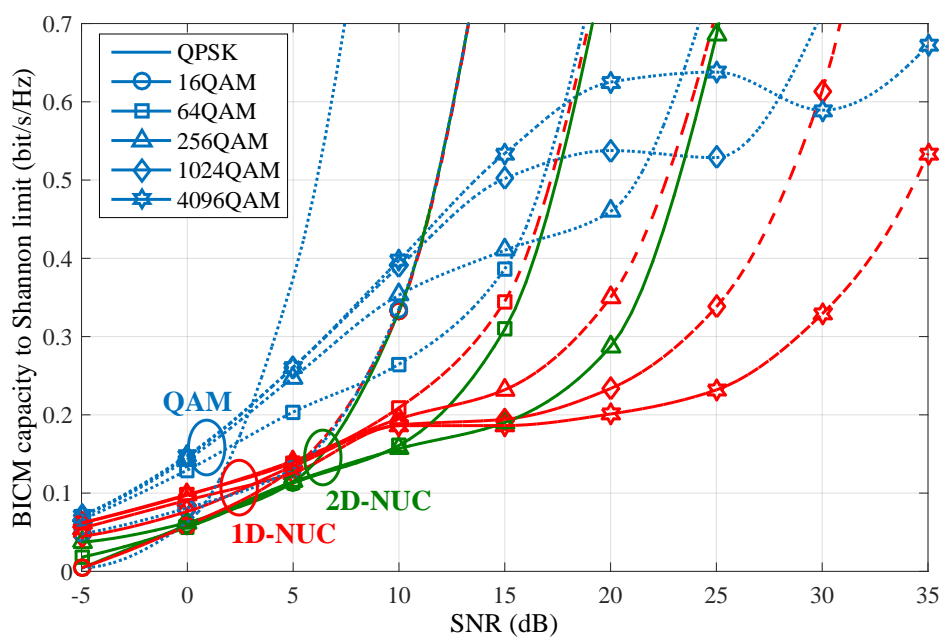

Figure 3.15: Shortfall of the BICM capacity from the unconstrained Shannon capacity, for the i.i.d. Rayleigh channel and a range of Gray-mapped QAM and 1D/2D-NUC constellations.

optimized for a SNR of $10 \mathrm{~dB}$ is in practice very similar to a $64 \mathrm{NUC}$, where the three first rings of inner symbols repeat several positions. Paying more attention, there are 18 different and recognizable symbols per quadrant, so the constellation has in practice 72 different symbol positions. For a medium SNR of $15 \mathrm{~dB}$, the number of recognizable symbols goes up to 120 , and even at high SNRs some symbols still share some positions.

\subsubsection{BICM Capacity Improvements}

Up to now, this chapter has explained the optimization process for both 1D- and 2D-NUCs, as well as shown some examples of the final constellations obtained. In this section, the BICM gain in bit/s/Hz provided by these constellations is shown. 1D constellations from 16 to 4096 cardinality symbols, and 2D from 16 to 256 are considered. Fig. 3.15 shows the BICM capacity to the Shannon limit given by uniform QAM vs. optimized NUCs. In this case, all 1D- and 2D-NUCs are properly optimized for a range of SNR values from -5 to $35 \mathrm{~dB}$. Results are shown for the i.i.d. Rayleigh channel. The use of this channel model is justified since terrestrial channels are typically Rice or Rayleigh distributed, as a consequence of terrestrial multi-path propagation. Each NUC performs better at its design SNR than its uniform variant, overall at high orders. Moreover, at any SNR, there is always a particular NUC that maximizes the greatest BICM capacity. The blue pointed lines denote the uniform QAM constellations, which 


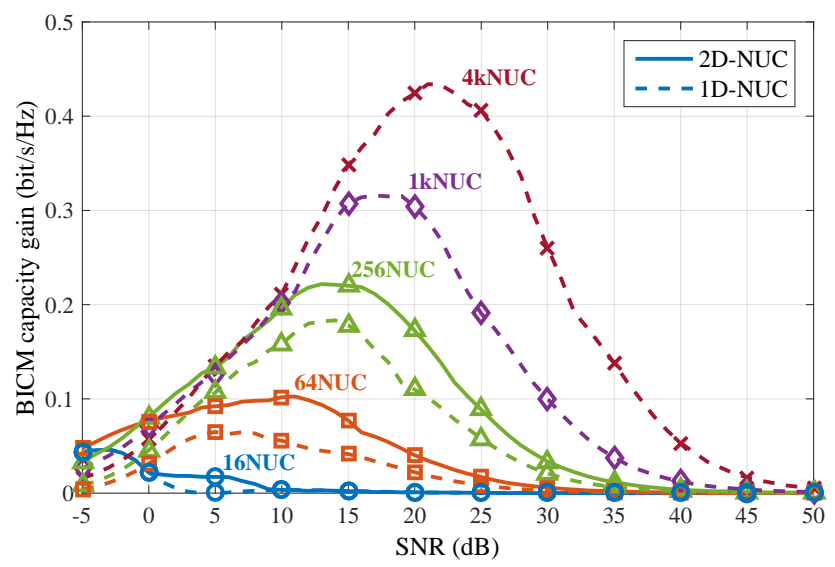

Figure 3.16: Gain of 1D/2D-NUC over QAM, for the i.i.d. Rayleigh channel model, and a range of SNR values between -5 and $50 \mathrm{~dB}$.

shows how the successive uniform sizes take turns to be the best option as the SNR increases. The red dashed lines and green solid lines show the results for $1 \mathrm{D}$ and 2D-NUCs respectively.

As Fig. 3.15 shows, 2D-NUCs are always a better option than 1D-NUCs from the capacity point of view. In general terms, two general conclusions can be formulated. First, the BICM capacity gain increases with the constellation order, regardless of the type of NUC used. Second, the relative gain between 2D- and 1D-NUCs is always higher for the medium range of SNRs in which each order of constellation works. Logically, the SNR range also increases with the constellation order. This behavior can be better observed in Fig. 3.16, which shows the BICM capacity gain of 1D-NUCs (pointed lines) and 2D-NUCs (solid lines) for a complete range of SNRs, from -5 to $50 \mathrm{~dB}$.

From the results for $16 \mathrm{NUC}$ in Fig. 3.16, it is possible to affirm that both $1 \mathrm{D}$ and 2D optimization give the same gain at low SNRs. This is due to the condensation that both suffer, reaching the same result independently on the parameters optimized, i.e. a QPSK constellation. In the range of 2 to $8 \mathrm{~dB}, 2 \mathrm{D}$ significantly outperforms $1 \mathrm{D}$. Note that in the range where the capacity gain is really useful, there is no penalty for the use of a 8-sector optimization. At high SNRs, both 16NUCs converge to the uniform QAM distribution, and the gain becomes zero. Similar results are obtained for 64 NUCs, although the SNR range changes. In this case, there is wide range where $2 \mathrm{D}$ offer significant gains compared to $1 \mathrm{D}$, from -5 to $25 \mathrm{~dB}$. At very low SNRs, they collapse into QPSK and, therefore, the BICM capacity gain provided is the same than 16NUC. 2D- 
64 NUCs offer a high benefit up to $15 \mathrm{~dB}$. From that value, $2 \mathrm{D}$ constellations gain merges towards the $1 \mathrm{D}$ results. However, even at the highest SNRs of 30 or $40 \mathrm{~dB}, 2 \mathrm{D}$ does give a tiny advantage over $1 \mathrm{D}$. Observing the curves obtained for $256 \mathrm{NUC}$, the $2 \mathrm{D}$ gain compared to $1 \mathrm{D}$ is maintained from -5 to $20 \mathrm{~dB}$, being around $0.03 \mathrm{bit} / \mathrm{s} / \mathrm{Hz}$. It is interesting to see that at very low SNRs, the gains obtained are lower than those reached by 16 and 64NUCs. At high SNRs, as occurred with $64 \mathrm{NUCs}, 2 \mathrm{D}$ constellations gains tend towards the 1D results. Both higher SNR ranges and BICM capacity gains are obtained for $1 \mathrm{kNUC}$ and $4 \mathrm{kNUC}$. Observing Fig. 3.16, gains up to $0.43 \mathrm{bit} / \mathrm{s} / \mathrm{Hz}$ are obtained for medium SNRs with $4 \mathrm{kNUCs}$, which can give performance gains on the order of 1-1.5 dB, if the SNR target and CR are accordingly selected. Performance results for the optimized constellations are shown in Section 3.4.

\subsection{Non-Uniform Rotated Constellations}

Results in previous sections have shown that the gain of NUCs becomes almost non-existent at high SNRs, especially when optimizing for fading channels. In this particular case, rotated constellations can be used to improve the overall system performance [25]. With RCs, a certain rotation angle is applied to the constellation, so that the binary information is transmitted simultaneously in different I and $\mathrm{Q}$ components. In order to ensure that each component undergoes independent fading, a Component Interleaver (CIL) is applied after the rotation to separate both components in time and frequency domains. Thus, it is possible to retrieve all the information at the receiver side even when one of the components has been erased by the channel. With NUCs, it is required to optimize different rotation angles, since they are optimized so that the diversity experimented by both I and Q components is maximized.

When applying an additional rotation to a NUC, there are two different possibilities [103]. The simplest way is to optimize first the constellation symbols and apply afterwards a rotation, at the expense of a penalty loss (constellations are optimized without including the rotation). We call this method Optimization Before Rotation (OBR). As an improved solution, it is possible to include the rotation angle as a new variable in the optimization process. This second method is called Optimization with Additional Rotation (OAR). Both methods are explained in the following sections.

In a single RF transmission, the rotation gain is only significant for some particular combinations, i.e. low order constellations and high code rates, as reference [104] shows. However, RCs may become especially effective when using multi-RF techniques, such as Channel Bonding (CB) [62], providing very high performance gains. CB has been adopted in ATSC 3.0 and consists of 

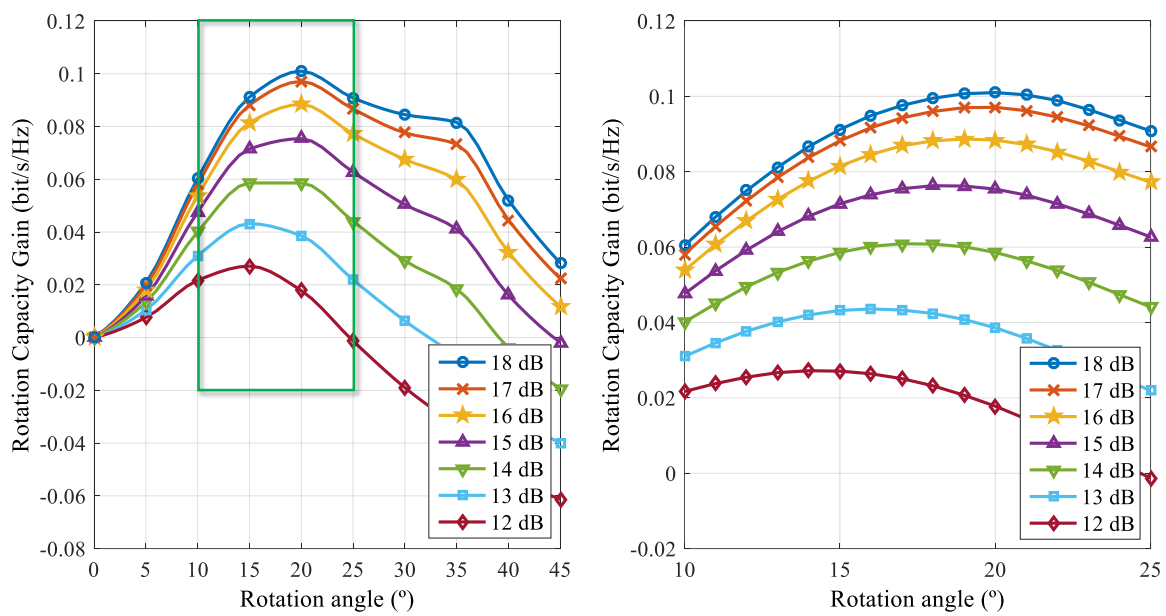

Figure 3.17: BICM Capacity gain of 2D-16NURCs, depending on the rotation angle and SNR.

splitting service data across two RF channels, so that peak data rate can be doubled. In such case, it is desirable that each component is transmitted in a different RF channel using a proper CIL, which must be redesigned. Results can be also extended to any multi-RF technology, such as Time-Frequency Slicing (TFS) [60]. TFS was proposed in DVB-T2 and adopted in DVB-NGH (Next-Generation Handheld) [14]. With TFS the data is transmitted in a slotby-slot manner by frequency hopping across an RF-Mux of two or more RF channels (in practice, up to 6). The use of NURCs in connection with multi-RF techniques may become a good solution to increase robustness.

\subsubsection{Optimization Before Rotation}

Generally speaking, with rotated constellations the demapper has to consider all symbols in both dimensions, regardless of the constellations shape. Comparing 1D- and 2D-NURCs, it is better to use 2D-NURCs, as they always provide the best capacity for a given SNR, as shown in Fig. 3.16. In addition, the rotation of 2D-NUCs does not increase the demapping complexity, since a 2Ddemapper is also needed. A typical $2 \mathrm{D}$ rotation is performed by multiplying two real (I and Q) components by an orthogonal rotation matrix of size $2 \times 2$, as shown in Eq. 3.1. 


$$
\left[\begin{array}{l}
\operatorname{Re}(y) \\
\operatorname{Im}(y)
\end{array}\right]=\left[\begin{array}{ll}
+a & -b \\
+b & +a
\end{array}\right]\left[\begin{array}{l}
\operatorname{Re}(x) \\
\operatorname{Im}(x)
\end{array}\right]
$$

where $x$ is the original symbol, $y$ is the resulting rotated symbol, $a=\cos \left(\frac{2 \pi \theta}{360}\right)$ and $b=\sin \left(\frac{2 \pi \theta}{360}\right)$, being $\theta$ the rotation angle in degrees. Selected angles are those that provide the maximum BICM capacity using the i.i.d. Rayleigh channel model.

To understand the effect of the rotation angle on constellations, Fig. 3.17 (left) depicts the BICM capacity obtained when rotating from $0^{\circ}$ to $45^{\circ}$ the 2D-16NUCs designed in Section 3.1.1 for SNRs from 12 to $18 \mathrm{~dB}$. It also shows a zoom to the particular SNR range in which the rotation may become highly beneficial (right), i.e. from $10^{\circ}$ to $25^{\circ}$. Observing the figure in detail, higher SNR values require larger rotation angles. For example, an SNR of $13 \mathrm{~dB}$ requires an angle of $16^{\circ}$ while an SNR of $16 \mathrm{~dB}$ maximizes the capacity with $19^{\circ}$.

These results are the basis for the rest of the section, but it seems obvious that as done for NUC optimization, it is better to use an algorithm that automatically finds the optimum angle. As an example of application, Fig. 3.18 shows the rotation angle optimization process for $16 \mathrm{NURC}$, from an initial angle of $15^{\circ}$ (utilized for QAM constellations in DVB-T2 [12]) and using the Nelder-Mead algorithm [89]. A complete SNR range from 8 to $18 \mathrm{~dB}$ was selected. As occurred before, the higher the SNR target, the larger the rotation angle, with an upper bound of $20^{\circ}$. By contrast, when the SNR is significantly lower, the rotation angle becomes $0^{\circ}$, which implies better to use a non-rotated NUC.

It should be noted that RCs can be specified in two (2D) or four (4D) dimensions, depending on the number of real components in which the rotation is applied. A typical $4 \mathrm{D}$ rotation is performed by multiplying 4 real (two I and Q) components by an orthogonal rotation matrix of size $4 \times 4$. With a $4 \mathrm{D}$ rotation, two different symbols are combined. Hence, the demapping complexity is drastically increased, compared to 2D. For this reason, 4D rotation was only adopted for QPSK in the DVB-NGH specification [14]. In this thesis, we only consider a $2 \mathrm{D}$ rotation with NURCs, since they require a very high demapping complexity compared with QPSK. For further information on 4D rotation, please refer to [25].

Fig. 3.19 shows two examples of 2D-NURCs optimized with the OBR method. On the left, a 16NURC optimized for a SNR target of $15 \mathrm{~dB}$, for i.i.d. Rayleigh channel, with a rotation angle of $18.5^{\circ}$. On the right, a 64 NURC optimized for $20 \mathrm{~dB}$, with a rotation angle of $12^{\circ}$. For this purpose, a CIL must be implemented after the rotation. For instance, when using a single RF channel, this could be achieved by a simple time shifting of the Q component (Q-delay, 


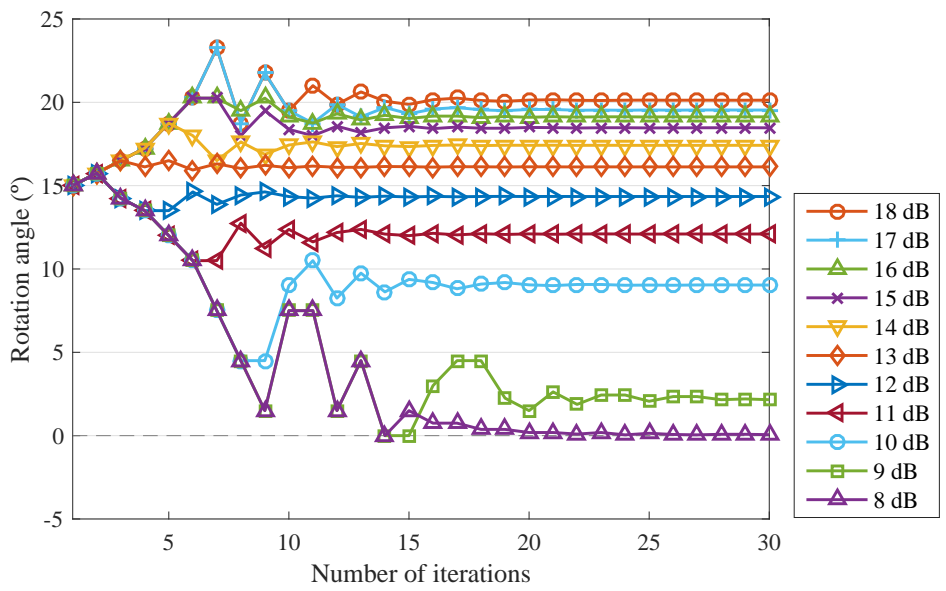

Figure 3.18: Rotation angle optimization process with method OBR and Nelder-Mead. 16NURC for a SNR range from 8 to $18 \mathrm{~dB}$.
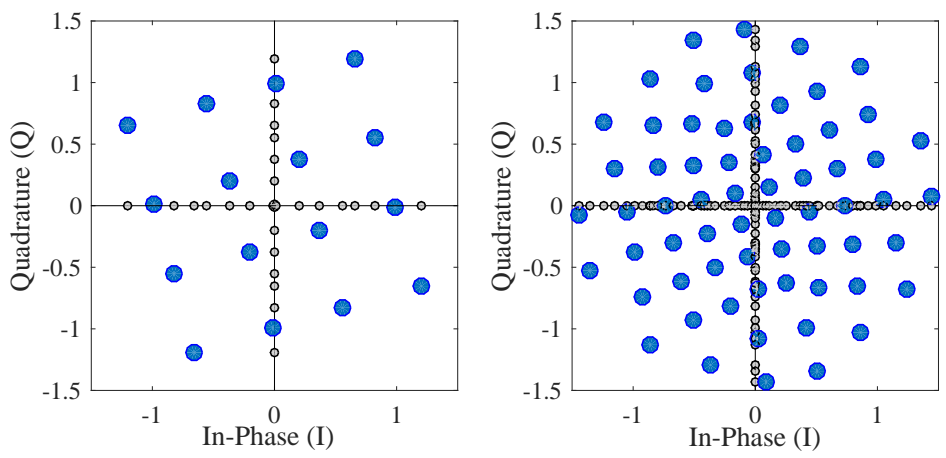

Figure 3.19: 16NURC and 64NURC optimized for i.i.d. Rayleigh channel and SNR targets of 15 and $20 \mathrm{~dB}$, with rotation angles of $18.5^{\circ}$ and $12^{\circ}$, respectively.

as specified in DVB-T2), so that the Q component of symbol $n$ is transmitted with the I component of the symbol $n+1$. This ensures each component of the same symbol experiences a different fading realization.

\subsubsection{Optimization with Additional Rotation}

Unlike OBR, with OAR the rotation angle is included as a new variable in the optimization process. The constellations need to be re-optimized taking this 


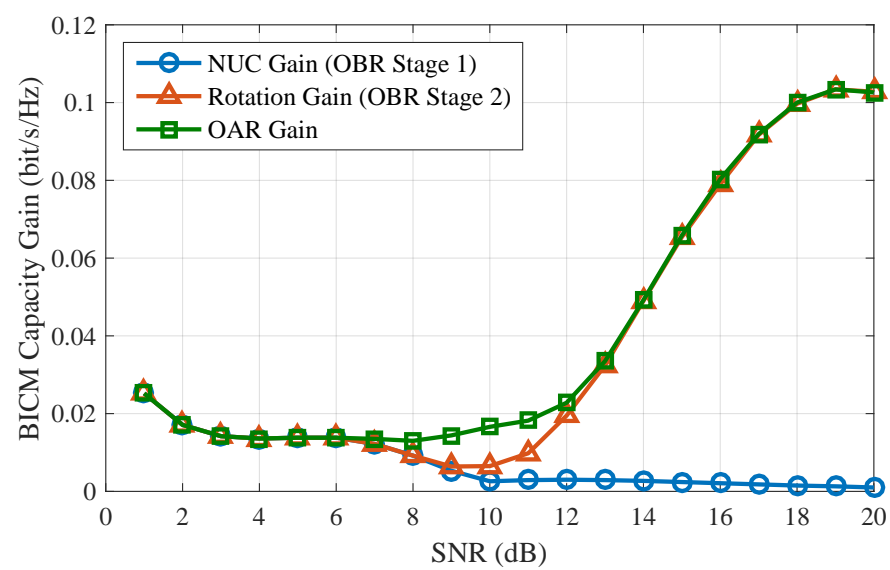

Figure 3.20: BICM capacity gain of 16NURC compared to non-rotated QAM, obtained with OBR and OAR methods for i.i.d. Rayleigh channel. The two contributions of OBR are shown by separate.

\begin{tabular}{|c|c|c|c|c|c|c|c|}
\hline Code Rate & $\mathbf{7 / 1 5}$ & $\mathbf{8 / 1 5}$ & $\mathbf{9 / 1 5}$ & $\mathbf{1 0 / 1 5}$ & $\mathbf{1 1 / 1 5}$ & $\mathbf{1 2 / 1 5}$ & $\mathbf{1 3 / 1 5}$ \\
\hline QPSK & 15.2 & 23.6 & 25.5 & 26.4 & 27.1 & 27.4 & 27.6 \\
\hline 16NURC & - & 8.4 & 10.3 & 14.4 & 16.1 & 18.4 & 19.5 \\
\hline 64NURC & - & - & - & - & 5.5 & 9.7 & 12.1 \\
\hline 256NURC & - & - & - & - & - & 7.5 & 10.2 \\
\hline
\end{tabular}

Table 3.2: Rotation angles $\left({ }^{\circ}\right)$ optimized for 2D-NURCs, for i.i.d Rayleigh channel.

new value into account, and the total number of DoF in this case is $\frac{M}{2}$. Compared to OBR, the optimization burden complexity only increases in a single DoF, and both stages are performed in a single optimization step. Moreover, the constellation symbols are optimized without capacity loss because of the rotation.

As an example, Fig. 3.20 shows the capacity gain achieved for 16 NURCs with both OBR and OAR methods, compared to non-rotated QAM constellations. Note that the two contributions of the OBR method are considered by separate, i.e., the NUC optimization (blue) and the following rotation (orange), while the only contribution of OAR is shown in gray. Observing Fig. 3.20, the maximum BICM capacity is significantly higher using the second method OAR, especially for medium SNRs, region where the rotation starts to provide a slight gain (range from 9 to $11 \mathrm{~dB}$ ). In addition, optimizing NURCs with OAR increases the SNR range where there is a gain with the rotation ( 7 and $8 \mathrm{~dB}$ ). Thus, NURCs optimized with the OAR method are used in following 


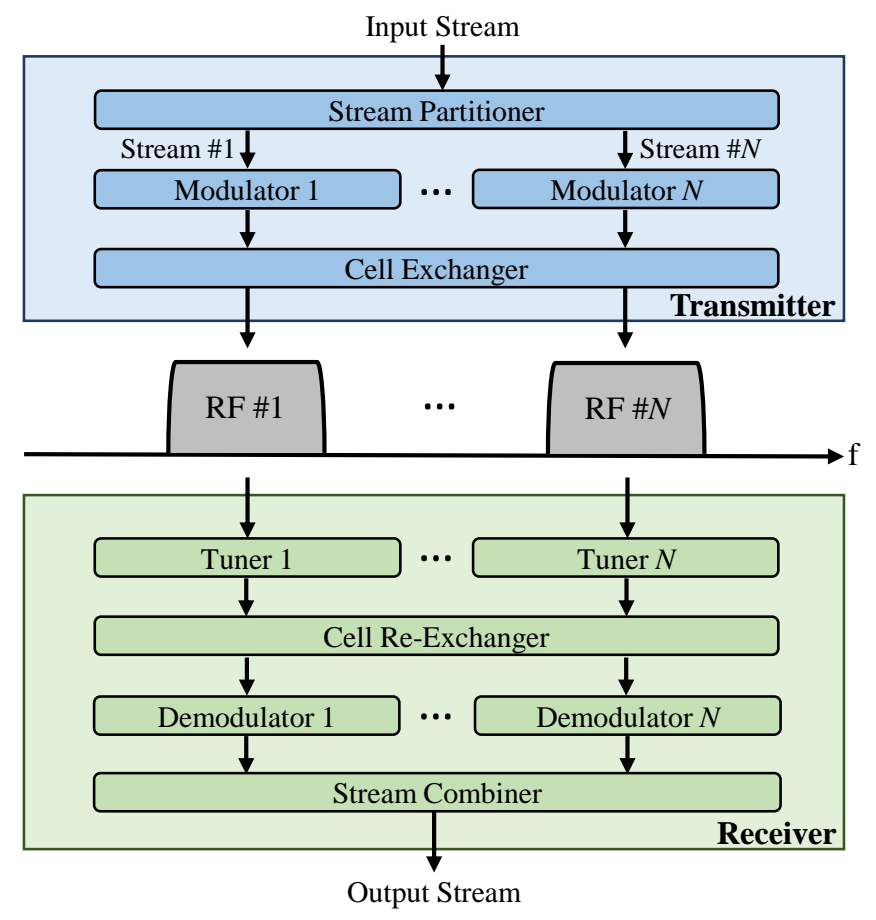

Figure 3.21: Channel Bonding concept extended to $N$ RF channels.

sections. Table 3.2 shows the resulting rotation angles, for CRs that provide a rotation gain $(7 / 15$ to $13 / 15)$ higher than $0.1 \mathrm{~dB}$. A hyphen indicates that the best option is not to apply the rotation, for the particular case of single-RF transmissions, since the gain obtained is almost negligible. As can be observed, the optimum rotation angle is higher for low-order constellations and high CRs. Rotation gains obtained using the selected angles are provided in next sections.

\subsection{Application of NURC to Multi-RF Tech- niques}

$\mathrm{CB}$ consists of the reception of data in parallel from two RF channels and enables doubling peak service data rate. There are two different operation modes. The basic mode is known as plain CB, in which reception is performed by means of two tuners. The second operation mode, known as SNR averaging, exploits 

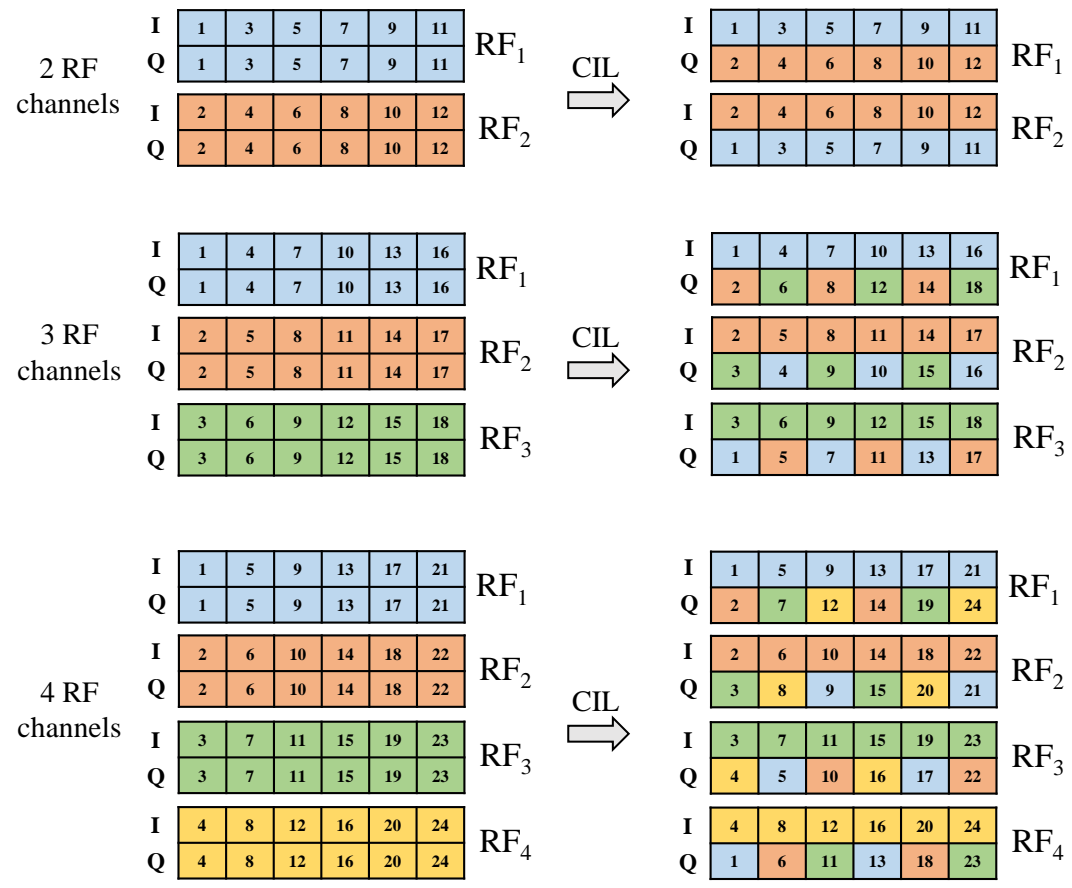

Figure 3.22: Component Interleaver designed for multi-RF techniques, using 2, 3 or $4 \mathrm{RF}$ channels. It is only applied to the $\mathrm{Q}$ component. The I component keeps the same distribution.

increased frequency diversity by means of frequency interleaving of the service data among two RF channels, thus improving transmission robustness [62]. On the other hand, TFS distributes the data of each service across multiple RF channels by means of time slicing. Data is received by means of frequency hopping [60]. With TFS, frequency interleaving is achieved by a time interleaving duration that covers the transmission over multiple RF channels. With CB and SNR averaging, a cell exchanger is used so that one half of data is sent over each RF channel, as Fig. 3.21 shows. The symbols are then received using an cell re-exchanger. Afterwards, each output signal has to be sent to a different demodulator stage, where decoding process takes place. In this thesis, we only consider CB with SNR averaging for potential combination with constellation rotation.

The main advantage of CB with SNR averaging is the increased RF performance. Studies in [105] reveal the typical signal PI between RF channels in the 
UHF (Ultra-High Frequency) band. In a single-RF transmission, the reception of the complete delivered set of services is limited by the RF channel with the lowest SNR. If one of the RF channels cannot be decoded, the services carried within are lost, regardless of better conditions of the other channels. With a cell exchanger, all services are received with a global SNR corresponding to an averaged SNR among the RF channels [59]. As a matter of considering the gain provided by CB or TFS $\left(G_{M u l t i-R F}\right)$, Eq. 3.2 accounts for the additional SNR margin provided by multi-RF $\left(S N R_{M u l t i-R F}\right)$ over the SNR of the worst received $\mathrm{RF}$ channel $\left(S N R_{\text {worst }}\right)$.

$$
G_{\text {Multi-RF}}[d B]=S N R_{M u l t i-R F}-S N R_{\text {worst }}
$$

Additional gains can be exploited by the combination of rotated constellation and multi-RF techniques. In such case, it is desirable that each component is transmitted in a different RF channel. In this thesis, we apply the CIL only to the $\mathrm{Q}$ component, and the design becomes different depending on the number of RF channels currently used in the transmission. It is based on the CIL specified in the DVB-NGH (Next-Generation Handheld) standard [14], since ATSC 3.0 does not implement this type of interleaving. The $\mathrm{Q}$ components are shifted from RF channel to RF channel as shown in Fig. 3.22.

\subsection{Performance Analysis Based on Physical La- yer Simulations}

In the following section, the gain of the different NUCs designed (with and without rotation) is presented in terms of SNR (dB), under AWGN and i.i.d. Rayleigh channels. Note that the design and implementation of these constellations only affect the mapping and demapping processes. For the rest of blocks implemented in both the transmitter and receiver, the ATSC 3.0 specification was used. An optimum ML demapper was employed in all cases, since the use of suboptimum demappers to reduce the complexity is explored in Chapter 4. A low-density parity-check (LDPC) code length of 64800 bits was used, with bit and frequency interleavers activated [15]. The Time Interleaver (TIL) is deactivated in this section, since no gain can be obtained under the conditions of channel models considered (entries of the channel matrixes are independent amongst each other in time and space, with zero-mean). Ideal channel estimation is also considered. The complete transmit to receive simulation chain is defined in Appendix A. Regarding the stopping criterion, for each SNR a maximum number of $10^{4}$ and a minimum number of $10^{3}$ TIL blocks are simulated. Finally, the selected quality of service for comparisons is a bit error rate Bit Error Rate (BER) of $10^{-4}$ after BCH decoding. 


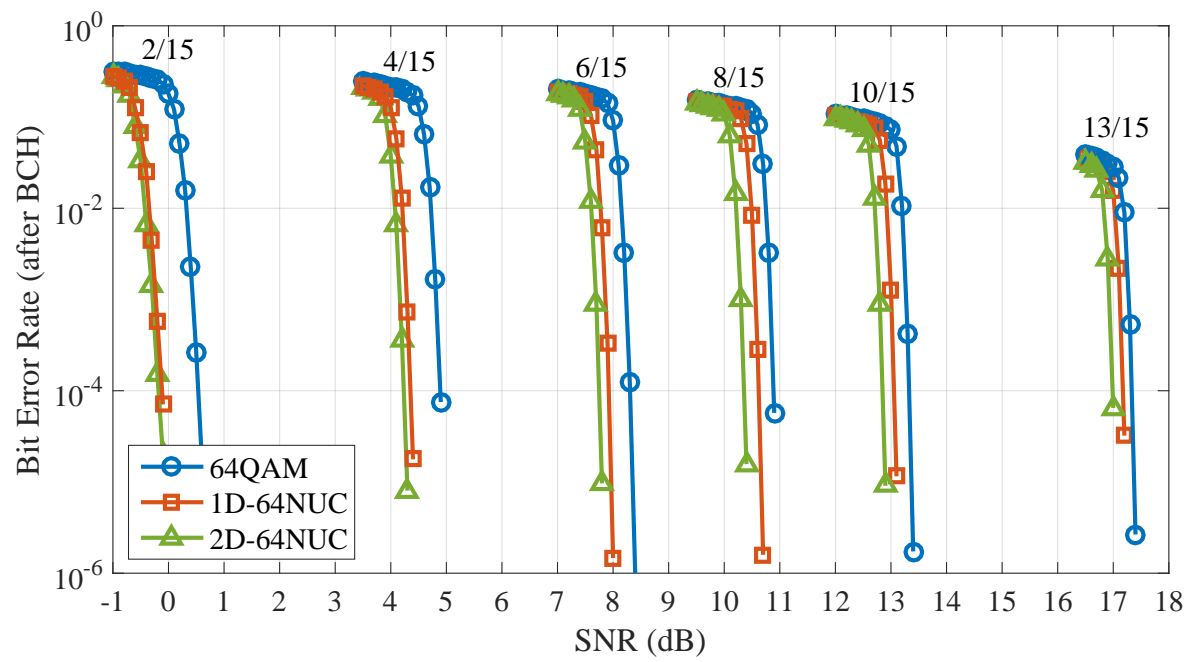

Figure 3.23: SNR Performance of different types of constellation: 64QAM, 1D-64NUC and 2D-64NUC, for different CRs and AWGN channel.

\subsubsection{Non-Uniform Constellations Gain}

Fig. 3.23 presents an example of the performance gain achieved using the 1D- and 2D-64NUCs optimized in Section 3.3, for AWGN channel and some representative CRs. On the other hand, Figs. 3.24 and 3.25 summarize the performance gains for all constellation orders and for AWGN and i.i.d. Rayleigh channels, respectively. Blue bars represent the SNR gain obtained with 1DNUCs, while yellow gains are obtained using 2D-NUCs. In general, three different conclusions can be formulated. First, the higher the constellation order, the larger the maximum gain achieved. Since the difference to the unconstrained Shannon limit with QAM constellations grows with the constellation order, there is more room for a possible optimization. Second, 2D-NUCs always obtain same or better gains than 1D-NUCs. A two-dimensional optimization permits the symbols a higher freedom to reach their optimum position (even they can evolve towards the squared shape), thus obtaining higher gains. Last but not least, we can affirm that, in general terms, medium CRs give the higher SNR gain. The only exception is given for 16QAM, where the best results are obtained for low CRs, due to the very small gap existent from QAM to the Shannon limit. In general, the gains obtained are very similar to those obtained in ATSC 3.0 [17], since we follow a similar approach and constellations are designed for the same channel models. 


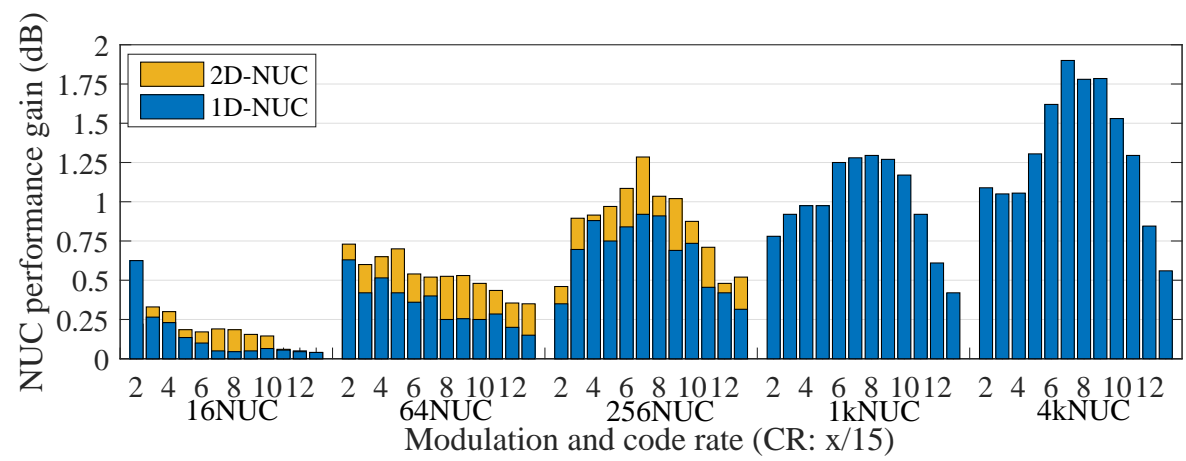

Figure 3.24: NUC Performance gain under AWGN channel. Constellations from 16 to 4096 symbols, CRs from $2 / 15$ to $13 / 15$.

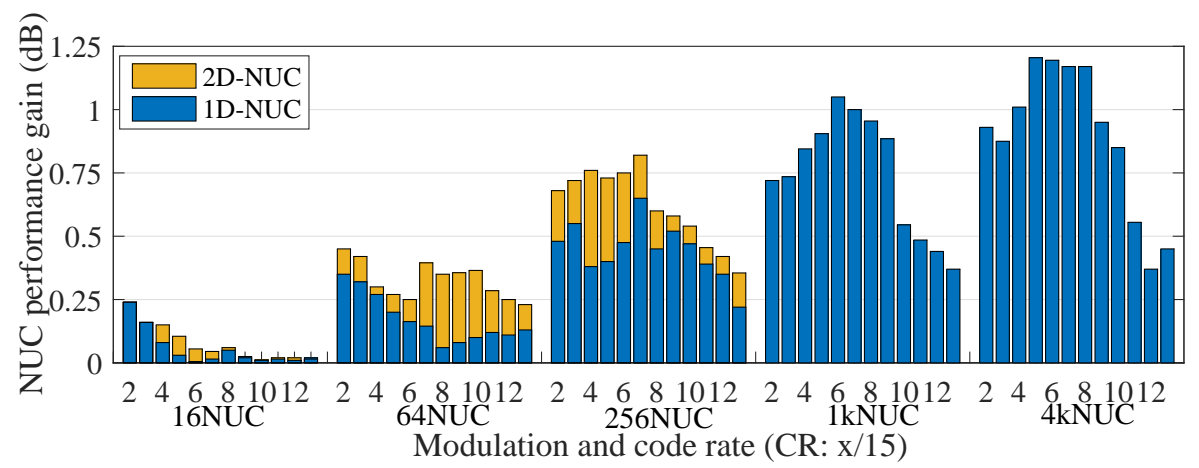

Figure 3.25: NUC Performance gain under i.i.d. Rayleigh channel. Constellations from 16 to 4096 symbols, CRs from 2/15 to $13 / 15$.

The SNR gains obtained for AWGN are, for all constellations and coding rates considered, higher than those obtained for i.i.d. Rayleigh. Maximum differences are obtained for high constellation orders and medium CRs, where higher gains are obtained. The difference in this case is up to $0.6 \mathrm{~dB}$. The use of a more realistic channel with fading statistics hampers the non-uniform constellations to approach the theoretical limit. Note that differences could be even higher if constellations were optimized only for AWGN channel, instead of the combination of both of them. Tables 3.3 and 3.4 show the relationship between each CR and SNR for which constellations were designed, for AWGN and i.i.d. Rayleigh channels, respectively. For the sake of simplicity, only even CRs are shown. 


\begin{tabular}{|c|c|c|c|c|c|c|}
\cline { 2 - 7 } \multicolumn{1}{c|}{} & \multicolumn{7}{c|}{ Code Rate } \\
\hline Constellation & $\mathbf{2 / 1 5}$ & $\mathbf{4 / 1 5}$ & $\mathbf{6 / 1 5}$ & $\mathbf{8 / 1 5}$ & $\mathbf{1 0 / 1 5}$ & $\mathbf{1 2 / 1 5}$ \\
\hline $\mathbf{1 6 N U C}$ & -3 & 2 & 5 & 7 & 9 & 11 \\
\hline $\mathbf{6 4 N U C}$ & 0 & 5 & 8 & 11 & 13 & 16 \\
\hline $\mathbf{2 5 6 N U C}$ & 2 & 7 & 11 & 14 & 17 & 21 \\
\hline 1kNUC & 4 & 9 & 14 & 18 & 21 & 25 \\
\hline 4kNUC & 5 & 11 & 16 & 21 & 26 & 30 \\
\hline
\end{tabular}

Table 3.3: SNR (dB) assumed for each constellation and CR combination, AWGN channel.

\begin{tabular}{|c|c|c|c|c|c|c|}
\cline { 2 - 7 } \multicolumn{1}{c|}{} & \multicolumn{7}{c|}{ Code Rate } \\
\hline Constellation & $\mathbf{2 / 1 5}$ & $\mathbf{4 / 1 5}$ & $\mathbf{6 / 1 5}$ & $\mathbf{8 / 1 5}$ & $\mathbf{1 0 / 1 5}$ & $\mathbf{1 2 / 1 5}$ \\
\hline $\mathbf{1 6 N U C}$ & -2 & 3 & 6 & 9 & 12 & 15 \\
\hline $\mathbf{6 4 N U C}$ & 1 & 6 & 10 & 13 & 16 & 20 \\
\hline $\mathbf{2 5 6 N U C}$ & 3 & 9 & 13 & 17 & 20 & 24 \\
\hline 1kNUC & 5 & 11 & 16 & 21 & 25 & 29 \\
\hline 4kNUC & 7 & 13 & 19 & 24 & 29 & 34 \\
\hline
\end{tabular}

Table 3.4: SNR (dB) assumed for each constellation and CR combination, i.i.d. Rayleigh channel.

\subsubsection{Rotation Gain}

Fig. 3.26 depicts the rotation gain, i.e. the improvement in the required SNR due to the constellation rotation. We consider the NURCs optimized with the OAR method, from 16 to 256 symbols, and also the QPSK constellation (without any possible optimization). The OBR method is not used in this section, since the capacity results obtained with OAR are always the same or better than those achieve with this method. As in previous section, LDPCs and BILs from ATSC 3.0 are used. The highest rotation gain is obtained for low-order constellations and high CRs. The additional diversity introduced by RCs improves the performance for higher CRs, whereas for lower CRs it is preferable to rely on the error-correction capabilities of the FEC code. In this case, a maximum gain of $1.7 \mathrm{~dB}$ is obtained using QPSK 13/15.

\subsubsection{Non-Uniform Rotated Constellations with Multiple RF Channels}

This section focuses on the gain of rotated constellations when making use of inter-RF frequency diversity, transmitting the I/Q components of each rotated symbol in different RF channels. First, we only consider 2 RF channels. The study is extended to $4 \mathrm{RF}$ channels. In order to model these RF channels, 


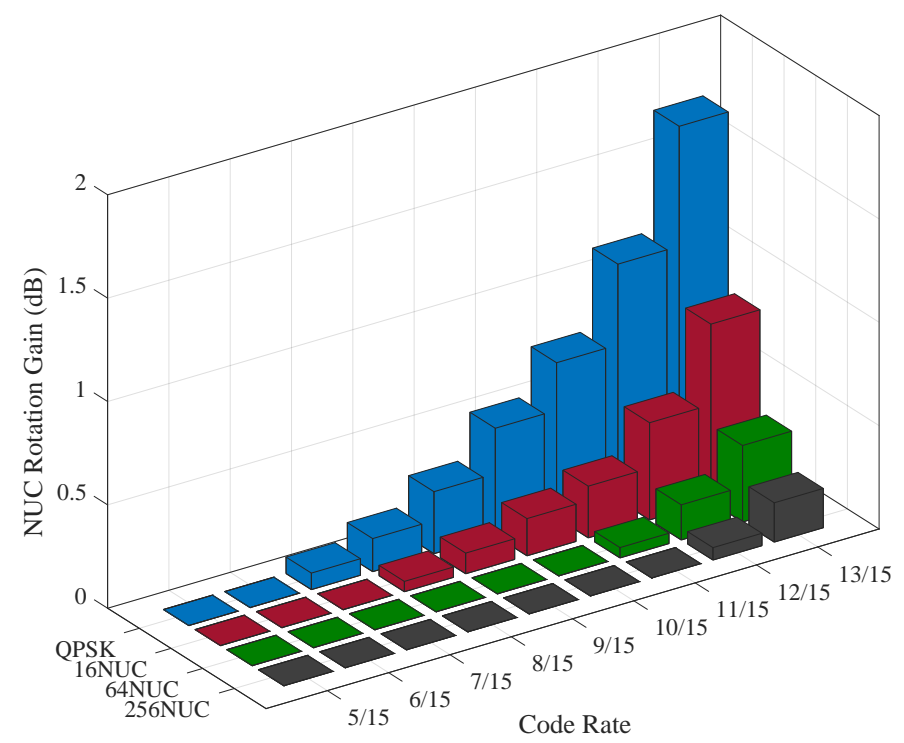

Figure 3.26: Rotation gain (dB) of 2D-NURCs designed with OAR for i.i.d. Rayleigh channel, when a Q-delay is applied. CRs from 5/15 to 13/15.

two different power imbalances are defined, i.e. 3 and $9 \mathrm{~dB}$. We consider $3 \mathrm{~dB}$ to simulate that the $\mathrm{RF}$ channels are close in the same frequency range, and transmitted under similar channel conditions. An imbalance of $9 \mathrm{~dB}$ is also studied as a worst-case scenario. Studies in [105] reveal that it is possible to find imbalances up to $10 \mathrm{~dB}$ in certain locations.

\section{Gain with 2 RF Channels}

Fig. 3.27 summarizes the multi-RF gain $\left(G_{m u l t i-R F}\right)$ obtained for all NUCs, optimized for a single RF transmission, and the two considered scenarios (imbalances of 3 and $9 \mathrm{~dB}$ ). The multi-RF gain is calculated using Eq. 3.2. It should be noted that the higher the PI between RF channels, the larger the gain. The gain also depends on two additional parameters: CR and order of constellation. Regardless of the PI between RF channels and without applying any rotation to the constellation, the highest gains are for very robust LDPC codes (low CR) with a low-order modulation, e.g. QPSK, obtaining a maximum gain of $5.9 \mathrm{~dB}$. As a half-way point, CRs such as $7 / 15$ or $8 / 15$ provide a gain which is approximately the half of the PI between RF channels. From 


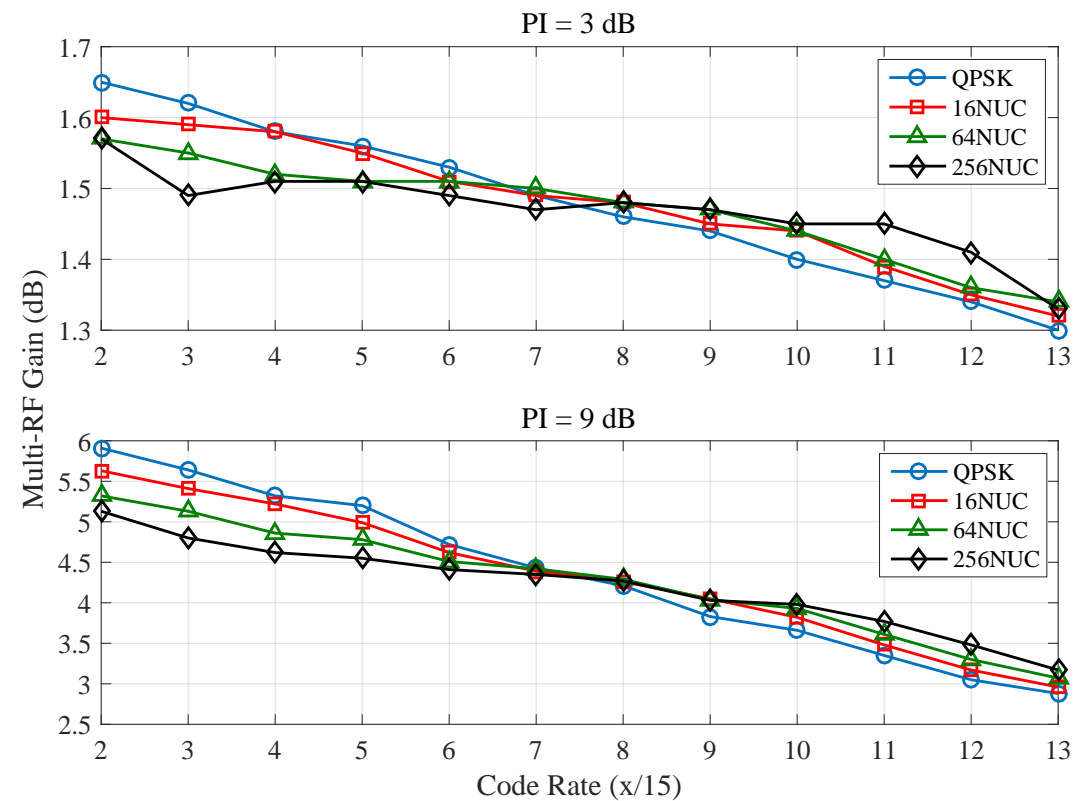

Figure 3.27: Multi-RF Gain (dB) for two RF channels, depending on the CR and modulation, i.i.d. Rayleigh channel. Two different scenarios are studied, i.e. PI of 3 and $9 \mathrm{~dB}$.

this point, we assume the worst considered scenario (9 $\mathrm{dB}$ of imbalance) for further results.

Fig. 3.28 shows the rotation gain $\left(G_{R O T}\right)$ of NURCs when using multi-RF techniques. As occurred with a single RF channel, the additional diversity introduced by RCs improves the SNR performance only for high CRs, but the range of CRs with additional gain is increased. In particular, there are two more combinations where a rotation gain can be achieved in multi-RF scenarios. These two combinations are QPSK with CRs 5/15 and 6/15, with rotation angles of $10.5^{\circ}$ and $12^{\circ}$ respectively. Moreover, the obtained gains become significantly higher. The maximum SNR gain, obtained with a QPSK constellation and CR $13 / 15$, is $3.9 \mathrm{~dB}$, which implies an increase of $2.2 \mathrm{~dB}$ compared to the single-RF case.

Fig. 3.29 depicts the total gain (continuous lines) achieved when combining both the rotation and multi-RF procedures $\left(G_{T}=G_{m u l t i-R F}+G_{R O T}\right)$, with a PI of $9 \mathrm{~dB}$, compared to the non-rotated case (dashed lines). In contrast to nonrotated constellations, the highest gain is achieved with the largest possible CR, when using the QPSK modulation. In this case, the SNR gain obtained is up 


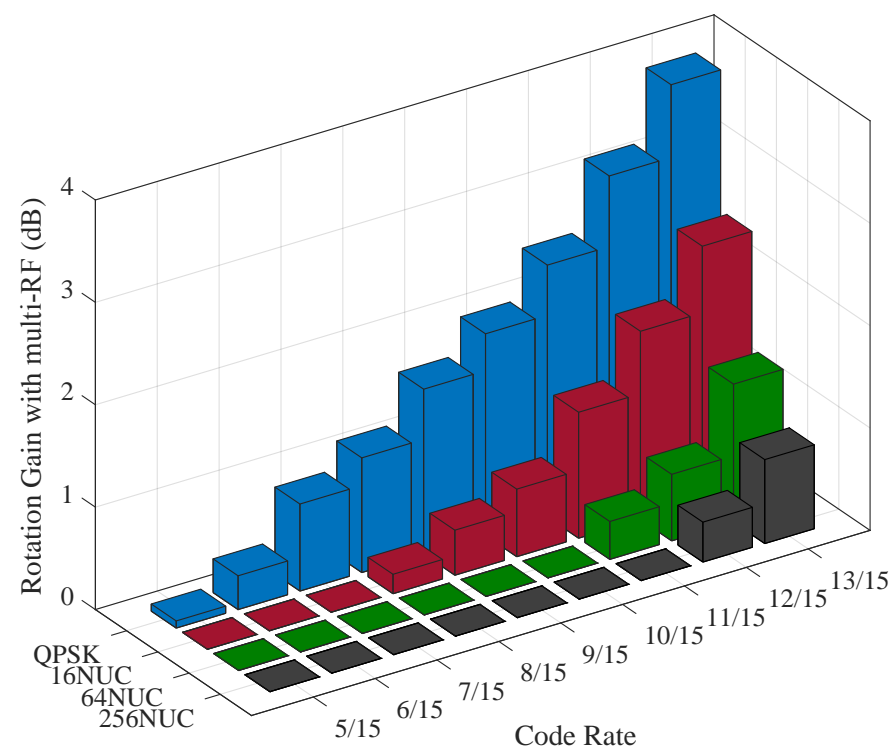

Figure 3.28: Rotation SNR gain $\left(G_{R O T}\right)$ of NURCs with multi-RF techniques and $2 \mathrm{RF}$ channels, for i.i.d Rayleigh channel and PI of $9 \mathrm{~dB}$.

to $6.7 \mathrm{~dB}$, from a total of $9 \mathrm{~dB}$ of imbalance. With higher-order constellations, even though the rotation provides a considerable gain, low CRs remain as the better option. The total gain $G_{T}$ is always over $4 \mathrm{~dB}$ for all cases. Thanks to the SNR averaging combined with the rotation, almost the half of power imbalance between RF channels could be recovered, for any configuration. To better explain the potential gains achieved, Fig. 3.30 shows an example of the performance obtained when considering $\mathrm{CB}$ and/or constellation rotation, for the particular case of a QPSK constellation and CR 13/15.

\section{Gain with 4 RF Channels}

In this section, the potential gain of NURCs with SNR averaging over 4 RF channels is analyzed. We consider 3 different scenarios, depending on the number of channels with a very low SNR compared to the best RF channel: when $1 / 4,2 / 4$ or $3 / 4 \mathrm{RF}$ channels are transmitted with a $\mathrm{PI}$ of $9 \mathrm{~dB}$, as shown in Fig. 3.31. From the diversity point of view, the case of $2 / 4 \mathrm{RF}$ channels is analog to $1 / 2$ with $2 \mathrm{RF}$ channels, studied in previous section.

From the two possible remaining scenarios, the most relevant result can be derived from the $3 / 4$ case, i.e. when $3 \mathrm{RF}$ channels present a low performance 


\subsection{Performance Analysis Based on Physical Layer Simulations}

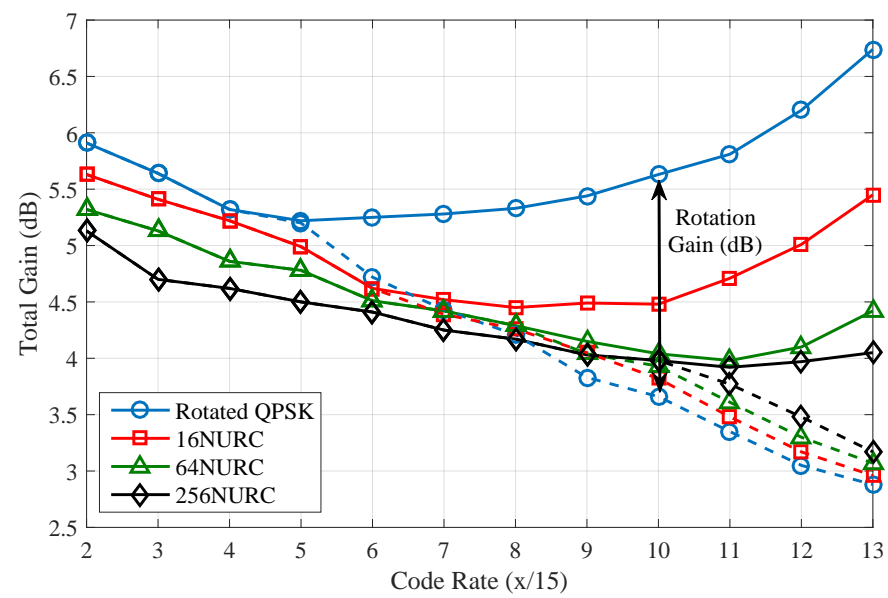

Figure 3.29: Total gain $\left(G_{T}\right)$ of NURCs with multi-RF techniques, for i.i.d Rayleigh channel and PI of $9 \mathrm{~dB}$ between the $2 \mathrm{RF}$ channels. Dashed lines represent the multi-RF gain $\left(G_{\text {multi-RF }}\right)$, without rotation.

compared to the best one. Thanks to multi-RF techniques and SNR averaging, the minimum required SNR can be drastically reduced, at the expense of requiring a higher SNR in the RF channel with the best performance. When rotated constellations are also employed, the SNR gain becomes higher especially with high CRs and low-order constellations, as occurred with $2 \mathrm{RF}$ channels (see the top of Fig. 3.32). For instance, with QPSK 13/15, a total gain of 3.8 $\mathrm{dB}$ is achieved in the 3 worst RF channels. Reducing the SNR performance in these 3 channels implies an increase of $5.1 \mathrm{~dB}$ in RF1. As expected, the SNR gain is lower than the $6.7 \mathrm{~dB}$ obtained with $2 \mathrm{RF}$ channels (equivalent to $2 / 4$ ).

On the other hand, with $1 / 4$ a single RF channel is transmitted with a SNR imbalance compared to the rest of channels. In this case, CB provides veryhigh gains, especially with low CRs. Without rotation, the maximum SNR gain obtained ranges between 7.5 and $7.8 \mathrm{~dB}$ (the maximum possible gain is 9 $\mathrm{dB}$ ), regardless of the constellation (see the bottom of Fig. 3.32). When using NURCs, the SNR range is increased (from 4/15 to 13/15), and the rotation gain becomes slightly higher, up to $3.2 \mathrm{~dB}$. Using QPSK 13/15, the total gain is $8.7 \mathrm{~dB}$, which means that the RF channel with poor performance is almost fully recovered, as long as the SNR of the rest of channels is maintained. 


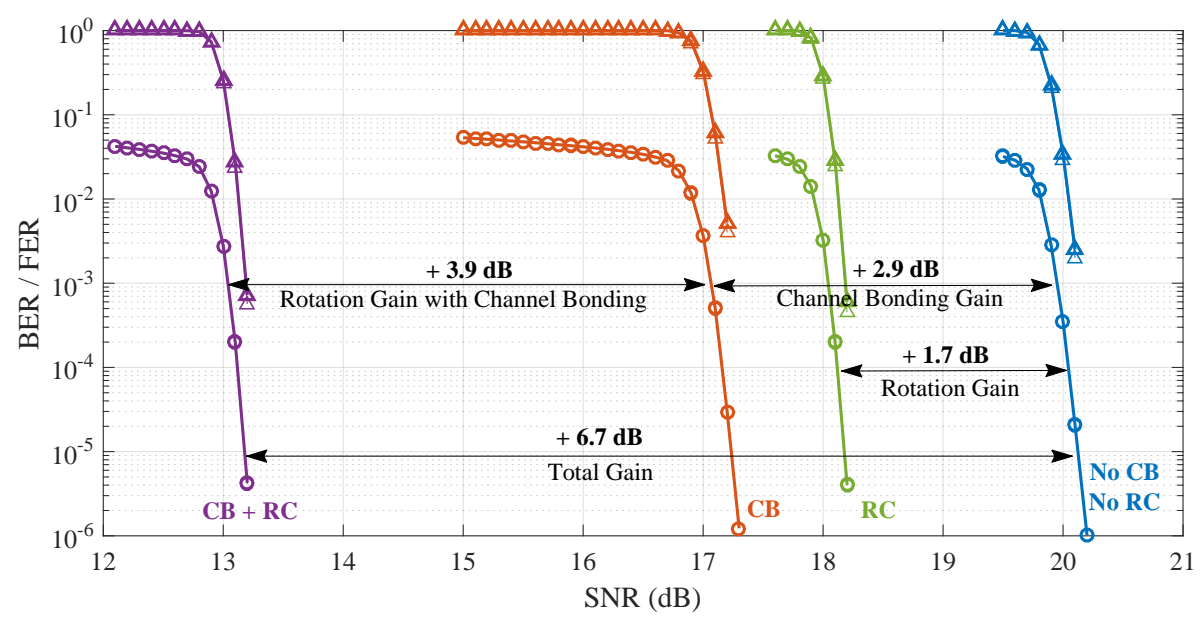

Figure 3.30: SNR performance with channel bonding and/or rotation. Two RF channels, for a QPSK CR 13/15 and i.i.d. Rayleigh channel, PI of $9 \mathrm{~dB}$.

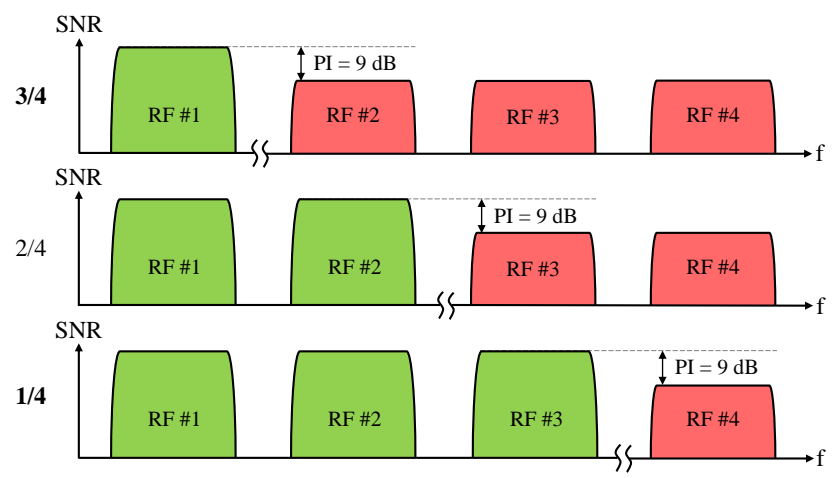

Figure 3.31: Three considered scenarios where multi-RF with SNR averaging and $4 \mathrm{RF}$ channels can be potentially beneficial. PI is fixed to $9 \mathrm{~dB}$.

\section{Gain with Erasures}

As an additional result, in this section we evaluate the potential gains when the RF channels present co-channel interferences that are simulated as erasures [28]. With erasures, there is a direct relationship between CR and the quality of the channels, which can be explained by considering the amount of erased information symbols during a co-channel interfered transmission. In other words, the presence of erasures limits the maximum CR for which error-free commu- 


\subsection{Performance Analysis Based on Physical Layer Simulations}
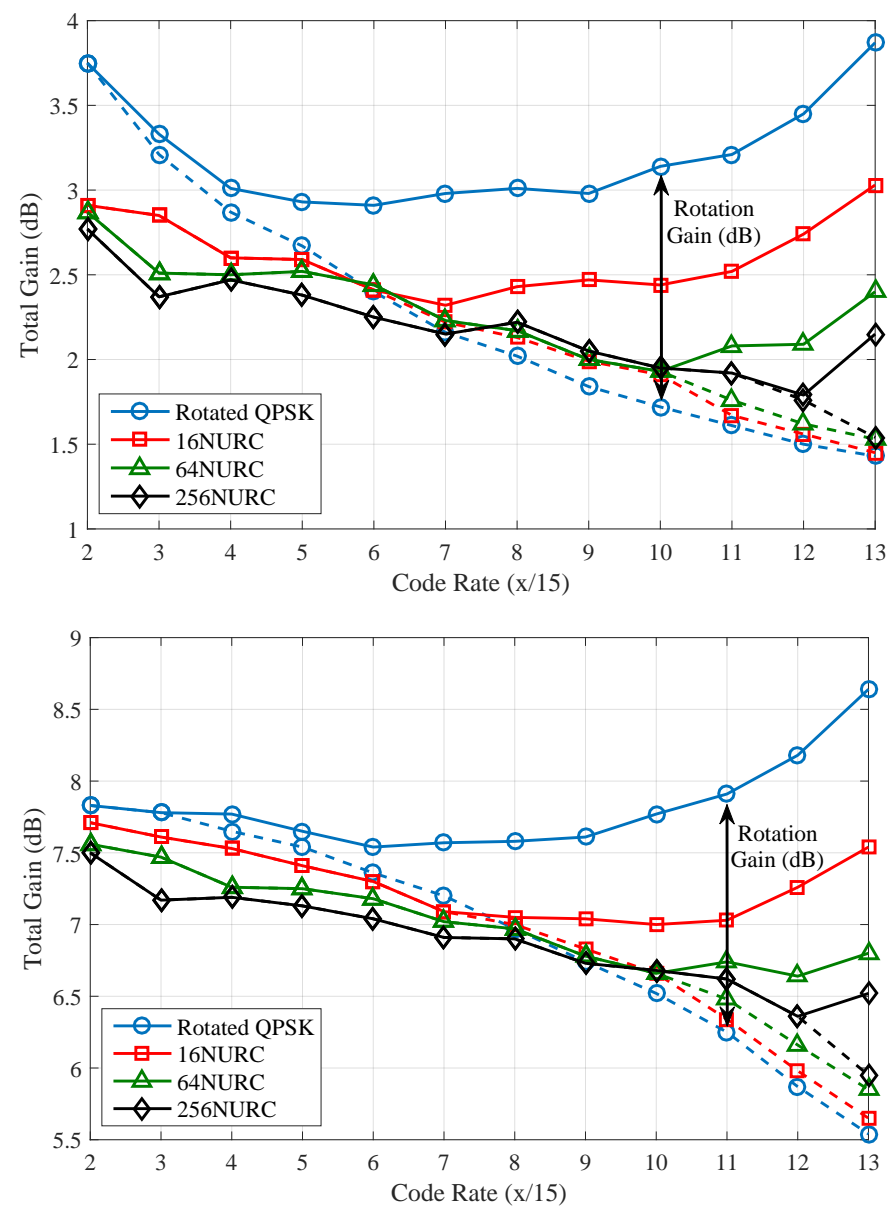

Figure 3.32: Total gain $\left(G_{T}\right)$ of NURCs with multi-RF and 4 RF channels, for i.i.d Rayleigh channel. 3/4 (top) and 1/4 (bottom) RF channels require higher a SNR. Dashed lines represent the multi-RF gain $\left(G_{C B}\right)$, without rotation.

nication is possible. A $25 \%, 50 \%$ and $75 \%$ of erasures require CRs lower than $3 / 4,1 / 2$ and $1 / 4$ respectively in order to repair the loss of information.

Fig. 3.33 depicts the minimum SNR required at the receiver when using 4 different RF channels, with SNR averaging and the presence of erasures in 1, 2 or 3 channels, without rotation (dashed lines). As mentioned before, the higher the CR, the larger the SNR required. With erasures and high CRs the SNR tends to infinite, which makes the demodulation process impossible. The use 


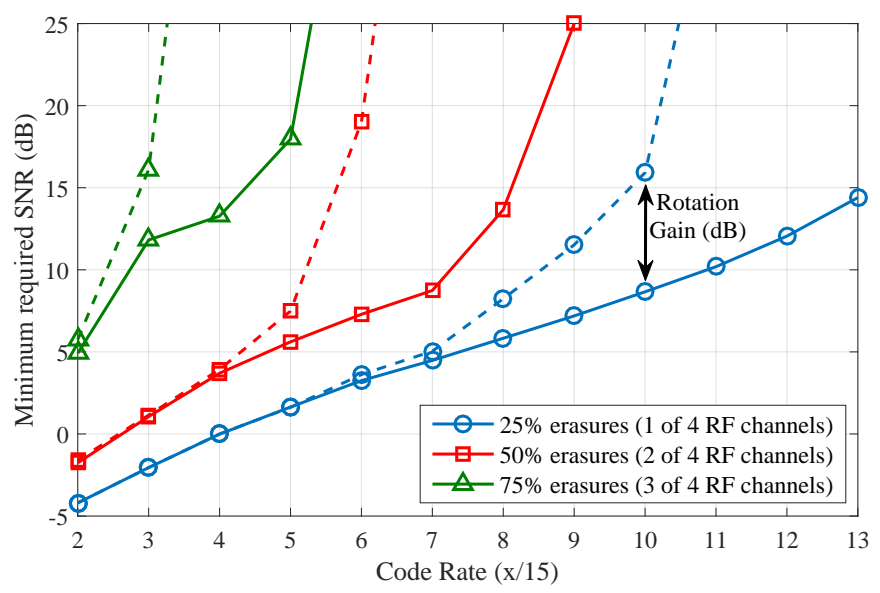

Figure 3.33: Minimum required SNR of rotated QPSK with multi-RF and erasures in the $25 \%, 50 \%$ or $75 \%$ of the 4 RF channels. Dashed lines represent the SNR required without rotation.

of $\mathrm{RC}$ (continuous lines) allows extending the SNR range where it is possible to recover the desired capacity. Whereas it was not possible to recover the capacity lost by the imbalance on RF2 with CRs higher than 1/2 for QPSK, with $\mathrm{RC}$ it is possible to recover the lost $\mathrm{RF}$ channel for higher SNR values, at the expense of a higher SNR requirement. With RCs, a $25 \%, 50 \%$ and $75 \%$ of erasures allow to use CRs lower than 13/15, 9/15 and 6/15, respectively.

\subsection{Conclusion}

In this chapter, different orders of one- and two-dimensional non-uniform constellations have been designed. Constellation symbols are optimized so the average BICM capacity is maximized, using the Nedel-Mead simplex algorithm [89]. The algorithm iteratively looks for the optimum constellation symbols, modifying their positions in the I/Q plane. In this thesis, $1 \mathrm{D}-\mathrm{NUCs}$ from 16 to 4096 symbols were designed, while 2D-NUCs were optimized up to 256 symbols. This assumption was also taken into account in the ATSC 3.0 standardization process [15]. The optimization of higher constellation orders is not feasible for the algorithm considered. The complexity of the optimization process is extremely high and the method does not find the optimum solution, reaching local minimums that do not provide the best result. 
1D-NUCs from 16 to 4096 symbols were designed, while 2D-NUCs were optimized up to 256 symbols. Results in this chapter show that the maximum BICM capacity gain obtained with $1 \mathrm{D}-\mathrm{NUCs}$ is $0.04,0.07,0.18,0.32$ and 0.43 $\mathrm{bit} / \mathrm{s} / \mathrm{Hz}$ with $16,64,256,1 \mathrm{k}$ and $4 \mathrm{kNUC}$, respectively. The gain is increased when using 2D-NUCs up to $0.05,0.1,0.22 \mathrm{bit} / \mathrm{s} / \mathrm{Hz}$ with 16,64 and $256 \mathrm{NUC}$. Naturally, there is a direct relation to the performance gain obtained, if the $\mathrm{CR}$ is accordingly selected taking into account the waterfall region of SNRs for which constellations were designed. The maximum performance gain achieved in i.i.d. Rayleigh channel with $1 \mathrm{D}-\mathrm{NUCs}$ is $0.05,0.32,0.65,1.05$ and $1.2 \mathrm{~dB}$ with 16, 64, 256, $1 \mathrm{k}$ and $4 \mathrm{kNUC}$, respectively. Regarding 2D-NUCs, the gains are increased to $0.16,0.45$ and $0.8 \mathrm{~dB}$. These gains are very similar to those obtained in the ATSC 3.0 standardization, since constellations are designed for the same channel models. Note that the use of AWGN and i.i.d. Rayleigh channel models has been considered in a single optimization. Selecting different channel models affects the final constellation, with symbols located on the intermediate positions obtained in separated optimizations. Constellations for two transmission modes, i.e. fixed and mobile receptions, were also investigated. Since both modes require totally different SNR values, the combination is not optimum. It is better to focus on a particular SNR. Another interesting concept is the use of condensed constellations. The idea is to transmit several symbols grouped in clusters, allowing to use more bits per symbol. In this case, the most significant bits provide similar robustness but the least significant bits are used to give additional information. On the other hand, the use of lowerorder constellations allows to increase the $\mathrm{CR}$, so in practice both constellations provide similar performance [34].

2D-NUCs can be rotated to further increase the gain at high SNRs. This chapter has first described the optimization process of Non-Uniform Rotated Constellations (NURC) and analyzed the performance in a single-RF transmission. Even though the demapping complexity is not increased, the SNR gain of NURCs in this case is not significant. The highest rotation gain is obtained for low-order constellations and high CRs, obtaining a maximum of $1.7 \mathrm{~dB}$ using QPSK 13/15. However, with multi-RF techniques such as Channel Bonding (CB) and Time-Frequency Slicing (TFS), the SNR gain is drastically increased, since I and Q components are transmitted in different RF channels. Hence, we have provided the multi-RF gain of NUCs with and without rotation. Without rotating NUCs, the higher the PI between RF channels, the larger the gain. The gain also depends on the $\mathrm{CR}$ and order of constellation. Regardless of the PI between RF channels, the highest gains are for very robust LDPC codes (low $\mathrm{CR}$ ) with a low-order modulation. When applying an additional rotation, the highest gain is achieved with the largest possible CR, when using the QPSK modulation. In this case, the SNR gain obtained is up to $6.7 \mathrm{~dB}$, with a PI of 
$9 \mathrm{~dB}$. When extending the results to $4 \mathrm{RF}$ channels, we have considered two additional scenarios: $3 / 4$ and $1 / 4 \mathrm{RF}$ channels with poor performance. The most important result derives from the second scenario, where the RF channel with poor performance obtains up to $8.7 \mathrm{~dB}$ of SNR gain using QPSK 13/15. Additional results to these scenarios have been provided, when the RF channels present co-channel interferences. A $25 \%, 50 \%$ and $75 \%$ of erasures require CRs lower than 3/4,1/2 and 1/4 respectively in order to repair the loss of information. With an additional rotation, the maximum $\mathrm{CR}$ is increased to $13 / 15,9 / 15$ and $6 / 15$, respectively. 


\section{Chapter 4}

\section{Low-Complexity Demapping and Quantization Algorithms}

The use of NUCs has different implications when implementing real receivers. Digital demodulators need to process all the received information, in order to retrieve the data that originally was transmitted. A wide range of techniques such as demodulation, bit de-interleaving or decoding needs to be performed. Most of these techniques have been widely studied in the current literature, but the use of non-uniform constellations affects some of them when retrieving the original data. The first technique is demapping. With NUCs, optimum demappers calculate the distances from the received signal to all constellation symbols in a different manner, since the shape of the constellation changes. In that sense, Section 4.1 analyzes the demapping complexity, while in Section 4.2 we propose a new low-complexity demapping algorithm that reduces the amount of distances to calculate the LLRs [104]. As an example of application, we provide the results for NUCs optimized in Chapter 3. The section focuses on the two-dimensional $256 \mathrm{NUC}$ constellations, which is the highest modulation order of 2D-NUCs optimized in this thesis, also considered in the ATSC 3.0 specification [15]. Section 4.3 provides performance results for code rates from $2 / 15$ to $13 / 15$.

Another study identified is signal quantization. The use of NUCs affects the quantization process, since constellation symbols and therefore LLR values are different from those obtained with QAM. Two possibilities are explored in Section 4.4, i.e. quantization of I/Q components and LLRs in the time de- 


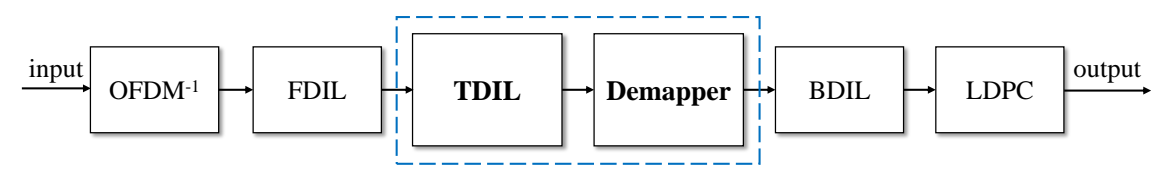

Figure 4.1: General receiver architecture. Time de-interleaving and demapping blocks are explored in this chapter.

interleaver, which needs the highest memory requirements. In this section, a low-complexity quantization method is also proposed. Performance results and memory requirements are presented in Section 4.5. The main findings of this chapter are summarized in Section 4.6.

\subsection{Demapping Complexity at the Receiver}

It is necessary to differentiate between two elements when comparing demapping complexity: the number of required distances to calculate each LLR and the complexity for the calculation of the distance itself. That is, the metric is calculated with a different dimensionality for $1 \mathrm{D}$ and $2 \mathrm{D}$ demapping. From the received symbol $y$, and the channel estimate $h$, each LLR $\Lambda_{l}$ is computed for all code bits $c_{l}, l=1, \ldots, B$, with $B$ as the number of bits that affect each dimension of a constellation. $B$ does not refer to the number of bits per symbol, also expressed as bits per cell $(b p c)$. For the LLR computation with the optimum ML demapper, a total number of $N$ Euclidean distances between the received symbol $y$ and all constellation symbols $x$ is calculated. A single output $\Lambda_{l}$ can be computed as in Eq. 4.1. Note that this expression was already introduced in Chapter 2 (Section 2.3).

$$
\Lambda_{l} \triangleq \log \frac{p\left(c_{l}=1 \mid y, h\right)}{p\left(c_{l}=0 \mid y, h\right)}=\log \frac{\sum_{x \in \chi_{l}^{1}} \exp \left(-\frac{|y-h x|^{2}}{\sigma^{2}}\right)}{\sum_{x \in \chi_{l}^{0}} \exp \left(-\frac{|y-h x|^{2}}{\sigma^{2}}\right)}
$$

where $\sigma^{2}$ represents the noise variance, $y$ is the received symbol, $x$ is a possible transmitted symbol, $h$ is the channel fading coefficient, and log refers to the natural logarithm. $\chi_{l}^{1}$ and $\chi_{l}^{0}$ denote the complementary sets of transmit symbols for which $c_{l}=1$ and $c_{l}=0$ respectively. The amount of complementary sets $\chi_{l}^{1}$ and $\chi_{l}^{0}$ has to be $B$. A ML optimum demapper has therefore to consider $2^{B}$ symbols in a $D$-dimensional (real-valued) space. Hence, the complexity $Q$ can be computed as follows: 
4.1 Demapping Complexity at the Receiver

\begin{tabular}{|l|c|c|c|c|c|}
\hline Constellation symbols & $\mathbf{1 6}$ & $\mathbf{6 4}$ & $\mathbf{2 5 6}$ & $\mathbf{1 0 2 4}$ & $\mathbf{4 0 9 6}$ \\
\hline QAM & 4 & 8 & 16 & 32 & 64 \\
\hline 1D-NUC & 4 & 8 & 16 & 32 & 64 \\
\hline 2D-NUC & 32 & 128 & 512 & 2048 & 8192 \\
\hline RC & 32 & 128 & 512 & 2048 & 8192 \\
\hline
\end{tabular}

Table 4.1: Demapping complexity of uniform QAM, 1D-NUC, 2D-NUC and RC, for different bits per cell, in terms of mathematical operations to compute the LLRs.

$$
Q=\mathcal{O}\left(D \cdot 2^{B}\right)
$$

where $\mathcal{O}$ represents the complexity order. It is also clear that the complexity depends on the type of constellation. Uniform QAM constellations can be split into two PAM constellations. Therefore, the demapper has to consider the half of symbols $\left(B=\frac{b p c}{2}\right)$ in one dimension $(D=1)$. With 1D-NUCs, the onedimensional demapping is also possible, and the complexity is maintained. In both cases, the complexity is calculated as:

$$
Q_{\mathrm{QAM}}=Q_{1 \mathrm{D}}=\mathcal{O}\left(2^{\frac{b p c}{2}}\right)
$$

On the other hand, with 2D-NUCs the demapper has to evaluate for all symbols $(B=b p c)$, and the distances in two dimensions (real and imaginary parts, $D=2$ ) need to be calculated. The same occurs with RCs, where the binary information is transmitted simultaneously in different I and Q components. The demapper has to consider all symbols in two dimensions, regardless of the constellations shape. With RCs, it is not possible to use a 1D-demapper in any case. Therefore, the complexity with rotated constellations is maintained. The complexity in both options is given by Eq. 4.4 .

$$
Q_{2 \mathrm{D}}=Q_{\mathrm{RC}}=\mathcal{O}\left(2^{b p c+1}\right)
$$

Table 4.1 presents the demapping complexity in terms of mathematical operations to compute the LLRs, depending on the type and order of constellation. The complexity order with $2 \mathrm{D}-\mathrm{NUCs}$ becomes especially high from $b p c=8$. The proposed demapping algorithm reduces the number of distances $N$ necessary to compute each LLR with this type of constellations, and it is explained next. 


\subsection{Low-Complexity Demapping Algorithm}

The proposed algorithm to demodulate 2D-NUCs is based on two different strategies. The first one is called Quadrant Search Reduction (QSR) and computes the LLRs discarding those distances with low probability of being computed. It takes advantage of the symmetry that constellations provide, selecting a cluster of points to compute the LLRs. It is based on the demappers proposed in [53] and [54] for RCs. The concept is similar to the sphere demapper [56], which also selects a cluster of points to compute the LLRs. From the received point, SD only selects the constellation points that are inside a fixed radius. The second strategy is called Condensed Symbols Reduction (CSR), and exploits the condensation of NUCs, especially at low CRs where some of the constellation symbols almost repeat the same position in the I/Q plane. It omits the computation of those distances which are similar to others already calculated, and replicates them. Both strategies can be combined in order to reduce from $69 \%$ to $93 \%$ the number of required distances, depending on the CR, with almost no performance loss compared to the optimal ML demapper. The proposed demapper can be combined with RCs, in order to improve the SNR requirement for high CRs with no additional complexity.

\subsubsection{Quadrant Search Reduction (QSR)}

The first strategy discards those distances that provide scarce information to the LLR computation. In this section, we apply QSR for 2D-NUCs, but the QSR algorithm can be used with any quadrant-symmetric constellation. The algorithm is divided into two main steps. The first step consists of calculating the probability of each symbol received, when they are transmitted from a particular quadrant of the constellation. The probability (from 0 to 1 ) of receiving a particular constellation symbol is calculated as the number of times the symbol is received divided by the total number of transmitted symbols. Then, the received symbols are organized by probability order. A total number of $10^{9}$ points is transmitted, over an i.i.d. Rayleigh channel. This number is confirmed in [54], and provides enough accuracy in the calculations. The rest of quadrants can be derived by symmetry. This step can be done off-line, storing the symbols by probability order in a look-up table. Then, the algorithm can compute the distances to the most probable symbols $N$ when necessary.

Fig. 4.2 shows two examples of the histograms achieved for 2D-256NUC in the first stage, for CRs $2 / 15$ and 13/15, designed in i.i.d Rayleigh channel for SNRs of 3 and $26 \mathrm{~dB}$ respectively. Both histograms have been obtained for the first quadrant (Q1). Note that in the figure, each symbol is represented in the horizontal axis using the integer number. For example, a symbol located in Q1 

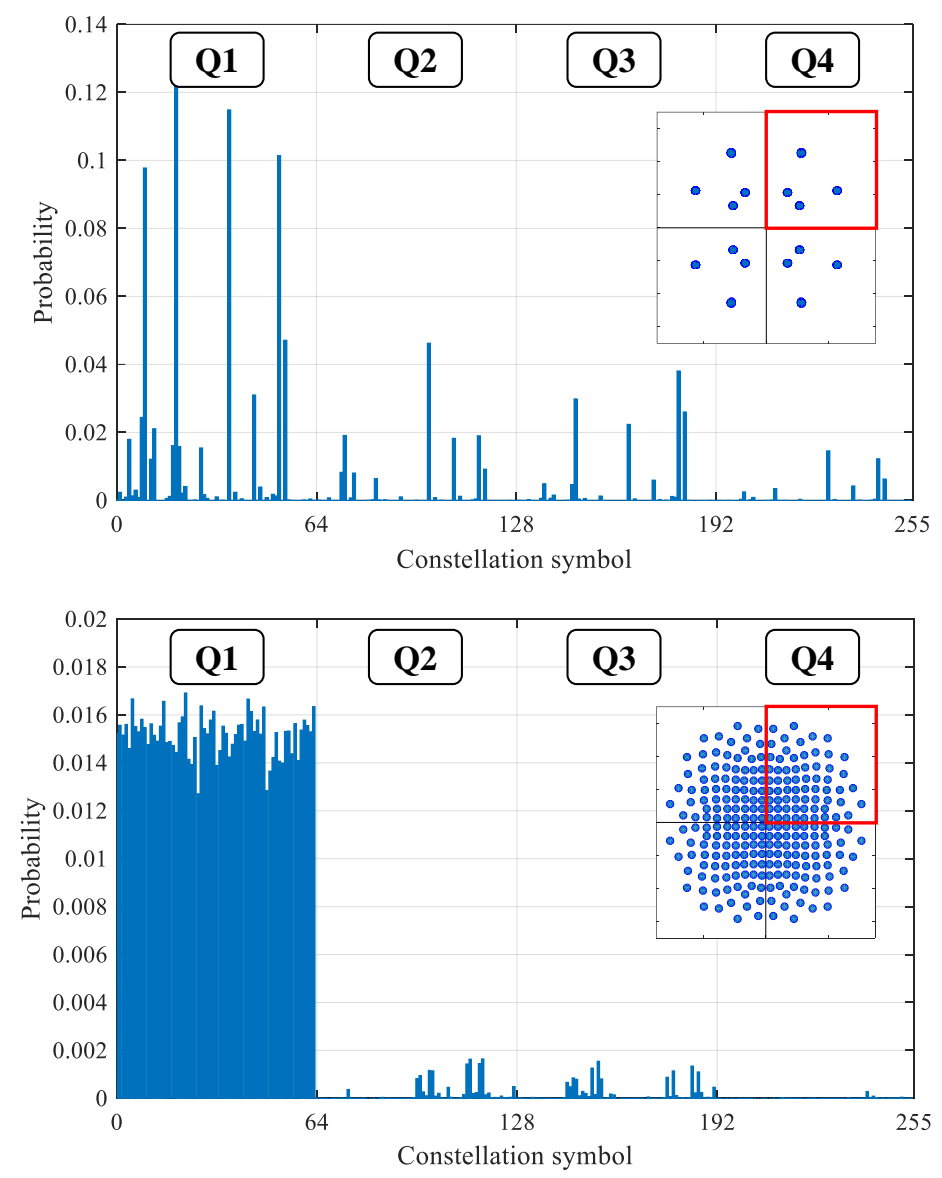

Figure 4.2: Histogram of the received constellation symbols for 2D-256NUC in i.i.d. Rayleigh channel, with code rates $2 / 15$ (top) and 13/15 (bottom).

with binary value 00110100 is directly related to the result given in position 52 . For a given CR, the transmission is done for the threshold SNR that provides a bit error rate (BER) of $10^{-4}$. As Fig. 4.2 shows, the probability of receiving a constellation point from the Q1 is higher for high SNRs (bottom). On the other hand, at low SNRs (top) and because of the high noise level, constellation points from other quadrants (especially Q2 and Q3) are received with a higher probability.

In a second step, the minimum number of distances with significant probability, necessary to implement the algorithm, is obtained. In order to select the 


\section{CHAPTER 4. LOW-COMPLEXITY DEMAPPING AND QUANTIZATION ALGORITHMS}

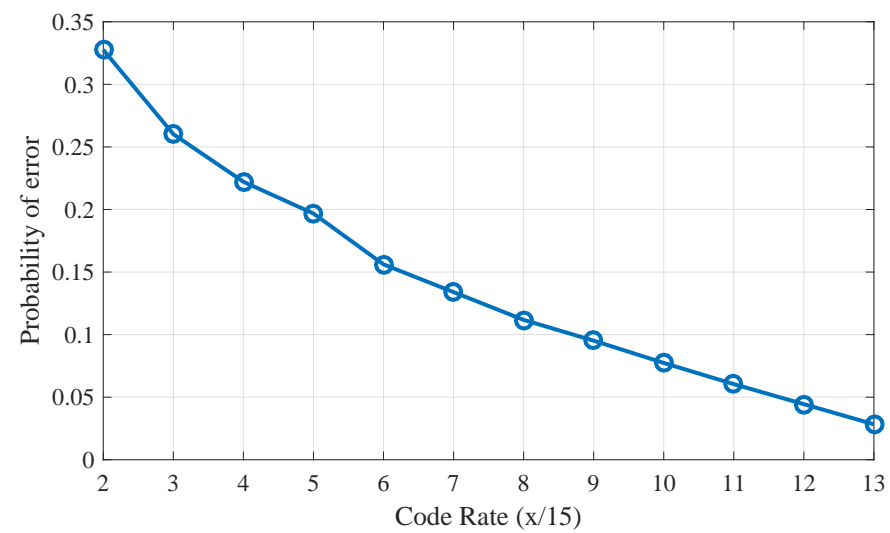

Figure 4.3: Probability of selecting an erroneous quadrant $\left(p_{e}\right)$ with 2D-256NUCs in i.i.d. Rayleigh channel, with code rates from $2 / 15$ to $13 / 15$.

final subset $N$, it is necessary to observe its impact on the system performance through a BER analysis. The smaller the number of distances $N$ considered, the worse the performance, but with a lower complexity. The proposed criteria is to select the smallest possible $N$ that ensures a performance loss smaller than $0.1 \mathrm{~dB}$.

With QSR, it is assumed that a symbol is received in the same quadrant that was transmitted. Hence, it is necessary to know the transmitted quadrant. The quadrant is determined using the sign of the $\mathrm{I} / \mathrm{Q}$ components of the received symbol. Let define the variable $p_{e}$ as the probability of selecting an erroneous quadrant. The constellation symbols that are in the edge of each quadrant provide less reliable results, and they usually lead to errors. The probability $p_{e}$ is higher for low CRs, with higher levels of noise power, as Fig. 4.3 shows. However, precisely those constellations with a very high probability of obtaining an erroneous quadrant suffer a significant condensation. The second strategy, which is explained next, takes advantage of this condensation and calculates only distances for symbols that are not repeated.

\subsubsection{Condensed Symbols Reduction (CSR)}

At low CRs, NUCs converge to lower orders of constellation. In this case, the constellation symbols share the MSB, maximizing the Euclidean distance and maintaining independent dimensions for each bit, while the LSB have almost no impact in the LLR computation. The information provided by the LSB is close to zero and will remain so, as long as the SNR value is sufficiently low. 

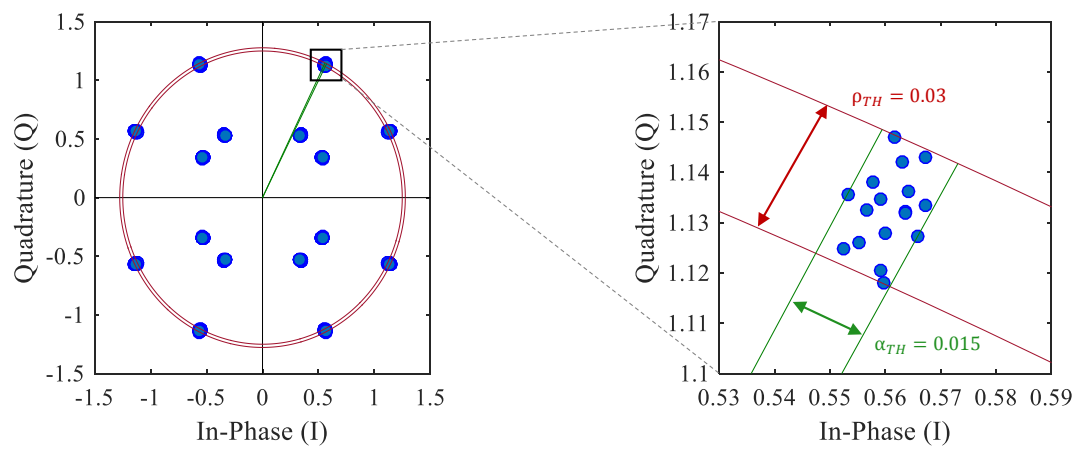

Figure 4.4: I/Q diagram of a 256NUC optimized for a CR 2/15 (left), with a zoom to one of the clusters detected by CSR (right), in which 16 symbols are grouped into one cluster.

As mentioned in other chapters, these constellations are called condensed constellations. They collapse to lower orders and symbols are grouped in clusters. Using this condensation, it is possible to transmit more bits per symbol. As main drawback, lower coding rates need to be used to keep the same spectral efficiency, so in practice both constellations provide similar performance [34].

For example, with a 2D-256NUC designed for a CR 2/15, which can be seen in the upper part of Fig. 4.2, only sixteen symbols are apparently visible, resembling a 16NUC. However, there are sixteen clusters with sixteen constellation symbols in almost identical positions. The CSR algorithm is based on this condensation and calculates a single distance for a complete cluster of symbols. The rest of distances are derived by replicating those previously calculated. In order to determine which symbols are grouped together and which not, it is necessary to define a minimum gap between them. The higher the gap selected, the bigger the number of symbols grouped together in a single distance to compute. This step introduces an insignificant loss in performance, since lineal Euclidean distances among condensed symbols in normalized NUCs are almost negligible, lower than $10^{-2}$. In order to detect clusters, an array $L$ with polar coordinates (radius and angle) is defined, for each constellation symbol. The algorithm evaluates each element in $L$ individually. The difference is obtained for two threshold variables, $\rho_{T H}$ (radius) and $\alpha_{T H}$ (angle in radians). If the difference is lower in both cases, a cluster is detected. Then, all remaining symbols fulfilling these criteria are removed from $L$, leaving the evaluated symbol as the representative one. It was observed through simulations that the selected symbol hardly affects the final performance. This process is repeated until reaching the end of the array $L$. Simulations have shown that 0.05 and 


\section{CHAPTER 4. LOW-COMPLEXITY DEMAPPING AND QUANTIZATION ALGORITHMS}

0.03 are suitable values for $\rho_{T H}$ and $\alpha_{T H}$ respectively. $L$ is then converted back to Cartesian coordinates and the remaining constellation symbols are stored. As an example, Fig. 4.4 depicts a 256 NUC optimized for a CR 2/15. Looking at the left side, it seems that only 4 constellation symbols are transmitted in the first quadrant. However, thanks to a deep zoom it is possible to distinguish up to 16 different symbols in a single position. As Fig. 4.4 shows, the maximum difference in $\rho_{T H}$ among all symbols is 0.03 , while the maximum angle is just 0.015. Thus, all symbols would be grouped inside the same cluster, following the process described above.

\subsubsection{Quadrant Condensed Search Reduction (QCSR)}

It is possible to combine both QSR and CSR into a single algorithm. We call this algorithm Quadrant Condensed Search Reduction (QCSR). The algorithm consists of taking the $N$ constellation symbols obtained with QSR and group them together as done with CSR. At low SNRs, the CSR algorithm is more dominant because the optimized 2D-NUCs are condensed, which also complicates the quadrant search. At high SNRs the noise impact is lower and the constellations are not condensed, and hence the QSR algorithm is dominant. A complete description is given in Algorithm 1.

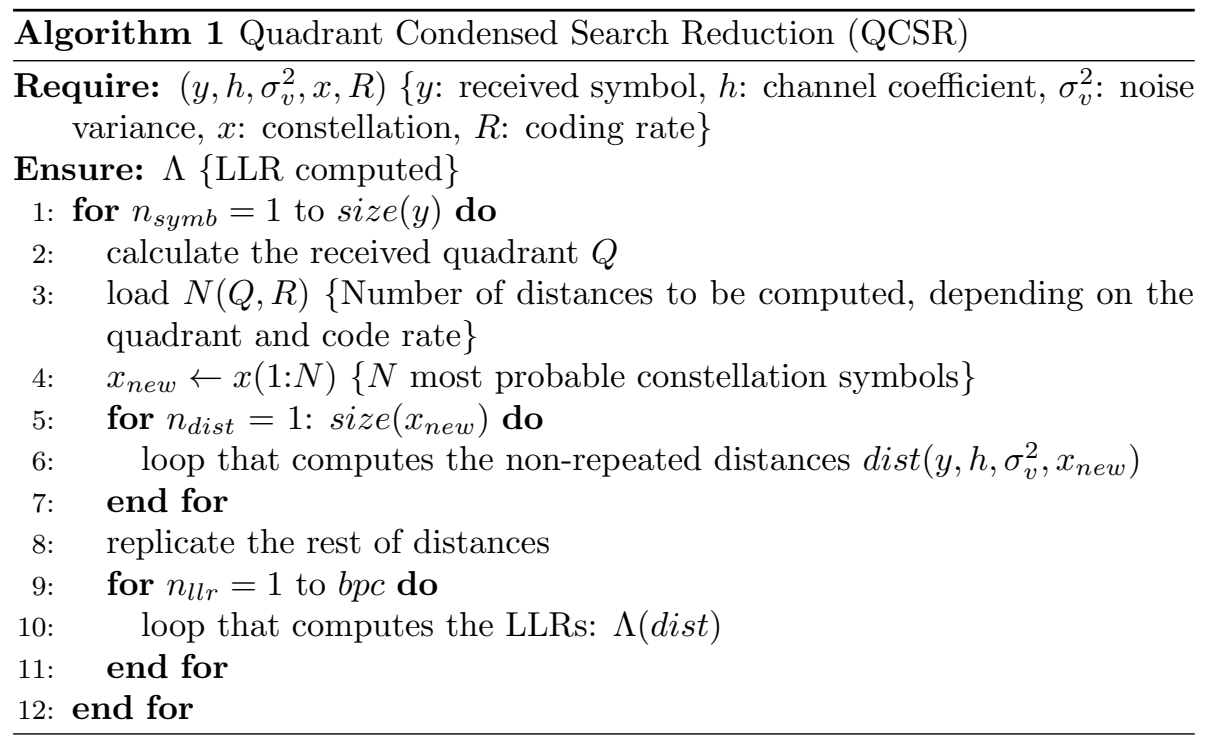


In the proposed algorithm, $y$ is the received symbol, $h$ is the channel fading coefficient, $\sigma_{v}^{2}$ represents the noise variance, $x$ is a possible transmitted symbol, and $R$ refers to the coding rate (from 0 to 1 ). $\Lambda$ denotes each LLR computed.

\subsection{Performance Evaluation: Minimum Num- ber of Distances}

This section compares first, in terms of BER performance, the results obtained with the optimum ML and the proposed QCSR demapper, providing the minimum number of $N$ distances that need to be calculated, for i.i.d. Rayleigh channel. Afterwards, the performance loss obtained for different channel models is shown. In the simulations, 2D-NUCs optimized in Chapter 3 are used. We also use bit-interleavers from ATSC 3.0 [15], with a LDPC code length of 64800 bits and FEC codes from 2/15 to $13 / 15$. No time and frequency interleavers are applied in this case.

\subsubsection{Calculation of the Minimum Number of Distances}

We define $N_{Q S R}, N_{C S R}$ and $N_{Q C S R}$ as the number of distances necessary for the QSR, CSR and QCSR demappers, respectively. As an example, Fig. 4.5 shows the performance of the proposed strategies QSR and CSR for 2D-256NUCs and CR 6/15, depending on the number of distances $N$. The selected criterion is to compare the SNR of each option that provides a BER of $10^{-4}$, selecting the smallest possible $N$ that ensures a performance loss smaller than $0.1 \mathrm{~dB}$. For this particular CR of $6 / 15$, both strategies QSR and CSR provide a reasonable performance degradation compared to ML, using a number of distances $N_{Q S R}=135$ and $N_{C S R}=88$ (selected $N$ are marked in bold). In this SNR range of $13 \mathrm{~dB}$, the noise impact is significantly low, allowing the QSR strategy to work. On the other hand, the constellation keeps a good condensation as well, so the CSR strategy provides a high reduction. As already mentioned in previous sections, both strategies can be combined by using the QCSR algorithm. For the symbols obtained with QSR, only those distances that are not repeated are computed, and the rest are replicated, as CSR does. In this case, the final reduction comes to $N_{Q C S R}=66$ distances.

Fig. 4.6 shows the performance comparison with the final selected parameters $N$, using 4 representative CRs: a low CR 2/15, a medium CR 6/15 (recently analyzed) and two high CRs $10 / 15$ and $13 / 15$. The idea is to show separately the contribution of each strategy to the proposed algorithm, observing the impact on the system performance. 


\section{CHAPTER 4. LOW-COMPLEXITY DEMAPPING AND QUANTIZATION ALGORITHMS}
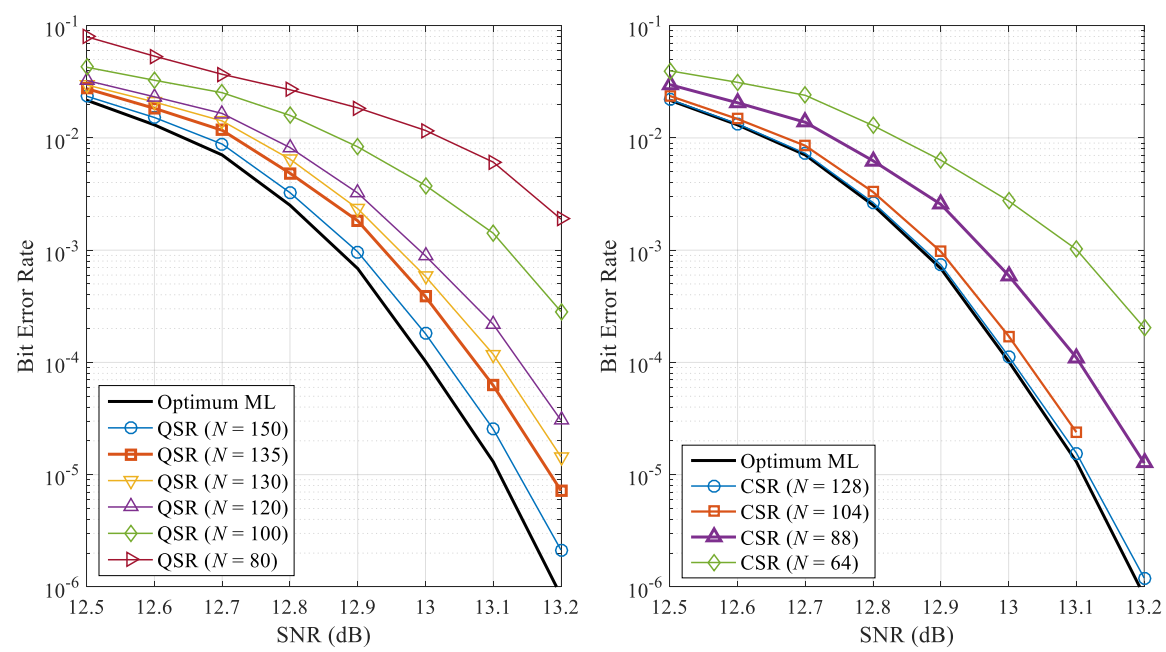

Figure 4.5: Performance of QSR (left) and CSR (right) demappers, depending on the number of distances $N$ and compared with the optimum ML demapper. Results presented for a CR 6/15, under i.i.d. Rayleigh channel.

With low CRs such as 2/15 the SNR required is very low, and then the constellations are compressed. The compression allows the CSR strategy to work especially well, obtaining a reduction in the number of distances from up to $N_{C S R}=16$, with no performance loss. However, precisely the low SNR hampers the quadrant search. The QSR strategy often is erroneous, and needs to calculate almost all distances, having $N_{Q S R}=235$. Therefore, in this particular case the QCSR algorithm works also with only $N_{Q C S R}=16$. Although the quadrant search fails, the algorithm only needs to consider the 16 unique distances that are not repeated, replicating the rest and providing the correct information to the LLR computation. With the high CR 10/15, the CSR strategy hardly works, as the constellations are barely condensed. With CSR, it is necessary to use $N_{C S R}=224$ distances. Nevertheless, the low noise impact allows QSR to work better, needing just $N_{Q S R}=90$. Combining both techniques, the final reduction is $N_{Q C S R}=77$. On the other hand, the constellation optimized for the CR $13 / 15$ is not condensed at all, considering that each constellation symbol is unique in the I/Q plane. Hence, the QCSR algorithm is based only in the QSR strategy for this case, calculating $N_{Q C S R}=80$ distances.

Fig. 4.7 summarizes the minimum number of distances $N$ for the proposed algorithm, for all CRs from 2/15 to 13/15. With CSR, the higher contribution 


\subsection{Performance Evaluation: Minimum Number of Distances}
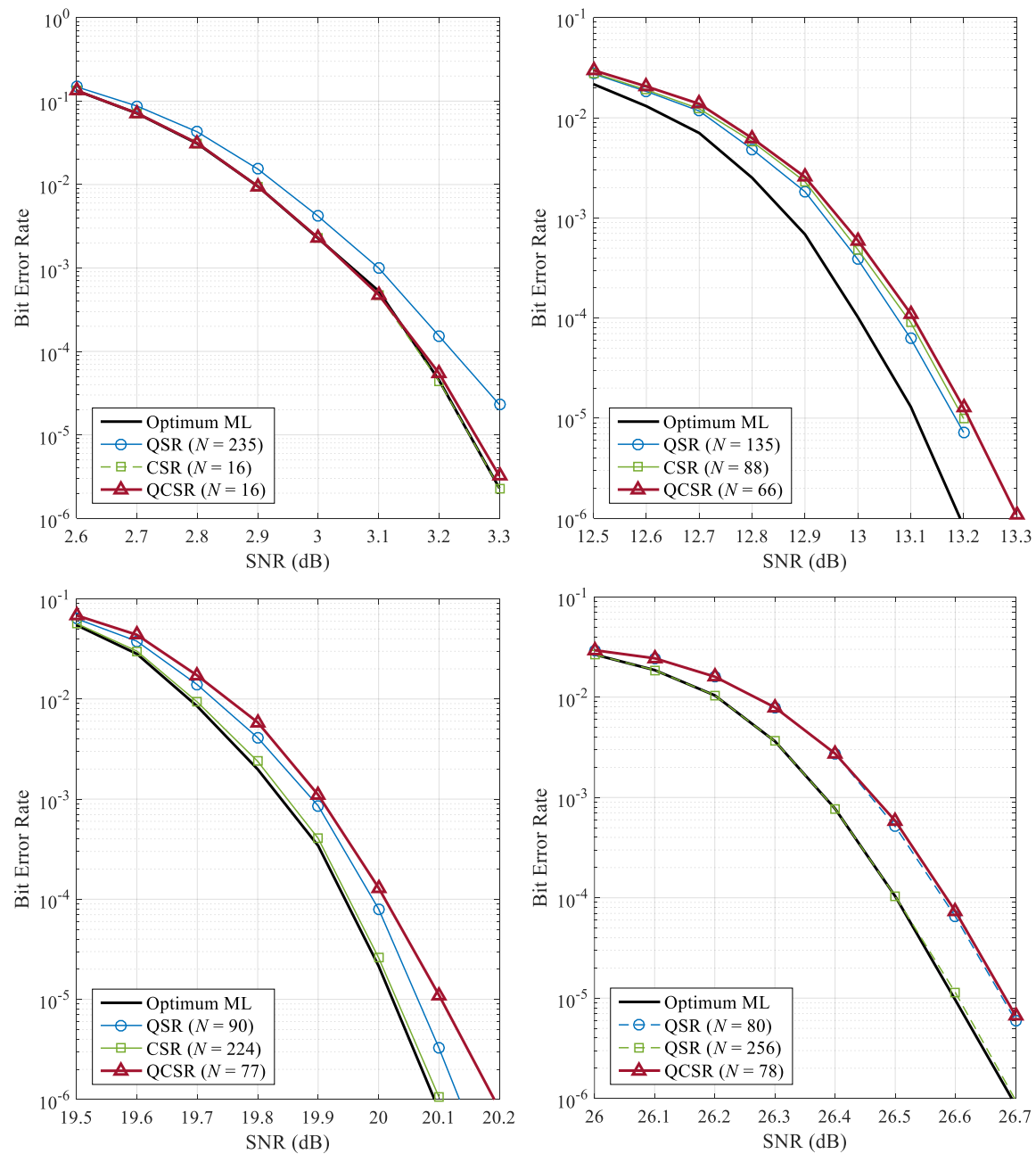

Figure 4.6: Performance comparison of ML and QCSR demappers. QSR and CSR strategies are also shown by separate. Results presented for code rates $2 / 15$ (top left), 6/15 (top right), 10/15 (bottom left) and 13/15 (bottom right), under i.i.d. Rayleigh channel.

is achieved for low CRs, where the number of operations can be reduced up to $N_{C S R}=16$, which represents a reduction of $93 \%$. Regarding the contribution of QSR, it can be seen that the curve is directly related with the probability of selecting an erroneous quadrant $\left(p_{e}\right)$, shown in Fig. 4.3. However, the performance of QSR becomes better for high CRs, especially from 8/15 to 


\section{CHAPTER 4. LOW-COMPLEXITY DEMAPPING AND QUANTIZATION ALGORITHMS}

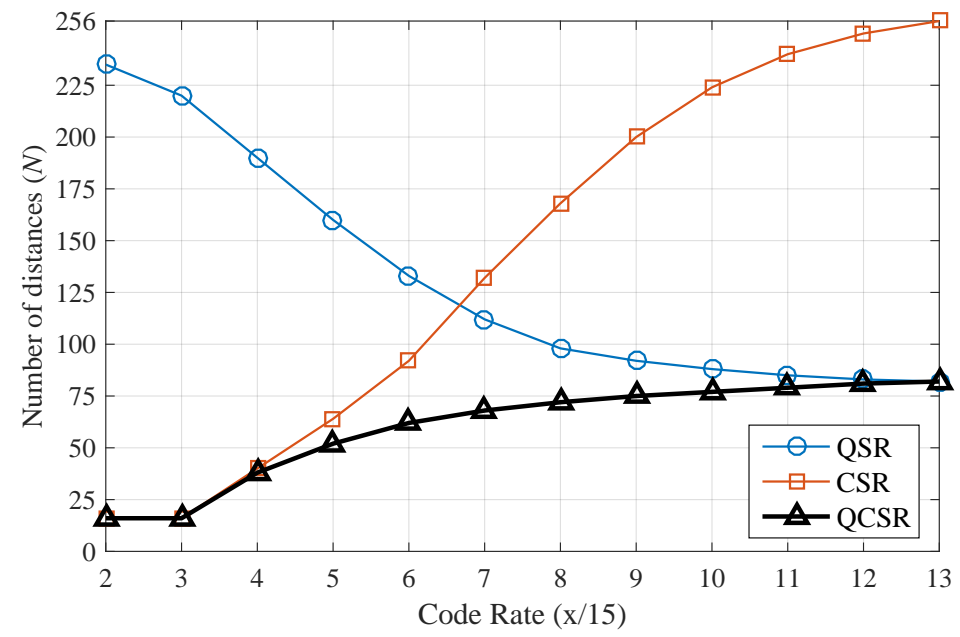

Figure 4.7: Minimum number of distances $N$ required for all proposed algorithms. 2D256NUC and CRs from 2/15 to 13/15, under i.i.d. Rayleigh channel.

13/15. When combining the two strategies into one single algorithm, it becomes especially effective for medium CRs, where both advantages can be taken into account. Fig. 4.8 shows the final reduced constellations used with QCSR when the first quadrant is transmitted, for the representative CRs analyzed: $2 / 15,6 / 15,10 / 15$ and $13 / 15$. In a real receiver, the complexity reduction is determined by the maximum number of distances $N$ to be computed. With the combined QCSR demapper, the maximum value was obtained for the CR $13 / 15$, with $N_{Q C S R}=80$, which implies a reduction in the number of required operations of $69 \%$.

\subsubsection{Performance Loss with Alternative Channel Models}

This section provides the performance loss of QCSR for different channel models, for which the algorithm has not been optimized, under ideal and realistic channel estimation conditions. The idea is to keep the same number of distances, $N_{Q C S R}$, computed for i.i.d. Rayleigh channel and ideal channel estimation (see Fig. 4.7), and observe the additional loss introduced. Table 4.2 shows the performance loss obtained with white Gaussian noise (AWGN), $F_{1}$ Riceanfading and $P_{1}$ Rayleigh-fading channel models, defined in [28], compared to the results obtained in the analysis for i.i.d. Rayleigh channel model, under ideal channel estimation conditions. Note that the $F_{1}$ channel is used to describe 


\subsection{Performance Evaluation: Minimum Number of Distances}
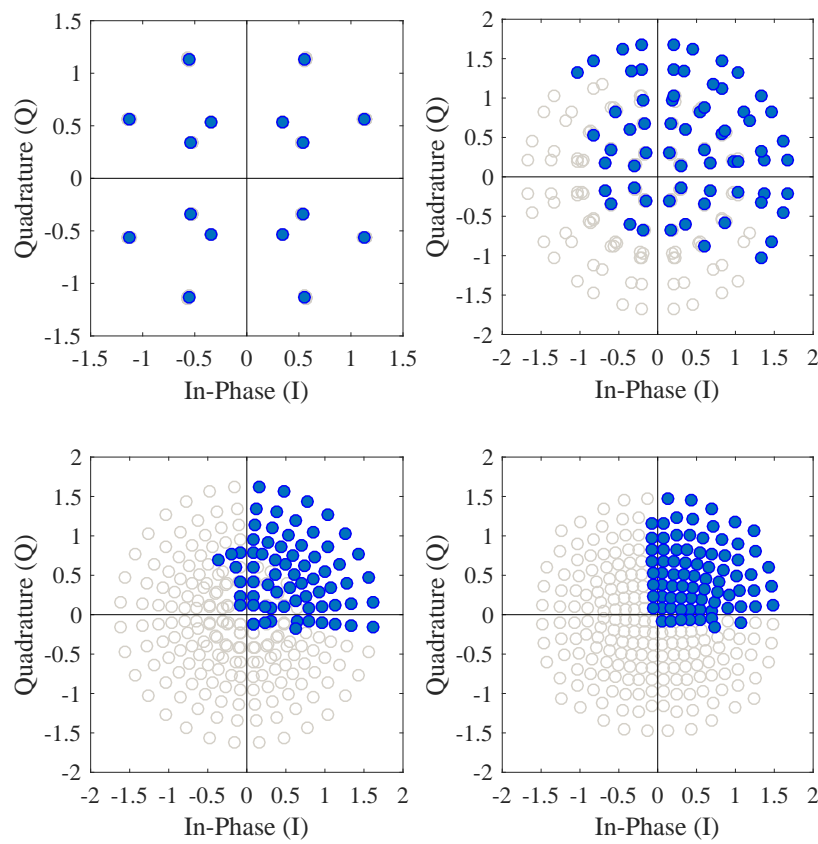

Figure 4.8: Result of reduced 2D-256NUCs with QCSR, for CRs 2/15 (top left), 6/15 (top right), 10/15 (bottom left) and 13/15 (bottom right), i.i.d. Rayleigh channel.

the fixed outdoor rooftop-antenna reception conditions, and the $P_{1}$ channel is used to describe the portable indoor or outdoor reception conditions.

With AWGN, the performance loss remains under $0.1 \mathrm{~dB}$ for all considered CRs. The SNR required is lower than the obtained for i.i.d. Rayleigh channel, allowing the QCSR algorithm to work better. The $F_{1}$ channel model with fixed reception, and therefore with a direct path (line-of-sight ray) also permits lower SNRs than the i.i.d. Rayleigh channel. For this reason, the performance loss of QCSR also remains under $0.1 \mathrm{~dB}$. However, the same does not occur with the $P_{1}$ portable channel. Having a less selective channel implies higher SNRs. Higher noise levels entail a worse result of the QCSR algorithm. In this case, the performance loss is up to $0.2 \mathrm{~dB}$ with high CRs.

Table 4.3 shows the performance loss of QCSR obtained under realistic channel estimation conditions. With real channel estimation, the channel fading $h$ received is different from the transmitted one. In order to obtain $h$, a linear frequency and time interpolation from the Pilot Pattern (PP) is done. 


\section{CHAPTER 4. LOW-COMPLEXITY DEMAPPING AND QUANTIZATION ALGORITHMS}

\begin{tabular}{|c|c|c|c|c|c|}
\cline { 3 - 6 } \multicolumn{2}{c|}{} & \multicolumn{5}{c|}{ Code Rate } \\
\hline Channel & Parameter (dB) & $\mathbf{2 / 1 5}$ & $\mathbf{6 / 1 5}$ & $\mathbf{1 0} / \mathbf{1 5}$ & $\mathbf{1 3 / 1 5}$ \\
\hline \multirow{2}{*}{ i.i.d. Rayleigh } & ML: SNR & 3.2 & 13 & 19.9 & 26.5 \\
\cline { 2 - 6 } & QCSR: Loss & $<0.1$ & $<0.1$ & $<0.1$ & $<0.1$ \\
\hline \multirow{2}{*}{ AWGN } & ML: SNR & 1.8 & 10.6 & 17.1 & 22.2 \\
\cline { 2 - 6 } & QCSR: Loss & $<0.1$ & $<0.1$ & $<0.1$ & $<0.1$ \\
\hline \multirow{2}{*}{$F_{1}$} & ML: SNR & 2.7 & 11.3 & 18 & 23.2 \\
\cline { 2 - 6 } & QCSR: Loss & $<0.1$ & $<0.1$ & $<0.1$ & $<0.1$ \\
\hline \multirow{2}{*}{$P_{1}$} & ML: SNR & 4.6 & 13.6 & 21.3 & 28.7 \\
\cline { 2 - 6 } & QCSR: Loss & $<0.1$ & 0.2 & 0.2 & 0.2 \\
\hline
\end{tabular}

Table 4.2: QCSR performance loss under ideal channel estimation conditions.

\begin{tabular}{|c|c|c|c|c|c|}
\cline { 3 - 6 } \multicolumn{2}{c|}{} & \multicolumn{4}{c|}{ Code Rate } \\
\hline Channel & Parameter (dB) & $\mathbf{2 / 1 5}$ & $\mathbf{6 / 1 5}$ & $\mathbf{1 0 / 1 5}$ & $\mathbf{1 3 / 1 5}$ \\
\hline \multirow{2}{*}{$F_{1}$} & ML: SNR & 3.7 & 12.2 & 18.8 & 24 \\
\cline { 2 - 6 } & QCSR: Loss & $<0.1$ & $<0.1$ & $<0.1$ & $<0.1$ \\
\hline \multirow{2}{*}{$P_{1}$} & ML: SNR & 5.6 & 14.2 & 22 & 29.1 \\
\cline { 2 - 6 } & QCSR: Loss & $<0.1$ & 0.2 & 0.2 & 0.3 \\
\hline
\end{tabular}

Table 4.3: QCSR performance loss under realistic channel estimation conditions.

In the case of study, two different pilot patterns, i.e. SP24_4 and SP12_2 from ATSC 3.0 [15], are used for the $F_{1}$ and $P_{1}$ channel models respectively [28].

The higher performance loss of QCSR is obtained for the $P_{1}$ channel model, with $0.3 \mathrm{~dB}$ of difference in relation to the optimum ML demapper for the CR 13/15. These results confirm the robustness of the QCSR algorithm. QCSR requires, for all studied cases, SNRs nearby to the optimum ML demapper, feasible for its implementation in real receivers where the obtained performance loss is almost negligible.

\subsubsection{QCSR with Non-Uniform Rotated Constellations}

The QCSR algorithm can be also applied to the non-uniform rotated constellations optimized in Chapter 3. Note that there is no complexity increase when rotating 2D-NUCs. The QCSR demapper can be used in order to reduce the complexity in a similar way than in previous sections. The rotation does not influence the condensation, so this part of the algorithm remains identical. Only a slight change in the first stage of QSR is required, since it is necessary to reorganize the new $N$ rotated symbols by probability order, i.e., to obtain new histograms. 


\subsection{Digital Quantization of LLR and I/Q Components}
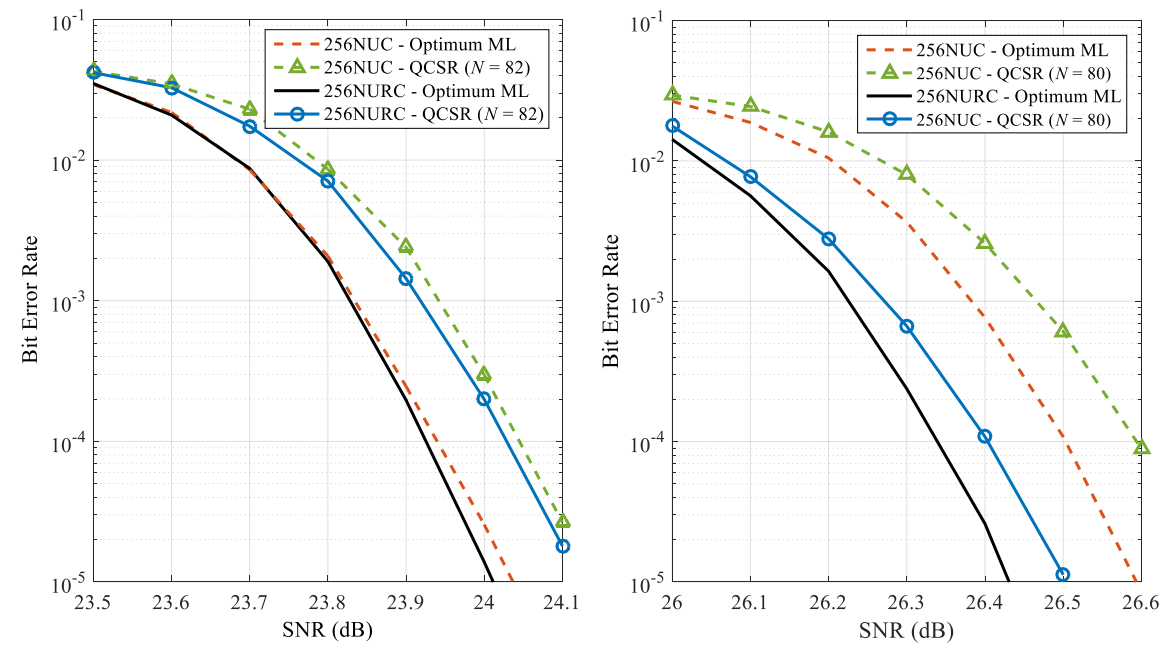

Figure 4.9: Performance comparison of ML and QCSR demappers, with and without rotation. Results presented for 256QAM and code rates 12/15 (left) and 13/15 (right), i.i.d. Rayleigh channel.

Fig. 4.9 shows the BER performance of the QCSR demapper with and without rotation, compared with the optimum ML demapper, for CRs 12/15 and $13 / 15$. Note that only these CRs are examined, since no rotation gain is obtained for lower CRs and 256NUCs. For CR 12/15, the SNR performance of QCSR is very similar with and without rotation. However, the same does not occur with 13/15, where the SNR gain of the QCSR rotated demapper is also $0.2 \mathrm{~dB}$ (as the rotated ML), and the number of distances is maintained, with $N=80$. Furthermore, compared to the non-rotated ML demapper, the QCSR rotated demapper has a $0.1 \mathrm{~dB}$ gain, and a complexity reduction of $69 \%$.

\subsection{Digital Quantization of LLR and I/Q Com- ponents}

Quantization [67] can be defined as the procedure of constraining a continuous set of values to a discrete digital signal that can be processed, transmitted or stored in real digital processors. As reference [27] mentions, signal accuracy increases with the number of quantization bits, but high resolution signals are more costly to store and process. A low quantization resolution is hence desired for hardware implementations, since it implies a reduction of chip area, and 


\section{CHAPTER 4. LOW-COMPLEXITY DEMAPPING AND QUANTIZATION ALGORITHMS}

reduced manufacture cost and power consumption. In broadcast receivers, the highest memory requirements are always needed in the time interleaving block, since systems face signal strength fluctuations over the time, which are even higher in case of mobile reception. The memory required to store a transmit block depends on the number of quantization levels and their distribution [67]. More levels imply greater fidelity but also need more memory to store. Thus, there is a clear trade-off between in-chip memory and receiver performance.

Conventional receivers usually perform TDIL immediately before the demapping process takes place, but both operations can be swapped, minimizing memory requirements without any additional performance loss. In other words, the TDIL can be performed after the demapper, so instead of quantizing I and Q symbols, LLRs are used. Naturally, the use of NUCs affects the quantization process, since both I/Q components or LLR values after the demapper are completely different from those obtained with QAM. In this section, optimum quantizers in the sense of maximum BICM capacity are investigated. These quantizers are evaluated depending on system parameters such as receiver resolution, code rate and constellation order.

\subsubsection{System Model and Considered Scenario}

Following the approach given in [27], two possible receiver schemes that differ in their TDIL structure are investigated in this thesis. For the first scheme, time de-interleaving is performed directly on the I and Q components of the received signal. In this case, the components of received symbols and estimated channel gains are quantized prior to TDIL. The second scheme realizes the quantization in a different point of the receiver chain. With this architecture, demapping (the process of obtaining soft information bits from the received symbol) is performed prior to time de-interleaving. Thus, quantization is performed on the demapped LLRs, which are subsequently stored in the TDIL.

\section{Receiver Implementation}

Analog-to-digital converters in real receivers perform two fundamental operations, signal sampling and quantization. First, the continuous input signal is converted into digital with a particular sampling rate. It must fulfil the Nyquist rate-signaling criterion, with a sampling rate higher than twice the highest frequency of the input signal [85]. Afterwards, the quantizer maps the obtained sampled values to a discrete set of $2^{q}$ values, where $q$ is the number of quantization bits or resolution [106]. The goal of the quantizer is to represent the input signal with a very small distortion, using the lowest number of $q$ bits possible. Note that the final amount of memory in bits required to store the samples of 


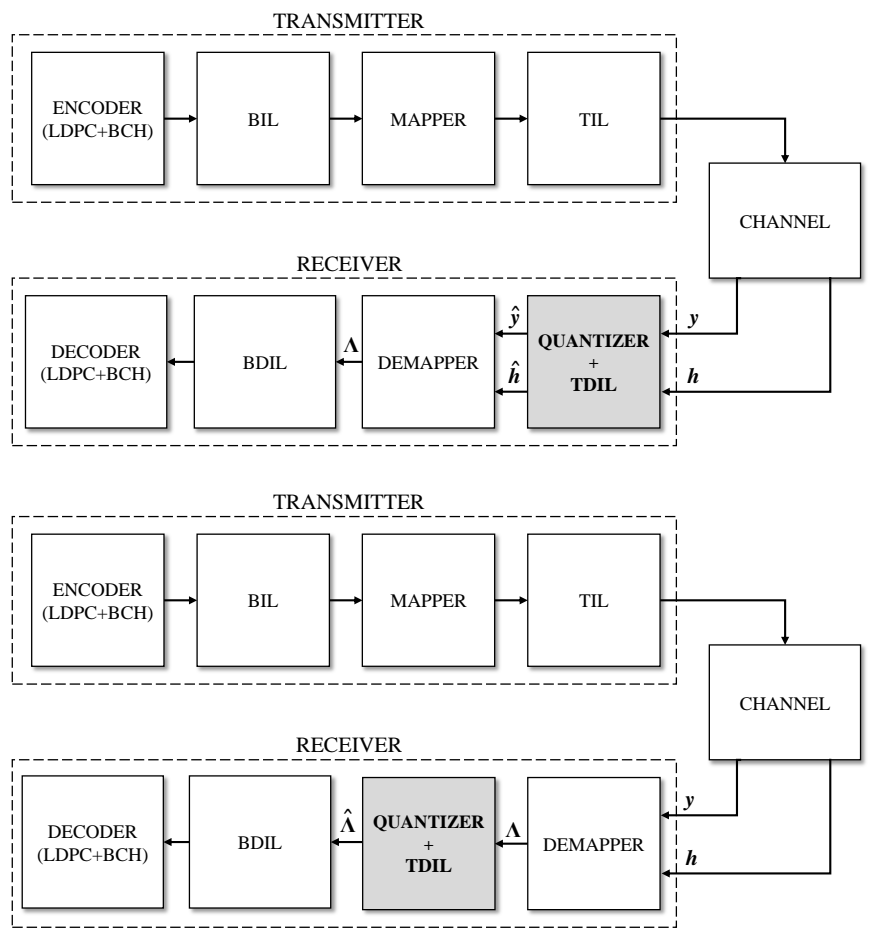

Figure 4.10: Basic diagram schemes of I/Q signal quantization (top) and LLR quantization (bottom).

the previous processing blocks directly depends on the resolution of the quantization algorithms. Fig. 4.10 shows the two different schemes considered in this thesis. At the transmitter, the constellation symbols are time-interleaved and transmitted through the channel. However, at the receiver, both schemes implement signal quantization to store received information at the TDIL memory, but at different parts of the receiving chain.

The first scheme (Fig. 4.10 top) realizes the quantization before the demapper, where received constellation symbols $y$ and the channel estimation gains $h$ are stored in order to perform time de-interleaving. Before storing into the time de-interleaving memory, the I and Q components of both signals are quantized. In this approach, the quantized symbols are stored prior to time de-interleaving, until the TDIL memory is filled. Afterwards, the demapper receives the quantized symbols $\hat{y}$ and quantized channel estimation gains $\hat{h}$ to calculate the corresponding LLRs. The number of LLRs calculated depend on the constella- 


\section{CHAPTER 4. LOW-COMPLEXITY DEMAPPING AND QUANTIZATION ALGORITHMS}

tion order used. The computed LLRs finally pass through the BDIL and LDPC decoder blocks, and resulting bits are used for error measurements.

The second scheme (Fig. 4.10 bottom) swaps the TDIL and demapping blocks, and therefore performs quantization in a different manner. In this case, the demapper is calculates the LLRs directly from the received constellation symbols $y$ and channel estimates $h$, before the TDIL. In [69], it is shown that if the TDIL block de-interleaves groups of LLR corresponding to the received symbols and channel estimates, there is no effect in the performance. The output LLRs $(\Lambda)$ at the demapper are then quantized, obtaining $\hat{\Lambda}$. Once the TDIL is filled, the groups of quantized LLRs are time de-interleaved. Finally, output LLRs pass through the BDIL and are fed to the LDPC decoder.

\section{Uniform and Non-Uniform Quantization}

A scalar quantizer can be defined as a set of disjoint intervals, $S=\left\{S_{i} ; i \epsilon I\right\}$, where the index set $I$ is a collection of consecutive integers beginning with 0 or 1 , together with a set of reproduction values $R=\left\{z_{i} ; i \epsilon I\right\}$. The overall quantizer is defined by $Q(x)=z_{i}$, for $x \in S_{i}$, and can be expressed as:

$$
Q(x)=\sum_{i} z_{i} s_{i}(x)
$$

where the indicator function $s(x)$ is 1 if $x \epsilon S$ and 0 otherwise. We also define $S_{i}=\left[a_{i}, a_{i+1}[\right.$ as the boundaries to define each region $i$. The set of reproducers presented in Eq. 4.5 can be equally or non-equally spaced, considering uniform or non-uniform quantization. Note that this concept is totally different from uniform and non-uniform constellations, where the distribution refers to constellation symbols. With uniform quantization, real and imaginary parts are quantized separately and uniformly. A quantizer is said uniform when the levels $z_{i}$ are equispaced. With non-uniform quantization, reproducers are no longer equispaced. An example of the possible uniform and non-uniform reproducers is shown in Chapter 2, Fig. 2.12.

\section{Considered Scenario}

Reproducer values are optimized for a DVB-NGH mobile channel model with user speed $60 \mathrm{~km} / \mathrm{h}$, since communication in motion experiences a certain Doppler shift, and TIL is performed in order to mitigate it. It is also better to transmit very robust transmission modes and hence, low-order constellations are examined, i.e. 16NUC, 64NUC and 256NUC with CRs from ATSC 3.0, from $2 / 15$ to $13 / 15$ with step $1 / 15$. The simulations include inner LDPC codes with a word length size of 64800 bits. The simulated system uses 8192 sub- 
carriers and a cyclic prefix of $1 / 4$ to trade-off cell area and resilience against a $33 \mathrm{~Hz}$ Doppler spread in a $6 \mathrm{MHz}$ bandwidth. For the performance evaluation, we transmit and decode a TIL block with $16 \mathrm{~m}$ LDPC codewords, being $m=\log _{2}(M)$ and $M$ the number of constellation symbols. It was confirmed with simulations that using more LDPC codewords within the TIL block does not have a significant impact on the performance. With QAM constellations, reproducers for I/Q symbols or LLRs are obtained at different SNR targets. With NUCs, the procedure is slightly different. Since each NUC is designed for a specific CR, it is necessary to calculate the LLR and I/Q reproducer values for each constellation and CR combination, already optimized for a particular SNR. The SNR in the optimization is selected as the value that provides a bit error rate $(\mathrm{BER})$ of $10^{-4}$, when using QAM constellations.

\subsubsection{Quantization of Log-Likelihood Ratios}

The proposed algorithm maximizes the BICM capacity of the system under study, which can be defined as the highest rate achievable (in the sense of asymptotically vanishing error probability) in a BICM system with independent parallel channels. It takes LLR quantized reproducers as initial inputs, and iteratively seeks the optimum capacity by changing those reproducers, making use of the Nelder-Mead algorithm [89]. The BICM capacity is calculated as the combination of capacities of all bits modulated onto a constellation symbol, using an optimum ML demapper.

From the received symbol vector $y$, and the channel vector $h$, each LLR $\Lambda_{l}$ is computed and quantized for all code bits $c_{l}, l=1, \ldots, m$, with $m$ as the number of bits per constellation symbol. The expression of a single computed and quantized $\hat{\Lambda}_{l}$ can be denoted as follows:

$$
\hat{\Lambda}_{l}=Q\left[\Lambda_{l}\right] \triangleq Q\left[\log \frac{\sum_{x \in \chi_{l}^{1}} p(y \mid x, h)}{\sum_{x \in \chi_{l}^{0}} p(y \mid x, h)}\right]
$$

where $Q$ denotes the quantization operation. $\chi_{l}^{1}$ and $\chi_{l}^{0}$ denote the complementary sets of transmit vectors for which $c_{l}=1$ and $c_{l}=0$ respectively. Naturally, the BICM calculation is affected since it depends on the densities of quantized symbols and LLRs $p(y \mid x, h)$. Rather than using a single quantizer for all LLRs, the proposed method looks for the optimum reproducer values for each bit position, modulation and CR. Depending on the constellation order, it is necessary to quantize up to $m$ different statistics, one per each LLR. With QAM and 1D-NUC, the number can be reduced to $m / 2$, since I and Q compo- 


\section{CHAPTER 4. LOW-COMPLEXITY DEMAPPING AND QUANTIZATION ALGORITHMS}
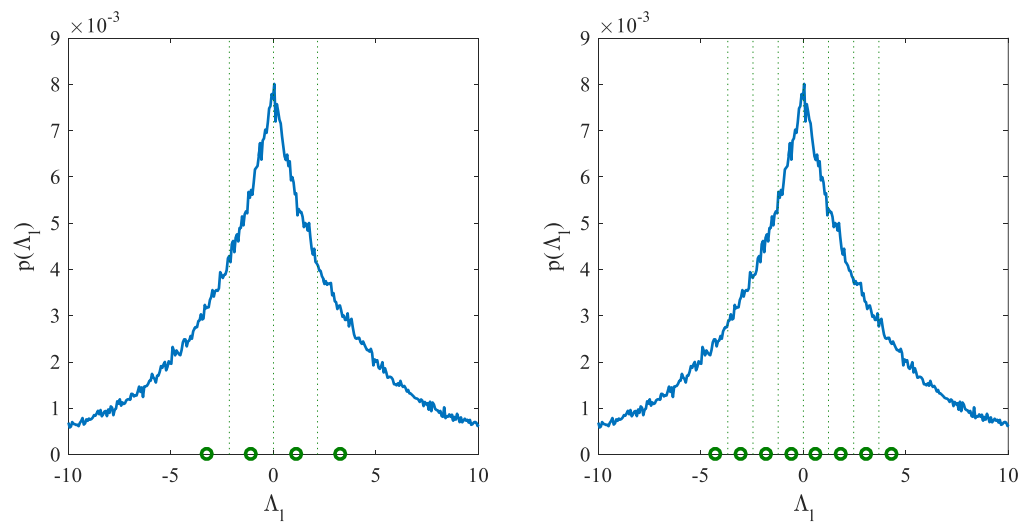

Figure 4.11: Example of probability density function and uniform quantized values of $\Lambda_{1}$, for a received SNR of $10 \mathrm{~dB}, 64 \mathrm{NUC}$. Reproducers and boundaries are shown for 2 (left) and 3 (left) bits.

nents are repeated, and therefore odd LLRs can be replicated from even ones. However, with 2D-NUCs all LLR statistics need to be quantized separately.

\section{Uniform Quantization}

With uniform quantization, LLRs are quantized using uniform spacing of the reproducers and boundaries. Since reproducers are uniformly spaced, it is only necessary to obtain the maximum LLR quantized value of all reproducers, which simplifies drastically the optimization process. The rest of reproducers can be calculated from it.

Although the quantization does not require information about $p\left(\Lambda_{l}\right)$, it helps to understand the optimization and final values of quantization reproducers. Fig. 4.11 shows an example of the probability density function and uniform quantized values of $\Lambda_{1}$ (most significant bit) for 64 NUC designed for a SNR of $10 \mathrm{~dB}$, directly related to a CR 5/15. As can be observed, the higher the number of bits, the larger the maximum reproducer value. With a higher number of memory bits available, more reproducers can be used to quantize, obtaining a larger maximum value that covers better the LLR range. In this case, maximum values of approximately 3.23 and 4.30 are obtained, using 2 and 3 bits to quantize respectively. With $64 \mathrm{NUC}$, the procedure has to be extended to $m=6$ different quantizers, one per each $\Lambda_{l}$. For later performance results, it is only necessary to store in a look-up table (LUT) the maximum values, and the rest can be simply calculated depending on the number of bits. As an 


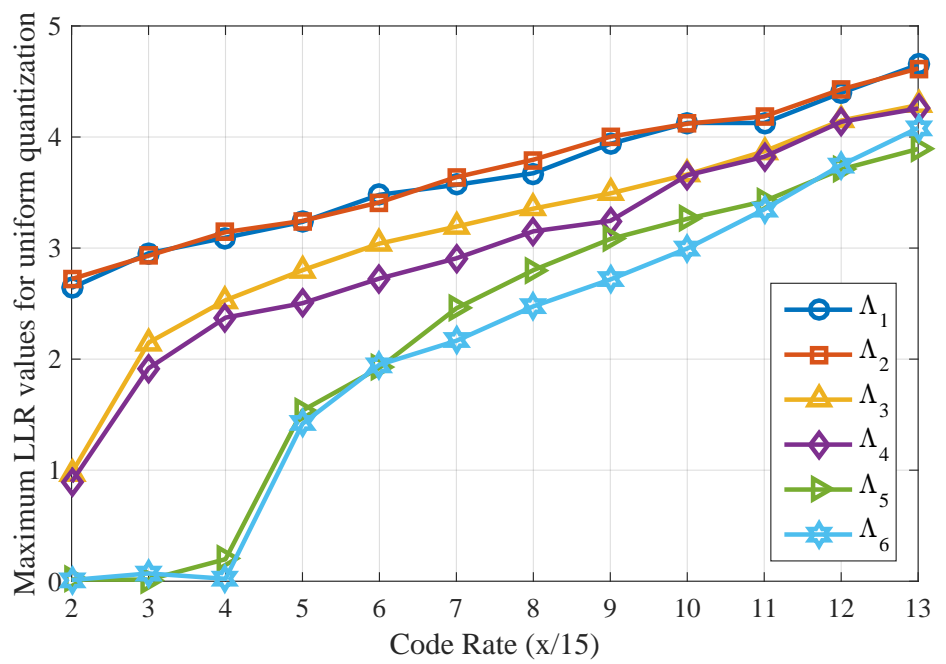

Figure 4.12: Maximum LLR reproducers, for all $\Lambda_{l}$. Results are shown for 64 NUC, optimized for 2 bits.

example, Fig. 4.12 depicts the $\Lambda_{l}$ maximum values for a 64 NUC optimized for all CRs considered, using 2 bits per LLR.

The lower the CR used, and therefore the SNR for which the constellation is optimized, the smaller the maximum LLR reproducer. This comes from the condensation that NUCs suffer. At low SNRs, the LLR values coming from the demapper are extremely low, due to the very high noise impact. The information of the two least significant bits is forced to zero, so reproducers are close to that value. This is unique from NUCs, since QAM does not show any threshold effect for this specific bits. The very low soft-decision values indicate the LDPC a very weak reception. This clearly has an impact in the number of bits required. On the other hand, the quantized values for the most significant LLRs are high, due to the larger distance between symbols with the same bit in the I/Q space.

\section{Non-Uniform Quantization}

With non-uniform quantization, the optimum reproducers are also obtained through an algorithm that maximizes the BICM capacity. Unlike uniform quantization, all positive reproducer values need to be optimized separately. Due to the left-right and up-down symmetry of NUCs, negative reproducers can be derived from positive ones. Therefore, the number of reproducers to 


\section{CHAPTER 4. LOW-COMPLEXITY DEMAPPING AND QUANTIZATION ALGORITHMS}

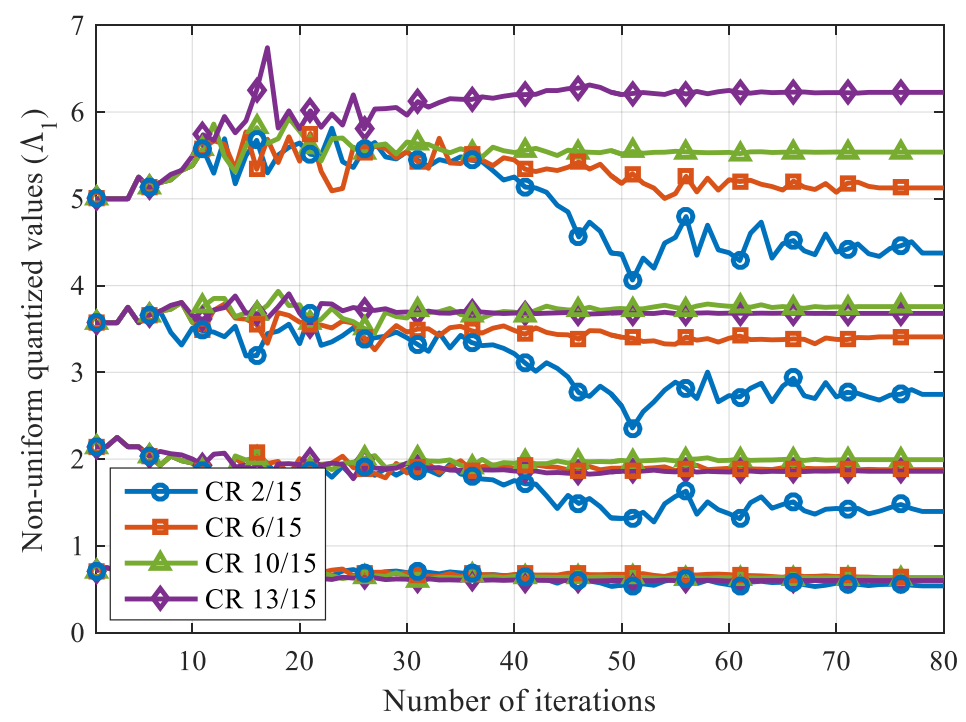

Figure 4.13: $\Lambda_{1}$ quantized values with recursive non-uniform optimization, for different $64 \mathrm{NUCs}$ optimized for CRs $2 / 15,6 / 15,10 / 15$ and 13/15. 3 quantization bits, i.e. 4 positive levels.

optimize is $2^{q-1}$, being $q$ the number of quantization bits selected. As expected, more degrees of freedom are directly related with a better performance, since the capacity can be further optimized, which can be translated into less TDIL memory required at the receiver.

Fig. 4.13 depicts an example of a non-uniform optimization process, using the algorithm defined in [89]. Reproducer values are shown for different iterations and a quantized $\Lambda_{1}$, using 3 bits, which means that 4 positive reproducers are optimized. The optimization is shown for 4 different 64 NUCs, designed for CRs $2 / 15,6 / 15,10 / 15$ and 13/15. Initial values are taken from uniform quantization. The optimized reproducers change differently depending on the shape of the constellation and SNR. Optimized reproducers are uniform-like when using low CRs. A different situation is obtained with high CRs, where strongly non-equispaced reproducer values are obtained.

\subsubsection{Quantization of I/Q Components}

For the scheme considered in Fig. 4.10 (top), the signals $y$ and $h$ need to be quantized. As with LLRs, $2^{q}$-level scalar quantizers are considered. In this case, quantizers do not depend on the code bit position. I and $\mathrm{Q}$ components 


\subsection{Digital Quantization of LLR and I/Q Components}

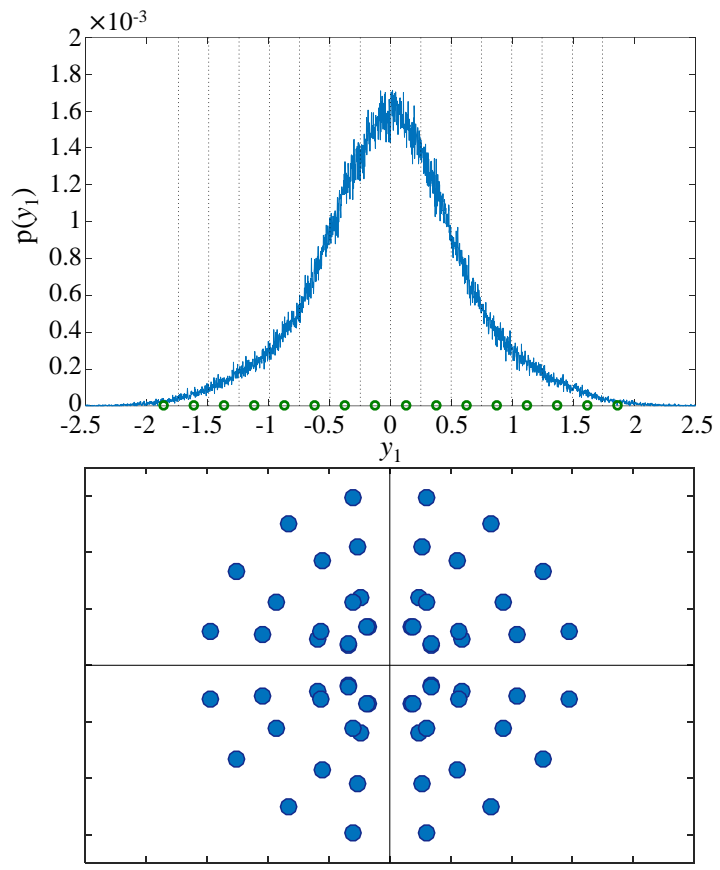

Figure 4.14: Probability density function and uniform quantized values of the I component with 4 bits, for a received SNR of $11 \mathrm{~dB}$ (CR 6/15). The corresponding 64NUC used is also shown.

of received symbols $y$ and channel estimates $h$ are quantized independently. In this case:

$$
\Lambda_{l} \triangleq \log \frac{\sum_{x \in \chi_{l}^{1}} p(\hat{y} \mid x, \hat{h})}{\sum_{x \in \chi_{l}^{0}} p(\hat{y} \mid x, \hat{h})}
$$

where $\hat{y}=Q_{1}[y]$ and $\hat{h}=Q_{2}[h]$ are the quantized receive signal and channel coefficient, respectively, and $p(\cdot)$ refers to the conditional probability density function of $\hat{y}$ given $x, \hat{h}$. Naturally, the BICM calculation is affected since it depends on the densities of quantized symbols and LLRs. It should be noted that received symbols and channel estimates are quantized in a different manner. While receive signal components need different reproducers for each NUC and CR combination, channel estimates require only one quantizer design, since they are independent from the SNR. 


\section{CHAPTER 4. LOW-COMPLEXITY DEMAPPING AND QUANTIZATION ALGORITHMS}

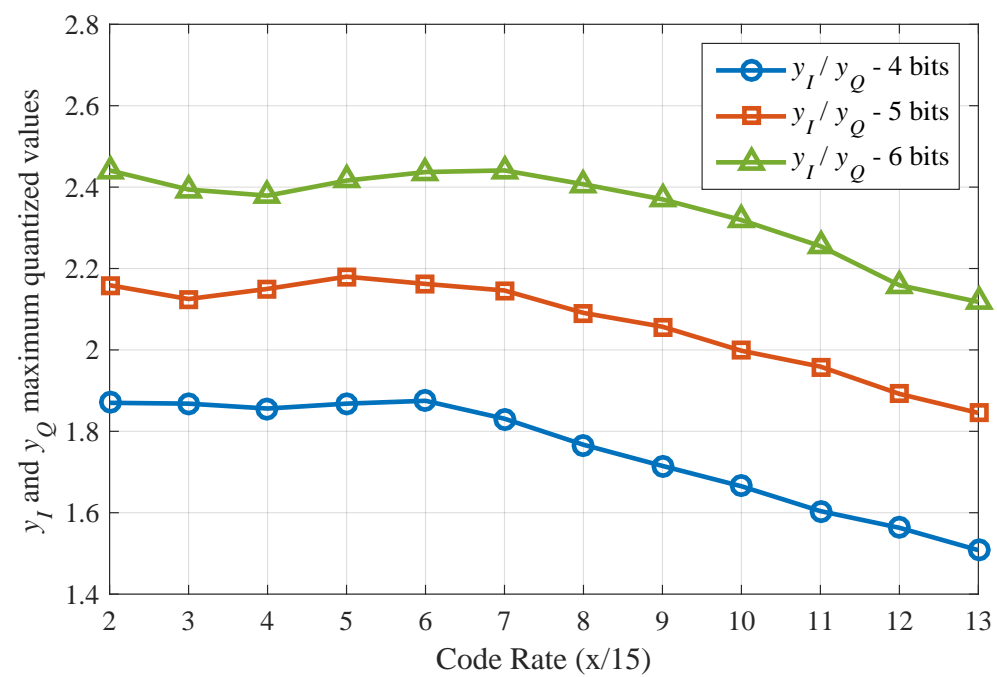

Figure 4.15: Maximum quantized values of I and $\mathrm{Q}$ components $\left(y_{I}\right.$ and $\left.y_{Q}\right)$. Results are shown for $64 \mathrm{NUC}$, quantized using 4,5 and 6 bits.

\section{Uniform Quantization}

In this section, $y$ and $h$ are quantized using a uniform spacing of reproducers and boundaries. As with LLRs, it is only necessary to obtain the maximum quantized reproducer. The rest of reproducers can be calculated from it. Fig. 4.14 shows an example of the probability density function and uniform reproducers with 4 bits of the I component of $y$. The corresponding $64 \mathrm{NUC}$ used is also shown in Fig. 4.14. The constellation was designed for a SNR of $11 \mathrm{~dB}$, which is related to CR 6/15. Similar values are obtained for the Q component. When I and Q components are quantized, more bits are required compared to LLRs, in order to obtain similar BICM capacities. However, only 4 components need to be quantized, which is a potential advantage when using high-orders of constellation. Maximum reproducer values of $1.86,2.17$ and 2.43 are obtained for the I component, using 4, 5 and 6 bits respectively.

The quantizer parameters for the receive signal have to be designed and stored for all CR combinations. In contrast, the quantizers for the channel coefficients need to be designed and stored only once. Fig. 4.15 shows the maximum reproducers of $\mathrm{I}$ and $\mathrm{Q}$ components for the received signal $y$, with a 64NUC and the considered channel model. I and Q quantizers are identical, due to symmetry. With NUCs, large maximum quantized values are required for low CRs. At low SNRs, the very high noise impact affects the received 
symbol, requiring higher reproducers. However, slightly lower values are needed with NUCs, due to the condensation. Regarding the channel estimates, the maximum reproducer values are $1.33,1.54$ and 1.73 for both components, using 4,5 and 6 bits respectively. As mentioned above, these reproducers do not depend on the constellation shape and SNR. Note that these values may vary, if different channel conditions are selected.

\section{Non-Uniform Quantization}

With non-uniform quantization, all positive reproducers are optimized separately. Due to the symmetry of quantized symbols, all negative reproducers can be derived from positive ones. Therefore, the degrees of freedom are again $2^{q-1}$. Compared to uniform quantization, the most significant improvement in terms of BICM capacity is achieved using high CRs. In next sections, we also analyze the performance of non-uniform quantization of I and Q components.

\subsection{Quantization Loss vs. Time De-Interleaving Memory Trade-Off}

In this section, ATSC 3.0 physical layer simulation results are provided to illustrate the performance achieved with LLR and I/Q quantization for different constellations and CR combinations, and different quantizer resolutions. ML demapping is used. To provide reliable results, the performance is evaluated under different channel realizations for each SNR, i.e., different seeds for the (pseudo) random generators for each SNR. For a given SNR, all receiver configurations are simulated under the same set of channel realizations. For each SNR a maximum number of $10^{4}$ and a minimum number of $10^{3}$ TIL blocks are considered. The simulation is stopped when 100 erroneous TIL blocks are detected. A TIL block is considered erroneous when an erroneous bit is detected in the entire TIL block. Finally, the selected quality of service for comparisons is a bit error rate (BER) of $10^{-4}$ after $\mathrm{BCH}$ (Bose-Chaudhuri-Hochquenghem) decoding.

\subsubsection{Performance Loss of LLR Quantization}

This subsection evaluates the performance achieved with LLR quantization in the considered scenario, using the reproducers saved in the LUT. For the sake of simplicity, we show results using $64 \mathrm{NUCs}$, but the complete process is extended to 16NUCs and 256NUCs. In Fig. 4.16, the particular case of CR $13 / 15$ is shown, since it is the least robust mode, and more bits are required to 


\section{CHAPTER 4. LOW-COMPLEXITY DEMAPPING AND QUANTIZATION ALGORITHMS}

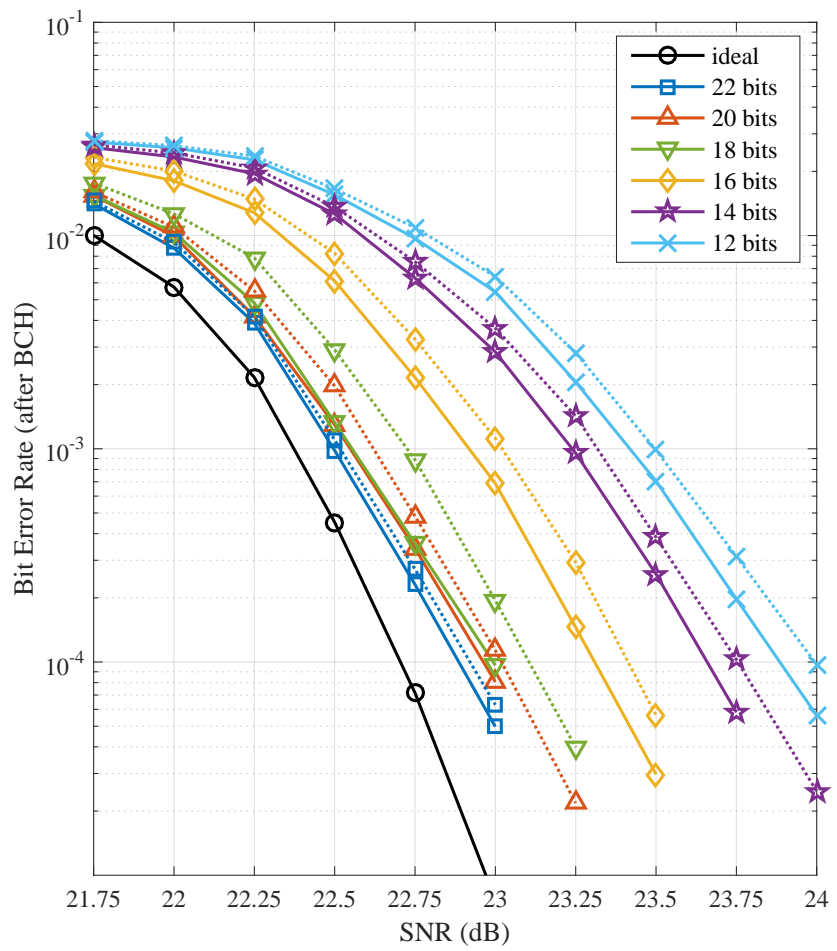

Figure 4.16: Performance evaluation of uniform (dashed lines) and non-uniform (continuous lines) LLR quantization, using 12 to 22 bits and compared to the non-quantized optimum performance, for $64 \mathrm{NUC}$ and CR 13/15.

\begin{tabular}{|c|c|c|c|c|c|c|}
\hline Quantization bits & $\mathbf{1 2}$ & $\mathbf{1 4}$ & $\mathbf{1 6}$ & $\mathbf{1 8}$ & $\mathbf{2 0}$ & $\mathbf{2 2}$ \\
\hline$\Lambda_{1}=\Lambda_{2}$ & 3 & 3 & 3 & 4 & 4 & 4 \\
\hline$\Lambda_{3}=\Lambda_{4}$ & 2 & 2 & 3 & 3 & 3 & 4 \\
\hline$\Lambda_{5}=\Lambda_{6}$ & 1 & 2 & 2 & 2 & 3 & 3 \\
\hline
\end{tabular}

Table 4.4: Number of bits per LLR for 64NUCs, depending on the total number of bits available.

quantize. The optimum non-quantized performance (black line) is compared with uniform (dashed lines) and non-uniform (continuous lines) quantization methods. In particular, the BER achieved with LLR quantization with several illustrative combinations is studied, i.e. different number of bits are used for each LLR, depending on the maximum reproducer obtained. Combinations of 2, 3 and 4 bits for each received LLR are considered, as shown in Table 4.4. 


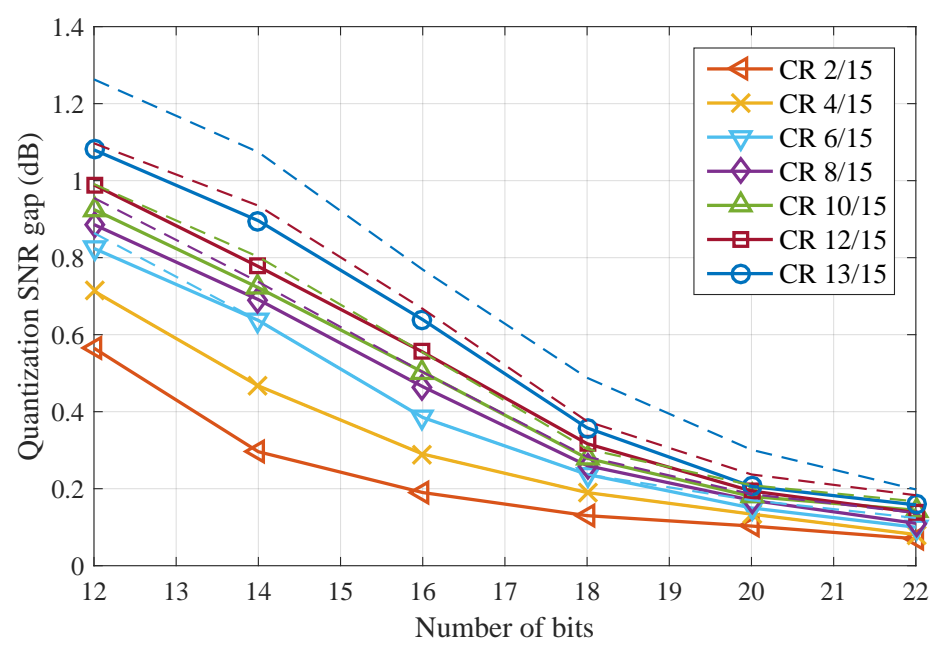

Figure 4.17: SNR gap of uniform (dashed lines) and non-uniform (continuous lines) LLR quantized $64 \mathrm{NUCs}$, using 12 to 22 bits.

\begin{tabular}{|c|c|l|l|l|l|l|l|l|}
\cline { 2 - 9 } \multicolumn{1}{c|}{} & CR (x/15) & $\mathbf{2}$ & $\mathbf{4}$ & $\mathbf{6}$ & $\mathbf{8}$ & $\mathbf{1 0}$ & $\mathbf{1 2}$ & $\mathbf{1 3}$ \\
\hline \multirow{3}{*}{ Uniform } & $\mathbf{1 6 N U C}$ & 10 & 11 & 12 & 12 & 12 & 13 & 14 \\
\cline { 2 - 9 } & $\mathbf{6 4 N U C}$ & 15 & 17 & 18 & 19 & 19 & 20 & 21 \\
\cline { 2 - 9 } & $\mathbf{2 5 6 N U C}$ & 21 & 23 & 24 & 25 & 26 & 28 & 30 \\
\hline \multirow{3}{*}{ Non-Uniform } & $\mathbf{1 6 N U C}$ & 10 & 11 & 11 & 12 & 12 & 12 & 13 \\
\cline { 2 - 9 } & $\mathbf{6 4 N U C}$ & 15 & 17 & 18 & 18 & 18 & 19 & 20 \\
\cline { 2 - 9 } & $\mathbf{2 5 6 N U C}$ & 21 & 22 & 23 & 24 & 25 & 26 & 28 \\
\hline
\end{tabular}

Table 4.5: Minimum number of uniform and non-uniform LLR quantization bits. SNR gap below $0.25 \mathrm{~dB}$.

As expected, the larger the number of bits the better the performance. Moreover, the difference between uniform and non-uniform quantization becomes almost negligible when using more bits, due to performance obtained, close to the optimum. Fig. 4.17 shows the SNR gap relative to the nonquantized case for a BER $=10^{-4}$, for all considered 64NUC and CR combinations. Non-uniform is always at least as good as uniform, with real gains at high CRs. For practical purposes, we postulate $0.25 \mathrm{~dB}$ as the maximum tolerable degradation due to quantization. This is a suitable value since the DVB-NGH channel model with speed $60 \mathrm{~km} / \mathrm{h}$ is used.

It is possible to provide a concrete number of bits $Q=\sum_{l=1}^{L} q_{l}$, with $L$ as the number of elements, to quantize each NUC and CR combination. The same 
CHAPTER 4. LOW-COMPLEXITY DEMAPPING AND QUANTIZATION ALGORITHMS

\begin{tabular}{|c|c|c|c|c|c|c|c|c|}
\hline Quantization bits & $\mathbf{1 6}$ & $\mathbf{1 8}$ & $\mathbf{2 0}$ & $\mathbf{2 2}$ & $\mathbf{2 4}$ & $\mathbf{2 6}$ & $\mathbf{2 8}$ & $\mathbf{3 0}$ \\
\hline$y_{1}=y_{2}$ & 4 & 5 & 6 & 6 & 7 & 7 & 8 & 8 \\
\hline$h_{1}=h_{2}$ & 4 & 4 & 4 & 5 & 5 & 6 & 6 & 7 \\
\hline
\end{tabular}

Table 4.6: Number of bits per I/Q component for $64 \mathrm{NUCs}$, depending on the total number of bits available.

procedure can be performed to $16 \mathrm{NUC}$ and $256 \mathrm{NUC}$ constellations. Table 4.5 summarizes the necessary number of bits $q$ for some representative CRs: $2 / 15$, $4 / 15,6 / 15,10 / 15,12 / 15$ and 13/15, considering both uniform and non-uniform quantization. One can affirm that $Q / m$ is almost independent of the constellation, e.g. $Q / m=3$ for non-uniform quantization of all NUCs at CR 8/15. This means that the number of quantizer bits per code bit is constellation-invariant and varies only little with $\mathrm{CR}$. Note that when a odd number of bits is selected, the assignment is done as shown in Table 4.4, with 1 bit of resolution subtracted from the least significant LLR.

\subsubsection{Performance Loss of I/Q Components Quantization}

In this section, the performance achieved with I/Q quantization is evaluated. Results are shown for $64 \mathrm{NUC}$ constellations, but the complete process has been repeated using $16 \mathrm{NUCs}$ and $256 \mathrm{NUCs}$. Fig. 4.18 shows the performance obtained for all NUC and CR combinations. Combinations of 4, 5, 6, 7 and 8 bits for each received real component are considered, as shown in Table 4.6.

With I/Q components, the number of bits used influences drastically the overall performance. Using 64NUCs designed for low CRs, just a low number of bits is required to quantize all signal components with a negligible SNR gap, due to strong and robust CR applied after the demapping process. However, using high CRs and keeping the same number of bits decreases the overall performance. Another interesting point is that non-uniform quantization always needs same or fewer bits to achieve the target performance. The same procedure can be extrapolated to $16 \mathrm{NUC}$ and $256 \mathrm{NUC}$ constellations. Table 4.7 summarizes the necessary number of bits $Q=\sum_{l=1}^{L} q_{l}$ for some representative CRs: $2 / 15,4 / 15,6 / 15,10 / 15,12 / 15$ and $13 / 15$, considering both uniform and non-uniform quantization. Comparing these results and results from Table 4.5, $\mathrm{I} / \mathrm{Q}$ quantization is worse than LLR quantization, except for $256 \mathrm{NUC}$ at CR $2 / 15,4 / 15$ and $6 / 15$. For this reason, only LLR quantizers are considered in next section. 


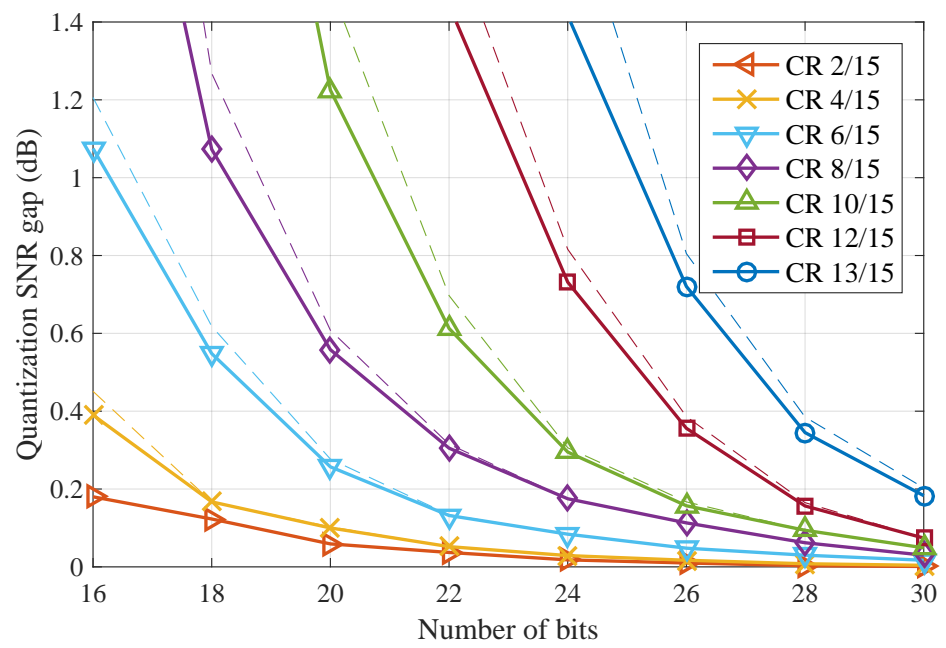

Figure 4.18: SNR gap of uniform (dashed lines) and non-uniform (continuous lines) I/Q quantized $64 \mathrm{NUCs}$, using 16 to 30 bits.

\begin{tabular}{|l|c|l|l|l|l|l|l|l|}
\cline { 2 - 9 } \multicolumn{1}{c|}{} & CR (x/15) & $\mathbf{2}$ & $\mathbf{4}$ & $\mathbf{6}$ & $\mathbf{8}$ & $\mathbf{1 0}$ & $\mathbf{1 2}$ & $\mathbf{1 3}$ \\
\hline \multirow{3}{*}{ Uniform } & $\mathbf{1 6 N U C}$ & 14 & 16 & 18 & 19 & 21 & 23 & 25 \\
\cline { 2 - 9 } & $\mathbf{6 4 N U C}$ & 15 & 17 & 20 & 23 & 25 & 27 & 30 \\
\cline { 2 - 9 } & $\mathbf{2 5 6 N U C}$ & 17 & 19 & 22 & 25 & 28 & 31 & 34 \\
\hline \multirow{3}{*}{ Non-Uniform } & $\mathbf{1 6 N U C}$ & 14 & 16 & 18 & 19 & 21 & 22 & 24 \\
\cline { 2 - 9 } & $\mathbf{6 4 N U C}$ & 15 & 17 & 20 & 23 & 25 & 27 & 30 \\
\cline { 2 - 9 } & $\mathbf{2 5 6 N U C}$ & 17 & 19 & 22 & 25 & 28 & 30 & 33 \\
\hline
\end{tabular}

Table 4.7: Minimum number of uniform and non-uniform I/Q quantization bits. SNR gap below $0.25 \mathrm{~dB}$.

\subsubsection{Time De-Interleaving Memory Requirements}

The total amount of memory needed at the TDIL, $\Delta$, can be calculated as expressed in Eq. 2.32, already introduced in Chapter 2, Section 2.3.2. In ATSC 3.0, maximum interleaving depths of $\Gamma=2^{19}$ for NUCs can be transmitted. Since $\Gamma$ is fixed, a reduction of $\Delta$ is in one-to-one correspondence to a reduction of $Q$, selected in previous sections.

Practical receivers use 6 bits per LLR [107], and 10 bits per I/Q component [28] in TDIL quantization. The total amount of memory used as reference when quantizing LLRs is 12.58, 18.87 and 25.17 Mbits for 16NUC, 64NUC and $256 \mathrm{NUC}$ respectively, while $20.97 \mathrm{Mbits}$ are required with I/Q components in all cases. For instance, from results presented in previous sections we can affirm 


\begin{tabular}{|c|c|c|c|}
\hline & Code Rate & $\begin{array}{c}\text { TDIL Memory } \\
\text { (Mbits) }\end{array}$ & $\begin{array}{c}\text { Reduction } \\
(\%)\end{array}$ \\
\hline \multirow{7}{*}{$16 \mathrm{NUC}$} & $2 / 15$ & 5.242 & 58.3 \\
\hline & $4 / 15$ & 5.762 & 54.2 \\
\hline & $6 / 15$ & 5.762 & 54.2 \\
\hline & $8 / 15$ & 6.291 & 50 \\
\hline & $10 / 15$ & 6.291 & 50 \\
\hline & $12 / 15$ & 6.291 & 50 \\
\hline & $13 / 15$ & 6.816 & 45.8 \\
\hline \multirow{7}{*}{$64 \mathrm{NUC}$} & $2 / 15$ & 7.864 & 58.3 \\
\hline & $4 / 15$ & 8.913 & 52.7 \\
\hline & $6 / 15$ & 9.437 & 50 \\
\hline & $8 / 15$ & 9.437 & 50 \\
\hline & $10 / 15$ & 9.437 & 50 \\
\hline & $12 / 15$ & 9.961 & 47.2 \\
\hline & $13 / 15$ & 9.961 & 47.2 \\
\hline \multirow{7}{*}{256 NUC } & $2 / 15$ & 8.913 & 57.5 \\
\hline & $4 / 15$ & 9.961 & 52.5 \\
\hline & $6 / 15$ & 11.534 & 45 \\
\hline & $8 / 15$ & 13.107 & 37.5 \\
\hline & $10 / 15$ & 13.107 & 37.5 \\
\hline & $12 / 15$ & 13.631 & 35 \\
\hline & $13 / 15$ & 14.681 & 30 \\
\hline
\end{tabular}

Table 4.8: Summary of required memory and reduction percentage with LLR quantization for all combinations considered.

that a $16 \mathrm{NUC}$ optimized for a CR 13/15 needs 13 bits to quantize all LLRs, as shown in Table 4.5. In this case, $\Delta=2^{19} \times 13$ bits, i.e. 6.81 Mbits are required, and compared to the 12.58 Mbits value, a $45.8 \%$ reduction of in-chip memory is achieved.

Comparing the results obtained in Table 4.5, and using Eq. 2.32, it is possible to determine the in-chip memory reduction, relative to the lowest quantization memory of reference (12.58, 18.87 and 20.97 Mbits for 16NUC, $64 \mathrm{NUC}$ and $256 \mathrm{NUC}$ respectively). Table 4.8 depicts the memory $\Delta$ required, and the reduction percentage achieved for each modulation and CR considered. Note that non-uniform quantization is always used, since it always provides same or better results than uniform. The higher the $\mathrm{CR}$ and modulation used the lower the reduction achieved. A reduction from $30 \%$ to $58.3 \%$, depending on the combination used, is obtained. 


\subsection{Conclusion}

In this chapter, the impact of NUCs on real receivers has been investigated from two different points, i.e. demapping of constellation symbols and quantization of I/Q components or LLR values obtained. Different algorithms have been also proposed as possible solutions to minimize the problems detected.

For the first case considered, a complete analysis on demapping complexity has been provided. The number of distances to calculate with QAM and 1DNUCs can be drastically reduced by using a 1D-demapper, as proposed in [26], and explained in Chapter 2. However, with 2D-NUCs and RCs, the demapper has to evaluate all distances in two dimensions. To reduce this complexity, a suboptimum demapper called QCSR has been proposed. The demapper is based on the combination of two strategies to take advantage of the symmetry of the constellations and the condensation, i.e., similar I/Q values of several constellation symbols, of NUCs at low SNRs. An example of application has been shown for $2 \mathrm{D}-256 \mathrm{NUCs}$. A reduction in the number of required operations from $69 \%$ to $93 \%$ has been achieved, depending on the CR, when compared to the optimum ML demapper, with almost no performance degradation (under $0.1 \mathrm{~dB})$. The demapper can be extrapolated to the different orders of constellation considered in this thesis, i.e. 2D-16NUC and 2D-64NUC, or even larger constellations such as $2 \mathrm{D}-1 \mathrm{kNUC}$ or $2 \mathrm{D}-4 \mathrm{kNUC}$. It can be applied to rotated constellations as well, without any complexity or performance implication.

The influence of NUCs on digital quantizers has been also studied. A different shape implies different distributions for the I/Q components and the LLRs obtained after the demapping process. To reduce the amount of bits to store at the TDIL, a new strategy based on BICM capacity maximization via the Nelder-Mead algorithm has been proposed. It has been applied to both I/Q components and LLR values, selecting the best case for each modulation and $\mathrm{CR}$ combination, and providing memory reduction percentages compared to conventional quantizers. In general, non-uniform LLR quantization is the best choice in the sense of meeting the performance target with minimum memory requirements. The use of non-uniform quantizers adapted to the signal statistics provide slight improvements in terms of performance or alternatively in-chip memory savings. For the worst case considered, i.e. $256 \mathrm{NUC}$ with CR 13/15, a final reduction in TDIL memory of 30\% compared to conventional quantizers has been achieved, when quantizing LLR values. Better results have been obtained for low-order constellations. With 16NUCs, the total memory reduction ranges from $45.8 \%$ to $58.3 \%$. For 64 NUCs, reduction percentages from $47.2 \%$ to $58.3 \%$ have been also obtained. 
CHAPTER 4. LOW-COMPLEXITY DEMAPPING AND QUANTIZATION ALGORITHMS 


\section{Chapter 5}

\section{Non-Uniform \\ Constellations for MIMO Communications}

The use of non-uniform constellations in single-antenna broadcast communications has been studied in Chapters 3 and 4 from different transmit and receive points of view, respectively. This includes the optimization process, performance analysis and complexity (demapping and quantization). With MIMO, both transmit and receive systems are modified, since multiple antennas are used. The use of several antennas clearly affects both the channel model and the SNR range in which the constellations work. Constellations optimized for SISO may not be optimum for MIMO, as proposed in the ATSC 3.0 standardization process. In addition, further parameters need to be considered: power imbalance (PI), cross-polar discrimination (XPD), number of transmit and receive antennas, or correlation factor. In this thesis, two transmit and receive cross-polar antennas are assumed. The influence of these parameters as well as the difference between SISO and MIMO configurations is studied in Sections 5.1 and 5.2 .

At the receiver, one of the most important drawbacks is the extremely high demapping complexity when using NUCs and optimum ML or max-log demappers. Section 5.3 provides a complete demapping complexity analysis, which shows that the number of possible received symbols grows exponentially with the number of antennas and the constellation order. To reduce this complexity, different sub-optimum demappers can be used. In Section 5.4, the particular case of the SFSD demapper is analyzed. It entails a good trade-off between 


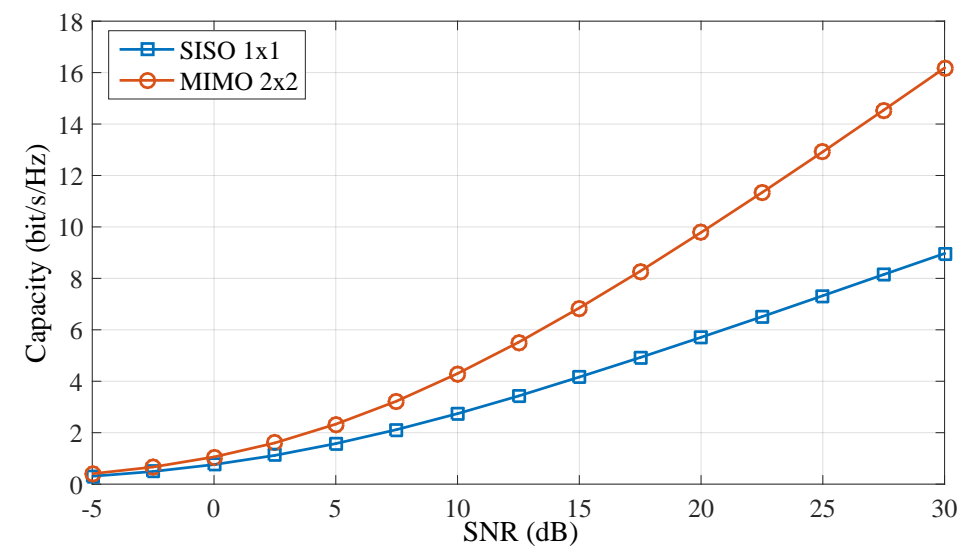

Figure 5.1: Ergodic capacity for a mobile DVB-NGH channel model, with user speed 60 $\mathrm{km} / \mathrm{h}$, and same transmit power in the two cases.

performance and demapping complexity. With SFSD, it is necessary to perform a quantization step that needs to be performed in separated I and Q components [80]. Thanks to the squared shape of QAM and 1D-NUCs, both components can be separated. However, this is not possible with 2D-NUCs. In this chapter, and an efficient pre-processing approach for 2D-NUCs based on the Voronoi regions for the quantization step is proposed.

\subsection{Multi-Antenna Non-Uniform Constellations}

The implementation of MIMO provides three different types of gains: array gain, diversity gain, and multiplexing gain [75]. The array gain comes from the coherent combination of the received signals from the multiple antennas. For co-polar antennas, this gain equals $3 \mathrm{~dB}$ every time the number of antennas is doubled. The diversity gain is due to the fact of having several spatial branches with independent fading between the transmitter and receiver. And the multiplexing gain allows transmitting more than one data stream [76]. Fig. 5.1 shows the ergodic capacity for SISO and MIMO with two transmit and two receive antennas, in the mobile DVB-NGH channel (user speed $60 \mathrm{~km} / \mathrm{h}$ ) [33].

The MIMO capacity increases with the SNR at a higher rate than the SISO scheme. This difference in spectral efficiency is due to multiplexing gain for a MIMO channel employing two transmit and receive antennas with independent streams. The gain depends also on the particular channel. For the analyzed case, increased diversity due to multiple antennas at low SNR levels provides 
very significant gains in relative terms. The DVB-NGH mobile model gives a capacity gain (vertical axis) compared to SISO of $40 \%, 48 \%$ and $57 \%$, for 0 , 5 and $10 \mathrm{~dB}$ SNR, respectively. Fig. 5.1 also depicts the difference in SNR between the two configurations utilized. For instance, in order to achieve a channel capacity of $4 \mathrm{bit} / \mathrm{s} / \mathrm{Hz}$, and using just a single antenna in both transmit and receive sides, a minimum SNR of approximately $15 \mathrm{~dB}$ is necessary. With $2 \times 2$ MIMO systems, the SNR required is reduced to $10 \mathrm{~dB}$. Therefore, there is a difference in performance of $5 \mathrm{~dB}$ between both configurations. This difference logically influences the optimization process, since the SNR target for which the NUC is designed totally changes. However, not only the use of two receive and transmit antennas modifies the constellation shape. As already mentioned, more parameters may change the final result when optimizing NUCs for MIMO. Capacity and performance gains over QAM obtained need to be analyzed and compared with those obtained in SISO as well.

In this section, we follow a similar structure than Section 3.2 in Chapter 3. Two different optimization techniques are investigated and particularized for MIMO, depending on the number of real-valued dimensions considered in the optimization process: 1D- and 2D-NUCs. Since constellations optimized in this chapter are compared with those optimized for SISO in Chapter 3, it is necessary to differentiate them with a new notation. A constellation optimized for $2 \times 2$ MIMO configurations is called in this dissertation Multi-Antenna Non-Uniform Constellation (MA-NUC). Constellations optimized for one and two dimensions are then called 1D-MA-NUC and 2D-MA-NUC, respectively. On the other hand, constellations optimized for SISO but used in MIMO are referred just as 1D-NUC and 2D-NUC. The optimization process of both 1DMA-NUC and 2D-MA-NUC is explained in following subsections.

\subsubsection{Preliminary Design}

In this section, we investigate in detail the concrete parameters that affect the optimization process by the use of two transmit and receive antennas. In particular, three different subsections are considered. First, the optimization of NUCs in one or two antennas is analyzed. The first option maximizes the mutual information in a single antenna, optimizing the constellation transmitted in the first antenna and replicating the same result in the second antenna. The second option optimizes both constellations independently, achieving better capacity results at the expense of a higher optimization complexity. This becomes especially effective when considering a PI between antennas, where a different SNR is required in each antenna. The second subsection studies the demapping influence on constellations, and selects the best option in terms of performance and complexity. Finally, several channel models are analyzed. 


\section{CHAPTER 5. NON-UNIFORM CONSTELLATIONS FOR MIMO COMMUNICATIONS}

\section{Optimization in 1 or 2 Antennas}

Traditional $2 \times 2$ MIMO systems that use a symmetric modulation in both transmit antennas utilize $2 \log _{2}(M)$ bits per cell, where $M$ is the number of symbols in a single antenna. In such case, and using uniform QAM constellations, the half of the bits is transmitted in one antenna, while the other half is used in the other, using identical constellations in both of them. For example, 12 bits per cell means that 6 bits are associated to each antenna, where 64 symbols are used.

Considering no power imbalance between antennas, one could think that such symmetry must be maintained with MA-NUCs. However, the optimization process allows us to explore new possibilities. The first option is simply to optimize the constellation transmitted in the first antenna, replicating and transmitting it in the second antenna as well. The parameters to configure are still grouped in a single vector $\mathbf{a}=\left[a_{1}, a_{2}, \ldots, a_{N}\right]$, with $N$ as the degrees of freedom in the optimization, which depends on the constellation order. Compared to SISO, the optimization burden is maintained. The second option is to optimize both constellations by separate, identifying two different and independent vectors:

$$
\left\{\begin{array}{l}
\mathbf{a}_{\mathbf{1}}=\left[a_{11}, a_{12}, \ldots, a_{1 N}\right] \\
\mathbf{a}_{2}=\left[a_{21}, a_{22}, \ldots, a_{2 N}\right]
\end{array}\right.
$$

where the first index indicates the transmit antenna, and the second term represents the position of the constellation symbol under study. In this section, an i.i.d. Rice channel with $K=10$ (as defined in Appendix A) is considered, since it is a model used for cross-polarized MIMO with fixed rooftop reception and LoS. The main idea is to observe the similarities and differences introduced by the channel to the two optimization options already considered, but also to take into account the new parameters that influence the process such as PI or XPD. Fig. 5.2 shows the evolution of the two parameters $a_{11}$ and $a_{21}$ when optimizing each 1D-MA-16NUC constellation independently for a SNR of 20 $\mathrm{dB}$, without PI. Three different XPD values are also evaluated: 0,6 and $\infty$ dB.

As Fig. 5.2 shows, both $a_{11}$ and $a_{21}$ parameters evolve towards the same (or almost) final value, regardless of the cross-polar discrimination introduced. The XPD at the receiver can be calculated as [108]:

$$
\mathrm{XPD}_{1}=\frac{\left|h_{11}\right|^{2}}{\left|h_{21}\right|^{2}}
$$



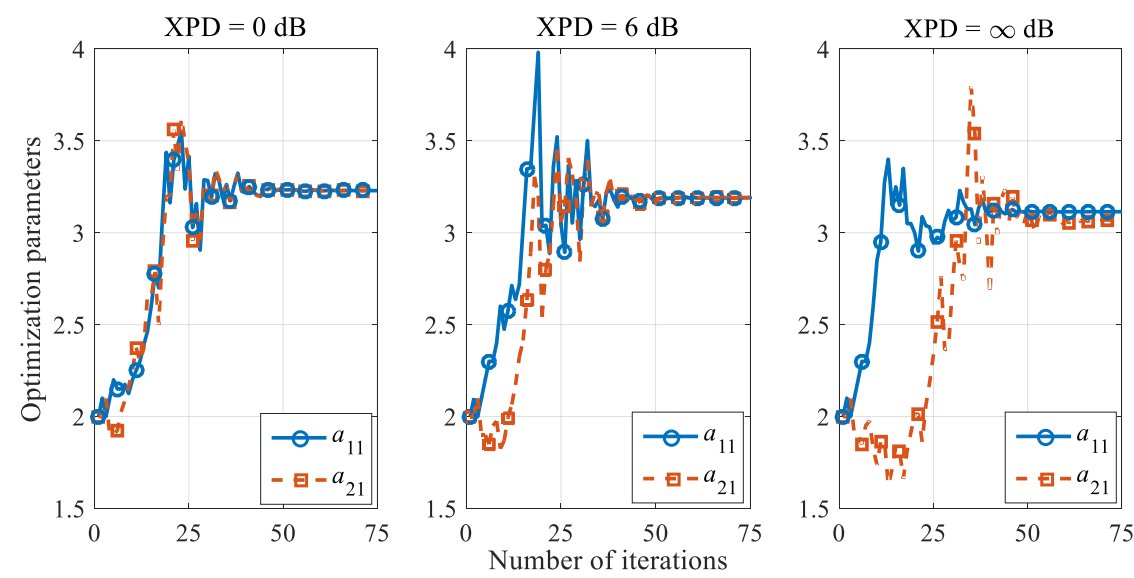

Figure 5.2: Evolution of the optimization parameters $a_{11}$ and $a_{21}$, for 16-MA-NUCs, SNR $20 \mathrm{~dB}$ and three different XPD values: 0,6 and $\infty \mathrm{dB}$.

$$
\mathrm{XPD}_{2}=\frac{\left|h_{22}\right|^{2}}{\left|h_{12}\right|^{2}}
$$

The term $\mathrm{XPD}_{1}$ denotes the average power of the co-polar term (direct path) for the first antenna divided by the average power of the cross-polar term, introduced to the incorrect reception antenna, in this case the second one. In addition, both terms need to fulfill that $\sum_{i}^{2}\left|h_{i, j}\right|^{2}=1$. Note that the channel is symmetrically generated:

$$
\mathrm{XPD}_{1}=\mathrm{XPD}_{2}
$$

Thus, an XPD $=6 \mathrm{~dB}=4$ (lineal scale) determines a channel matrix in which the co-polar terms are transmitted with average power $\left|h_{11}\right|^{2}=\left|h_{22}\right|^{2}=$ 0.8 , and cross-polar terms with $\left|h_{21}\right|^{2}=\left|h_{12}\right|^{2}=\frac{0.8}{4}=0.2$. For the extreme case where $\mathrm{XPD}=0 \mathrm{~dB}=1$ (lineal scale), all terms have average power 0.5. On the other hand, with XPD $=\infty$, both cross-polar terms are zero, while direct paths $\left|h_{11}\right|^{2}=\left|h_{22}\right|^{2}=1$. For this reason, optimized values in Fig. 5.2 are very similar for both antennas, due to the expression 5.4. Without PI, both constellations tend towards the same final result, since received signals in the two receive antennas have on average the same power. The XPD value also affects the optimized parameters obtained. Larger XPD values affect less the two signals at the receive antennas, requiring symbol positions as uniform as possible (they tend to uniform QAM). 


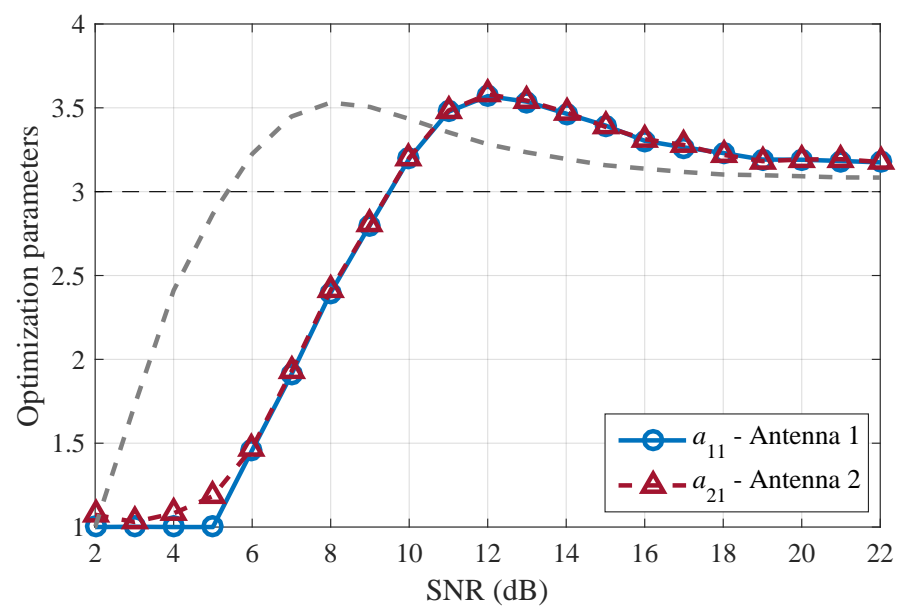

Figure 5.3: Optimization parameters $a_{11}$ and $a_{21}$ for 1D-MA-16NUCs as a function of the SNR, i.i.d. Rice channel with $K=10$.

Fig. 5.3 shows the whole SNR range for the 1D-MA-16NUCs considered in the two different antennas, for i.i.d. Rice channel and XPD $=6 \mathrm{~dB}$. Based on these results, from now it is assumed $\mathbf{a}_{\mathbf{1}}=\mathbf{a}_{\mathbf{2}}=\mathbf{a}$ for $\mathrm{PI}=0 \mathrm{~dB}$, i.e. the same constellation is used in both antennas. This assumption logically cannot be taken when using PI. Fig. 5.3 also shows the difference between SISO and MIMO. There is a clear shift in SNR between both results, mainly because of the $3 \mathrm{~dB}$ coming from the array gain and the cross-polarized terms at the receiver. The fact that constellations adapt dynamically to the number of antennas and XPD means that NUCs optimized for SISO can be used for MIMO as well, and vice versa. NUCs that are designed for SISO and for a specific modulation and CR combination can still be used for MIMO, for the same configuration. This assumption was already taken in the ATSC 3.0 specification [76], and will be proved in Section 5.2, where performance results are provided.

\section{Influence of ML, ZF and MMSE Demappers}

In this section, we study the influence of the type of demapper used on the optimized NUCs. Although the ML demapper obtains optimum performance, it entails a high demapping complexity that increases with the order of constellation and number of antennas. In this sense, several equalization methods such as ZF or MMSE can be used to reduce the complexity. The use of these 

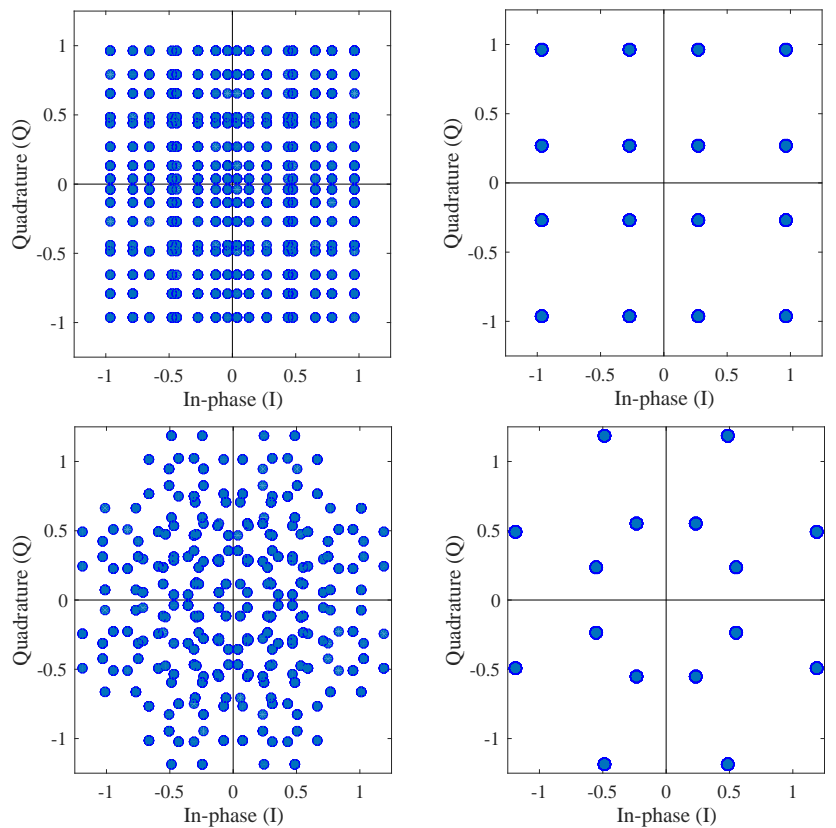

Figure 5.4: Examples of 1D- and 2D-16NUCs at the receiver input, before (left) and after (right) equalization, noise-free reception.

equalizers reduces enormously the demapping complexity, accelerating the optimization process and allowing to optimize very high-order constellations (ML with MIMO is not computationally feasible from $1 \mathrm{kNUC}$ on). In fact, many real receivers implement this technology to facilitate real-time demodulation. Note that sphere decoders can be used as well to reduce complexity. However, they are not considered in this section since their use is not straightforward. $\mathrm{SD}$ is explained in Section 5.4.

The demapping schemes considered when using MMSE and ZF equalizers can be found in Chapter 2. An optimum ML demapper computes LLRs for all code bits $2 B$, where $B$ refers to the number of bits per symbol used in a single antenna. For the LLR computation, a total number of $M^{2}$ Euclidean distances between the received vector $\mathbf{y}$ and constellation symbols $\mathbf{x}$ is calculated. The received constellations in the two antennas present the following expression:

$$
\left\{\begin{array}{l}
y_{1}=h_{11} x_{1}+h_{12} x_{2} \\
y_{2}=h_{21} x_{1}+h_{22} x_{2}
\end{array}\right.
$$




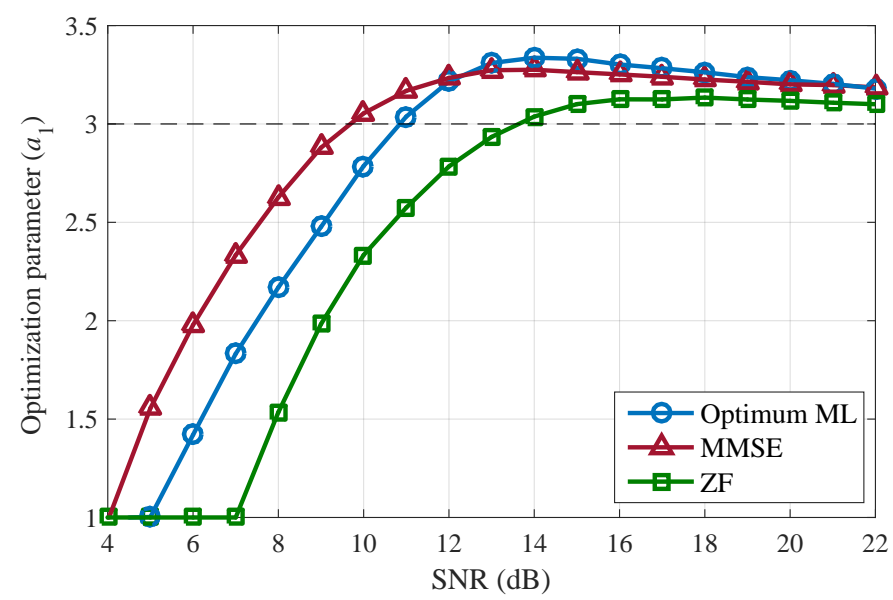

Figure 5.5: Optimization parameter $a_{1}$ for 1D-MA-16NUCs and different MIMO demappers, i.i.d. Rayleigh channel. Results are also compared to SISO.

As a consequence, the received constellation in each antenna is a linear combination of both $x_{1}$ and $x_{2}$, which depends on the XPD introduced. On the other hand, ZF and MMSE linear equalizers transform the joint MIMO demapping process into independent single-antenna demapping blocks. In a $2 \times 2$ MIMO system, the ML demapper with complexity $M^{2}$ is transformed into two independent ML demappers with complexity $M$. To do this, the cross components of the channel $\left(h_{12}, h_{21}\right)$ are suppressed, providing two estimates $\tilde{y}_{1}$ and $\tilde{y}_{2}$ of the received symbol. LLRs are then calculated and combined in a single stream. Fig. 5.4 shows an example of 1D- and 2D-NUCs at the receiver input for the first antenna, before (left) and after (right) equalization with MMSE. Noise-free reception is assumed to better show the resulting constellations, where $\mathbf{H}=\mathbf{I}$ and XPD $=6 \mathrm{~dB}$.

Naturally, the use of linear equalizers distorts the received signal, which turns into slightly different optimization values. Fig. 5.5 shows the $a_{1}$ value optimized for a 1D-MA-16NUC and a complete SNR range, for the different demappers considered above. It is assumed that the same constellation is transmitted over the two antennas. When ZF is used, the receive signal is equalized making use of the channel matrix, not considering the noise introduced, as expressed in Eq. 2.29, in Chapter 2. As a consequence, the noise is amplified, which transforms the optimized constellation into a more condensed one with lower $a_{1}$ value. A different behavior can be seen when using MMSE. In this case, the noise power is included, making the $a_{1}$ value more similar to ML, 


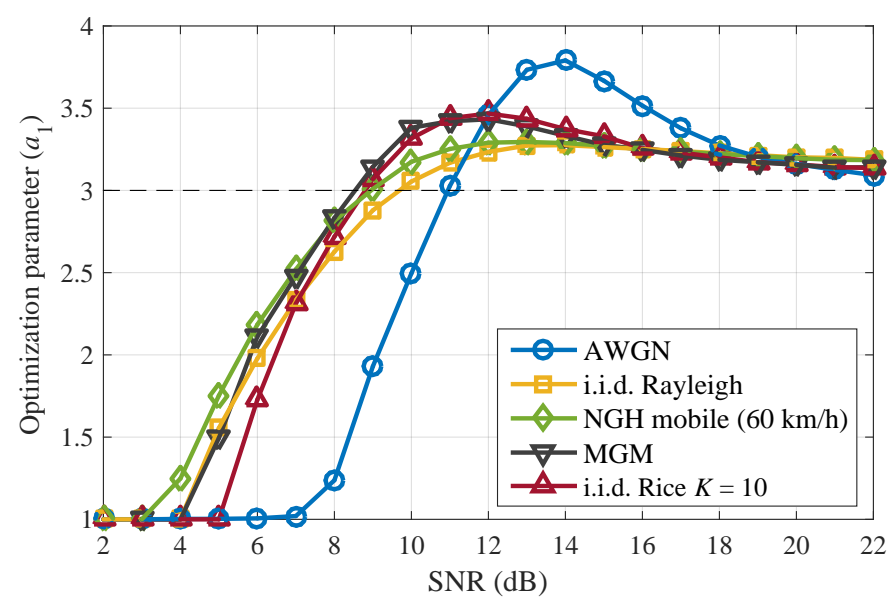

Figure 5.6: Optimization parameter $a_{1}$ for 1D-MA-16NUCs and different channel models: AWGN, i.i.d. Rice, mobile DVB-NGH and MGM.

especially at high SNRs where this type of equalizer works better. On average, the difference between $\mathrm{ML}$ and $\mathrm{ZF}$ in the final optimization parameter, for the whole considered SNR range, is 0.23 (distance in a single component), while the difference between ML and MMSE is reduced to 0.11. From this point, we assume an MMSE equalizer for this section.

\section{Selection of Channel Model}

As already mentioned, optimization parameters not only depend on the SNR, but also on the channel model. Fig. 5.6 shows the parameter $a_{1}$ obtained for five different channel models: AWGN, i.i.d. Rayleigh, i.i.d. Rice with line-ofsight $K=10$, the mobile DVB-NGH channel with user speed $60 \mathrm{~km} / \mathrm{h}$ and the Modified Guildford Model (MGM) without log-normal fading. Results show a similar tendency than the obtained for SISO (see Fig. 3.3). In AWGN, the parameter has its peak value at around $14 \mathrm{~dB}$ SNR, and the condensation is maintained for a wider range of low SNRs, compared to other channels. In i.i.d. Rayleigh, the curve gets smoother, keeping a similar value over the whole SNR range from 11 to $22 \mathrm{~dB}$. Despite the Doppler introduced $(33.3 \mathrm{~Hz}, 60 \mathrm{~km} / \mathrm{h})$, a similar behavior can be observed with the DVB-NGH mobile channel, as well as with MGM. The result with i.i.d. Rice is a middle term between AWGN and i.i.d. Rayleigh, since the channel provides better estimations due to the LoS $(K=10)$, but still introduces fading. We use the i.i.d. Rice channel in next sections. 

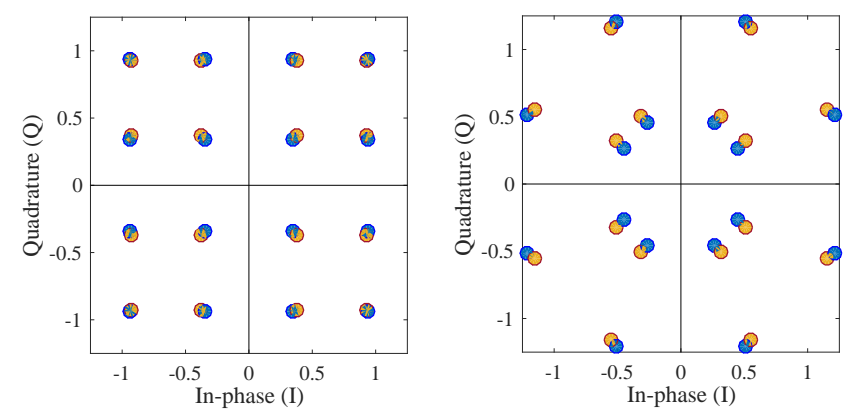

Figure 5.7: Different 16NUCs optimized for i.i.d. Rice channel and CR 4/15. Left part: 1D-NUC (blue) vs. 1D-MA-NUC (orange). Right part: 2D-NUC (blue) vs. 2D-MA-NUC (orange).

\subsubsection{Re-optimization without Power Imbalance}

In Chapter 3, constellations were designed seeking the maximum BICM capacity for a large range of SNRs. Afterwards, the target SNR of each particular NUC was selected for a CR according to the SNR of the waterfall region. For MIMO, we follow a different methodology. Constellations designed for SISO are not optimum for MIMO anymore, since they were designed for a different SNR and channel model. However, they still keep a good capacity gain, due to the shifting produced when doubling the number of antennas. Note that, in this dissertation, the same transmission power used in SISO is split into the two antennas. When doubling the transmission power, the result changes. In following examples, NUCs optimized for specific CRs and SISO are taken as initial parameters to optimize. A re-optimization process is then performed, where the SNR of the waterfall region is again selected considering a $2 \times 2$ MIMO scheme.

In this section, $16 \mathrm{NUCs}$ are shown as an example of application. When transmitting the same uniform 16QAM constellation in both antennas, and using a CR 4/15, the minimum SNR necessary to achieve a BER $\leq 10^{-4}$ in i.i.d. Rice channel is $7.6 \mathrm{~dB}$. This value is taken as the SNR target for optimization. If a 1D-16NUC is used instead of QAM, which was already optimized for SISO (where the SNR target for i.i.d. Rice channel is $2.7 \mathrm{~dB}$ ), the BICM capacity is practically maintained. The optimization parameter for SISO is $a_{1}=2.72$. Based on this parameter, it is possible to re-optimize the constellation considering all configurations selected in previous sections, e.g. same constellation in both antennas and the use of a MMSE demapper. The obtained 1D-MA16NUC constellation slightly increases the BICM capacity, and modifies the optimum parameter $a_{1}=2.48$. However, the BICM capacity increase is not 


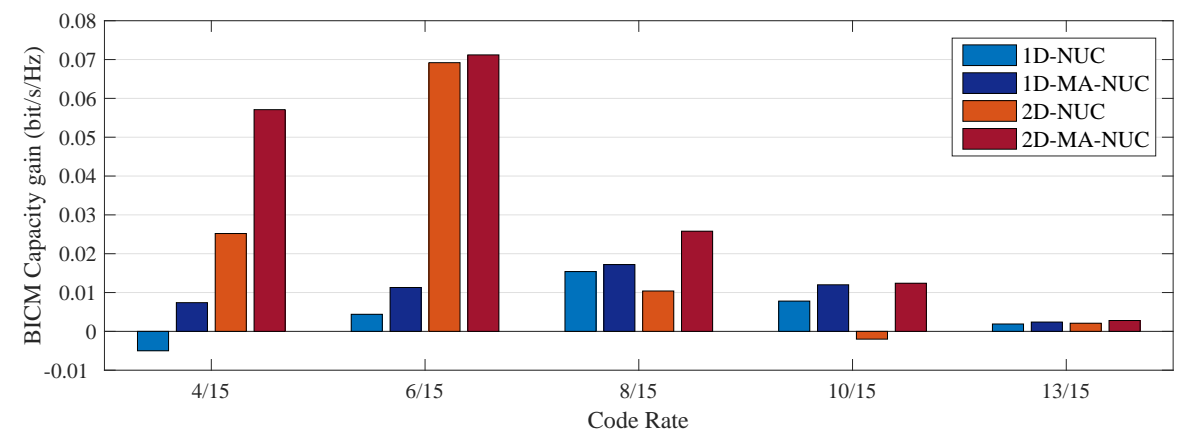

Figure 5.8: BICM capacity gain of 1D/2D-16NUC and 1D/2D-MA-16NUC over QAM, for i.i.d. Rice channel model and five representative CRs.

significant, since the DoF with these constellations is just one, simplifying the optimization process. With 2D-NUCs, there are more DoF to optimize, and therefore the capacity increase achieved is higher. A re-optimized 2D-NUC in this case achieves additional improvements, but still not significant. Fig. 5.7 depicts the different 1D- and 2D-MA-NUCs designed (orange), compared to the original NUCs from SISO (blue). As Fig. 5.7 shows, similar constellations are obtained despite the re-optimization.

Fig. 5.8 shows the BICM capacity gains with respect to QAM for the four different 16NUCs considered: 1D-NUC, 2D-NUC, 1D-MA-NUC and 2D-MANUC. Five representative CRs are selected from ATSC 3.0 [15]: 4/15, 6/15, $8 / 1510 / 15$ and $13 / 15$. The CR $2 / 15$ is not evaluated, since the same capacity gain is achieved for all constellations. At low SNRs, 16NUCs tend towards QPSK, regardless of the number of dimensions in which the optimization is done. The same does not occur with the rest of cases explored. For medium CRs, two different considerations can be made. First, the MA-NUC capacity gain over single-antenna NUCs is always higher, but almost negligible. Second, the $2 \mathrm{D}$ gain over $1 \mathrm{D}$ is also higher. This already happened in SISO, where 2DNUCs changed towards a Gaussian shape that approaches better the channel characteristics. For high CRs, the capacity gains obtained are tiny. Coding tends to unity where no redundancy bits are employed. In this case, uniform separations between symbols ensure the minimum distances between them, minimizing the errors as much as possible, so the best possible constellation is similar to QAM. In general, one can affirm that the capacity gains achieved are not significant, and turn into slight performance gains, as shown in Section 5.2 . 

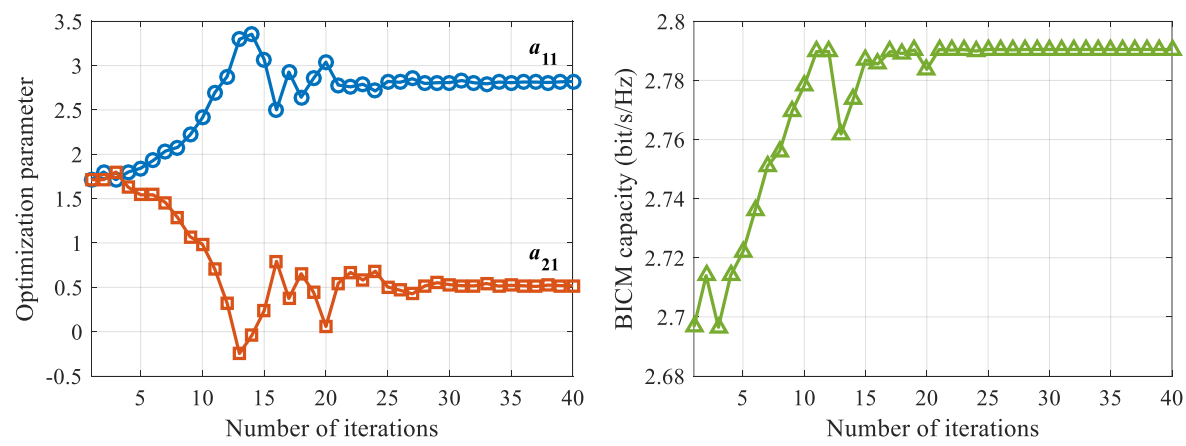

Figure 5.9: Optimization parameters for the two 16NUCs transmitted using a CR 4/15, and a PI of $6 \mathrm{~dB}$. Initial parameters from SISO.

\subsubsection{Re-optimization with Power Imbalance}

In this section, the same re-optimization process is applied but with a PI between the two streams transmitted. An imbalance between both streams can be intentionally generated in transmission by operators to facilitate the use of dual polar MIMO operations in specific networks [76]. For this study, we modify the power allocated at each transmit antenna as detailed in Chapter 2. PI is included as part of the channel to provide a generic approach that takes into account the intentional imbalances introduced at the transmitter. From the receiver point of view, both transmitted streams experience different SNRs, thus requiring different asymmetric NUCs and using different optimization parameters $a_{11}$ and $a_{21}$.

Fig. 5.9 shows the re-optimization process for the two 1D-16NUCs transmitted using a CR 4/15, and a PI of $6 \mathrm{~dB}$. The SNR target considered for i.i.d. Rice channel and a CR $4 / 15$ is $7.6 \mathrm{~dB}$. Fig. 5.9 clearly depicts how both parameters follow different tendencies, almost symmetric, which also result in distinct optimized constellations. Note that the parameter obtained for the second antenna acquires a value $a_{21}=0.5$, lower than the unity. That means that the resulting constellation symbols in a single I/Q component are exchanged. Therefore, bits are mapped to symbols in a different way than the first antenna, which employs a regular Gray-mapping. The resulting constellations in both antennas for CR 4/15, and using 1D and 2D optimization, are shown in Fig. 5.10. It can be observed that more condensed constellations are obtained for the second MIMO stream, which has lower signal power.

Fig. 5.11 depicts the BICM capacity gains with respect to QAM for a PI of $6 \mathrm{~dB}$. The same representative CRs are used. As occurred without PI, the 

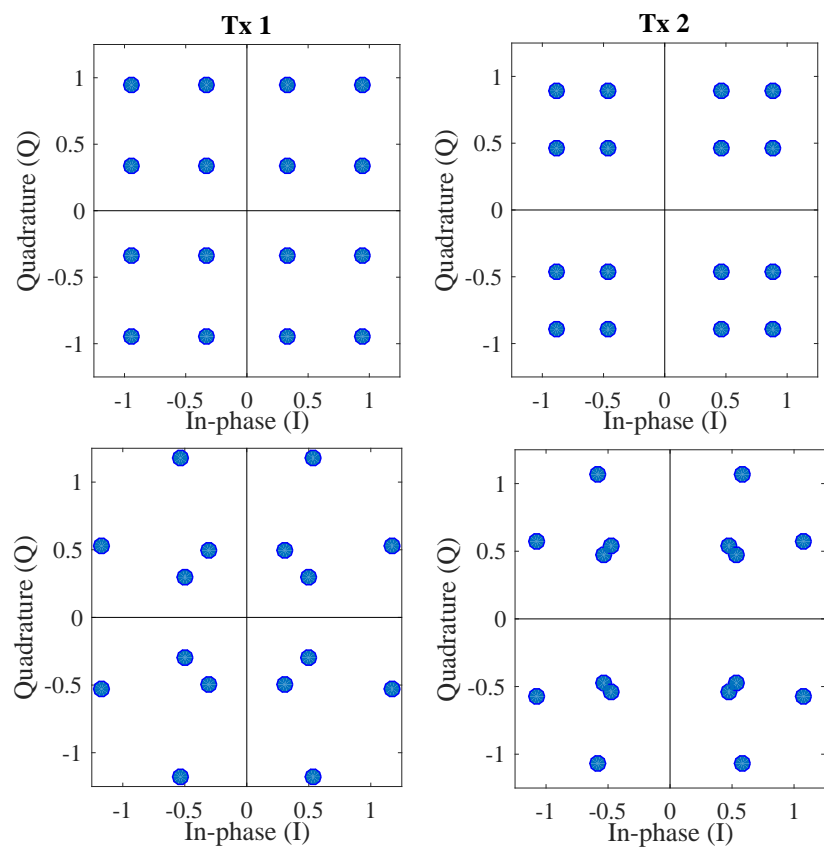

Figure 5.10: Different 16-MA-NUCs optimized for i.i.d. Rice channel and CR 4/15. 1D-NUC (top) and 2D-NUC (bottom) are shown for both transmitters.

same capacity gain and therefore the same resulting NUCs are achieved for a CR 2/15. Although different transmission powers are considered, both antennas still experience very low SNRs, tending towards the same condensed MA-NUC. The highest gain is obtained for a CR 4/15. The use of a different MA-NUC per antenna that is adapted independently to each stream, and therefore to the power imbalance introduced, increases significantly the capacity compared to regular NUCs. SISO constellations that do not consider the power imbalance condition and are optimized for a different SNR can even introduce an additional loss. This is the case when using 1D-NUCs for the CR 4/15 or 2D-NUCs for the CR 10/15. For high CRs such as 13/15, the capacity gains obtained are negligible, with similar MA-NUCs obtained for each antenna. Compared to the optimization without PI, no difference is observed for this specific CR. 


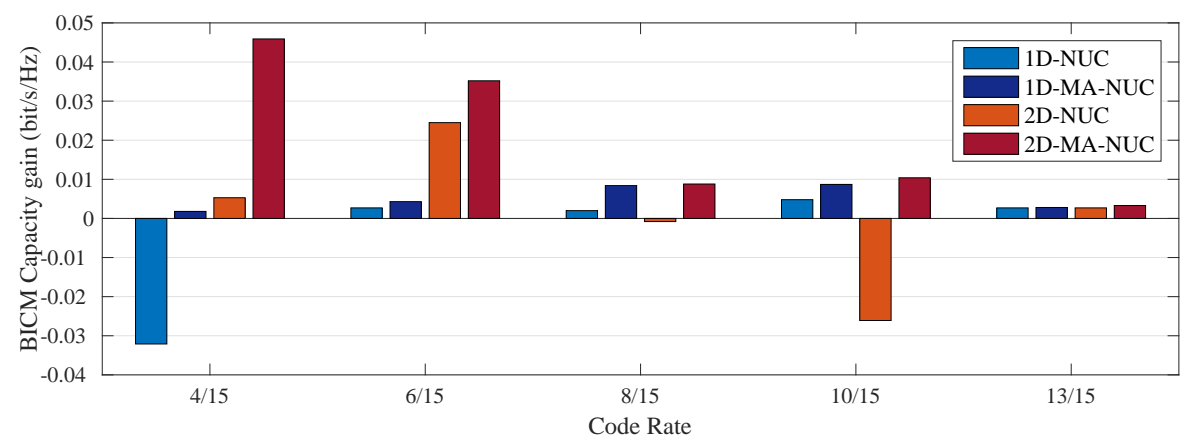

Figure 5.11: BICM capacity gain of 1D/2D-NUC and 1D/2D-MA-NUC over QAM, for i.i.d. Rice channel model and five representative CRs, $\mathrm{PI}=6 \mathrm{~dB}$.

\subsection{Performance Evaluation of Multi-Antenna Non-Uniform Constellations}

\subsubsection{Transmission without Power Imbalance}

The design and implementation of MA-NUCs only affect the mapping and demapping processes. In this section, the ATSC 3.0 specification was used for the rest of blocks implemented in both transmitter and receiver. NUCs for SISO, optimized in Chapter 3 are also used for comparison. MMSE equalization followed by two ML demappers was considered in all cases. A LDPC code length of 64800 bits was used, with bit and frequency interleavers activated [15]. Ideal channel estimation and an XPD of $6 \mathrm{~dB}$ are also considered. The complete transmit to receive simulation chain is defined in Appendix A.

Fig. 5.12 presents the performance achieved using the 1D- and 2D-MA16NUCs designed for an i.i.d. Rice channel without PI. Fig. 5.12 also shows the performance of those constellations that initially were designed for the same $\mathrm{CR}$ and channel model, but for a SISO configuration. Two main conclusions can be extracted from this figure. First, 2D-NUCs always obtain same or better gains than 1D-NUCs. This result is very well aligned with the BICM capacity gains obtained in Section 5.1. As occurred with SISO, 2D-optimization permits the symbols a higher freedom to reach their optimum position, thus obtaining higher gains. Second, we can affirm that MA-NUCs do not provide significant gains compared to original NUCs for this case, due to the array gain considered in the optimization process when doubling the number of antennas. MIMO transmissions without PI require the same constellation in both antennas, since both streams experience similar channel conditions. Therefore, constellations 


\subsection{Performance Evaluation of Multi-Antenna Non-Uniform}

Constellations

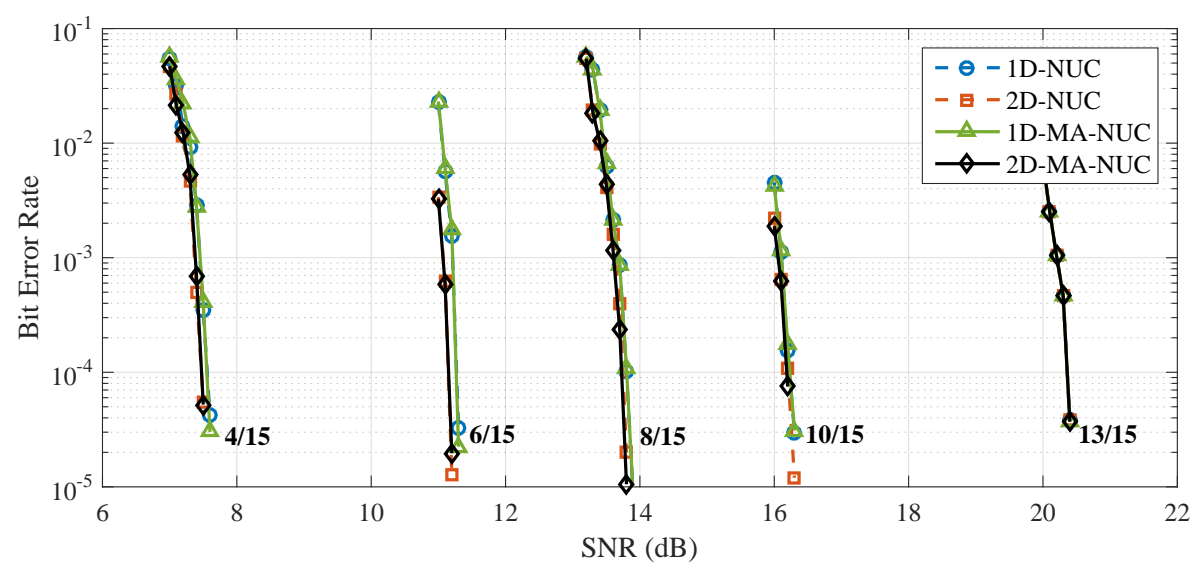

Figure 5.12: Performance (dB) of 1D/2D-16NUC and 1D/2D-MA-16NUC, for i.i.d. Rice channel model and five representative $\mathrm{CRs}, \mathrm{PI}=0 \mathrm{~dB}$.

optimized for SISO are almost optimum for MIMO as well. Note that the performance gains over QAM are very similar to those obtained in SISO.

\subsubsection{Transmission with Power Imbalance of $6 \mathrm{~dB}$}

MA-NUCs are effective only when exploiting a particular PI between antennas, and for specific CRs. Fig. 5.13 shows an example for a PI of $6 \mathrm{~dB}$ and a CR 4/15. As can be observed, NUCs optimized for SISO do not give any performance gain compared to QAM. In fact, the 1D-NUC used entails a performance loss higher than $0.1 \mathrm{~dB}$. A re-optimization that considers the PI permits to adapt the constellations to the new channel, obtaining an additional gain.

Gains provided for the representative CRs can be found in Fig. 5.14. This result is well-aligned with the capacity results obtained in Section 5.1.3. The use of a different 2D-MA-NUC per antenna that is adapted independently to each stream, and therefore to the power imbalance introduced, reduces in approximately $0.1 \mathrm{~dB}$ the minimum SNR required, compared to regular 2DNUCs. In general, results are aligned with the BICM capacity results. For instance, a performance loss is introduced when using 1D-NUCs for the CR 4/15 or 2D-NUCs for the CR 10/15, since SISO constellations do not consider the power imbalance condition. For high CRs such as 13/15, the capacity gains obtained are very low, and do not depend on the use of MA-NUCs or single-antenna NUCs. Compared to the performance results without PI, lower SNR gains are obtained, but the use of MA-NUCs is effective for some CRs, 


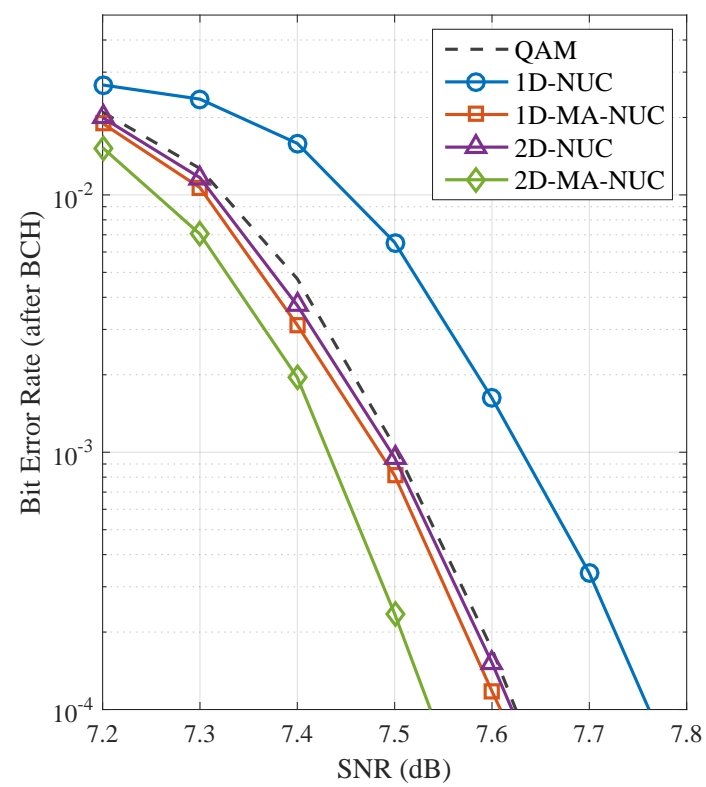

Figure 5.13: Performance comparison of QAM, 1D/2D-16NUC and 1D/2D-MA-16NUC. CR $4 / 15$ and $\mathrm{PI}=0 \mathrm{~dB}$, for i.i.d. Rice channel model.

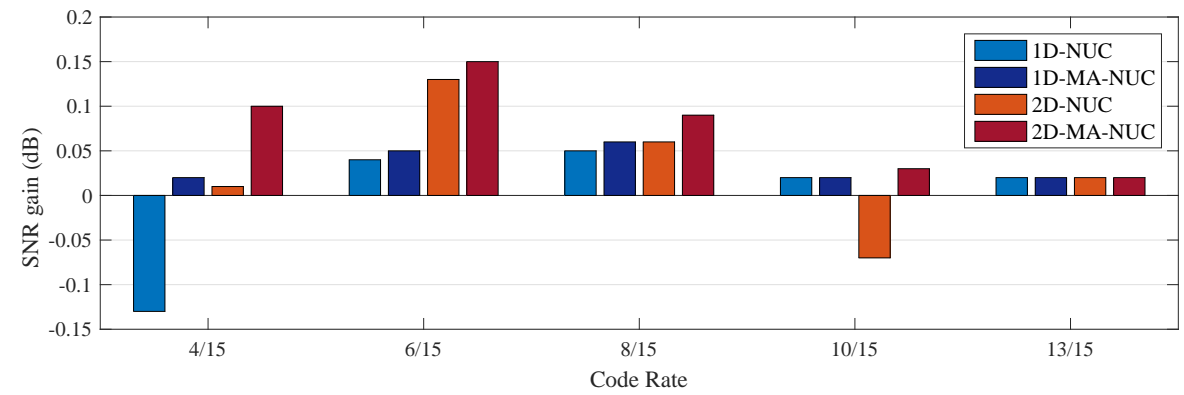

Figure 5.14: Performance gain (dB) of 1D/2D-16NUC and 1D/2D-MA-16NUC over QAM, for i.i.d. Rice channel model and five representative CRs, PI $=6 \mathrm{~dB}$.

achieving additional gains and avoiding performance loss for the use of a NUC not optimized for the SNR under study. As future work of this dissertation, MA-NUC optimization should be extrapolated to higher constellation orders, where larger performance gain are expected to be achieved. 


\subsection{Demapping Complexity Analysis}

As a generic analysis, in this section we consider 2D-NUCs with cardinality points $M$ of 16, 64, 256, 1024 and 4096 for a MIMO system, where 8, 12, 16, 20 and 24 bits per symbol are transmitted, respectively. Although 1k-2DNUC and $4 \mathrm{k}-2 \mathrm{DNUC}$ constellations are not considered in the rest of the thesis, the idea is to show the complexity implications when using 2D-NUCs and MIMO. As done in the rest of this chapter, it is assumed a number of transmit and receive antennas $N_{T}=N_{R}=2$. The complexity is defined as the number of distances or visited nodes to be computed in four different demapping schemes:

- Optimum ML

- ZF/MMSE equalization with optimum ML

- Max-log

- Sphere decoder with fixed complexity

Note that sphere decoders with variable complexity are out of the scope of this thesis. The optimum ML demapper needs to calculate all possible distances. Since it is assumed that only two transmit antennas $N_{T}$ are used, the complexity can be obtained as $M^{2}$. The same applies to max-log, since all distances need to be computed. ZF and MMSE linear equalizers transform the joint MIMO demapping process into independent single-antenna demapping blocks. In a $2 \times 2$ MIMO system, the ML demapper is transformed into two independent ML demappers with complexity $M$, so the number of distances calculated is only $2 M$. The complexity using ZF or MMSE equalizers is reduced to $75 \%, 87.5 \%, 96.8 \%, 99.2 \%, 99.8 \%$ and $99.9 \%$, for $8 \mathrm{NUC}, 16 \mathrm{NUC}, 64 \mathrm{NUC}$, 256NUC, $1024 \mathrm{NUC}$ and 4096NUC, respectively.

When using fixed sphere decoders such as SFSD, the complexity is drastically reduced as well. The complexity in this case is $M+\left(\log _{2} M\right)^{2}$. In a first step, the SD algorithm calculates the distances for a single antenna, i.e. $M$ nodes. Then, the SIC solution is applied to those nodes, reaching the $\left(\log _{2} M\right)$ sub-optimal hard solutions. The second term is related to the output LLRs calculated from those solutions [98]. Compared to ML or max-log, the reduction is extremely high. The complexity reduction of SFSD compared to ML or max$\log$ becomes especially effective for high orders of constellation. The complexity using ZF or MMSE equalizers is reduced to $73.4 \%, 87.5 \%, 97.5 \%, 99.5 \%, 99.8 \%$ and $99.9 \%$, for $8 \mathrm{NUC}, 16 \mathrm{NUC}, 64 \mathrm{NUC}, 256 \mathrm{NUC}, 1024 \mathrm{NUC}$ and $4096 \mathrm{NUC}$, respectively. The number of visited nodes using all considered demappers are shown in Table 5.1 and Fig. 5.15. 
CHAPTER 5. NON-UNIFORM CONSTELLATIONS FOR MIMO COMMUNICATIONS

\begin{tabular}{|c|c|c|c|}
\cline { 2 - 4 } \multicolumn{1}{c|}{} & ML / max-log & ZF / MMSE & SFSD \\
\hline Complexity & $M^{2}$ & $2 M$ & $M+\left(\log _{2} M\right)^{2}$ \\
\hline 16NUC & 256 & 32 & 32 \\
\hline 64NUC & 4096 & 128 & 100 \\
\hline 256NUC & 65536 & 512 & 320 \\
\hline 1kNUC & $1.04 \cdot 10^{6}$ & 2048 & 1124 \\
\hline 4kNUC & $1.67 \cdot 10^{7}$ & 8192 & 4240 \\
\hline
\end{tabular}

Table 5.1: Number of distances/nodes computed in a $2 \times 2$ MIMO system with 2D-NUCs.

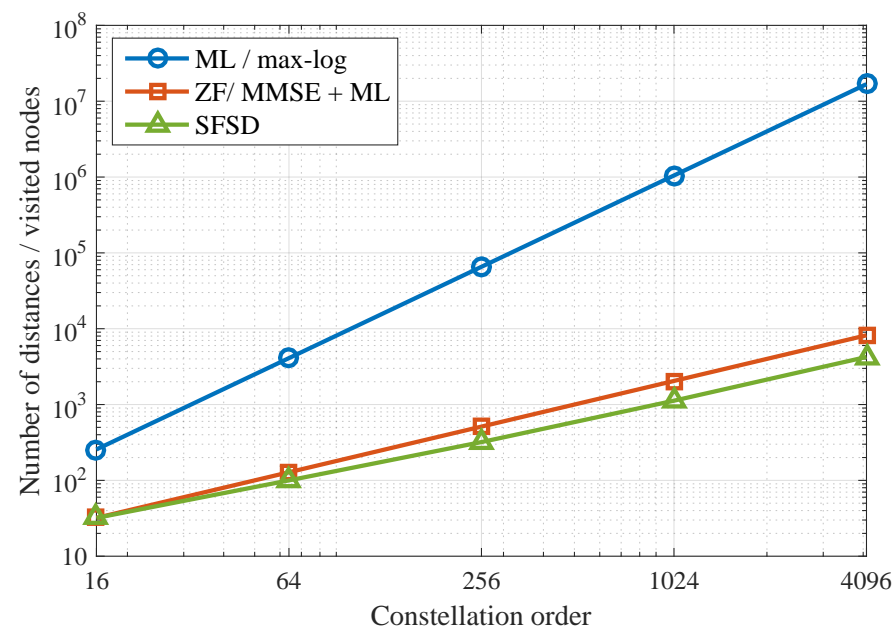

Figure 5.15: Number of distances or nodes visited with all considered demappers for 2DNUCs.

\subsection{Fixed Sphere Decoder for Two-Dimensional NUCs in MIMO}

Two different alternatives are considered in this thesis, i.e. linear equalizers and sphere decoders. The best option of the two methods depends on the constellation order considered. However, linear equalizers introduce a performance loss that becomes especially high at high CRs [82]. On the other hand, SD demappers achieve a sub-optimum max-log performance and provide a similar complexity reduction than linear equalizers, as shown in Fig. 5.15. Therefore, a good option to better approach the performance vs. demapping complexity trade-off is the use of sphere decoders with fixed complexity. 
In this thesis, we consider the particular case of SFSD. With SFSD, it is necessary to perform a quantization step called SIC that needs to be performed in separated I and Q components [80]. With 1D-NUCs, thanks to the squared shape, both components can be separated. However, this is not possible with 2D-NUCs. To facilitate the use of this demapper with 2D-NUCs, this section proposes an efficient pre-processing approach based on the Voronoi regions for the SIC quantization step [88]. The proposed technique is compared afterwards with the optimum ML and max-log demappers in terms of system performance. The proposed method obtains up to $97.5 \%$ of complexity reduction using 64NUCs, with a degradation lower than $0.1 \mathrm{~dB}$ and $0.7 \mathrm{~dB}$ compared to max-log and ML respectively. While results focus on 2D-NUCs for MIMO, the conclusions reached in this part of the thesis apply to all mobile communication systems, including unicast point-to-point ( $\mathrm{p}-\mathrm{t}-\mathrm{p})$ transmissions.

\subsubsection{Successive Interference Cancellation}

In MIMO systems, SIC is a particular subset of linear detectors [80]. SIC calculates the QR decomposition of the channel realization matrix $\mathbf{H}$ [109], where $\mathbf{Q}$ and $\mathbf{R}$ are an orthogonal and triangular matrix, respectively. It iteratively obtains an estimate of the received component from a single antenna. The estimated symbol will be used to obtain the components of the remaining antennas, as shown in Eq. 5.6.

$$
\hat{s}_{m}=\Gamma\left\{\frac{z_{m}-\sum_{i=m+1}^{N_{R}} R_{m, i} \hat{s}_{i}}{R_{m, m}}\right\}, m=N_{R}, \ldots, 1
$$

where $\hat{s}_{m}$ is the quantified estimated symbol from the $m^{\text {th }}$ antenna, $z_{m}$ is the received symbol multiplied by $\mathbf{Q}^{H}$, and $(\cdot)^{H}$ refers to the Hermitian operation. $\Gamma$ refers to the function that gives the quantized value from a continuous input. With QAM and 1D-NUCs, estimated complex symbols are separated into real and imaginary parts, and associated to the nearest value in each dimension. The only difference using QAM or 1D-NUCs is the use of a uniform or a nonuniform quantizer. However, using 2D-NUCs, the quantization is no longer direct, since constellations do not have a squared shape and I/Q components cannot be separated. A new method is required, in order to allow SIC to work with these new constellations, and is presented in the following section.

\subsubsection{Voronoi Regions Selection Algorithm}

In mathematics, a Voronoi diagram is a partitioning of a plane into regions based on distances to the points in a specific subset of the plane [110]. For 


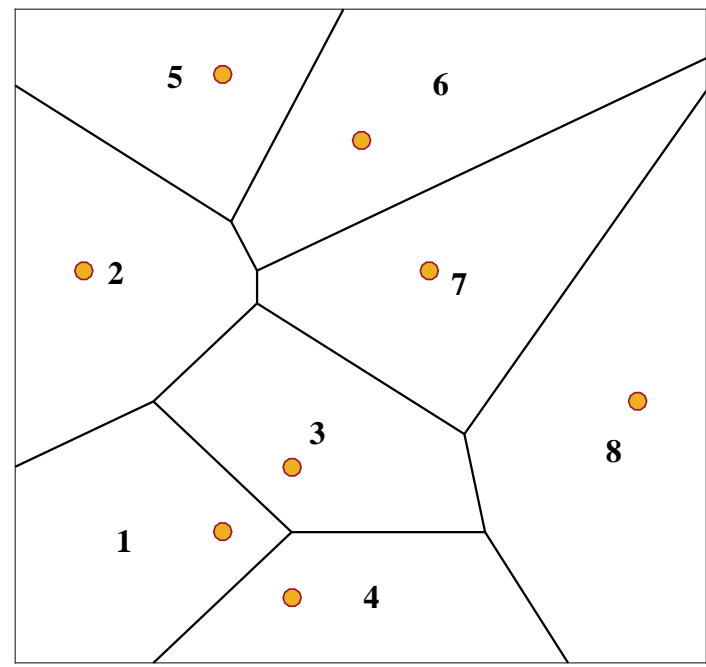

Figure 5.16: Voronoi regions calculated for 8 random points in a complex space, where each region takes an entire value associated to each point.

each constellation symbol, there is a corresponding Voronoi region in the constellation diagram consisting of the closest points to that symbol. Fig. 5.16 shows an example of the Voronoi regions calculated for 8 different positions generated randomly. Let $X$ be a metric space with distance function $d$. Let $I$ be a set of indexes and let $\left(P_{i}\right)_{i \in I}$ be an ordered collection of symbols in the space $X$. The Voronoi region $R_{i}$ associated to the symbol $P_{i}$ is the set of all points in $X$ whose distance to $P_{i}$ is lower than their distance to the other symbols $P_{j}$, where $j$ is any index different from $i$. This can be seen in Eq. 5.7. The Voronoi diagram is simply the tuple of cells $\left(R_{i}\right)_{i \in I}$.

$$
R_{i}=\left\{x \in X \mid d\left(x, P_{i}\right) \leq d\left(x, P_{j}\right), j \neq i\right\}
$$

The proposed method is based on this mathematical approach, and is called Voronoi Regions Selection (VRS). The algorithm consists of two different phases: the first phase calculates a Look-Up Table (LUT), from the Voronoi regions associated to a given NUC. This phase is only done once, before the communication takes place, and the LUT gets stored at the receiver. In the second phase, the receiver, which is already running, loads the LUT in order to receive the signal with VRS and SFSD demapping. In practice, the LUT is equivalent to an image, where each pixel has an integer value associated. The received symbol is assigned to a particular pixel, and the corresponding value 


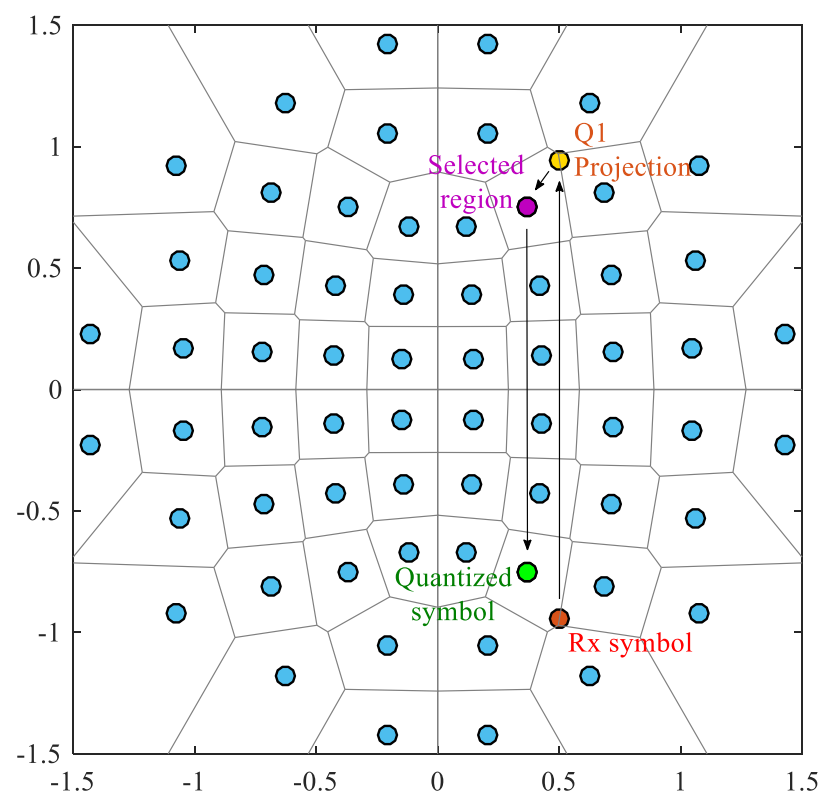

Figure 5.17: Example of Voronoi regions calculated for a 2D-64NUC, optimized for a SNR of $20 \mathrm{~dB}$.

is related to a constellation symbol, as shown in Fig. 5.17. Since constellations retain up-down and left-right symmetry, only the first quadrant needs to be stored. The other three quadrants can be derived by just changing the sign of real and imaginary parts of the received symbol. A LUT with squared shape has been selected as a compromise to accommodate different distributions of NUC constellation symbols, for practical implementations in real receivers. The LUT corresponding to every possible 2D-NUC used in a certain specification, e.g. ATSC 3.0, could be calculated beforehand and uploaded to the receiver memory.

The memory usage for a LUT depends on two different variables: the number of cells or pixels in one of the two I/Q axis considered $(k)$, which determines the total LUT size $(k \times k)$, and the number of bits $(b)$ assigned to each value/pixel of the LUT stored. The parameter $b$ is directly associated to the constellation size and only has memory implications. The maximum number of regions to store is a quadrant of the constellation, i.e. $M / 4$, being $M$ the number of symbols. Hence, the amount of bits can be calculated as $\left\lceil b=\log _{2}(M / 4)\right\rceil$. For instance, using a non-condensed $64 \mathrm{NUC}$ with $b=4$ and $k=40$, the stored LUT requires $6.40 \mathrm{Kbits}$. In case of condensation, the 


\section{CHAPTER 5. NON-UNIFORM CONSTELLATIONS FOR MIMO COMMUNICATIONS}
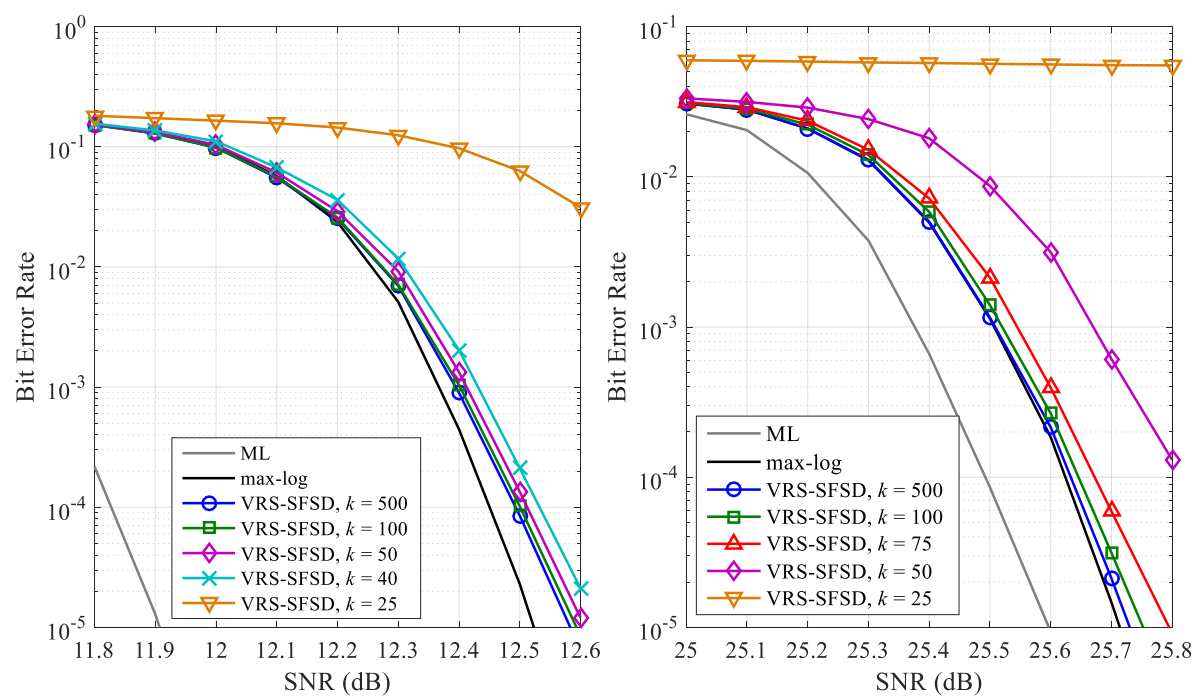

Figure 5.18: VRS with SFSD performance as a function of $k$, compared to ML and max-log. $64 N U C s$ are used in each antenna. CRs 5/15 (left) and 13/15 (right).

number of regions can be further reduced, as proposed in [104]. For example, using a $64 \mathrm{NUC}$ optimized for a $\mathrm{CR} 2 / 15$, the number of regions is reduced to $M=4$, so the number of bits required is only $b=2$.

To calculate the LUT, three steps are required. In the first step, the constellation symbols are grouped in clusters and removed, since they hinder the calculation of the Voronoi Regions. This step introduces an insignificant loss in performance, since lineal Euclidean distances among condensed symbols in normalized NUCs are almost negligible, lower than $10^{-2}$ [104]. In order to detect clusters, an array $L$ with polar coordinates (radius and angle) is defined, for each constellation symbol. The algorithm evaluates each element in $L$ individually. The difference is obtained for two threshold variables, $\rho_{T H}$ (radius) and $\alpha_{T H}$ (angle in radians). This step was already explained in Chapter 4, Section 4.2.2 (CSR algorithm). In the second step, the Voronoi Regions are calculated, and each region is numbered. The LUT is created and filled by assigning a particular binary value with $b$ bits to each Voronoi region. Finally, in a third step, a morphological filter is applied to the LUT in order to fill the possible gaps between regions.

In a following real transmission, the LUT previously calculated is loaded. For every received symbol, the SFSD demapper needs to perform the quantization step. Then, the received symbol is transformed to the first quadrant, and 
5.4 Fixed Sphere Decoder for Two-Dimensional NUCs in MIMO

\begin{tabular}{|c|cccc|}
\cline { 2 - 5 } \multicolumn{1}{c|}{} & $\mathbf{1 6 N U C}$ & $\mathbf{1 6 N U C}$ & $\mathbf{6 4 N U C}$ & $\mathbf{6 4 N U C}$ \\
\hline $\mathbf{5 / 1 5}$ & $\mathbf{1 3 / 1 5}$ & $\mathbf{5 / 1 5}$ & $\mathbf{1 3} / \mathbf{1 5}$ \\
\hline k & 18 & 25 & 40 & 75 \\
\hline Number of regions & 4 & 4 & 12 & 16 \\
\hline Memory (Kbits) & 0.65 & 1.25 & 6.40 & 22.50 \\
\hline
\end{tabular}

Table 5.2: Minimum $k$ and required memory for VRS

the corresponding Voronoi region is retrieved from the LUT, as Fig. 5.17 depicts. Finally, the associated closest symbol is obtained and the SFSD demapping process continues, using the whole constellation.

\subsubsection{Resolution vs. Performance Trade-Off}

As an example of application, this section provides some illustrative performance simulation results about the use of VRS with SFSD, compared to ML and max-log demappers. A LDPC code length of 64800 bits was used. Bit, time and frequency interleavers from ATSC 3.0 were employed [34]. We use 16NUCs and 64NUCs with CRs 5/15 and 13/15 in a spatial multiplexing MIMO 2x2 system, for an i.i.d. Rayleigh channel, where co-polar antennas are considered. The entries of the channel matrix are independent amongst each other, with zero-mean. An XPD of $6 \mathrm{~dB}$ is assumed, with independent time realizations between symbols [86]. Ideal channel estimation was used. For the VRS method, different values for parameter $k$ were evaluated. Note that the method VRS with SFSD and an infinite $k$ provides max-log performance.

Fig. 5.18 shows the performance of the proposed method for $64 \mathrm{NUC}$ with finite $k$ values. As Fig. 5.18 depicts, a minimum value for $k$ of 40 and 75 is necessary to obtain a performance loss under $0.1 \mathrm{~dB}$ compared to max-log. Two different factors affect the dependency on the CR. With low CRs, the higher error correction capability of the LDPC code allows to cope with a lower number of cells. In addition, the condensation of the NUC reduces the amount of regions to be considered in the VRS quantization of closest symbols. Note that, compared to ML, the proposed method introduces a performance loss of $0.7 \mathrm{~dB}$ for $\mathrm{CR} 5 / 15$ and $0.2 \mathrm{~dB}$ for CR13/15. Additional results for $16 \mathrm{NUC}$ are depicted in Table 5.2. The number of regions and memory requirements to store the LUT are provided as well. As Table 5.2 shows, both the number of LUT cells and memory required increase with constellation order and CR. 


\subsection{Conclusion}

In this chapter, a NUC re-optimization process is performed for $2 \times 2 \mathrm{MIMO}$ communications. The use of several antennas modifies both the channel model and the SNR range in which the constellations work. Additional features are introduced with this technology, such as the power imbalance (PI), cross-polar discrimination (XPD), or different demappers. The optimized constellations are called multi-antenna non-uniform constellations (MA-NUC). Without PI, we have shown that optimizing the same MA-NUC in both antennas provides similar results than optimizing separated constellations in the two antennas independently. It is better just to optimize a single constellation in this case, halving the degrees of freedom for optimization. Similar optimization parameters have been obtained for MMSE and ZF equalizers as well, while drastically reducing the demapping complexity. Different MIMO channel models have been analyzed, obtaining close results. In general, the re-optimization for MIMO has shown that, without PI between transmit antennas, constellations original designed for SISO are also a good alternative for MIMO. This assumption was already considered in the ATSC 3.0 standardization process.

MA-NUCs are effective only when exploiting a particular PI between antennas. Using a MA-16NUC, the highest performance gain has been obtained for a CR 4/15. The use of a different constellation per antenna that is adapted independently to each stream, and therefore to the power imbalance introduced, reduces in approximately $0.1 \mathrm{~dB}$ the minimum SNR required, compared to regular 2D-NUCs. This represents a promising result, which should be extended to higher-order constellations, where larger performance gain are expected to be achieved.

At the receiver, an efficient solution for 2D-NUCs and MIMO systems based on Voronoi regions has been proposed as well. In multi-antenna systems, the optimum ML demapping complexity grows exponentially with the number of antennas and the constellation order. To reduce it, suboptimum demappers can be used, such as SFSD. However, SFSD demappers do not work with 2DNUCs, since they perform a SIC quantization step that needs to be performed in separated I/Q components. The proposed method quantifies the closest symbol using Voronoi regions and allows SFSD demappers to work. Using VRS and SFSD and compared to ML and max-log demappers, the number of distances to be computed is reduced up to $87.5 \%$, and $97.5 \%$ for $16 \mathrm{NUC}$ and $64 \mathrm{NUC}$ respectively. The highest performance loss introduced is $0.1 \mathrm{~dB}$ compared to max-log and $0.7 \mathrm{~dB}$ compared to ML demappers. 


\section{Chapter 6}

\section{Conclusions and Future Work}

This dissertation has investigated and evaluated the use of non-uniform constellations (NUC) in digital terrestrial TV (DTT) systems that use bit-interleaved coded modulation (BICM). With uniform QAM constellations, the symbols are regularly spaced in the constellation diagram, i.e. with the same distance among symbols in both in-phase (I) and quadrature (Q) components. With NUCs, the gap between the BICM capacity of uniform QAM constellations and the theoretical Shannon limit is reduced, thanks to the shaping gain obtained from changing the positions of the different symbols in the I/Q space. Two different types of NUC have been investigated in this thesis. The first option is to keep the squared shape from QAM but relaxing the distribution between constellation symbols, with non-uniform distance between them. These constellations are called 1D-NUC and provide better signal-to-noise (SNR) performance results compared to QAM, without any complexity increase, since a 1D-demapper can be still used. The second option is to also relax the the square shape constraint, achieving a higher number of degrees of freedom (DoF) to optimize the symbol positions, thus achieving higher capacity gains. 2D-NUCs provide a better SNR performance than 1D-NUCs but with a higher demapping complexity, since a 2D-demapper is needed, i.e. I and $\mathrm{Q}$ components cannot be separated anymore. NUCs have been analyzed from both transmit and receive point of views, using either single-input single-output (SISO) or multiple-input multiple-output (MIMO) antenna configurations. This concluding chapter summarizes the main contributions of this thesis and suggests further topics of research beyond the results of this work. 


\subsection{Concluding Remarks}

\subsubsection{Non-Uniform Constellations Optimization}

Different orders of one- and two-dimensional non-uniform constellations have been designed. Constellation symbols are optimized so the average BICM capacity is maximized, using the Nedel-Mead simplex algorithm [89]. The algorithm iteratively looks for the optimum constellation symbols, modifying their positions in the I/Q plane. In this thesis, 1D-NUCs from 16 to 4096 symbols were designed, while 2D-NUCs were optimized up to 256 symbols. This assumption was also taken into account in the ATSC 3.0 standardization process [15]. The optimization of higher constellation orders is not feasible for the algorithm considered. The complexity of the optimization process is extremely high and the method does not find the optimum solution, reaching local minimums that do not provide the best result.

Table 6.1 shows the maximum BICM capacity gain obtained with 1D-NUCs and 2D-NUCs for each constellation order and i.i.d. Rayleigh channel. It also depicts the performance gain obtained in terms of reduced SNR when using these constellations and the optimum Maximum Likelihood (ML) demapper. Results show that 2D-NUCs always provide gains compared to $1 \mathrm{D}$. This additional gain comes from the more advanced optimization, which is worthwhile since the gains obtained are significative. Note that the use of two different channel models has been considered in a single optimization. Selecting different channel models affects the final constellation, with symbols located on the intermediate positions obtained in separated optimizations. Constellations for two transmission modes, i.e. fixed and mobile receptions, were also investigated. Since both modes require totally different SNR values, the combination is not optimum. It is better to focus on a particular SNR.

Another interesting concept is the use of condensed constellations. The idea is to transmit several symbols grouped in clusters, allowing to use more bits per symbol. In this case, the most significant bits provide similar robustness but the least significant bits are used to give additional information. On the other hand, the use of lower-order constellations allows to increase the CR, so in practice both constellations provide similar performance [34]. Another feature to take into account is power consumption. Although condensed, high-order constellations increase the power necessary to transmit all possible symbols. From this perspective, it is better to transmit low constellation orders that allow to use similar spectral efficiencies by using higher CRs.

2D-NUCs can be rotated to further increase the gain at high SNRs. The optimization process of Non-Uniform Rotated Constellations (NURC) has been described, first analyzing the performance in a single-RF transmission. Even 


\begin{tabular}{|c|c|c|c|c|c|c|}
\cline { 2 - 7 } \multicolumn{1}{c|}{} & \multirow{2}{*}{ Type } & \multicolumn{6}{|c|}{ Constellation symbols } \\
\cline { 3 - 7 } & & $\mathbf{1 6}$ & $\mathbf{6 4}$ & $\mathbf{2 5 6}$ & $\mathbf{1 0 2 4}$ & $\mathbf{4 0 9 6}$ \\
\hline BICM capacity & 1D & 0.04 & 0.07 & 0.18 & 0.32 & 0.43 \\
\cline { 2 - 7 } gain (bit/s/Hz) & 2D & 0.05 & 0.10 & 0.22 & - & - \\
\hline $\begin{array}{c}\text { Performance } \\
\text { gain (dB) }\end{array}$ & 1D & 0.05 & 0.32 & 0.65 & 1.05 & 1.20 \\
\cline { 2 - 7 } & 2D & 0.16 & 0.45 & 0.82 & - & - \\
\hline
\end{tabular}

Table 6.1: Maximum BICM capacity and performance gains per constellation order, for 1Dand 2D-NUCs, i.i.d. Rayleigh channel and optimum ML demapper.

though the demapping complexity is not increased, the SNR gain of NURCs in this case is not significant. The highest rotation gain is obtained for low-order constellations and high CRs, obtaining a maximum of $1.7 \mathrm{~dB}$ for i.i.d. Rayleigh channel using QPSK 13/15. This rotation gain comes from the fact that high CRs are not efficient for fading channels, where high-order constellations and low CRs provide better results. Note that rotation does not give any gain for AWGN channel. With multi-RF techniques such as Channel Bonding (CB) and Time-Frequency Slicing (TFS), the SNR gain is drastically increased, since I and $\mathrm{Q}$ components are transmitted in different RF channels. Without rotating NUCs, the higher the PI between RF channels, the larger the gain. The gain also depends on the $\mathrm{CR}$ and order of constellation. Regardless of the PI between RF channels, the highest gains are for very robust LDPC codes (low $\mathrm{CR}$ ) with a low-order modulation. When applying an additional rotation, the highest gain is achieved with the largest possible CR, when using the QPSK modulation. In this case, the SNR gain obtained is up to $6.7 \mathrm{~dB}$, with a PI of 9 $\mathrm{dB}$. When extending the results to $4 \mathrm{RF}$ channels, two additional scenarios have been considered: $3 / 4$ and $1 / 4 \mathrm{RF}$ channels with poor performance. The most important result derives from the second scenario, where the RF channel with poor performance obtains up to $8.7 \mathrm{~dB}$ of SNR gain using QPSK 13/15 (being $9 \mathrm{~dB}$ the maximum gain achievable). Additional results to these scenarios have been provided, when the RF channels present erasures. A 25\%, 50\% and $75 \%$ of erasures require CRs lower than $3 / 4,1 / 2$ and $1 / 4$ respectively in order to repair the loss of information. With an additional rotation, the maximum $\mathrm{CR}$ is increased to $13 / 15,9 / 15$ and $6 / 15$, respectively.

\subsubsection{Complexity Implications at the Receiver}

The use of NUCs on real receivers has different complexity implications. This thesis has investigated these constellations from two different points: demapping of constellation symbols and quantization of I/Q components or Log- 
Likelihood Ratios (LLR) obtained. Specific algorithms have been also proposed as alternative solutions to minimize the problems detected.

For the first case considered, it is known that the number of distances to calculate with QAM and 1D-NUCs can be drastically reduced by using a 1Ddemapper, as proposed in [26]. However, with 2D-NUCs and RCs, the demapper has to evaluate all distances in two dimensions. The algorithm proposed in this part is based on two different strategies. The first one is called Quadrant Search Reduction (QSR) and computes the LLRs discarding those distances whose probability of being computed is significantly low. It takes advantage of the symmetry that constellations provide, selecting a cluster of points to compute the LLRs. The second strategy is called Condensed Symbols Reduction (CSR), and exploits the condensation of NUCs, especially at low SNRs where some of the constellation symbols almost repeat the same position in the I/Q plane. It omits the computation of those distances which are similar to others already calculated, and replicates them. An example of application has been shown for 2D-256NUCs. Both strategies can be combined in order to reduce from $69 \%$ to $93 \%$ the number of required distances, depending on the CR, with almost no performance loss compared to the optimal ML demapper. The worst result is obtained for a high CR 13/15. The demapper can be extrapolated to the different orders of constellation considered in this thesis, i.e. $2 \mathrm{D}-16 \mathrm{NUC}$ and 2D-64NUC, but also to larger constellations such as $2 \mathrm{D}-1 \mathrm{kNUC}$ or $2 \mathrm{D}-4 \mathrm{kNUC}$.

Regarding the influence of NUCs on digital quantizers, it has been shown that quantization values for QAM and NUC are different. Not only constellation symbols are modified with these constellations, but also LLRs obtained after the demapping process. To reduce the amount of bits to store at the TDIL, a new strategy based on BICM capacity maximization via the NelderMead algorithm [89] has been proposed. It has been applied to both I/Q components and LLR values, selecting the option that provides better memory reduction percentages compared to conventional quantizers, for each modulation and CR. In general, non-uniform LLR quantization is the best choice in the sense of meeting the performance target with minimum memory requirements. The use of non-uniform quantizers adapted to the signal statistics provide slight improvements in terms of performance or alternatively in-chip memory savings.For the worst case considered, i.e. 256NUC with CR 13/15, a final reduction in TDIL memory of $30 \%$ compared to conventional quantizers has been achieved, when quantizing LLR values. Better results have been obtained for low-order constellations. With $16 \mathrm{NUCs}$, the total memory reduction ranges from $45.8 \%$ to $58.3 \%$. For $64 \mathrm{NUCs}$, reduction percentages from $47.2 \%$ to $58.3 \%$ have been also obtained. 


\subsubsection{Multi-Antenna Optimization and Complexity Re- duction}

A re-optimization process has been performed for $2 \times 2 \mathrm{MIMO}$ communications. The use of several antennas modifies both the channel model and the SNR range in which the constellations work. Additional features need to be considered, such as power imbalance (PI), cross-polar discrimination (XPD), or linear equalizers. The optimized constellations are called multi-antenna non-uniform constellations (MA-NUC). Without PI, optimizing the same MA-NUC in both antennas provides similar results than optimizing separated constellations in the two antennas independently. It is better just to optimize a single constellation, halving the degrees of freedom for optimization. Similar parameters have been obtained for MMSE and ZF equalizers as well, while drastically reducing the demapping complexity. Channel models specific for MIMO have been analyzed, obtaining close results. In general, the re-optimization for MIMO has shown that, without PI between transmit antennas, constellations original designed for SISO are also a good alternative for MIMO. This can be extended to ATSC 3.0, where SISO constellations are used in MIMO.

MA-NUCs are effective only when exploiting a particular PI between antennas. As an example of application, 16-MA-NUCs have been optimized. The highest performance gain has been obtained for a CR 4/15. The use of a different constellation per antenna that is adapted independently to each stream, and therefore to the PI introduced, reduces in approximately $0.1 \mathrm{~dB}$ the minimum SNR required, compared to regular 2D-NUCs. For the considered case, more condensed constellations are obtained for the second MIMO stream, which has lower signal power and therefore lower SNR for optimization. This represents a promising result, which should be extended to higher-order constellations, where larger performance gains are expected to be achieved.

At the receiver, an efficient solution for 2D-NUCs and MIMO systems based on Voronoi regions has been proposed as well. In multi-antenna systems, the optimum ML demapping complexity grows exponentially with the number of antennas and the constellation order. To reduce it, suboptimum demappers can be used, such as Soft Fixed-Complexity Sphere Decoders (SFSD). However, SFSD demappers do not work with 2D-NUCs, since they perform a successive interference cancellation (SIC) quantization step that needs to be performed in separated I/Q components. The proposed method quantifies the closest symbol using Voronoi regions and allows SFSD demappers to work. Using this method and compared to ML and max-log demappers, the number of distances to be computed is reduced up to $87.5 \%$, and $97.5 \%$ for $16 \mathrm{NUC}$ and $64 \mathrm{NUC}$ respectively. The highest performance loss introduced is $0.1 \mathrm{~dB}$ compared to max-log and $0.7 \mathrm{~dB}$ compared to ML demappers. 


\subsection{Constellation and CR Recommendation}

The conclusions reached in this dissertation help to understand the benefits and drawbacks of non-uniform constellations, both in transmission and reception, with or without being combined with other techniques such as rotation, $\mathrm{CB}$ or MIMO. This section is meant to give advice and recommendation about the optimum NUC and CR configuration to use for some representative use cases in broadcasting. The most important aspect of broadcast transmission is the spectral efficiency provided to guarantee a set of services. The spectral efficiency is related to several aspects such as the SNR, CB, or rotated constellations. Additional parameters need to be considered at the receiver, which represent a performance-complexity trade-off. In this analysis, the SNR is selected to guarantee a bit error rate (BER) lower than $10^{-4}$. The parameters to consider are listed below.

- Spectral efficiency. In this analysis, LDPC codes with additional Bose Chadhuri Hocquenghem (BCH) parity is considered. The spectral efficiency is calculated as:

$$
\gamma(\mathrm{bit} / \mathrm{s} / \mathrm{Hz})=\log _{2}(M) \cdot \frac{(L \cdot R)-B}{L}
$$

where $L$ is the LDPC code length, $M$ is the number of constellation symbols, $R$ is the coding rate, $B$ is the $\mathrm{BCH}$ parity rate.

- Rotation gain. Rotated constellations are used to improve the overall system performance at low constellation orders and high CRs. This feature is not critical upon selecting a modulation and CR combination, since the gain obtained comes from a poor SNR, due to the fading and the use of non-robust CR.

- Channel bonding gain:. The CB gain depends on three parameters: $\mathrm{CR}$, order of constellation and PI. This analysis assumes 2 RF channels. Regardless of the PI between RF channels and without applying any rotation to the constellation, the highest gains are for very robust LDPC codes with a low-order constellation.

- Demapping complexity. Number of distances to compute at the receiver. Parameter directly related with the constellation order and the type of constellation. With $1 \mathrm{D}-\mathrm{NUCs}$, distances are calculated in separated components. With $2 \mathrm{D}-\mathrm{NUCs}$, no separation is possible, and therefore the QCSR demapper proposed in Chapter 4 is used to efficiently reduce the number of distances to calculate. With MIMO, the solution proposed in Chapter 5 is used. 


\begin{tabular}{|c|c|}
\hline Service & Bit rate required (Mbps) \\
\hline SD & $0.8-1.8$ \\
\hline HD (1080p) & $2-3.5$ \\
\hline UHD (4k, HDR) & $15-20$ \\
\hline
\end{tabular}

Table 6.2: Picture quality of services and bit rate required. H.265 video coding standard is assumed [111].

- Implementation loss. Some inter-carrier interference can be caused by the phase noise values of receiver tuners, especially for high-order constellations. This parameter also comprehends differences between ideal and real receiver algorithms [28]. This analysis assumes no implementation loss for constellations from QPSK to 64NUC.

- Power consumption at the receiver. Parameter directly related to the constellation order. The use of high-order constellations increases the power necessary to demodulate all possible symbols. In this sense, the best option is to use low constellation orders that allow to use similar capacities, thanks to the use of higher CRs.

- Signal quantization at the receiver. The number of bits required in the TDIL are also considered as an additional feature to consider in the selection process. Overall, a lower number of bits is required when using low-order constellations with very robust CRs.

The correct selection of the parameters listed above is the key for transmitting a certain set of services the most efficient way and with the lowest complexity possible at the receiver. For a minimum SNR required, the optimum case is that which provides the best capacity but also entails low complex receive techniques, such as demapping or quantization. The selected mode must guarantee other quality parameters such as low implementation loss in real hardware receivers or low power consumption in transmission.

There is a direct relation between services required and the bit rate necessary to transmit them. According to the latest H.265 video coding standard [111], the bit rate requirements associated to a particular service are shown in Table 6.2. Notice that the bit rate is calculated as $C \times B$, where $C$ is the capacity and $B$ is the signal bandwidth in MHz. This analysis considers 6 $\mathrm{MHz}$ of signal bandwidth. Also note that the requirements for High Definition TV (UHDTV) depend on the use of complementary techniques such as High Dynamic Range (HDR) [112]. The transmission of a certain number of Standard Definition TV (SDTV), High Definition TV (HDTV) or UHDTV services depends on the particular scenario. 


\begin{tabular}{|c|c|c|}
\hline Use case & Fixed Rx & Mobile Rx \\
\hline Selected mode & $\begin{array}{c}64 \mathrm{NUC} \\
9 / 15\end{array}$ & $\begin{array}{c}16 \mathrm{NUC} \\
5 / 15\end{array}$ \\
\hline SNR (dB) & 14.5 & 4.3 \\
\hline Bit rate (Mbps) & 21.5 & 7.9 \\
\hline $\begin{array}{c}\text { Services } \\
\text { provided }\end{array}$ & $\begin{array}{c}6 \mathrm{HD} \\
1 \mathrm{UHD}\end{array}$ & $2 \mathrm{HD}$ \\
\hline $\begin{array}{c}\text { Demapping } \\
\text { complexity }\end{array}$ & 30 & 6 \\
\hline $\begin{array}{c}\text { Implementation } \\
\text { loss }\end{array}$ & \multicolumn{2}{|c|}{ No } \\
\hline $\begin{array}{c}\text { Power } \\
\text { consumption }\end{array}$ & 0 \\
\hline Rotation gain (dB) & \multicolumn{2}{|c|}{1.55} \\
\hline CB gain (dB) & 1.45 & 5.7 \\
\hline $\begin{array}{c}\text { Quantization } \\
\text { memory (Mbits) }\end{array}$ & 9.4 & \multicolumn{2}{|c}{} \\
\hline
\end{tabular}

Table 6.3: Modulation and CR selected for the use cases considered.

\section{Application to Use Cases}

Table 6.3 reveals an example of constellation and CR combinations recommended for transmission in several scenarios. For SISO communications, 1 UHD and 2 HD services have been assumed for rooftop fixed and portable mobile reception conditions, respectively. Channel models considered are i.i.d. Rice and DVB-NGH mobile. From Table 6.2, a single UHD service requires $20 \mathrm{Mbps}$, and $2 \mathrm{HD}$ services require $7 \mathrm{Mbps}$. In case of fixed reception, a 2D-64NUC with CR 7/15 has been selected, which provides a good capacitycomplexity trade-off for the bit rate required, and also requires a minimum SNR lower than $15 \mathrm{~dB}$, which is suitable for this type of reception. Moreover, no implementation loss is introduced, with very low power consumption. Although no rotation gain is achieved using this mode, it gives a SNR gain of $1.45 \mathrm{~dB}$ when $\mathrm{CB}$ with SNR averaging is used. On the other hand, the selected modulation for portable reception is a 2D-16NUC with CR 5/15. Both constellation order and $\mathrm{CR}$ have been modified in order to reduce complexity and SNR required at the expense of a lower capacity, and therefore, a smaller number of services. Note that Layered Division Multiplexing (LDM) is not considered in this analysis, which permits to use fixed and mobile services simultaneously with high spectral efficiency. 


\subsection{Future work}

\subsubsection{High-Order Two-Dimensional Non-Uniform Cons- tellations}

In this dissertation, constellations have been optimized up to 1D-4096NUC and 2D-256NUC orders. The algorithm utilized in this thesis does not reach an optimum solution for large numbers of DoF, thus making impossible to design higher-order constellations. The optimization method needs to be improved in order to design 2D-NUCs with 1024 and 4096 symbols, achieving extremely high capacity gains compared to QAM and 1D-NUCs, as shown in [24]. In turn, performance gains provided by these constellations should be thoroughly studied. The demappers proposed in this dissertation should be used with this constellations as well, in order to reduce the demapping complexity. Another option is to consider the condensation prior to optimization, for low CRs. In such case, only clusters of symbols need to be calculated, slightly reducing the capacity obtained but drastically reducing both the DoF and the number of iterations to reach the optimum solution.

\subsubsection{Further Optimization for MIMO Systems}

Chapter 5 has considered a re-optimization for $2 \times 2$ MIMO systems and constellations with 16 non-uniform symbols. Results have revealed that constellations optimized for SISO can be reused for MIMO if no power imbalance between transmit antennas is introduced. The power imbalance modifies the SNR target for optimization in each antenna. In this case, additional performance gains have been obtained. The study should be extended to high-orders of constellation such as 2D-64NUC or 2D-256NUC where the demapping complexity is still feasible, especially if the proposed SFSD demapper is used. The effect of the MIMO precoder in the optimized constellation has also to be investigated.

\subsubsection{The Future of Broadcasting: Constellations for 5G Communications}

The future of broadcasting is expected to evolve towards new scenarios where broadcast and cellular industries with different network infrastructures and business models are brought together to form a single converged network. This would enable an optimum use of the existing wireless infrastructure and spectrum [113]. Work is ongoing in the Third Generation Partnership Project (3GPP) to improve the efficiency of TV broadcast service delivery using fourth generation (4G) LTE networks. Thanks to LTE broadcast, the concepts used 
in recent standards such as optimized NUCs, as well as novel concepts could be considered. NUCs can provide important gains thanks to the uplink that provides additional information on channel conditions, thus adapting to the SNR and providing an optimum capacity.

NUCs should be also introduced in the fifth generation $(5 \mathrm{G})$ of mobile communications for broadcasting purposes. The introduction of $5 \mathrm{G}$ broadcast also requires to study the most promising candidate $5 \mathrm{G}$ new modulation techniques, such as Frequency and Quadrature Amplitude Modulation (FQAM) or Offset Quadrature Amplitude Modulation (OQAM), in terms of increased capacity and performance. FQAM is a combination of Frequency Shift Keying (FSK) and QAM [114]. From the transmitter point of view, the use of FQAM should be further investigated and combined with NUCs. The use of this new proposal would not require a simple ML demapper in order to calculate the LLR values. In this sense, a new demapper that computes the LLRs for both the constellation and the frequency component needs to be studied. Regarding OQAM, it performs a time shift of half the inverse of the sub-channel spacing between the real and imaginary parts of a complex symbol. This constellation is combined with Filter Bank Multi-Carrier (FBMC) [115] to reduce the out-of-band interference levels and avoid the use of a guard interval (cyclic prefix) which represents an overhead for OFDM. The use of non-uniform distances between real and imaginary parts should be carefully studied in this new context. Other innovative $5 \mathrm{G}$ air-interface technologies to be considered that may affect the design process of these constellations are: massive MIMO [116], i.e. the use of a large number of transmit antenna elements to perform narrow two-dimensional beamforming; and Non-Orthogonal Multiple Access (NOMA), which is equivalent to LDM and allows simultaneously to use the same frequency resources for more than one transmission [117]. 


\section{Appendix A}

\section{Physical Layer Simulator}

The results presented in this dissertation have been obtained making use of a Physical Layer (PHY) simulator that assesses the performance and capacity gains of non-uniform constellations, among other features. This appendix presents the simulator in detail, which is based on the ATSC 3.0 specification and adapted for the particular studies carried out in this thesis. A similar architecture has been taken for the implementation of $2 \times 2$ MIMO transmissions. This simulator was implemented and used during the standardization process of the ATSC 3.0 specification [15]. The different blocks that define the complete simulator were validated with other participants in this process. The simulator is formed by five main components: parameters initialization, transmitter, channel models, receiver and error measurement, as shown in Fig. A.1. From the initial configuration introduced to the simulator, the parameters necessary to use in the simulator are pre-loaded. This includes from basic parameters such as the modulation, CR, FFT size or SNR; to more complex configurations such as interleaving indexes or LDPC matrixes. Once parameters are loaded, the core simulator starts, and no additional configuration is needed. The core simulator is formed by the transmitter, channel and receiver. At the end of the simulations, two possible options for error measurement are available: SNR performance in terms of bit and frame error rate, or mutual information calculation. The common transmit to receive diagram as well as channel models utilized for SISO and MIMO are presented in following sections.

\section{A.1 Transmitter Block Diagram}

Fig. A.2 depicts the generic transmitter used in this dissertation for simulation purposes. Blocks in black show the generic transmit SISO simulator, which is 


\section{APPENDIX A. PHYSICAL LAYER SIMULATOR}

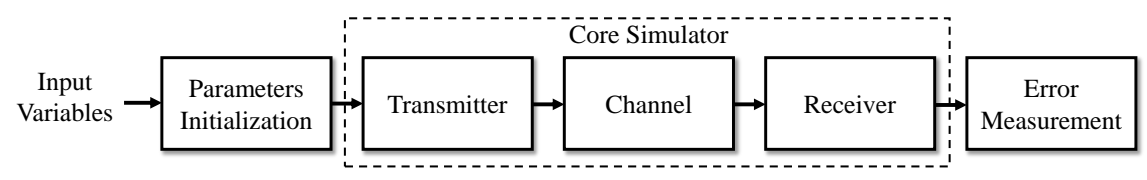

Figure A.1: Generic PHY simulator block diagram.

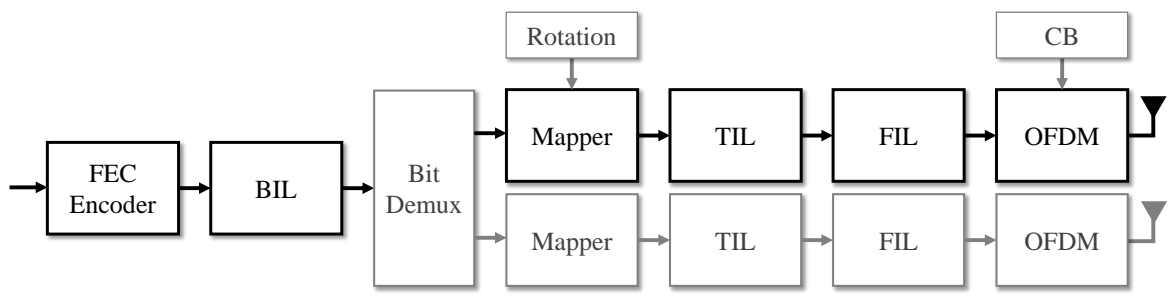

Figure A.2: Generic transmitter block diagram for SISO and MIMO used in the PHY simulator.

formed by the concatenation of FEC coding, bit interleaving, mapping, time and frequency interleaving, and waveform generation. Blocks in grey represent optional technologies additionally implemented. For MIMO, encoding and bit interleaving are jointly performed for both streams, while mappers, frequency and time interleavers, and waveform generators are applied separately. In addition, a bit demultiplexer is needed before demapping. The simulator also allows to rotate constellations, or to divide the data into two different channels with channel bonding, by simply activating or deactivating some additional parameters.

\section{Forward Error Correction (FEC)}

The PHY simulator implements forward error correction based on a concatenation of the baseband packet payload (data bits) with size $K_{P A Y}$, an outer code (BCH) that introduces $M_{O U T}$ additional parity bits, and a inner code (LDPC) with $M_{I N}$ parity bits. In total, the output FEC frame has size $L$ bits, as Fig. A.3 shows. The simulator employs LDPC inner codes from ATSC 3.0 [35]. For SISO, there are two different LDPC code lengths defined, i.e. $L=16200$ bits (short codes) and $L=64800$ bits (long codes). For MIMO, only the long code is specified. ATSC 3.0 permits to use a set of 12 coding rates from 2/15 to 13/15, with step $1 / 15$. Therefore, there is a total amount of 24 different LDPC configurations to select. Note that, although ATSC 3.0 implements three options for the outer code, in this dissertation we only consider the use of $\mathrm{BCH}$. The 


\section{A.1 Transmitter Block Diagram}

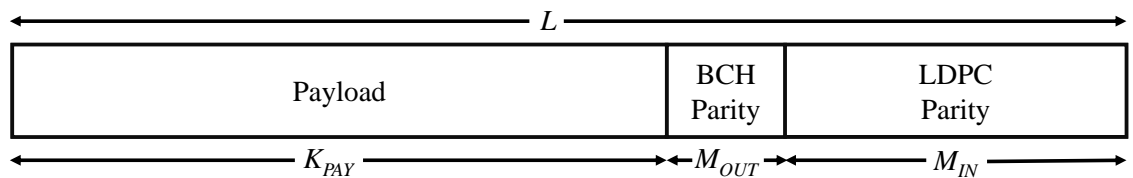

Figure A.3: Format of simulator FEC frames with BCH and LDPC coding.

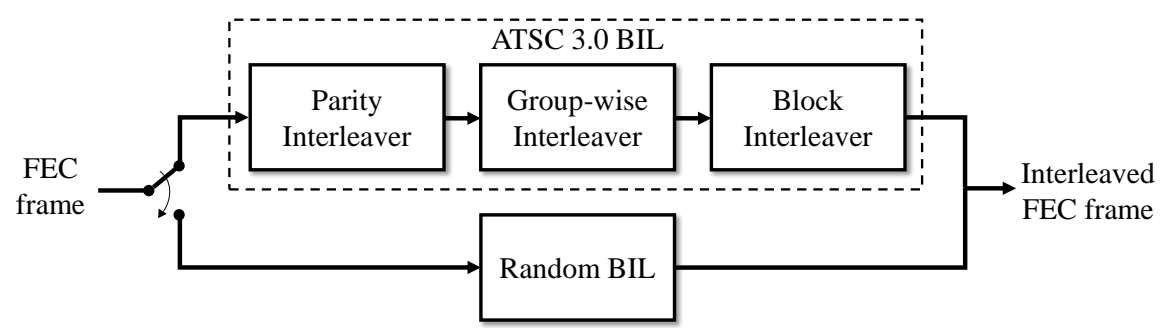

Figure A.4: Format of simulator bit interleaving. Two possible options: ATSC 3.0 or random BIL.

outer BCH code is used to lower the inherent LDPC error floor by correcting a predefined number of $M_{O U T}=12$ bit errors .

\section{Bit Interleaver (BIL)}

Two different options are considered in the simulator for BIL, which are a random BIL and the BILs from ATSC 3.0. In both cases, the BIL block takes a FEC frame with size $L$ as input. The output is just a bit interleaved FEC frame whose length does not change after the operation. On one hand, BILs from ATSC 3.0 consist of a parity interleaver followed by a group-wise interleaver and a block interleaver, see Fig. A.4. Bit interleaving permutations are designed so the performance with NUCs is maximized. Note that MIMO reuses the BILs from the SISO baseline and are not specifically optimized for multi-antenna transmissions. Logically, the use of these BILs with different constellations such as QAM may introduce an additional loss, since they are not designed for such case. When comparing different types of constellations, it is better to use a random BIL.

\section{Bit Demultiplexer}

In case of $2 \times 2 \mathrm{MIMO}$ transmissions, the bit demultiplexer distributes the output bits from the BIL into the two separate mappers, one per transmit antenna. With symmetric configurations, where the same order of constellation 


\section{APPENDIX A. PHYSICAL LAYER SIMULATOR}

is used in both antennas, the $L$ bits are divided into two groups of $L / 2$ bits. Odd bits are mapped to the first transmit antenna, while even bits are mapped to the second transmit antenna. With SISO, the bit demultiplexer block is not applied.

\section{Mapper}

After dividing the data into two streams (in case MIMO is selected), FEC encoded and bit interleaved bits are mapped to complex-valued constellation symbols. The input to the constellation mapping block in each stream is a FEC frame and the output is a FEC block. In addition to QPSK, the simulator implements 1D-NUCs for cardinalities from 16 to 4096 points, and 2D-NUCs for cardinalities of 16, 64 and 256 points. It also permits to use uniform QAM constellations up to 4096 symbols. The MIMO scheme allows to use either the SISO antenna baseline constellations, or those re-optimized for multi-antenna communications, called in this dissertation 1D- and 2D-MA-NUC. For the particular case of 6 and 10 bits per symbol, it also allows to use either asymmetric configurations or $8 \mathrm{NUC}$ and $32 \mathrm{NUC}$ constellations in each antenna. Constellation rotation is additionally introduced as an optional feature. The use of rotated NUCs is described in detail in Chapter 3, Section 3.3. In that section, rotation angles are also shown.

\section{Time Interleaver (TIL)}

In ATSC 3.0, time interleaving is processed in two different ways depending on the number of PLPs in which the data is transmitted. When using a single PLP mode, a convolutional TIL is used, whereas for multiple PLPs a hybrid TIL composed of cell interleaver, twisted block interleaver, and a convolutional delay-line is employed [118]. Since the use of multiple PLPs is out of the scope of this dissertation, the simulator only implements the convolutional TIL for a single PLP. The input to the convolutional TIL is a sequence of symbols coming from the mapper output. The TIL consists of $N_{\text {row }}$ delay lines, with the $k$ th line having $k$ delay elements, $k=0,1, \ldots, N_{\text {row-1 }}$, and each delay element capable of storing one symbol. The number of columns $N_{\text {col }}$ is just $N_{\text {row }}-1$. Hence, the different inputs and outputs are controlled by two commutators, as shown in Fig. A.5, cyclically switching downwards after one cell is written in or read out, respectively.

When the interleaver is at position $k$, a new symbol is written to this delay line. As a first step, each delay content from this line is shifted to the immediate right position, and the content from the right-most position is output through the output commutator. In a second step, the input symbol is written to the left-most delay element of the used line. Finally, both commutators are moved 


\section{A.1 Transmitter Block Diagram}

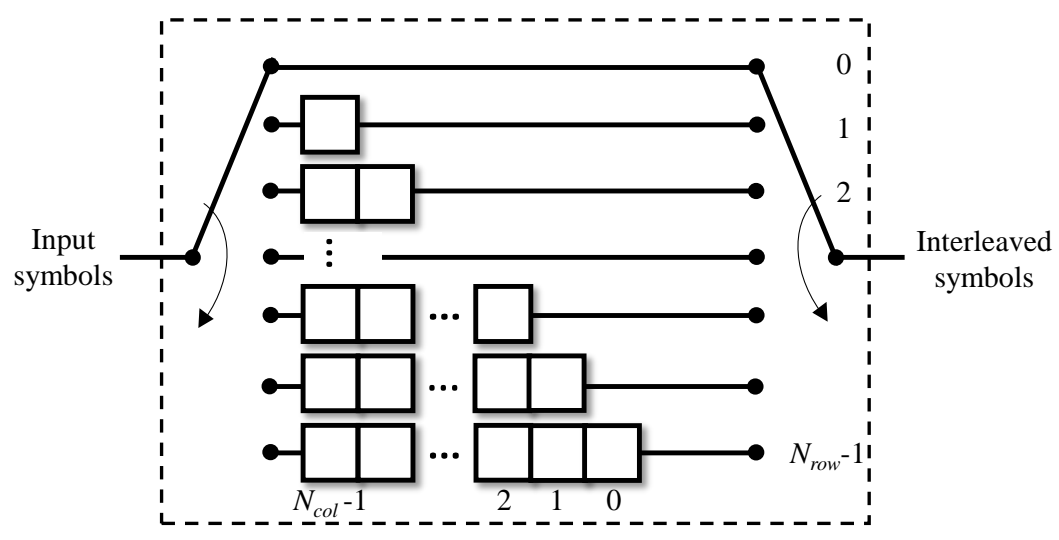

Figure A.5: Convolutional time interleaving employed in the simulator.

to the next line $k+1$. The process is cyclically repeated for $N_{\text {row }}$. Possible values in the simulator for $N_{\text {row }}$ are 1024, 887, 724 and 512, which represent a time interleaving depth of $200 \mathrm{~ms}, 150 \mathrm{~ms}, 100 \mathrm{~ms}$ and $50 \mathrm{~ms}$, respectively. Note that for MIMO scheme, both signals use the same time interleaver depth.

\section{Frequency Interleaver (FIL)}

The frequency interleaver takes data symbols coming from the TIL of one OFDM symbol, and separates error bursts occurring in the frequency domain making use of three address generators. The different address generators implemented in the simulator are also specified in ATSC 3.0, one per each FFT size available: $8 \mathrm{~K}, 16 \mathrm{~K}$ or $32 \mathrm{~K}$. Each address generator consists of three blocks: the toggle block, a basic interleaving sequence generator with a wire permutation, and a symbol offset generator. The main idea behind this FIL is to cyclically shift the value resulting from the combination of the output values of the three generation blocks. An additional address check block is used to validate whether the generated address is within range of the allowable addresses for the particular OFDM symbol being frequency interleaved or not. For more information, readers can refer to [119].

\section{Waveform Generation}

The waveform generator consists of a pilot insertion (in case real estimation is selected), followed by an inverse FFT and the GI insertion. Since broadcast transmissions do not use return channel, it is necessary to transmit additional 


\section{APPENDIX A. PHYSICAL LAYER SIMULATOR}

information about the channel. Several cells within the OFDM frame are modulated at a boosted power level with this information, whose value is known to the receiver. Different pilots such as scattered, continual, or edge pilots are transmitted. Information about the pilot patterns used for scattered pilots can be found in [15]. Note that no pilot insertion is needed for ideal estimation. The resulting OFDM signal is then converted into the time domain. For the resulting time-aligned frame, a guard interval is inserted to ensure that distinct transmissions with same information do not interfere with one another, such as in SFN environments. GIs from ATSC 3.0 are used in the simulator. In case channel bonding is used, the output of 2,3 or $4 \mathrm{RF}$ channels is combined to achieve greater data rates than can be achieved in one channel. The use of CB is introduced as an additional feature in this simulator, and it is explained in Chapter 3, Section 3.4.

\section{A.2 Receiver Block Diagram}

The transmitted signal is then passed through a specific channel model previously defined in the parameters initialization and taken as an input to the receiver. Fig. A.6 depicts the generic receiver used in this thesis. As done for the transmitter, blocks in black show the generic transmit SISO simulator, which is formed by the concatenation of a waveform detector (with or without using channel bonding), frequency and time de-interleaving, demapping, bit de-interleaving, and FEC decoding. Blocks in grey represent optional technologies additionally implemented. For MIMO, mappers, frequency and time de-interleavers, and waveform detection are applied separately, while decoding and bit de-interleaving are jointly performed for both streams. A MIMO demapper is needed in this case, which outputs a single bit vector from the two received complex-valued signals. The simulator also allows to perform quantization at the time de-interleaver.

From the received signal, the GI is extracted and the signal is transformed into the frequency domain. In case real estimation is used, pilots from the output signal are also extracted, and the data is stored in a new complex vector (or vectors). Frequency and time de-interleavers are then performed just using the FIL and TIL indexes stored and applied in transmission. The demapping converts the received data symbols into bits and bit de-interleaving is applied, also using the stored indexes. Finally, parity bits are extracted and the resulting bits are compared to transmitted bits in order to obtain bit and frame error rates for a particular SNR. Since most of receiver parts just need to undo the process used in transmission, only features related to this dissertation are detailed in following sections: demapping and quantization. 


\section{A.2 Receiver Block Diagram}

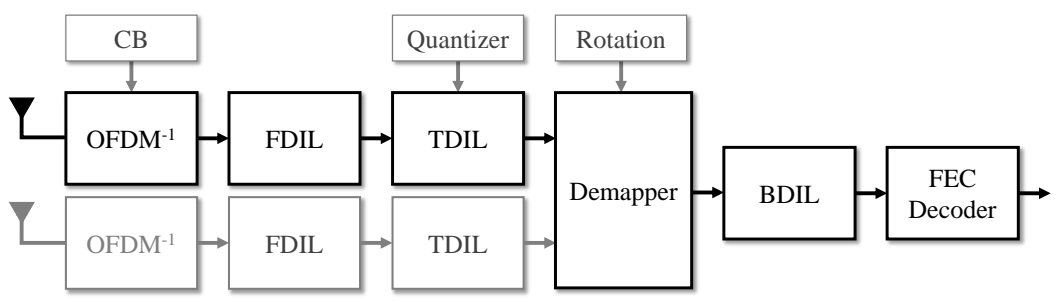

Figure A.6: Generic receiver block diagram for SISO and MIMO used in the PHY simulator.

\section{Demapper}

The simulator implements different soft-demappers that can be used in order to convert the received symbols into log-likelihood ratios. Eligible demappers depend on the number of transmit and receive antennas. The possible options considered in this simulator are the following:

- SISO:

- Maximum-Likelihood (ML)

- Max-log

- Quadrant Search Reduction (QSR)

- Condensed Symbols Reduction (CSR)

- Quadrant Condensed Search Reduction (QCSR)

- MIMO:

- Maximum-Likelihood (ML)

- Max-log

- Soft Fixed-Complexity Sphere Decoder (SFSD)

- Soft Fixed-Complexity Sphere Decoder (SFSD) based on Voronoi regions

In addition, ML and max-log MIMO demappers can be efficiently combined with ZF and MMSE equalizers, which reduce the demapping complexity and permit the use of two independent SISO demappers.

\section{Quantizers}

Quantization is an optional operation implemented for SISO receivers. Depending on the configuration selected, quantization is performed at the TDIL before 


\section{APPENDIX A. PHYSICAL LAYER SIMULATOR}

or after the demapping takes place, i.e. demapping and TDIL blocks are accordingly reordered. Conventional receivers usually perform TDIL immediately before the demapping process takes place. In such case, constellation symbols and channel estimates are stored in order to perform time de-interleaving. In case both operations are swapped, quantization is performed in a different point of the receiver chain, i.e. output LLRs at the demapper are quantized.

\section{A.3 Channel Models}

In this section, the different channel models used throughout the dissertation are explained, for $2 \times 2 \mathrm{MIMO}$ configurations with cross-polar antennas. Note that channels described can be easily extrapolated to SISO transmissions by just simplifying the models provided, considering one antenna in both transmitter and receiver. For the considered system, the discrete received signal is modeled as in Eq. A.1.

$$
\mathbf{y}=\mathbf{H s}+\mathbf{w}
$$

where $\mathbf{s}$ is a $2 \times 1$ transmitted vector, $\mathbf{y}$ is a $2 \times 1$ received vector, $\mathbf{H}$ is a generic channel matrix with correlated fading and cross-polar antennas, and $\mathbf{w}$ is the $2 \times 1$ additive circularly symmetric complex Gaussian noise. The matrix $\mathbf{H}$ considers that one transmit/receive antenna has vertical polarization and the other transmit/receive antenna has horizontal polarization, or vice-versa. The matrix $\mathbf{H}$ can be multiplied in turn by two additional parameters, i.e. the power imbalance between the two transmit antennas, and cross-polar discrimination (XPD) factor. The use of these parameters is explained in Chapter 2, Section 2.1.1. Note that channel models based on measurement campaigns such as the DVB-NGH mobile and MGM channels already include the XPD factor into their definitions.

\section{AWGN Channel}

The Additive White Gaussian Noise (AWGN) channel only introduces an additive circularly symmetric complex Gaussian noise where $\sigma^{2}$ is the noise power. The channel matrix $\mathbf{H}$ is a rectangular $2 \times 2$ matrix where all elements have value 1:

$$
\mathbf{H}=\left[\begin{array}{ll}
1 & 1 \\
1 & 1
\end{array}\right]
$$


For SISO, the channel is simply $h=1$. This channel, although simple, represents an accurate model for a wide set of communication channels, and can be combined with the additional channel features described above.

\section{i.i.d. Rayleigh}

When a MIMO system has sufficiently separated and equally polarized antennas with many scatters, the elements of the matrix $\mathbf{H}=\hat{\mathbf{H}}$ can be modeled using an ideal identically distributed (i.i.d.) complex Gaussian channel with zero-mean. For this channel, each matrix component can be modeled independently using the following expression:

$$
\hat{h}_{i j} \sim \mathcal{C N}\left(0, \frac{1}{2}\right), i=1,2, j=1,2
$$

Fading components with those characteristics are usually known as i.i.d. Rayleigh distributed. In this dissertation, we consider that the separation between antennas is wide enough, and/or scattered components do not come from the same direction. In this case, it is said that channel components are uncorrelated [120]. Low fading correlation with cross-polarized antennas relays on the fact that the fading experimented by each polarization path possesses a low degree of correlation [27]. This channel model $\hat{\mathbf{H}}$ represents reliably scenarios where there is NLoS between the transmitter and the receiver, such as portable indoor reception scenarios.

\section{i.i.d. Ricean}

The i.i.d. Rayleigh channel can be further extended to also consider the scenario where there is LoS. This channel is called i.i.d. Ricean channel. The LoS component can be modeled using Eq. A.4.

$$
\overline{\mathbf{H}}=\left[\begin{array}{ll}
\exp \left(j \theta_{11}\right) & \exp \left(j \theta_{12}\right) \\
\exp \left(j \theta_{21}\right) & \exp \left(j \theta_{22}\right)
\end{array}\right]
$$

where $\theta_{i j}$ is a fixed random phase uniformly distributed between $[0,2 \pi)$. The channel matrix values $\mathbf{H}$ are modified for the i.i.d Ricean channel calculation:

$$
\mathbf{H}=\sqrt{\frac{K}{1+K}} \overline{\mathbf{H}}+\sqrt{\frac{1}{1+K}} \hat{\mathbf{H}}
$$

where $\overline{\mathbf{H}}$ represents the LoS component, $\hat{\mathbf{H}}$ is the NLoS component, and $K$ models the power ratio between both components. A value $K=10$ has been used in this dissertation since it is a suitable value to represent a channel model 


\section{APPENDIX A. PHYSICAL LAYER SIMULATOR}

under rooftop fixed reception conditions [28]. Note that, if $K=0$ is selected, then $\mathbf{H}=\hat{\mathbf{H}}$ and the i.i.d. Rayleigh channel is applied.

\section{DVB-NGH Mobile Channel}

In the DVB-NGH standardization process, three different channels were used in order to evaluate the performance of the MIMO system proposed in a realistic scenario with mobile or portable reception. These channels were extracted from a measurement campaign carried in Helsinki in 2010. The specific channels permit to model three different scenarios, i.e. outdoor mobile, outdoor portable and indoor portable reception. In this dissertation, only mobile reception with high speed is considered. For additional information on the other two channels defined, readers can refer to [121]. The time domain channel model for the considered MIMO configuration is expressed in Eq. A.6.

$$
\mathbf{H}(t, \tau)=\left[\begin{array}{ll}
h_{11}(t, \tau) & h_{12}(t, \tau) \\
h_{21}(t, \tau) & h_{22}(t, \tau)
\end{array}\right]
$$

where $h_{i j}(t, \tau)$ is the impulse response between the $j$ th transmit antenna and the $i$ th receive antenna, $t$ is the time domain, and $\tau$ is the delay. The channel model can be decomposed into LoS and NLoS components, and expressed in vector form as in Eq. A.7.

$$
v e c\left(\mathbf{H}^{T}(t, \tau)\right)=\overline{\mathbf{h}} \delta(t)+\sum_{n=1}^{8} \sqrt{\mathbf{R}_{n}} \hat{\mathbf{h}}_{w, n} \delta\left(t-\tau_{n}\right)
$$

where $\overline{\mathbf{h}}$ is the LoS term, and the summation over the eight taps $\hat{\mathbf{h}}$ represents the NLoS term. For the $n$th tap, the covariance matrix $\mathbf{R}_{n}$ describes the correlation between the channel paths, and $\hat{\mathbf{h}}_{w, n}$ is the i.i.d complex Gaussian random vector of size $2 \times 1$.

DVB-NGH models are based on a power delay profile of 8 taps, with high cross-polarization discrimination $X$, and a concrete $K$ factor. In particular, the DVB-NGH mobile channel assumes $X=4$ and $K=\infty$ for the first tap, i.e. only LoS component is considered. The power delay profile for the DVB-NGH mobile channel is presented in Table A.1. It includes for each tap an excess delay, and co-polar/cross-polar power gains.

Note that the cross-polar gain $\sigma_{12}^{2}\left(\tau_{n}\right)=\sigma_{21}^{2}\left(\tau_{n}\right)$ can be easily calculated from the co-polar gain and the $X$ factor as $\sigma_{11}^{2}\left(\tau_{n}\right)-6(\mathrm{~dB})$. The spatial correlation matrix for taps $n=2, \ldots, 8$ is presented below. 


\begin{tabular}{|c|c|c|}
\hline Tap number, $n$ & Excess delay, $\tau_{n}(\mu \mathrm{s})$ & $\begin{array}{c}\text { Co-polar gain } \\
\sigma_{11}^{2}\left(\tau_{n}\right)=\sigma_{22}^{2}\left(\tau_{n}\right)(\mathrm{dB})\end{array}$ \\
\hline $1(\mathrm{LoS})$ & 0 & -4 \\
\hline 2 & 0.1094 & -7.5 \\
\hline 3 & 0.2188 & -9.5 \\
\hline 4 & 0.6094 & -11 \\
\hline 5 & 1.109 & -15 \\
\hline 6 & 2.109 & -26 \\
\hline 7 & 4.109 & -30 \\
\hline 8 & 8.109 & -30 \\
\hline
\end{tabular}

Table A.1: Power delay profile of the DVB-NGH mobile channel [121].

$$
\mathbf{R}_{n}=\sigma_{11}^{2}\left(\tau_{n}\right)\left[\begin{array}{cccc}
1 & 0.06 & 0.06 & 0.05 \\
0.06 & 0.25 & 0.03 & 0.05 \\
0.06 & 0.03 & 0.25 & 0.06 \\
0.05 & 0.05 & 0.06 & 1
\end{array}\right]
$$

For the first tap, the energy is pure LoS, so there is no Rayleigh part to the fading. Hence, $\mathbf{R}_{1}=[0]_{4 x 4}$. In mobile channels, the time dependent variation of the channel realizations produce frequency shifts, and therefore the Doppler effect introduced has to be taken into account. The maximum frequency shift $f_{D}$ produced at the receiver is modeled with:

$$
f_{D}=\frac{v}{c} f_{c} \cos (\alpha)
$$

where $v$ is the receiver speed, $c$ is the speed of light, $f_{c}$ is the RF frequency employed, and $\alpha$ is the angle between directions of receiver and signal. In [121], it was observed that the experimental methodology of pushing a trolley at approximately constant speed was not sufficiently accurate to produce quantitative results. Therefore, it was decided to use power spectral density also known as Jakes-Doppler spectrum to better model the Rayleigh fading taps obtained. The Jakes-Doppler spectrum is specified to be used at a particular tap in conjunction with a fixed frequency offset proportional to $f_{d}$. The spectra of each tap is shown below:

$$
\begin{cases}S\left(f-3 f_{D} / 4, f_{D} / 4\right) & p=2,3 \\ S\left(f+3 f_{D} / 4, f_{D} / 4\right) & p=4,5,6,7,8\end{cases}
$$

where the tap $p=1$ is LoS, with no Doppler shift. 
APPENDIX A. PHYSICAL LAYER SIMULATOR

\begin{tabular}{|c|c|c|c|}
\hline Tap number, $n$ & Excess delay $\tau_{n}(\mu \mathrm{s})$ & $\begin{array}{c}\text { Co-polar gain } \\
\sigma_{11}^{2}\left(\tau_{n}\right)=\sigma_{22}^{2}\left(\tau_{n}\right)(\mathrm{dB})\end{array}$ & $X_{n}$ \\
\hline $1(\mathrm{LoS})$ & 0 & -0.943 & 0.0303 \\
\hline $1(\mathrm{NLoS})$ & 0 & -8.11 & 0.0572 \\
\hline 2 & 0.2632 & -22.97 & 0.118 \\
\hline 3 & 0.5264 & -32.27 & 0.208 \\
\hline 4 & 0.7896 & -35.5 & 0.257 \\
\hline 5 & 1.448 & -37 & 0.272 \\
\hline 6 & 2.501 & -38.5 & 0.277 \\
\hline 7 & 3.554 & -40.88 & 0.288 \\
\hline 8 & 4.607 & -43.94 & 0.313 \\
\hline
\end{tabular}

Table A.2: Power delay profile of the MGM rooftop fixed channel [70].

\section{Modified Guildford Model}

The MGM channel has been used in this dissertation to model a rooftop reception scenario, more realistic than i.i.d. Rice. It is based on the model proposed in [70] and extracted from a measurement campaign carried in Guildford (United Kingdom) [122]. The MGM channel is also made up of 8 taps, with different delays and power gains. In this case, each tap also presents a different cross-polarization discrimination value $X$. As occurred with the DVB-NGH channel model, the first tap is Rice distributed with $K$ factor, and the rest are Rayleigh distributed.

The signal model in the time domain is exactly the same as for the DVBNGH mobile channel. It is expressed in the matrix form as in Eq. A.6, and can be decomposed in LoS and NLoS components as in Eq. A.6. The particular values of the power delay profile, as well as the $X$ factor to apply for each tap are shown in Table A.2. The spatial correlation matrix for taps $n=1, \ldots, 8$ is presented below.

$$
\mathbf{R}_{n}=\left[\begin{array}{cccc}
1 & \sqrt{X_{n}} / 8 & 0 & 0.15 \\
\sqrt{X_{n}} / 8 & X_{n} & 0 & 0 \\
0 & 0 & X_{n} & \sqrt{X_{n}} / 8 \\
0.15 & 0 & \sqrt{X_{n}} / 8 & 1
\end{array}\right]
$$

The MGM is characterized by a main LoS component that models low $X$ values with overall value 0.03 . The overall $K$ factor is 5 . Note that this model additionally allows to include a $5.5 \mathrm{~dB}$ standard deviation log-normal distributed fading contribution into the LoS component that represents various network locations. However, the use of this log-normal contribution is not included in this dissertation. 


\section{Appendix B}

\section{Optimization Algorithm}

This appendix presents the algorithm used in this thesis to obtain the optimum values (constellation symbols, rotation angles or quantization reproducers), that provide the maximum BICM capacity [89] for the system model considered. It is based on the Nelder-Mead simplex method, which is one of the most widely used methods for non-linear unconstrained optimization. Although it is widely explained in [101], this appendix provides first the main statement of the algorithm. Afterwards, an example of application is provided, in which the algorithm is adapted to one of the uses cases considered, i.e. constellation optimization.

\section{B.1 Nelder-Mead Simplex Method}

The Nelder-Mead simplex method is a direct search method that endeavors to minimize a non-linear scalar function of $n$ real variables using function values and not using any type of derivative information [123]. This method, among many others, calculates a non-degenerate simplex at each step, i.e. a geometric figure in $n$ dimensions that has $n+1$ vertices. For each iteration, a simplex with $n+1$ vertices is associated to $n+1$ function values. Note that each vertex is directly associated to a particular combination of input parameters (inserted as a vector). One or more vertices are computed with the function values associated, and the iteration ends with bounded level sets.

The algorithm minimizes a real-valued function $f(\mathbf{x})$ for $\mathbf{x} \in \mathcal{R}^{n}$, being $n$ the number of variables to optimize, and $x$ the input parameters. Four scalar parameters are initially defined, which are directly related to the different operations or stages in which the algorithm is divided: $\rho$ (reflection), $\chi$ (expansion), 


\section{APPENDIX B. OPTIMIZATION ALGORITHM}

$\gamma$ (contraction) and $\sigma$ (shrinkage) [101]. The original algorithm proposed in [89] states that the mentioned parameters must satisfy:

$$
\rho>0, \quad \chi>1, \quad \chi>\rho, \quad 0<\gamma<1,0<\sigma<1
$$

The considered expressions in Eq. B.1 comprehend a wide range of possibilities that usually is reduced by selecting concrete values for each one of the variables. The most common choices that satisfy Eq. B.1 are the following:

$$
\rho=1, \quad \chi=2, \gamma=0.5, \sigma=0.5
$$

From now on, values considered in B.2 are assumed. At the beginning of the $k$ th iteration, where $k>0$ and $K$ as the total number of iterations, a new simplex $\Delta_{k}$ is defined, along with its $n+1$ vertices that in turn are points in $\mathcal{R}^{n}$. Prior to the algorithm itself, an initial simplex $\Delta_{0}$ is calculated, which has associated the $n+1$ functions necessary to start with the first stage. An additional parameter $\delta=0.05$ is defined. The calculation is as follows:

- $\mathbf{x}_{1}^{(0)}=\left[x_{11}, x_{12}, \ldots, x_{1 n}\right]$ is taken as the input vector.

- $\mathbf{x}_{2}^{(0)}=\left[(1+\delta) x_{21}, x_{22}, \ldots, x_{2 n}\right]$.

- $\mathbf{x}_{3}^{(0)}=\left[x_{31},(1+\delta) x_{32}, \ldots, x_{3 n}\right]$.

- $\mathbf{x}_{n+1}^{(0)}=\left[x_{(n+1) 1}, x_{(n+1) 2}, \ldots,(1+\delta) x_{(n+1) n}\right]$.

The corresponding functions $f\left(\mathbf{x}^{(0)}\right)$ are then calculated, and the algorithm begins. One iteration of the Nelder-Mead algorithm is divided in the following five stages:

\section{Ordering}

The iteration begins by ordering the vertices as $\mathbf{x}_{1}^{(k)}, \ldots, \mathbf{x}_{n+1}^{(k)}$ so the next expression is satisfied:

$$
f_{1}^{(k)} \leq f_{2}^{(k)} \leq \ldots \leq f_{n+1}^{(k)}
$$

where $f_{i}^{(k)}$ denotes $f\left(\mathbf{x}_{1}^{(k)}\right)$. Logically, since the algorithm tries to minimize $f_{i}^{(k)}$, vertices are ordered such as $\mathbf{x}_{1}^{(k)}$ is the best vertex that provides the lowest value $f_{1}^{(k)}$. Following the same reasoning, $\mathbf{x}_{n+1}^{(k)}$ is taken as the worst vertex, with $f_{n+1}^{(k)}$ as the maximum non-desired value. From now on, the index $k$ is omitted for simplicity. 


\section{Reflection}

The reflection point $\mathbf{x}_{r}$ is computed as follows:

$$
\mathbf{x}_{r}=\overline{\mathbf{x}}+\rho\left(\overline{\mathbf{x}}-\mathbf{x}_{n+1}\right)=(1+\rho) \overline{\mathbf{x}}-\rho \mathbf{x}_{n+1}=2 \overline{\mathbf{x}}-\mathbf{x}_{n+1}
$$

where $\overline{\mathbf{x}}=\sum_{i=1}^{n} \mathbf{x}_{i} / n$ is the centroid of the best $n$ vertices (all except the worst). Thus, the associated function $f_{r}=f\left(\mathbf{x}_{r}\right)$ is calculated, and if $f_{1}<$ $f_{r}<f_{n}$, the reflected vertex is selected and the iteration ends. In the following iteration, $\mathbf{x}_{n+1}$ will be replaced by $\mathbf{x}_{r}$, and the process is repeated again.

\section{Expansion}

If $f_{r}<f_{1}$, the expansion vertex is calculated as:

$$
\begin{aligned}
\mathbf{x}_{e} & =\overline{\mathbf{x}}+\chi\left(\mathbf{x}_{r}-\overline{\mathbf{x}}\right)=\overline{\mathbf{x}}+\rho \chi\left(\overline{\mathbf{x}}-\mathbf{x}_{n+1}\right) \\
& =(1+\rho \chi) \overline{\mathbf{x}}-\rho \chi \mathbf{x}_{n+1}=3 \overline{\mathbf{x}}-2 \mathbf{x}_{n+1}
\end{aligned}
$$

The associated function $f_{e}=f\left(\mathbf{x}_{e}\right)$ is then calculated. If $f_{e}<f_{r}$, the expanded vertex $\mathbf{x}_{e}$ is selected and the iteration ends. Otherwise, if $f_{e} \geq f_{r}$, the vertex selected is $\mathbf{x}_{r}$, and the iteration ends.

\section{Contraction}

If $f_{r} \geq f_{n}$, two different contractions can be performed, depending on $f_{n+1}$.

- Outside contraction: if $f_{n} \leq f_{r} \leq f_{n+1}$, then calculate:

$$
\begin{aligned}
\mathbf{x}_{o c} & =\overline{\mathbf{x}}+\gamma\left(\mathbf{x}_{r}-\overline{\mathbf{x}}\right)=\overline{\mathbf{x}}+\rho \gamma\left(\overline{\mathbf{x}}-\mathbf{x}_{n+1}\right) \\
& =(1+\rho \gamma) \overline{\mathbf{x}}-\rho \gamma \mathbf{x}_{n+1}=1.5 \overline{\mathbf{x}}-0.5 \mathbf{x}_{n+1}
\end{aligned}
$$

The associated function $f_{o c}=f\left(\mathbf{x}_{o c}\right)$ is calculated. If $f_{o c}<f_{r}$, the expanded vertex $\mathbf{x}_{o c}$ is selected and the iteration ends. Otherwise, go to stage 5 .

- Inside contraction: if $f_{r} \geq f_{n+1}$, then calculate:

$$
\mathbf{x}_{i c}=\overline{\mathbf{x}}-\gamma\left(\overline{\mathbf{x}}-\mathbf{x}_{n+1}\right)=(1-\rho) \overline{\mathbf{x}}+\gamma \mathbf{x}_{n+1}=0.5 \mathbf{x}_{n+1}
$$

The associated function $f_{i c}=f\left(\mathbf{x}_{i c}\right)$ is calculated. If $f_{i c}<f_{n+1}$, the expanded vertex $\mathbf{x}_{i c}$ is selected and the iteration ends. Otherwise, go to stage 5 . 


\section{APPENDIX B. OPTIMIZATION ALGORITHM}

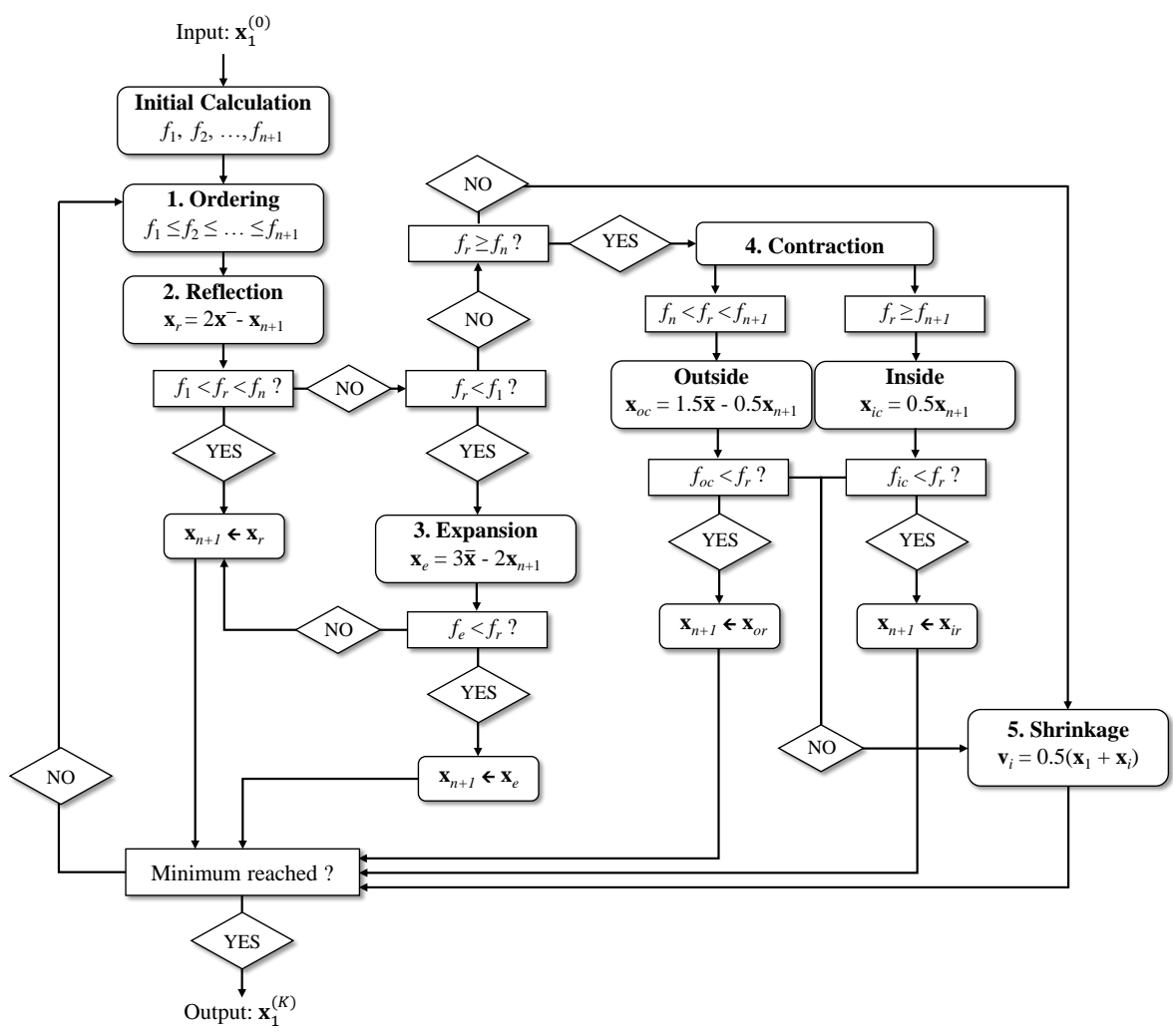

Figure B.1: Flow diagram of the Nelder-Mead optimization algorithm.

\section{Shrinkage}

When none of the criteria considered above is fulfilled, a shrink step is performed. The function $f$ is evaluated at the $n$ vertices as follows:

$$
\mathbf{v}_{i}=\mathbf{x}_{1}+\sigma\left(\mathbf{x}_{i}-\mathbf{x}_{1}\right)=(1-\sigma) \mathbf{x}_{1}+\sigma \mathbf{x}_{i}=0.5\left(\mathbf{x}_{1}+\mathbf{x}_{i}\right)
$$

where $i=2, \ldots, n+1$. The unordered vertices of the simplex for the next iteration is $\mathbf{x}_{1}, \mathbf{v}_{2}, \ldots, \mathbf{v}_{n+1}$.

Fig. B.1 shows the complete flow diagram of the Nelder-Mead algorithm to better understand the concepts explained below. It includes the five stages considered and the possible situations that one can find during the optimization. 


\section{B.2 Application to Use Cases Considered}

In this section, the Nelder-Mead simplex method is applied to one of the use cases, non-uniform constellations optimization. The input vector to the algorithm and the function to calculate for each vector considered in the different iterations are the following:

- Input vector $\mathbf{x}_{1}^{(0)}=\mathbf{a}=\left[a_{1}, a_{2}, \ldots, a_{N}\right]$, being $\mathbf{a}$ the symbol positions to optimize, as explained in Chapter 3.

- Function $f\left(\mathbf{x}_{n}^{(k)}\right)=-C_{B I C M}$, following formula 2.19, defined in Chapter 2.

Since the algorithm seeks for the minimum value of the function defined, the BICM capacity is first calculated and the sign is changed afterwards. This method calculates the negative BICM capacity for each vector $\mathbf{x}_{n}^{(k)}$ considered, and the vector $\mathbf{x}_{1}^{(K)}$ in the last iteration $K$ is finally selected, as shown in Fig. B.1.

As an example, the optimization process for a 1D-64NUC with 3 different parameters, i.e. $\mathbf{a}=\left[a_{1}, a_{2}, a_{3}\right]$, and a $\mathrm{SNR}=20 \mathrm{~dB}$ is detailed step by step. For the case under study, an initial vector with QAM values is chosen, since the SNR selected is high and therefore, parameters will merge toward similar values. Note that indexes for the iteration $k$ are omitted for simplicity.

$k=\mathbf{0}$

Using the parameter $\delta=0.05$, an initial simplex with $n+1=4$ vectors is calculated as follows:

$$
\begin{gathered}
\mathbf{x}=\left[\begin{array}{ccc}
3 & 5 & 7 \\
(1+\delta) 3 & 5 & 7 \\
3 & (1+\delta) 5 & 7 \\
3 & 5 & (1+\delta) 7
\end{array}\right]=\left[\begin{array}{ccc}
3 & 5 & 7 \\
3.15 & 5 & 7 \\
3 & 5.25 & 7 \\
3 & 5 & 7.35
\end{array}\right] \\
f(\mathbf{x})=\left[\begin{array}{l}
-5.814 \\
-5.800 \\
-5.776 \\
-5.822
\end{array}\right]
\end{gathered}
$$


$k=1$

1. Ordering

$$
\mathbf{x}=\left[\begin{array}{ccc}
3 & 5 & 7.35 \\
3 & 5 & 7 \\
3.15 & 5 & 7 \\
3 & 5.25 & 7
\end{array}\right], f(\mathbf{x})=\left[\begin{array}{l}
-5.822 \\
-5.814 \\
-5.800 \\
-5.776
\end{array}\right]
$$

2. Reflection

$$
\overline{\mathbf{x}}=\left[\begin{array}{lll}
3.05 & 5 & 7.116
\end{array}\right]
$$

$$
\mathbf{x}_{r}=1.5 \overline{\mathbf{x}}-0.5 \mathbf{x}_{n+1}=\left[\begin{array}{lll}
3.1 & 4.75 & 7.233
\end{array}\right], f\left(\mathbf{x}_{r}\right)=-5.784
$$

- $f_{1}<f_{r}<f_{n} ? \rightarrow$ No.

- $f_{r}<f_{1} ? \rightarrow$ No.

- $f_{r} \geq f_{n}$ ? $\rightarrow$ Yes: Contraction.

- $f_{n}<f_{r}<f_{n+1}$ ? $\rightarrow$ Yes: Outside Contraction.

4. Outside Contraction

$$
\mathbf{x}_{o c}=1.5 \overline{\mathbf{x}}-0.5 \mathbf{x}_{n+1}=\left[\begin{array}{lll}
3.07 & 4.87 & 7.175
\end{array}\right], f\left(\mathbf{x}_{o c}\right)=-5.809
$$

- $f_{o c}<f_{r} ? \rightarrow$ Yes: select $\mathbf{x}_{o c}$

$$
\mathbf{x}=\left[\begin{array}{ccc}
3 & 5 & 7.35 \\
3 & 5 & 7 \\
3.15 & 5 & 7 \\
3.07 & 4.87 & 7.175
\end{array}\right], f(\mathbf{x})=\left[\begin{array}{l}
-5.822 \\
-5.814 \\
-5.800 \\
-5.809
\end{array}\right]
$$

$k=2$

1. Ordering

$$
\mathbf{x}=\left[\begin{array}{ccc}
3 & 5 & 7.35 \\
3 & 5 & 7 \\
3.07 & 4.87 & 7.175 \\
3.15 & 5 & 7
\end{array}\right], f(\mathbf{x})=\left[\begin{array}{l}
-5.822 \\
-5.814 \\
-5.809 \\
-5.800
\end{array}\right]
$$


2. Reflection

$$
\begin{gathered}
\overline{\mathbf{x}}=\left[\begin{array}{lll}
3.025 & 4.958 & 7.175
\end{array}\right] \\
\mathbf{x}_{r}=1.5 \overline{\mathbf{x}}-0.5 \mathbf{x}_{n+1}=\left[\begin{array}{lll}
2.9 & 4.916 & 7.35
\end{array}\right], f\left(\mathbf{x}_{r}\right)=-5.819
\end{gathered}
$$

- $f_{1}<f_{r}<f_{n}$ ? $\rightarrow$ Yes: select $\mathbf{x}_{r}$.

$$
\mathbf{x}=\left[\begin{array}{ccc}
3 & 5 & 7.35 \\
3 & 5 & 7 \\
3.07 & 4.87 & 7.175 \\
2.9 & 4.916 & 7.35
\end{array}\right], f(\mathbf{x})=\left[\begin{array}{l}
-5.822 \\
-5.814 \\
-5.809 \\
-5.819
\end{array}\right]
$$

Although for $k=1$ and $k=2$ only three different stages are used, the rest are usually considered in further iterations. For instance, in this case an expansion is performed when $k=23$ as follows:

$k=23$

1. Ordering

$$
\mathbf{x}=\left[\begin{array}{ccc}
2.944 & 4.992 & 7.279 \\
2.945 & 4.992 & 7.277 \\
2.943 & 4.991 & 7.281 \\
2.945 & 4.99 & 7.28
\end{array}\right], f(\mathbf{x})=\left[\begin{array}{l}
-5.8237 \\
-5.8236 \\
-5.8234 \\
-5.8233
\end{array}\right]
$$

2. Reflection

$$
\begin{aligned}
& \overline{\mathbf{x}}=\left[\begin{array}{lll}
2.944 & 4.991 & 7.279
\end{array}\right] \\
& \mathbf{x}_{r}=1.5 \overline{\mathbf{x}}-0.5 \mathbf{x}_{n+1}=\left[\begin{array}{lll}
2.943 & 4.991 & 7.276
\end{array}\right], f\left(\mathbf{x}_{r}\right)=-5.8242 \\
& \text { - } f_{1}<f_{r}<f_{n} ? \rightarrow \text { No. } \\
& \text { - } f_{r}<f_{1} ? \rightarrow \text { Yes: Expansion. }
\end{aligned}
$$

3. Expansion

$$
\mathbf{x}_{e}=3 \overline{\mathbf{x}}-2 \mathbf{x}_{n+1}=\left[\begin{array}{lll}
2.942 & 4.99 & 7.275
\end{array}\right], f\left(\mathbf{x}_{e}\right)=-5.8237
$$


- $f_{r}<f_{1} ? \rightarrow$ No: select $\mathbf{x}_{r}$.

$$
\mathbf{x}=\left[\begin{array}{lll}
2.944 & 4.992 & 7.279 \\
2.945 & 4.992 & 7.277 \\
2.943 & 4.991 & 7.281 \\
2.943 & 4.991 & 7.276
\end{array}\right], f(\mathbf{x})=\left[\begin{array}{l}
-5.8237 \\
-5.8236 \\
-5.8234 \\
-5.8242
\end{array}\right]
$$

Note that all possible $\mathbf{x}$ are very similar amongst each other for this $k$ value, since the algorithm smoothly approaches the optimum $\mathbf{x}$ that gives the maximum BICM capacity (looking for the the minimum negative BICM capacity). 


\section{Acronyms}

ATSC Advanced Television Systems Committee

ATSC 3.0 ATSC - Third Generation

AWGN Additive White Gaussian Noise

BCH Bose Chadhuri Hocquenghem

BER Bit Error Rate

BICM Bit-Interleaved Coded Modulation

BICM-ID Iteratively Decoded Bit-Interleaved Coded Modulation

BIL Bit Interleaver

CB Channel Bonding

CR Coding Rates

CIL Component Interleaver

CM Coded Modulation

CSI Channel State Information

CSR Condensed Symbols Reduction

DD Digital Dividend

DD2 Second Digital Dividend

DL Downlink

DoF Degrees of Freedom 
DT Decision Threshold

DTMB Digital Terrestrial Multimedia Broadcast

DTT Digital Terrestrial Television

DVB-NGH Digital Video Broadcasting - Next Generation Handheld

DVB-T Digital Video Broadcasting - Terrestrial

DVB-T2 DVB - Terrestrial Second Generation

FBMC Filter Bank Multi-Carrier

FDM Frequency Division Multiplexing

FEC Forward Error Correction

FFT Fast Fourier Transform

FQAM Frequency and Quadrature Amplitude Modulation

FSD Fixed-Complexity Sphere Decoders

FSK Frequency Shift Keying

GI Guard Intervals

HDTV High Definition TV

HDR High Dynamic Range

i.i.d. independent and identically distributed

IMT International Mobile Telecommunications

ISDB-T Integrated Services Digital Broadcasting - Terrestrial

ISI Inter-Symbol Interference

ITU International Telecommunications Union

LDM Layered Division Multiplexing

LDPC Low Density Parity Check

LLR Log-Likelihood Ratios

LoS Line-of-Sight 
LSB Least Significant Bits

LTE Long Term Evolution

LUT Look-Up Table

MA-NUC Multi-Antenna Non-Uniform Constellation

MGM Modified Guildford Model

MIMO Multiple-Input Multiple-Output

MISO Multiple-Input Single-Output

ML Maximum Likelihood

MMSE Minimum Mean Square Error

MSB Most Significant Bits

NLoS Non-Line-of-Sight

NOMA Non-Orthogonal Multiple Access

NUC Non-Uniform Constellation

NURC Non-Uniform Rotated Constellation

OAR Optimization with Additional Rotation

OBR Optimization Before Rotation

OFDM Orthogonal Frequency-Division Multiplexing

OQAM Offset Quadrature Amplitude Modulation

PHY Physical Layer

PI Power Imbalance

pdf probability density function

PLP Physical Layer Pipes

PP Pilot Pattern

p-t-p point-to-point

QAM Quadrature Amplitude Modulation 
QCSR Quadrant Condensed Search Reduction

QSR Quadrant Search Reduction

RC Rotated Constellations

RF Radio Frequency

SD Sphere Decoder

SDTV Standard Definition TV

SFSD Soft Fixed-Complexity Sphere Decoders

SIC Successive Interference Cancellation

SIMO Single-Input Multiple-Output

SM Spatial Multiplexing

SNR Signal-to-Noise Ratio

SSD Signal Space Diversity

TDIL Time De-Interleaver

TDM Time Division Multiplexing

TFS Time-Frequency Slicing

TIL Time Interleaver

TV Television

UHDTV Ultra High-Definition TV

UL Uplink

UHF Ultra-High Frequency

WRC World Radiocommunication Conference

XPD Cross-Polar Discrimination

ZF Zero Forcing

3GPP Third Generation Partnership Project 


\section{References}

[1] R. Beutler, Digital Terrestrial Broadcasting Networks. Lecture Notes in Electrical Engineering (LNEE). Springer, 2009, vol. 23.

[2] "Roadmap for the Evolution of DTT - A bright Future for TV," Digital Television Action Group (DIGITAG), Tech. Rep., 2015.

[3] W. Sami, "How can Mobile and Broadcasting Networks use Adjacent Bands?" European Broadcasting Union (EBU), Digital Dividend Technical Report, 2011.

[4] M. Fuentes et al., "Coexistence of Digital Terrestrial Television and Next Generation Cellular Networks in the $700 \mathrm{MHz}$ Band," IEEE Wireless Communications, vol. 21, no. 6, pp. 63-69, Dec. 2014.

[5] D. Gomez-Barquero, and M. W. Caldwell, "Broadcast Television Spectrum Incentive Auctions in the U.S.: Trends, Challenges, and Opportunities," IEEE Commun. Magazine, vol. 53, no. 7, pp. 50-56, July 2015.

[6] G. A. Reitmeier and T. R. Smith, "An Overview of the ATSC Digital Television Standard," in International Workshop on HDTV, Los Angeles, USA, Oct. 1996.

[7] M. Takada, and M. Saito, "Transmission System for ISDB-T," Proceedings of the IEEE, vol. 94, no. 1, pp. 251-256, Jan. 2006.

[8] J. Song et al., "Technical Review on Chinese Digital Terrestrial Television Broadcasting Standard and Measurements on Some Working Modes," IEEE Transactions on Broadcasting, vol. 53, no. 1, pp. 1-7, March 2007.

[9] U. Ladebusch and C. A. Liss, "Terrestrial DVB (DVB-T): A Broadcast Technology for Stationary Portable and Mobile Use," Procedings of the IEEE, vol. 94, no. 1, pp. 183-193, Jan. 2006. 
[10] W. Y. Zou and Y. Wu, "COFDM: an Overview," IEEE Transactions on Broadcasting, vol. 41, no. 1, pp. 1-8, March 1995.

[11] C. E. Shannon, "A Mathematical Theory of Communication," Bell System Technical Journal, vol. 27, pp. 379-423, 623-656, 1948.

[12] I. Eizmendi et al., "DVB-T2: The Second Generationof Terrestrial Digital Video Broadcasting System," IEEE Transactions on Broadcasting, vol. 60 , no. 2, pp. 258-271, June 2014.

[13] D. Gomez-Barquero, "Cost Efficient Provisioning of Mass Mobile Multimedia Services in Hybrid Cellular and Broadcasting Systems," Ph.D. dissertation, Universitat Politecnica de Valencia, Valencia, Spain, 2009.

[14] D. Gomez-Barquero, C. Douillard, P. Moss, and V. Mignone, "DVBNGH: The Next Generation of Digital Broadcast Services to Handheld Devices," IEEE Transactions on Broadcasting, vol. 60, no. 2, pp. 246-257, June 2014.

[15] L. Fay et al., "An Overview of the ATSC 3.0 Physical Layer Specification," IEEE Transactions on Broadcasting, vol. 62, no. 1, pp. 159-171, March 2016.

[16] S. I. Park et al., "Low Complexity Layered Division Multiplexing for ATSC 3.0," IEEE Transactions on Broadcasting, vol. 62, no. 1, pp. 233243, March 2016.

[17] N. Loghin et al., "Non-Uniform Constellations for ATSC 3.0," IEEE Transactions on Broadcasting, vol. 62, no. 1, pp. 197-203, March 2016.

[18] A. Alvarado, E. Agrell, and A. Svensson, On the capacity of BICM with QAM constellations. Chalmers Publication Library, 2009.

[19] J. Massey, "Coding and Modulation in Digital Communications," in International Zürich Seminar on Digital Communications, Zurich, Switzerland, March 1974.

[20] G. Ungerboeck, "Channel Coding with Multilevel/Phase Signals," IEEE Trans. Inf. Theory, vol. 28, no. 1, pp. 55-67, Jan. 1982.

[21] H. Imai, and S. Hirakawa, "A New Multilevel Coding Method using Error Correcting Codes," IEEE Trans. Inf. Theory, vol. 23, no. 3, pp. 371-377, May 1977. 
[22] G. Caire, G. Taricco, and E. Biglieri, "Bit-Interleaved Coded Modulation," IEEE Trans. Inform. Theory, vol. 44, no. 3, pp. 927-946, May 1998.

[23] A. Guillen i Fabregas, A. Martinez, and G. Caire, "Bit-Interleaved Coded Modulation," Found. Trends Commun. Inf. Theory, vol. 5, no. 1-2, pp. 1-153, Nov. 2008.

[24] J. Zöllner, and N. Loghin, "Optimization of High-Order Non-Uniform QAM Constellations," in Proc. IEEE Int. Symp. Broadband Multimedia Syst. Broadcast. (BMSB), London, UK, June 2013.

[25] D. Gozalvez, J. J. Gimenez, D. Gomez-Barquero, and N. Cardona, "Rotated Constellations for Improved Time and Frequency Diversity in DVBNGH," IEEE Transactions on Broadcasting, vol. 59, no. 2, pp. 298-305, June 2013.

[26] P. Xue, K. Bae, K. Kim, and H.Yang, "Best Candidates Based SoftDemapper for Rotated M-QAM Constellation," in IEEE 11th Consumer Communications and Networking Conference $(C C N C)$, Las Vegas, USA, Jan. 2014.

[27] D. Vargas, "Transmit and Receive Signal Processing for MIMO Terrestrial Broadcast Systems," Ph.D. dissertation, Universitat Politecnica de Valencia, Valencia, Spain, May 2016.

[28] "Digital Video Broadcasting (DVB); Implementation guidelines for a second generation digital terrestrial television broadcasting system (DVBT2)," ETSI TS 102831 V1.2.1, Aug. 2012.

[29] A. Goldsmith, S. A. Jafar, N. Jindal, and S. Vishwanath, "Capacity Limits of MIMO channels," IEEE J. Sel. Areas Commun., vol. 21, no. 5, pp. 684-702, June 2003.

[30] D. Tse and P. Viswanath, Fundamentals of Wireless Communications. Cambridge University Press, 2015.

[31] E. Zehavi, "8-PSK Trellis Codes for a Rayleigh Channel," IEEE Trans. Communications, vol. 40, no. 3, pp. 873-884, May 1992.

[32] A. Alvarado, "On Bit-interleaved Coded Modulation with QAM Constellations," Ph.D. dissertation, Chalmers University of Technology, Göteborg, Sweden, 2008. 
[33] D. Gomez-Barquero, Next Generation Mobile Broadcasting. Ed. Boca Raton, FL, USA: CRC Press, 2013.

[34] L. Michael, and D. Gomez-Barquero, "Bit-interleaved Coding and Modulation (BICM) for ATSC 3.0," IEEE Trans. Broadcast., vol. 62, no. 1, pp. 181-188, March 2016.

[35] K. J. Kim et al., "Low-Density Parity-Check Codes for ATSC 3.0," IEEE Trans. Broadcast., vol. 62, no. 1, pp. 189-196, March 2016.

[36] G. J. Foschini, R. Gitlin, and S. Weinstein, "Optimization of TwoDimensional Signal-Constellations in the Presence of Gaussian Noise," IEEE Trans. Commun., vol. 22, no. 1, pp. 28-38, Jan. 1974.

[37] J. Stott, "BICM Limits for Condensed QAM, Further Results," DVB document TM-MIMO0023, Feb. 2013.

[38] J. Stott, "CM and BICM Limits for Rectangular Constellations," White Paper 257, BBC Research and Development, Aug. 2013.

[39] B. Mouhouche, D. Ansorregui, and A. Mourad, "High Order NonUniform Constellations for Broadcasting UHDTV," in Proc. IEEE Wireless Commun. Netw. Conf. (WCNC), Istambul, Turkey, April 2014.

[40] J. Morgade et al., "Improving the DVB-T2 BICM Performance by newly Optimized Two-Dimensional Non-Uniform Constellations," in IEEE Fourth International Conference on Consumer Electronics (ICCE), Berlin, Germany, Sept. 2014.

[41] T. Arafa, W. Sauer-Greff, and R. Urbansky, "Non-Uniform Signal Constellation for Iteratively Decoded Bit Interleaved Coded Modulation (BICM-ID) with Convolution and LDPC Codes," in International Conference on Innovations in Information Technology (IIT), Abu Dhabi, UAE, April 2011.

[42] C. Barjau et al., "Improvement of L1 Signaling Performance for DVBT2 Using Optimized Two-Dimensional Non-Uniform Constellations," in IEEE International Conference on Ubiquitous Wireless Broadband (ICUWB), Montreal, Canada, Oct. 2015.

[43] J. Stott, "Beyond NUQAM \& ConQAM - Overcoming their Limitations, Especially at Lower SNRs," DVB document TM-T0007, Jan. 2014.

[44] S. Kwon et al., "Simplified Non-Uniform Constellation Demapping Scheme for the Next Broadcasting System," in IEEE Int. Symp. Broadband Multimedia Syst. Broadcast. (BMSB), Ghent, Belgium, June 2015. 
[45] J. Barrueco et al., "Low Complexity and High Order Two-Dimensional Non-Uniform Constellations for High Capacity Broadcasting Systems," in IEEE International Symposium on Broadband Multimedia Systems and Broadcasting (BMSB), Nara, Japan, July 2016.

[46] B. Mouhouche, C. Barjau, and H. Lee, "Design of Non Uniform Constellations for Layered Division Multiplexing," in IEEE International Symposium on Signal Processing and Information Technology (ISSPIT), Abu Dhabi, UAE, Sept. 2015.

[47] J. Montalban, "Solutions for New Terrestrial Broadcasting Systems Offering Simultaneously Stationary and Mobile Services," Ph.D. dissertation, University of the Basque Country, Bilbao, Spain, Dec. 2014.

[48] D. Gomez-Barquero, and O. Simeone, "LDM vs. FDM/TDM for Unequal Error Protection in Terrestrial Broadcasting Systems: An InformationTheoretic View," IEEE Trans. Broadcast., vol. 61, no. 4, pp. 571-579, Aug. 2015.

[49] J. Boutros and E. Viterbo, "Signal Space Diversity: A Power- and Bandwidth-Efficient Diversity Technique for the Rayleigh Fading Channel," IEEE Trans. Inform. Theory, vol. 44, no. 4, pp. 1453-1467, July 1998.

[50] Q. Xie et al., "Coded Modulation with Signal Space Diversity," IEEE Trans. on Wireless Communications, vol. 10, no. 2, pp. 660-669, Dec. 2010.

[51] C. A. Nour, and C. Douillard, "Improving BICM Performance of QAM Constellations for Broadcasting Applications," in 5th International Symposium on Turbo Codes and Related Topics, Lausanne, Switzerland, Sept. 2008.

[52] D. Gozalvez, "Combined Time, Frequency and Space Diversity in Multimedia Mobile Broadcasting Systems," Ph.D. dissertation, Universitat Politecnica de Valencia, Valencia, Spain, June 2012.

[53] D. Perez-Calderon et al., "Rotated Constellation Demapper for DVBT2," IEEE Electron. Lett., vol. 47, no. 1, pp. 31-32, Jan. 2011.

[54] D. Perez-Calderon et al., "Simplified Rotated Constellation Demapper for Second Generation Terrestrial Digital Video Broadcasting," IEEE Trans. Broadcast., vol. 59, no. 1, pp. 160-167, March 2013. 
[55] S. Tomasin, and M. Butussi, "Low Complexity Demapping of Rotated and Cyclic Q Delayed Constellations for DVB-T2," IEEE Wireless Communications Letters, vol. 1, no. 2, pp. 81-84, April 2012.

[56] B. Hassibi, and H. Vikalo, "On the Sphere-Decoding Algorithm I. Expected Complexity," IEEE Tans. Signal Processing, vol. 53, no. 8, pp. 2806-2818, Aug. 2005.

[57] J. Yang et al., "Max-Log Demapper Architecture Design for DVB-T2 Rotated QAM Constellations," in IEEE Workshop on Signal Processing Systems (SiPS), Dallas, USA, Oct. 2015.

[58] D. Gozalvez, D. Gomez-Barquero, D. Vargas, and N. Cardona, "Combined Time, Frequency and Space Diversity in DVB-NGH," IEEE Trans. Broadcast., vol. 59, no. 4, pp. 674-684, Dec. 2013.

[59] M. Makni, J. Robert, and E. Stare, "Performance Analysis of Time Frequency Slicing," in 14th ITG Conference on Electronic Media Technology (CEMT), Dortmund, Germany, March 2011.

[60] J. J. Gimenez, E. Stare, S. Bergsmark, and D. Gomez-Barquero, "Time Frequency Slicing for Future Digital Terrestrial Broadcasting Networks," IEEE Trans. Broadcast., vol. 60, no. 2, pp. 227-238, June 2014.

[61] J. J. Gimenez, "Improved Spectrum Usage with Multi-RF Channel Aggregation Technologies for the Next-Generation Terrestrial Broadcasting," Ph.D. dissertation, Universitat Politecnica de Valencia, Valencia, Spain, June 2015.

[62] L. Stadelmeier, D. Schneider, J. Zöllner, and J. J. Gimenez, "Channel bonding for ATSC 3.0," IEEE Trans. Broadcast., vol. 62, no. 1, pp. 289297, March 2016.

[63] E. Garro, J. J. Gimenez, S. Ik Park, and D. Gomez-Barquero, "Layered Division Multiplexing With Multi-Radio-Frequency Channel Technologies," IEEE Trans. Broadcast., vol. 62, no. 2, pp. 365-374, June 2016.

[64] E. Stare, J. J. Gimenez, and P. Klenner, "WIB $\ddot{i} ; \frac{1}{2}$ A New System Concept for Digital Terrestrial Television (DTT)," in International Broadcasting Convention (IBC), Amsterdam, Netherlands, Sept. 2016.

[65] J. Max, "Quantizing for Minimum Distortion," IEEE Trans. Information Theory, vol. 6, no. 1, pp. 7-12, March 1960. 
[66] A. Gersho and R. M. Gray, Vector Quantization and Signal Compression. Kluwer Academic Publishers, 1992.

[67] R. M. Gray and D. L. Neuhoff, "Quantization," IEEE Trans. Information Theory, vol. 44, no. 6, pp. 2325-2383, Oct. 1998.

[68] A. Winkelbauer, and G. Matz, "On Quantization of Log-Likelihood Ratios for Maximum Mutual Information," in IEEE International Workshop on Signal Processing Advances in Wireless Communications (SPAWC), Stockholm, Sweeden, June 2015.

[69] S. Rosati, S. Tomasin, M. Butussi, and B. Rimoldi, "LLR Compression for BICM Systems Using Large Constellations," IEEE Trans. Communications, vol. 61, no. 7, pp. 2864-2875, July 2013.

[70] P. Moss, "2-by-2 MIMO Fixed Reception Channel Model for Dual-Polar Terrestrial Transmission," White Paper 161, BBC Research and Development, Jan. 2008.

[71] D. Vargas et al., "A MIMO-Channel-Precoding Scheme for Next Generation Terrestrial Broadcast TV Systems," IEEE Trans. Broadcast., vol. 61, no. 3 , pp. 445-456, Sept. 2015.

[72] J. Jang, D. H. Kim, and Y. H. Lee, "Open-Loop Precoder for Spatial Multiplexing in MIMO Channels with Phase Correlation," IET Electronic Letters, vol. 50, no. 23, pp. 1766-1768, Nov. 2014.

[73] S. Saito et al., "8K Terrestrial Transmission Field Tests using Dualpolarized MIMO and Higher-Order Modulation OFDM," IEEE Trans. Broadcast., vol. 62, no. 1, pp. 306-315, Jan. 2016.

[74] P. Fertl, J. Jaldi $; \frac{1}{2} \mathrm{n}$, and G. Matz, "Performance Assessment of MIMOBICM Demodulators Based on Mutual Information," IEEE Trans. Signal Processing, vol. 60, no. 3, pp. 1366-1382, March 2012.

[75] D. Vargas, D. Gozalvez, D. Gomez-Barquero, and N. Cardona, "MIMO for DVB-NGH, the Next Generation Mobile TV Broadcasting," IEEE Commun. Magazine, vol. 51, no. 7, pp. 130-137, July 2013.

[76] D. Gomez-Barquero et al., "MIMO for ATSC 3.0," IEEE Trans. Broadcast., vol. 62, no. 1, pp. 298-305, March 2016.

[77] T. Shitomi et al., "Field Experiments of Dual-Polarized MIMO-OFDM Transmission Using Non-Uniform Constellations," in IEEE International Conference on Consumer Electronics (ICCE), Berlin, Germany, Jan. 2016. 
[78] D. Perez-Calderon et al., "Simplified Detection for DVB-NGH MIMO Decoders," IEEE Trans. Broadcast., vol. 61, no. 1, pp. 84-90, March 2015.

[79] T. Shitomi et al., "Evaluation of Simplified Demapping Algorithm for NUQAMs Using Urban MIMO Channel Response," in IEEE International Symposium on Broadband and Multimedia Systems and Broadcasting (BMSB), Nara, Japan, June 2016.

[80] T. Kailath, H. Vikalo, and B. Hassibi, MIMO Receive Algorithms. Cambridge University Press, 2005.

[81] E. Viterbo and J. Boutros, "A Universal Lattice Decoder for Fading Channels," IEEE Trans. on Information Theory, vol. 45, no. 5, p. $1639 i \mathrm{i} ; \frac{1}{2} 1642$, July 1999 .

[82] S. Roger, "Design and Implementation of Efficient Algorithms for Wireless MIMO Communication Systems," Ph.D. dissertation, Universitat Politecnica de Valencia, Valencia, Spain, July 2012.

[83] L. G. Barbero, T. Ratnarajah, and C. Cowan, "A Low-Complexity SoftMIMO Detector based on the Fixed-Complexity Sphere Decoder," in IEEE International Conference on Acoustics, Speech and Signal Processing, Las Vegas, USA, March 2008.

[84] C. Studer, M. Wenk, A. Burg, and H. Bölcskei, "Soft-Output Sphere Decoding: Performance and Implementation Aspects," in Asilomar Conference on Signals, Systems and Computers (ACSSC), Pacific Grove, USA, Nov. 2006.

[85] H. Nyquist, "Certain Topics in Telegraph Transmission Theory," Reedited manuscript, Proceedings of the IEEE, vol. 90, no. 2, pp. 280-305, Feb. 2002.

[86] E. Telatar, "Capacity of Multi-Antenna Gaussian Channels," European Trans. Telecomm., vol. 10, no. 6, pp. 585-596, Nov. 1999.

[87] G. Strang, Introduction to Linear Algebra. 4th Ed. Wellesley-Cambridge Press, 2009.

[88] C. Barjau, M. Fuentes, T. Shitomi and D. Gomez-Barquero, "MIMO Sphere Decoder with Successive Interference Cancellation for TwoDimensional Non-Uniform Constellations," IEEE Communications Letters, Jan. 2017. 
[89] J. A. Nelder, and R. Mead, "A Simplex Method for Function Minimization," Computer Journal, vol. 7, no. 4, pp. 308-313, 1965.

[90] J. Barry, E. Lee, and D. Messerschmitt, Digital Communications. United States: Ed. Springer, 2003.

[91] D. Seethaler, G. Matz, and F. Hlawatsch, "An effcient MMSE-based Demodulator for MIMO Bit-Interleaved Coded Modulation," in IEEE Globecom, Dallas, USA, Nov. 2004.

[92] C. Douillard et al., "Iterative Correction of Intersymbol Interference: Turbo-Equalisation," European Trans. Telecomm., vol. 6, no. 5, pp. 507511, Sept. 1995.

[93] R. Koetter, A. Singer, and M. Tuchler, "Turbo Equalization," IEEE Signal Proc. Magazine, vol. 21, no. 1, pp. 67-80, Jan. 2004.

[94] M. Witzke, S. Bäro, F. Schreckenbach, and J. Hagenauer, "Iterative Detection of MIMO Signals with Linear Detectors," in Asilomar Conference on Signals, Systems and Computers, Pacific Groove, USA, Nov. 2002.

[95] E. Agrell, T. Eriksson, A. Vardy, and K. Zeger, "Closest Point Search in Lattices," IEEE Trans. on Information Theory, vol. 48, no. 8, pp. 2201-2214, Aug. 2002.

[96] Z. Guo, and P. Nilsson, "Algorithm and Implementation of the K-Best Sphere Decoding for MIMO Detection," IEEE Journal on Selected Areas in Communications, vol. 24, no. 3, pp. 491-503, March 2006.

[97] M. O. Damen, H. E. Gamal, and G. Caire, "On Maximum-Likelihood Detection and the Search for the Closest Lattice Point," IEEE Trans. Inf. Theory, vol. 49, no. 10, pp. 2389-2402, Oct. 2003.

[98] L. G. Barbero, and J. S. Thompson, "Fixing the Complexity of the Sphere Decoder for MIMO Detection," IEEE Trans. Wireless Communications, vol. 7 , no. 6, pp. 2131-2142, June 2008.

[99] J. Jalden, L. G. Barbero, B. Ottersten, and J. S. Thompson, "The Error Probability of the Fixed-Complexity Sphere Decoder," IEEE Trans. on Signal Processing, vol. 57, no. 7, pp. 2711-2720, July 2009.

[100] G. D. Forney Jr., and L.-F. Wei, "Multidimensional Constellations $\dddot{i}_{i} i \frac{1}{2}$ Part I: Introduction, Figures of Merit and Generalized Cross Constellations," IEEE Journal on Selected Areas in Communications, vol. 1, no. 6, pp. 877-892, Aug. 1989. 
[101] J. C. Lagarias, J. A. Reeds, M. H. Wright, and P. E. Wright, "Convergence Properties of the Nelder-Mead Simplex Method in Low Dimensions," SIAM Journal of Optimization, vol. 9, no. 1, pp. 112-147, 1998.

[102] L. Yang, and Z. Huiyan, "Shape Preserving Piecewise Cubic Interpolation," Applied Mathematics, vol. 11, no. 4, pp. 419-424, Dec. 1996.

[103] M. Fuentes, J. J. Gimenez and D. Gomez-Barquero, "Optimization and Performance of Non-Uniform Rotated Constellations with Multi-RF Transmission Techniques," IEEE Trans. Broadcast., vol. 62, no. 4, pp. 855-863, Dec. 2016.

[104] M. Fuentes, D. Vargas and D. Gomez-Barquero, "Low-Complexity Demapping Algorithm for Two-Dimensional Non-Uniform Constellations," IEEE Trans. Broadcast., vol. 62, no. 2, pp. 375-383, June 2016.

[105] J. J. Gimenez, D. Gozalvez, D. Gomez-Barquero, and N. Cardona, "Statistical Model of Signal Strength Imbalance Between RF Channels in DTT Network," Electronic Letters, vol. 48, no. 12, pp. 731-732, June 2012.

[106] T. M. Cover, Elements of Information Theory. John Wiley \& Sons Inc., 2001, pp. 336-373.

[107] A. Winkelbauer, "Blind Performance Estimation and Quantizer Design with Applications to Relay Networks," Ph.D. dissertation, Technische Universtität Wien, Vienna, Austria, Dec. 2014.

[108] J. Liang, L. Thiele, and V. Jungnickel, "On the Modelling of Polarized MIMO Channel," in 13th European Wireless Conference, Paris, France, April 2007.

[109] G. H. Golub, and C. F. Van Loan, Matrix Computations. The Johns Hopkins Univesity Press, 3rd Edition, 1996.

[110] F. Aurenhammer, "Voronoi Diagrams - A Survey of a Fundamental Geometric Data Structure," ACM Computing Surveys, vol. 23, no. 3, pp. 345-405, Sept. 1991.

[111] G. J. Sullivan, J. Ohm, Woo-Jin Han, and T. Wiegand, "Overview of the High Efficiency Video Coding (HEVC) Standard," IEEE Transactions on Circuits and Systems for Video Technology, vol. 22, no. 12, pp. 16491668, Dec. 2012. 
[112] W. Li et al., "Using LDM to achieve seamless local service coverage in SFN environment," in IEEE International Symposium on Broadband Multimedia Systems and Broadcasting (BMSB), Nara, Japan, July 2016.

[113] J. Calabuig, J. F. Monserrat, and D. Gomez-Barquero, "5th Generation Mobile Networks: A new Opportunity for the Convergence of Mobile Broadband and Broadcast Services," IEEE Communications Magazine, vol. 53, no. 2, pp. 198-205, Feb. 2015.

[114] S. Hong et al., "FQAM : A Modulation Scheme for Beyond 4G Cellular Wireless Communication Systems," in IEEE Globecom Workshops (GC Wkshps), Atlanta, USA, June 2013.

[115] V. Vakilian et al., "Universal-Filtered Multi-Carrier Technique for Wireless Systems Beyond LTE," in IEEE Globecom Workshops, Atlanta, USA, Dec. 2013.

[116] E. Larsson, O. Edfors, F. Tufvesson, and T. Marzetta, "Massive MIMO for Next Generation Wireless Systems," IEEE Communications Magazine, vol. 52, no. 2, pp. 186-195, Feb. 2014.

[117] A. Benjebbour et al., "Concept and Practical Considerations of NonOrthogonal Multiple Access (NOMA) for Future Radio Access," in Proc. IEEE ISPACS, Okinawa, Japan, Nov. 2013.

[118] P. Klenner et al., "Physical Layer Time Interleaving for the ATSC 3.0 System," IEEE Trans. Broadcast., vol. 62, no. 1, pp. 253-262, March 2016.

[119] M. Earnshaw et al., "Physical Layer Framing for ATSC 3.0," IEEE Trans. Broadcast., vol. 62, no. 1, pp. 263-27, March 2016.

[120] S. M. Alamouti, "A Simple Transmit Diversity Technique for Wireless Communications," IEEE J. Sel. Areas Comm., vol. 16, no. 10, pp. 14511458, Oct. 1998.

[121] P. Moss, T. Y. Poon, and J. Boyer, "A Simple Model of the UHF Crosspolar Terrestrial Channel for DVB-NGH," White Paper 205, BBC Research and Development, Sept. 2011.

[122] J. Boyer et al., "MIMO for Broadcast-Results from a High-Power UK Trial," White Paper 157, BBC Research and Development, Jan. 2007.

[123] M. Wright, Direct Search Methods: Once Scorned, Now Respectable. Addison-Wesley, Numerical analysis: Proceedings of the 1995 Dundee Biennial Conference in Numerical Analysis, 1996, pp. 191-208. 